\title{
Mitochondrial dysfunction in the diabetic heart: Cardioprotection from a novel mitochondrially-targeted therapeutic
}

\author{
Erinne Rose Dabkowski \\ West Virginia University
}

Follow this and additional works at: https://researchrepository.wvu.edu/etd

\section{Recommended Citation}

Dabkowski, Erinne Rose, "Mitochondrial dysfunction in the diabetic heart: Cardioprotection from a novel mitochondrially-targeted therapeutic" (2010). Graduate Theses, Dissertations, and Problem Reports.

3251.

https://researchrepository.wvu.edu/etd/3251

This Dissertation is protected by copyright and/or related rights. It has been brought to you by the The Research Repository @ WVU with permission from the rights-holder(s). You are free to use this Dissertation in any way that is permitted by the copyright and related rights legislation that applies to your use. For other uses you must obtain permission from the rights-holder(s) directly, unless additional rights are indicated by a Creative Commons license in the record and/ or on the work itself. This Dissertation has been accepted for inclusion in WVU Graduate Theses, Dissertations, and Problem Reports collection by an authorized administrator of The Research Repository @ WVU.

For more information, please contact researchrepository@mail.wvu.edu. 


\title{
Mitochondrial Dysfunction in the Diabetic Heart: Cardioprotection from a Novel Mitochondrially- Targeted Therapeutic
}

\author{
Erinne Rose Dabkowski \\ Dissertation submitted to the School of Medicine \\ at West Virginia University \\ in partial fulfillment of the requirements \\ for the degree of \\ Doctor of Philosophy \\ in \\ Exercise Physiology \\ John M. Hollander, Ph.D., Chair \\ Stephen E. Alway, Ph.D. \\ Robert W. Brock, Ph.D. \\ Jefferson C. Frisbee, Ph.D. \\ Stephen Leonard, Ph.D. \\ Division of Exercise Physiology \\ Morgantown, West Virginia \\ 2010
}

Keywords: Diabetes, Mitochondria, Oxidative stress, Antioxidants 


\title{
ABSTRACT
}

\section{Mitochondrial Dysfunction in the Diabetic Heart: Cardioprotection from a novel mitochondrially-targeted therapeutic}

\author{
Erinne R. Dabkowski
}

\begin{abstract}
Cardiovascular complications leading to heart failure are the leading cause of death amongst both type 1 and type 2 diabetic patients. Increased reactive oxygen species (ROS) generation due to hyperglycemia and an enhanced fatty acid environment has been suggested to lead to mitochondrial dysfunction. Mitochondria are particularly susceptible to oxidative damage because the inner mitochondrial membrane (IMM) is the main site of ROS generation. Mitochondrial dysfunction is complicated by the fact that two spatially-distinct subpopulations of mitochondria reside in the heart. The subsarcolemmal mitochondria (SSM) are located at the cell periphery, while the interfibrillar mitochondria (IFM) situate along the myofibrils. It has been suggested that these two populations of mitochondria respond differently to various pathologies and physiological stimuli based upon their location in the cardiomyocyte. The goal of the following studies was to examine whether mitochondria located in different areas of the cell respond differently with type 1 and type 2 diabetes mellitus (DM). Type 1 DM was induced in 8 week old mice with a multiple low dose $(50 \mathrm{mg} / \mathrm{kg})$
\end{abstract} injection of Streptozotocin (STZ) administered for five days. Five weeks post hyperglycemia onset, hearts were excised and mitochondrial subpopulations were isolated. $\mathrm{Db} / \mathrm{db}$ (BKS.Cg- $m+/+\operatorname{Lepr}^{\mathrm{db}} / \mathrm{J}$ ) mice were utilized as a model of type $2 \mathrm{DM}$ and aged 18 weeks before hearts were extracted and mitochondrial subpopulations isolated. In the type 1 diabetic hearts, flow cytometry analysis of mitochondrial morphology indicated a decrease in size and internal complexity in the IFM with no changes in the SSM. On the other hand, in the type 2 diabetic hearts, the SSM size and complexity were decreased. Further, mitochondrial electron transport chain (ETC) activities and respiration were significantly decreased in the type 1 diabetic IFM while significantly decreased in SSM from type 2 diabetic hearts. Enhanced oxidative damage, assessed through lipid peroxidation by-products and nitrotyrosine formation, was specific to the IFM in the type 1 diabetic heart but enhanced in the SSM of the type 2 diabetic heart. Proteomic assessment revealed altered proteomic profiles in the type 1 diabetic IFM; however, changes were greater in the SSM of the type 2 diabetic heart. These data suggest for the first time, that depending on their location within the cell, mitochondria respond differently with type 1 and type 2 diabetic insults. The mitochondria located between the myofibrils are more dysfunctional in the type 1 diabetic heart; however, the mitochondria located beneath the sarcolemma are more dysfunctional in the type 2 diabetic heart. This emphasizes the importance of incorporating spatial location when examining mitochondrial dysfunction in the diabetic heart. Further, because the IMM is particularly susceptible to oxidative damage, we examined whether overexpression of a mitochondrially-targeted antioxidant enzyme, mitochondrial phospholipid hydroperoxide glutathione peroxidase (mPHGPx), provides 
protection in the diabetic heart. MPHGPx transgenic mice and controls were made diabetic through multiple low dose injections of STZ. Five weeks following hyperglycemia onset, in vivo analysis of cardiac contractile function revealed significantly decreased ejection fraction and fractional shortening in the diabetic heart, which was reversed with mPHGPx overexpression. MPHGPx overexpression in the diabetic heart increased mitochondrial ETC complex I, III, and IV activities in the diabetic IFM, with no differences on the SSM. Enhanced hydrogen peroxide production and lipid peroxidation were significantly attenuated in the diabetic IFM with overexpression of mPHGPX, with no changes in the SSM. MPHGPX is a unique antioxidant in that it has the ability to insert into the IMM and directly scavenge lipid hydroperoxides. Therefore, we assessed a phospholipid within the IMM essential for proper mitochondrial function, cardiolipin, and found preservation of cardiolipin in the diabetic IFM with overexpression of mPHGPx. These results indicate that mPHGPx overexpression provides cardioprotective benefits to the diabetic heart. Further, cardioprotection is associated with enhanced IMM function and preservation of a key IMM constituent, cardiolipin. These findings provide further rationale for the use of mPHGPx as a potential mitochondrially-targeted therapeutic that is capable of providing protection in the diabetic heart 


\section{LIST OF ABBREVIATIONS}

ADP

AGE

AMDCC

ANT

APOE

ATP

CAD

CL

cOX

DD

DIGE

DM

DNP

EDP

EDV

EM

EPR

ETC

FA

FAO

FFA

FSC

GLUT2

GPx

Grp75
Adenosine Diphosphate

Advanced Glycation End-products

Animal Models of Diabetic Complications Consortium

Adenine Nucleotide Transporter

Apolipoprotein $\mathrm{E}$

Adenosine Triphosphate

Coronary Artery Disease

Cardiolipin

Cytochrome C Oxidase

Diastolic dysfunction

Differential In Gel Electrophoresis

Diabetes Mellitus

Dinitrophenol

End Diastolic Pressure

End Diastolic Volume

Electron Microscopy

Electron Paramagnetic Resonance

Electron Transport Chain

Fatty Acid

Fatty Acid Oxidation

Free Fatty Acids

Forward Scatter

Glucose Transporter 2

Glutathione Peroxidase

Glucose Regulated Protein 75 


$\begin{array}{ll}\text { GSH } & \text { Glutathione reduced } \\ \text { GSSG } & \text { Glutathione oxidized } \\ \text { HAE } & \text { Hydroxyalkenals } \\ \text { HDL } & \text { High Density Lipoprotein } \\ \text { Hsp60 } & \text { Heat Shock Protein 60 } \\ \text { IDDM } & \text { Insulin Dependent Diabetes Mellitus } \\ \text { IFM } & \text { Interfibrillar Mitochondria } \\ \text { IMM } & \text { Inner Mitochondrial Membrane } \\ \text { I/R } & \text { Ischemia/Reperfusion } \\ \text { LC } & \text { Liquid Chromatography } \\ \text { LDL } & \text { Low Density Lipoproteins } \\ \text { LPO } & \text { Lipid Peroxidation } \\ \text { LV } & \text { Left Ventricle } \\ \text { MALDi } & \text { Matrix-Assisted Laser Desorption/ionization } \\ \text { MDA } & \text { Malondialdehyde } \\ \text { MnSOD } & \text { Manganese Superoxide Dismutase } \\ \text { MPHGPx } & \text { Mitochondrial Phospholipid Hydroperoxide Glutathione Peroxidase } \\ \text { MPTP } & \text { Mitochondrial Permeability Transition Pore } \\ \text { MUDPIT } & \text { Multi-Dimensional Protein Identification Technology } \\ \text { NIDDM } & \text { Insulin Independent Diabetes Mellitus } \\ \text { NO } & \text { Nitric Oxide } \\ \text { OM } & \text { Outer Membrane } \\ \text { OXPHOS } & \text { Oxidative Phosphorylation } \\ \text { PBS } & \text { Phosphate Buffered Saline } \\ \text { PHGPx } & \text { Phospholipid Hydroperoxide Glutathione Peroxidase } \\ \text { PSP } & \text { Peroxirexin } \\ \text { MPtolic Pressure }\end{array}$




\begin{tabular}{ll} 
PTM & Post Translational Modification \\
PUFA & Polyunsaturated Fatty Acids \\
ROS & Reactive Oxygen Species \\
SCX & Strong Cation Exchange \\
SD & Systolic Dysfunction \\
SeOH & Selenium Hydroxide \\
SOD & Superoxide Dismutase \\
SSC & Side Scatter \\
SSM & Subsarcolemmal Mitochondria \\
STZ & Streptozotocin \\
TCA & The Citric Acid cycle \\
Tg & Transgenic \\
TG & Triglycerides \\
TOF & Time Of Flight \\
UCP3 & Uncoupling Protein 3 \\
UQCRC2 & Ubiquinol Cytochrome C Reductase Core Protein 2 \\
VDAC & Voltage Dependent Anion Channel \\
WT & Wild Type \\
ZDF & Zucker Diabetic Fatty Rat \\
& \\
\hline
\end{tabular}




\section{TABLE OF CONTENTS}

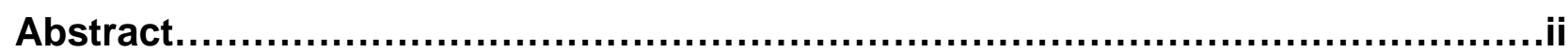

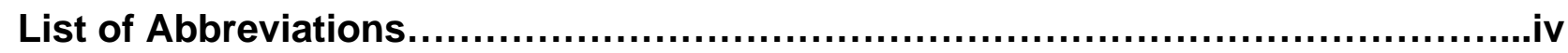

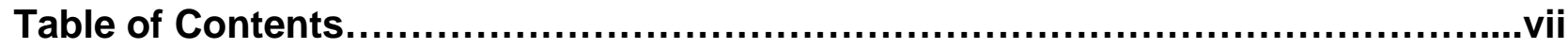

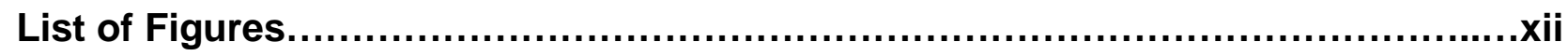

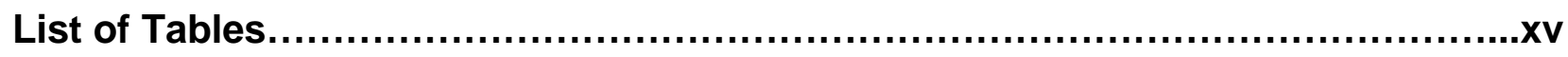

Specific Aims....................................................................................

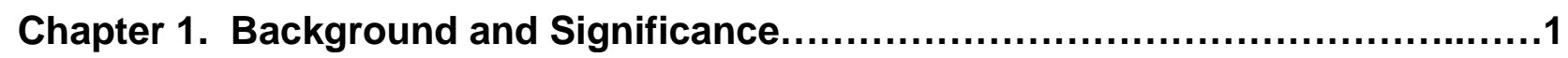

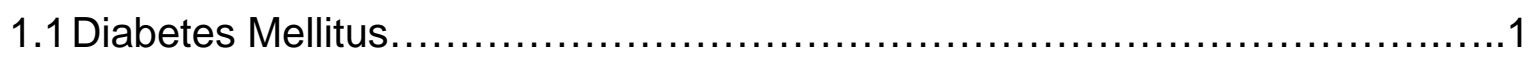

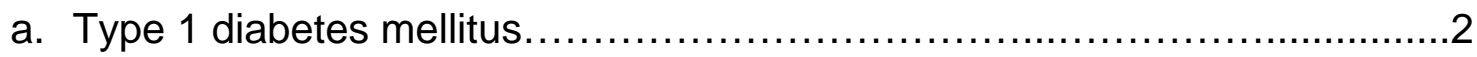

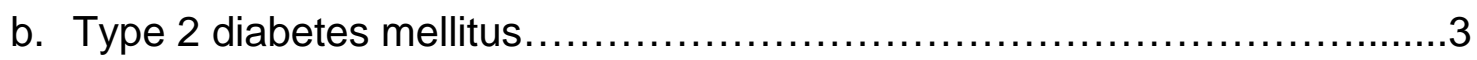

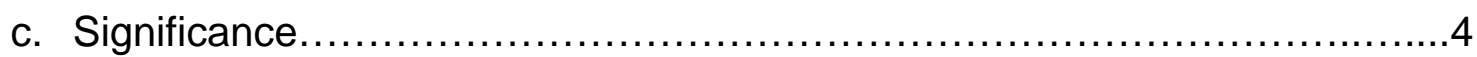

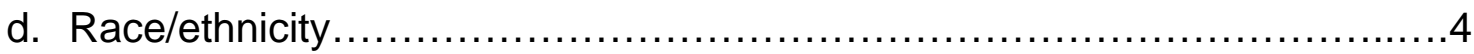

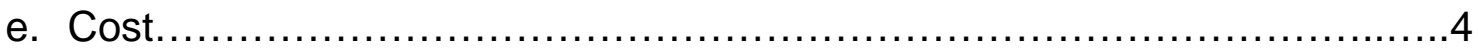

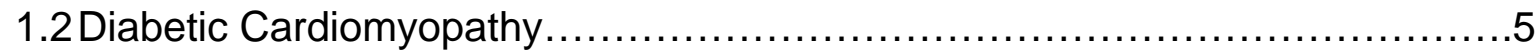

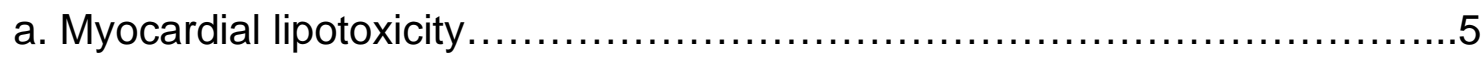

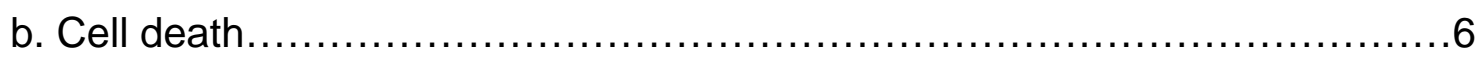

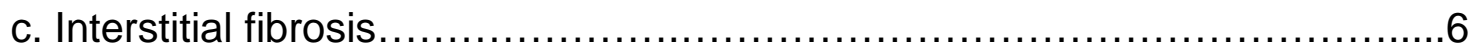

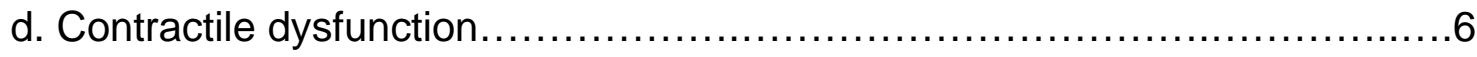

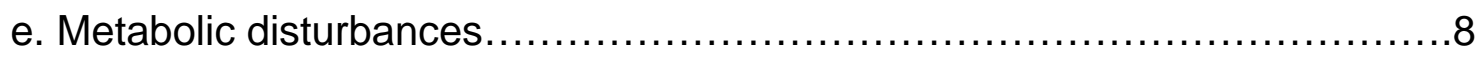

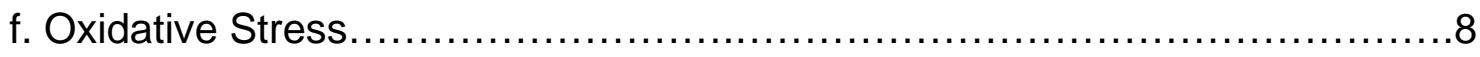

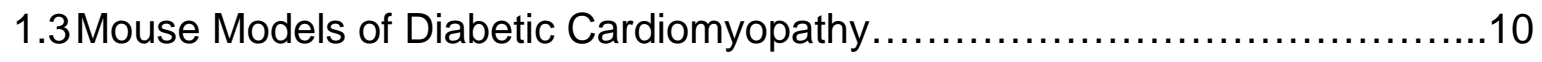

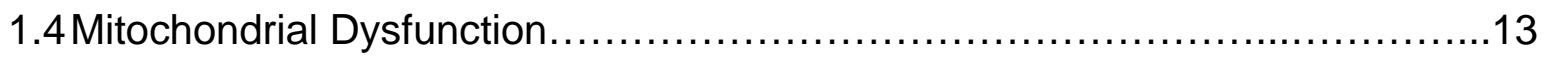


a. Mitochondrial number and morphology ...............................13

b. Mitochondrial energetics type 2 diabetes mellitus .........................14

c. Mitochondrial energetics type 1 diabetes mellitus.........................15

d. Calcium Regulation.........................................................

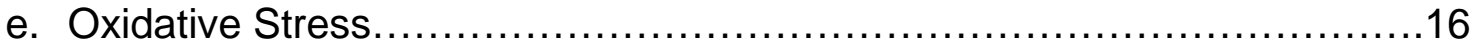

1.5 Mitochondrial Subpopulations..............................................

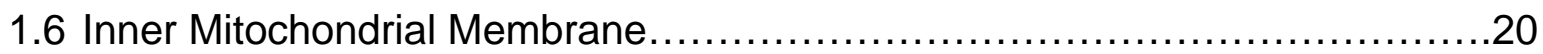

a. Mitochondrial ROS Production ........................................20

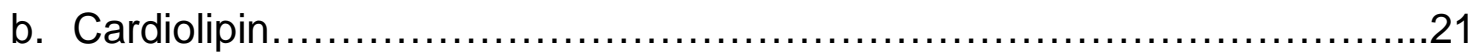

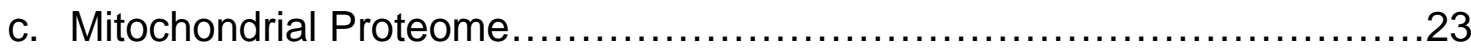

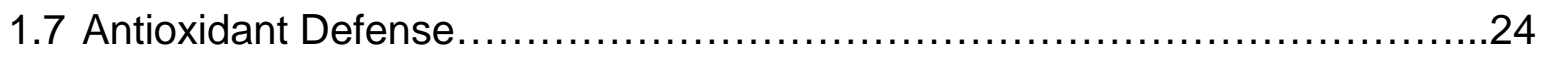

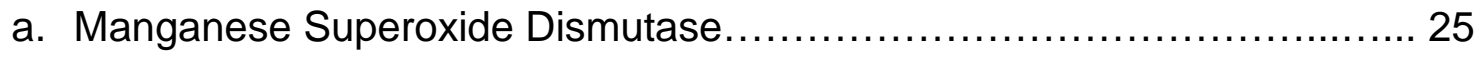

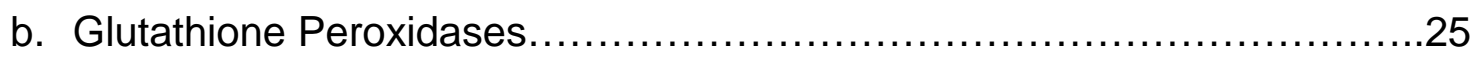

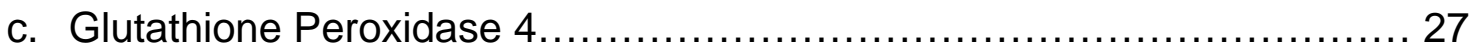

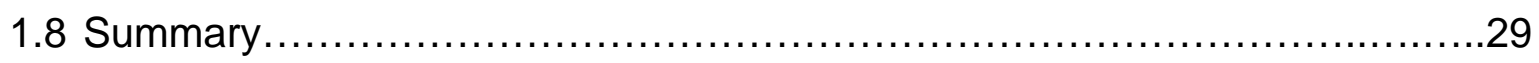

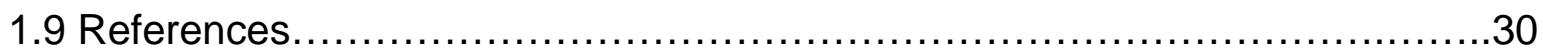

\section{Chapter 2. Diabetic cardiomyopathy-associated dysfunction in spatially distinct} mitochondrial subpopulations...................................................................

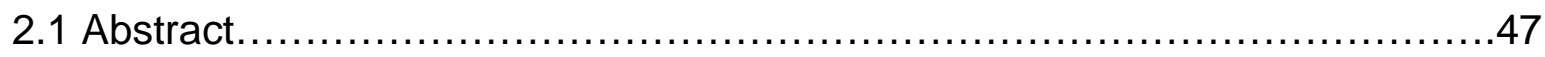

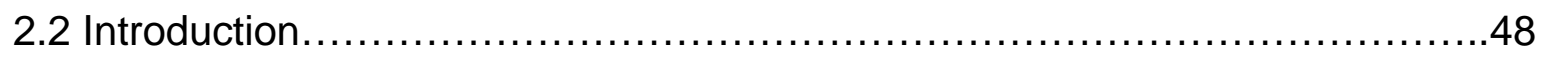

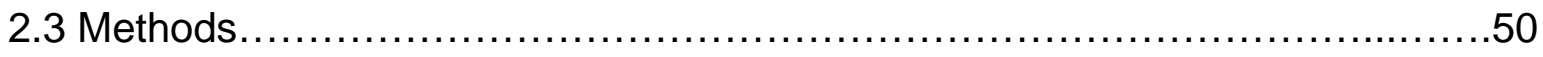

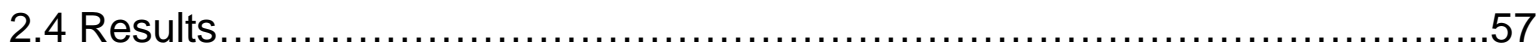

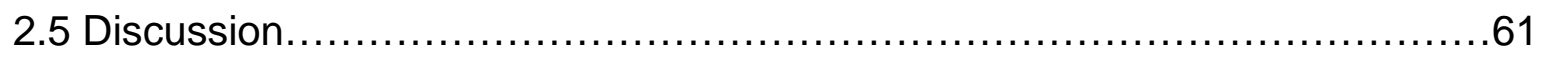

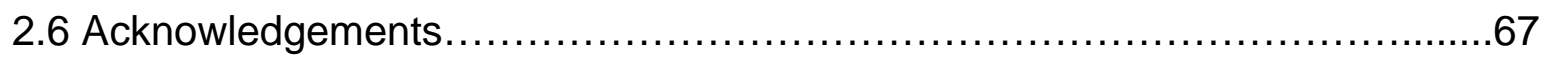




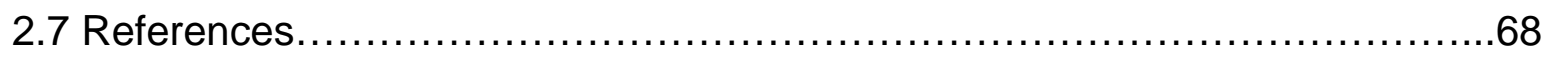

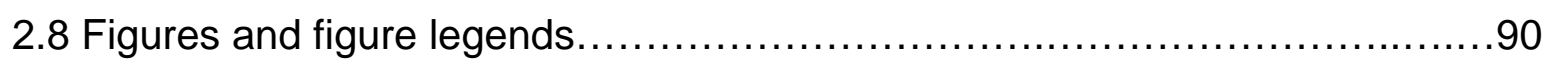

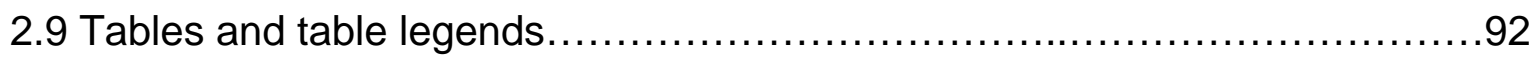

Chapter 3. Proteomic Alterations of Distinct Mitochondrial Subpopulations in the Type 1 Diabetic Heart: Contribution of Protein Import Dysfunction.

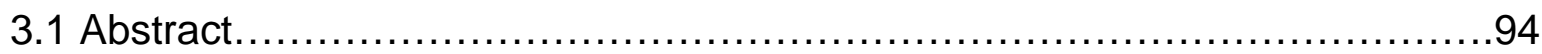

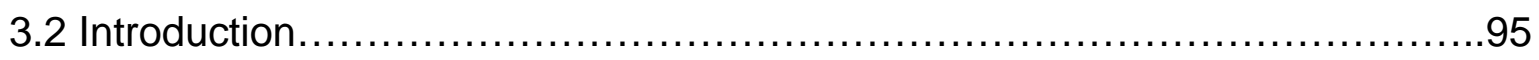

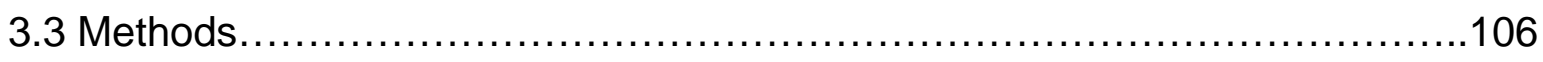

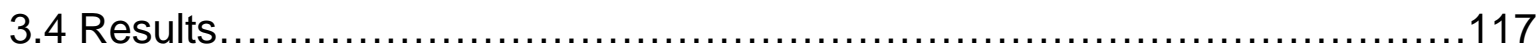

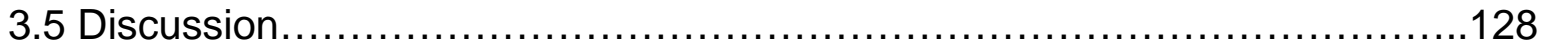

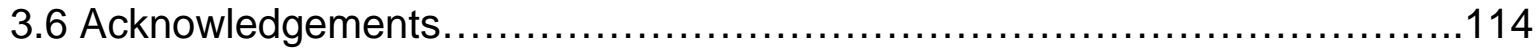

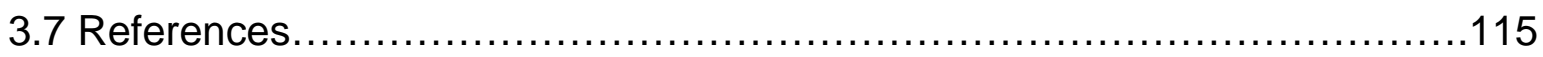

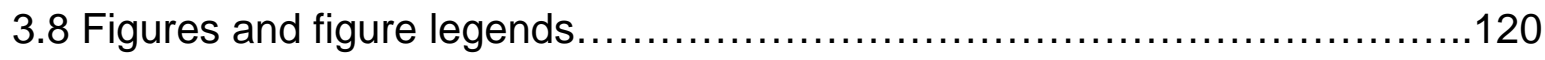

3.9 Tables and table legends...............................................

Chapter 4. Mitochondrial Dysfunction in the Type 2 Diabetic Heart is Associated with Alterations in Spatially-Distinct Mitochondrial Proteomes.........................148

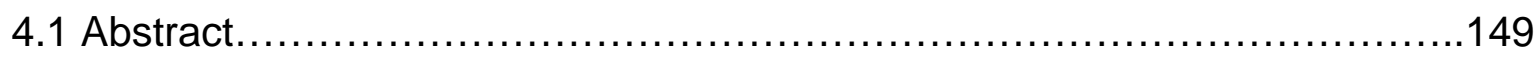

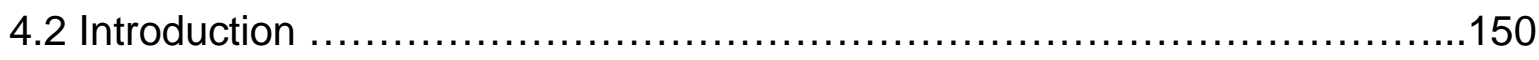

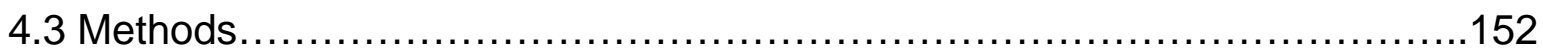

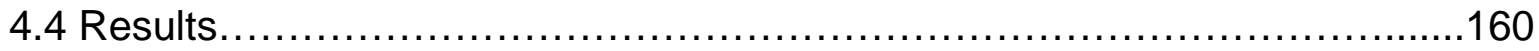

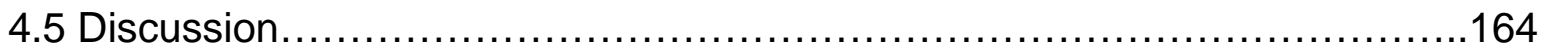

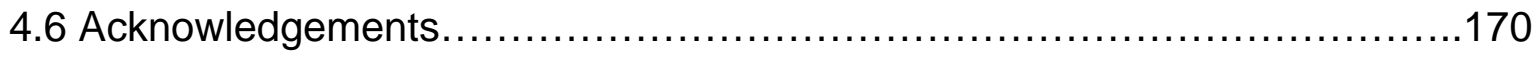

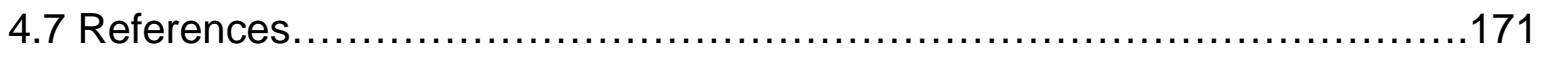

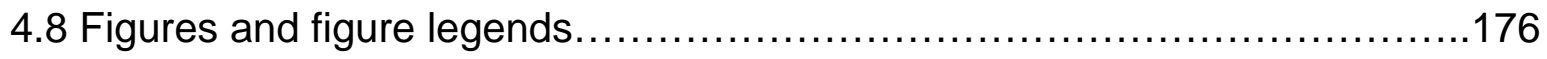

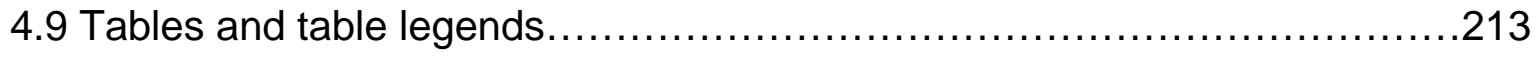


Chapter 5. Mitochondria-specific transgenic overexpression of phospholipid hydroperoxide glutathione peroxidase (GPX4) attenuates ischemia/reperfusion-

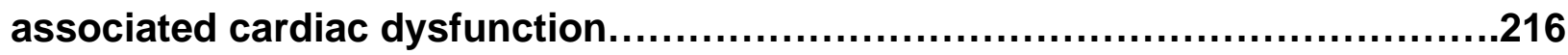

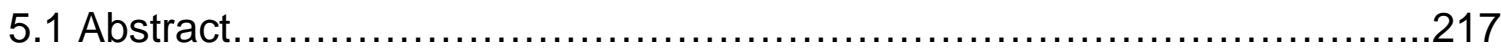

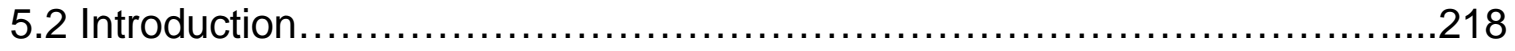

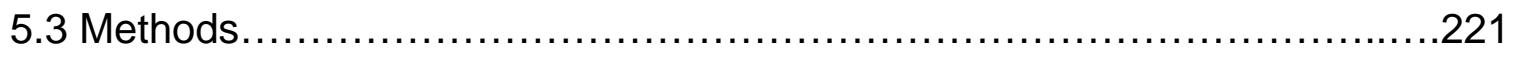

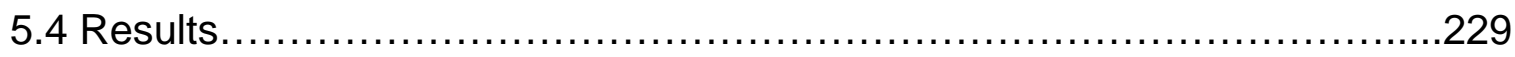

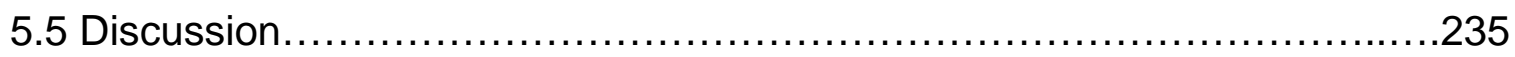

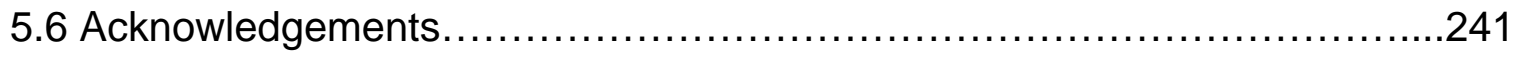

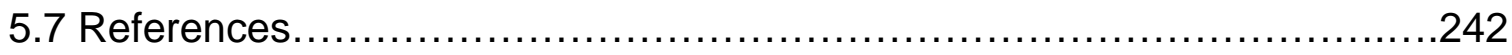

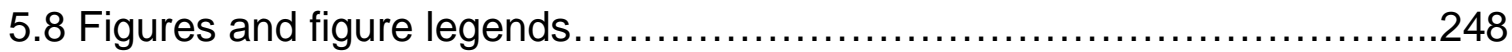

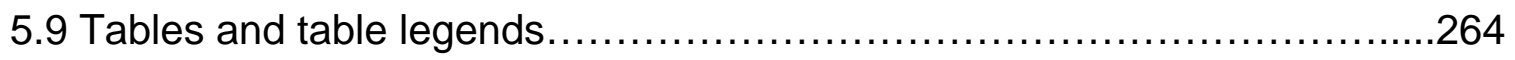

Chapter 6. Overexpression of Mitochondrial Phospholipid Hydroperoxide Glutathione Peroxidase (mPHGPx) Provides Protection in the Diabetic Heart....266

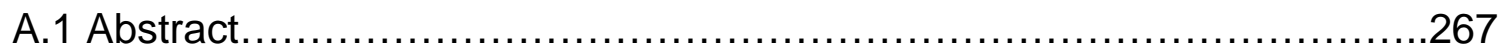

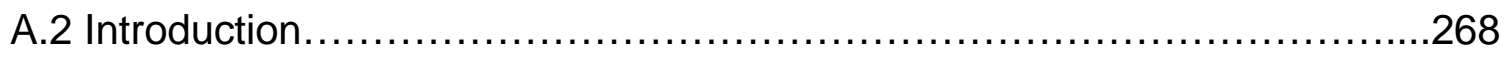

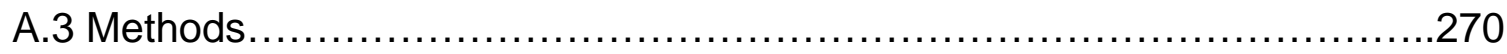

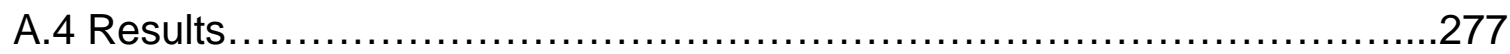

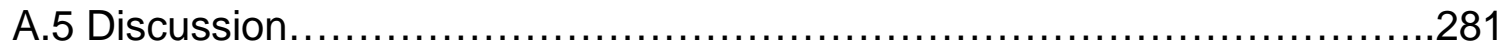

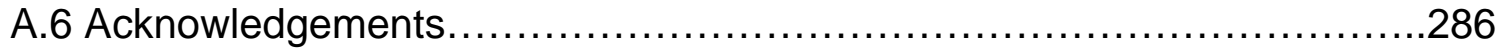

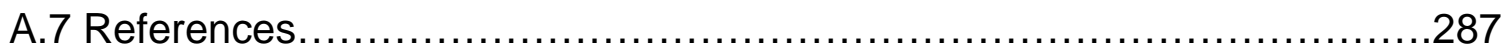

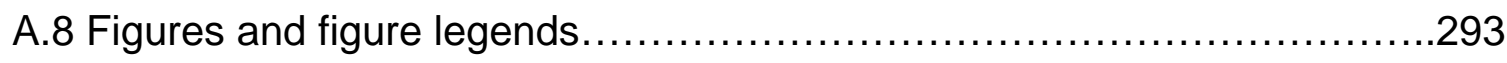

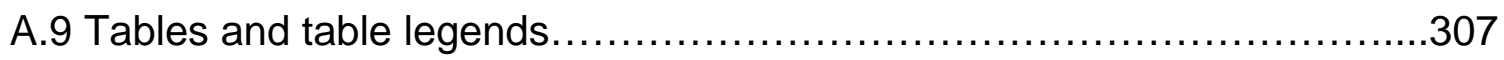


Chapter 7. General Discussion.

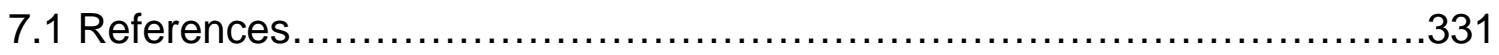

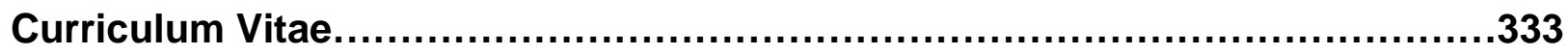




\section{LIST OF FIGURES}

\section{Chapter 1}

1.1 Mechanisms that contribute to cardiac dysfunction in the diabetic heart...........7

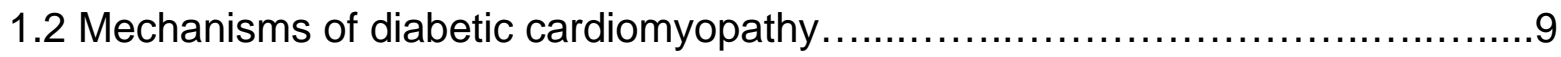

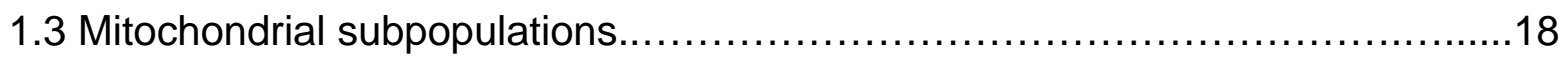

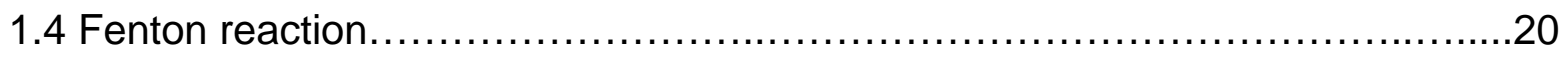

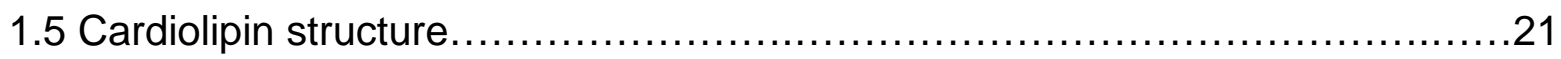

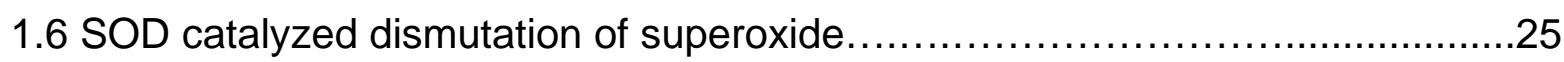

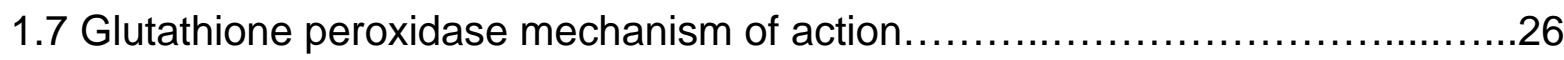

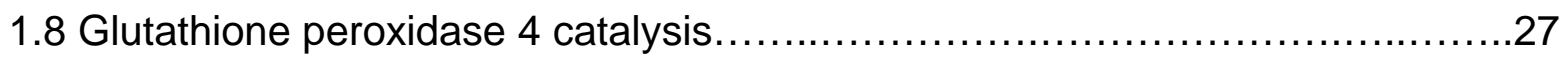

\section{Chapter 2}

2.1 Mitochondrial subpopulation morphological assessment...................76-78

2.2 Mitochondrial subpopulation respiration................................

2.3 Mitochondrial subpopulations ROS generation .............................81-83

2.4 Mitochondrial subpopulation protein nitrotyrosine content...................84-85

2.5 Mitochondrial subpopulation lipid peroxidation..............................86-87

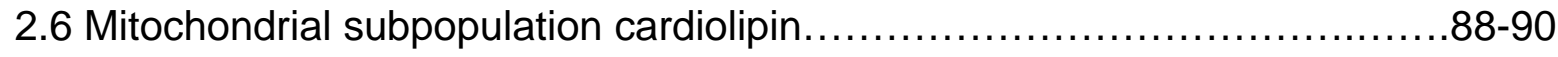

\section{Chapter 3}

3.1 Representative iTRAQ spectra ......................................120-121

3.2 Representative 2D-DIGE Gels .......................................122-124

3.3 pMITOGFP construct.................................................... 
3.4 Mitochondrial protein import.

3.5 Western blot analysis of protein import constituents

$129-130$

\section{Chapter 4}

4.1 Mitochondrial subpopulations size and internal complexity in WT and $\mathrm{db} / \mathrm{db}$.......

$176-178$

4.2 Respiratory capacity of mitochondrial subpopulations

in WT and $\mathrm{db} / \mathrm{db}$ hearts

$.179-183$

4.3 Flow cytometric analysis of mitochondrial subpopulation membrane potential

in WT and $\mathrm{db} / \mathrm{db}$ hearts.

4.4 ANT protein expression in mitochondrial subpopulations

from WT and db/db hearts.

$187-188$

4.5 Oxidative damage in mitochondrial subpopulations from

WT and db/db hearts

4.6 Representative iTRAQ spectra and Western

blot analysis

4.7 UCP3 protein expression in mitochondrial subpopulations

From WT and $\mathrm{db} / \mathrm{db}$ hearts $.201-202$

4.8 Representative iTRAQ spectra and Western

blot analysis 203-205

\section{Chapter 5}

5.1 MPHGPx transgenic mouse construction. $248-249$

5.2 MPHGPx protein expression $.250-251$ 
5.3 Antioxidant protein analysis. $252-253$

5.4 Cardiac contractile function following I/R ...............................254-255

5.5 Creatine kinase release following I/R ................................256-257

5.6 Total mitochondrial lipid peroxidation following I/R ......................258-259

5.7 Electron transport chain function following I/R .........................260-261

5.8 Mitochondrial subpopulations electron transport chain Function

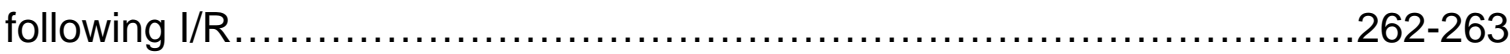

\section{Chapter 6}

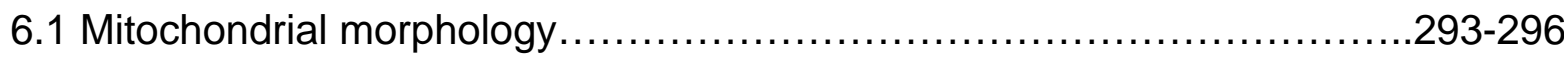

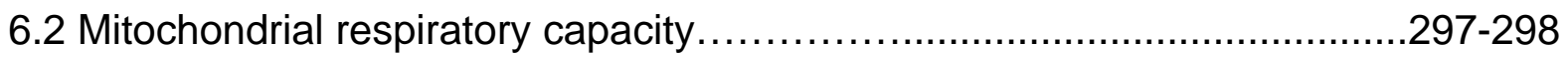

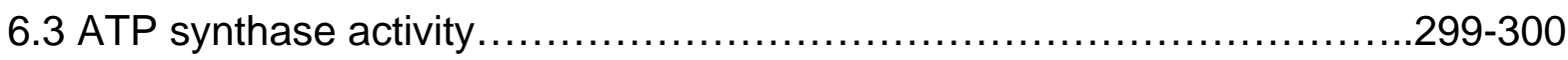

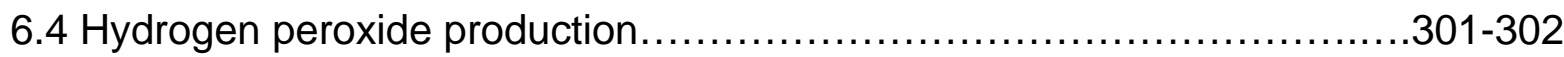

6.5 Lipid peroxidation by-products .......................................303-304

6.6 Mitochondrial membrane potential ....................................305-306

\section{Chapter 7}

7.1 Mitochondrial subcompartment protein abundance.............................325

7.2 Percentage of subcompartment proteomic changes...........................326

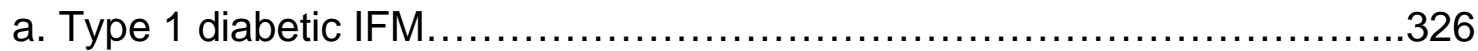

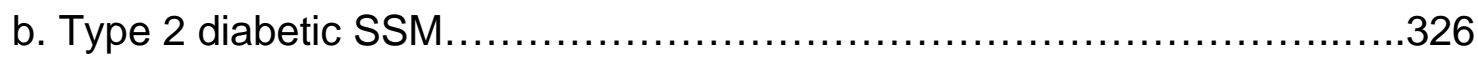

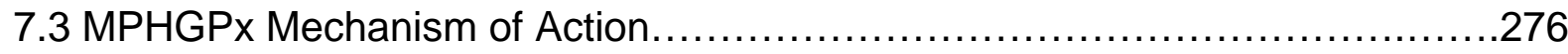




\section{LIST OF TABLES}

\section{Chapter 1}

1.1 Diabetes Mellitus characterization................................................

1.2 Cardiac phenotypes of rodent models of diabetic cardiomyopathy.

\section{Chapter 2}

2.1 Heart weight, body weight, contractile function and mitochondrial yields

\section{Chapter 3}

3.1 iTRAQ Proteomic analysis of mitochondrial subpopulations from Control and diabetic hearts.... $132-135$

3.2 2D-DIGE proteomic analysis of mitochondrial subpopulations from control and diabetic hearts

3.3 Post-translational modifications (PTM) in mitochondrial subpopulations from control and diabetic hearts

\section{Chapter 4}

4.1 Proteomic analysis of mitochondrial subpopulations from WT and $\mathrm{db} / \mathrm{db}$ hearts 195-197

4.2 Metabolic parameters 206-207

4.3 Cardiac contractile function from WT and $\mathrm{db} / \mathrm{db}$ hearts 208-209 4.4 Mitochondrial marker enzyme and protein yield 
4.5 Post-translational modifications in mitochondrial subpopulations from WT and db/db hearts

$.212-215$

\section{Chapter 5}

A.1 Mphgpx transgenic mouse screening ................................264-265

\section{Chapter 6}

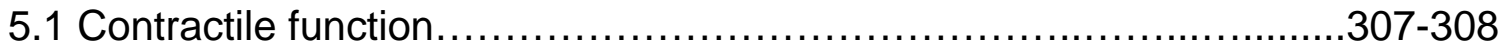

5.2 Electron transport chain activities ....................................309-310

5.3 Post-translational modifications........................................

5.4 Cardiolipin content...................................................... 


\section{SPECIFIC AIMS}

Heart failure is the leading cause of death among diabetic patients. Cardiovascular abnormalities, such as diabetic cardiomyopathy, contribute to the onset of heart failure with diabetes mellitus (DM) (1). Increased reactive oxygen species (ROS) and mitochondrial dysfunction have been suggested to contribute to the pathogenesis of DM $(1,12,14)$. Mitochondria are particularly susceptible to oxidative damage because the inner mitochondrial membrane (IMM) is the main site of ROS generation and is enriched with protein and lipid constituents that are easily oxidized (12). Mitochondrial dysfunction in the diabetic heart is complicated by the fact that there are two spatially distinct mitochondrial subpopulations interfibrillar (IFM) and subsarcolemmal (SSM). These two subpopulations are not only spatially distinct within the cell but have been suggested to respond differentially with various pathologies $(3,4$, $6-10,13)$. We hypothesize that one population may be more susceptible to diabetes-associated oxidative stress and ultimately contribute to overall mitochondrial and contractile dysfunction present with both type 1 and type 2 diabetes mellitus.

Overexpression of an antioxidant enzyme specific to the mitochondria may provide protection against increased ROS generation and subsequent disruption of the mitochondrial membrane. Mitochondrial phospholipid hydroperoxide glutathione peroxidase (mPHGPx) is an antioxidant enzyme specific to the IMM that is capable of scavenging peroxidized phospholipids such as cardiolipin hydroperoxide. MPHGPX reduces hydroperoxide groups on phospholipids, lipoproteins and cholesterol esters and may be beneficial in preserving mitochondrial membrane integrity and subsequent electron transport chain function $(\mathrm{ETC})(2,5,11)$.

Our long term goal is to mechanistically determine how mitochondrial dysfunction is associated with the pathogenesis of diabetes mellitus and to examine whether specifically targeted therapeutics are protective. The Objectives of this proposal are: (1) determine the effect of a type 1 diabetic insult on two distinct mitochondrial 
subpopulations; (2) examine whether mitochondrial subpopulations are differentially afflicted with type 2 diabetes mellitus; (3) to determine whether overexpression of a mitochondrial specific therapeutic is protective in mitochondrial subpopulations with type 1 DM. The Central Hypothesis of this proposal is that the IFM are more dysfunctional with type 1 and type 2 DM resulting from an enhanced oxidative environment and diminished membrane integrity. Further, we believe that overexpression of a mitochondrial specific antioxidant capable of preserving the IMM may serve as a potential therapeutic. Our central hypothesis is based on preliminary data in our laboratory suggesting that the IFM have diminished electron transport chain activities, enhanced ROS production, and increased oxidative damage resulting in disruption of the mitochondrial membrane with type $1 \mathrm{DM}$. Our preliminary data indicate that transgenic overexpression of mPHGPx will provide relief to the mitochondrial membrane by preserving constituents situated in the membrane such as the ETC and this will contribute to enhancing overall function with type $1 \mathrm{DM}$. Our rationale for the proposed research is to elucidate differential mechanisms and key components of mitochondrial dysfunction with DM that ultimately will aid in developing more spatially targeted therapeutics.

\section{Specific Aims:}

Aim 1: Determine the influence of a type 1 diabetic phenotype on specific mitochondrial subpopulations in the heart. To address Aim I, we will first isolate the subsarcolemmal (SSM) and interfibrillar (IFM) subpopulations in the diabetic heart to examine the effects of streptozotocin (STZ)-induced type 1 DM.

1.1 Overall subpopulation morphological and functional changes will be observed including size, complexity, lipid profile (cardiolipin) and total electron transport chain activities including ATP production. 
1.2 Oxidant production and subsequent oxidative damage will be assessed in the mitochondria by measuring ROS generation, lipid and protein oxidation.

1.3 Distinct proteomic profiles including specific proteomic modifications will be examined within the mitochondrial subpopulations with type $1 \mathrm{DM}$.

These findings will provide insight into the mechanism of mitochondrial dysfunction associated with diabetic cardiomyopathy and offer a spatially-specific therapeutic target within the mitochondrial subpopulations.

Working Hypothesis 1: We hypothesize that there will be an increase in oxidative stress and damage resulting in diminished mitochondrial membrane integrity accompanied by overall mitochondrial dysfunction in both diabetic mitochondrial subpopulations. More specifically, we believe that IFM will be more susceptible to damage with STZ-induced type 1 DM primarily due to their higher respiratory rates and subsequent oxidative environment. Due to the IFMs primary role in ATP production for contractile function, we believe the IFM will contribute to diabetic cardiomyopathyassociated dysfunction to a greater extent than the SSM.

\section{Aim 2: To determine the influence of a type 2 diabetic phenotype in two distinct} mitochondrial subpopulations. To address Aim 2, mitochondrial subpopulations, SSM and IFM, will be isolated from a db/db mouse hearts to examine whether they are differentially affected with a type 2 diabetic phenotype.

2.1Overall subpopulation morphological and functional changes will be observed including size, complexity, lipid profile (cardiolipin) and total electron transport chain activities including ATP production.

2.2Oxidant production and subsequent oxidative damage will be assessed in the mitochondria by measuring ROS generation, lipid and protein oxidation. 
2.3Distinct proteomic profiles including specific proteomic modifications will be examined within the mitochondrial subpopulations with a type 2 diabetic phenotype.

Working Hypothesis 2: We hypothesize that there will be an increase in oxidative stress and damage resulting in diminished mitochondrial membrane integrity accompanied by overall mitochondrial dysfunction in both type 2 diabetic mitochondrial subpopulations. More specifically, we believe that IFM population will be more susceptible with $\mathrm{a} \mathrm{db} / \mathrm{db}$ type 2 diabetic phenotype primarily due to their higher respiratory rates and subsequent oxidative environment. Due to the IFMs primary role in ATP production for contractile function, we believe IFM will contribute to diabetic cardiomyopathy-associated dysfunction to a greater extent than the SSM.

Aim3: To determine whether overexpression of a mitochondrial specific therapeutic is protective in mitochondrial subpopulations with a type 1 diabetic phenotype. To address Aim 3, a transgenic mouse line overexpressing mPHGPx will be employed in an effort to determine whether its presence can be protective with type 1 diabetes-induced mitochondrial and cardiac contractile dysfunction.

3.1Cardiac contractile function will be assessed in type 1 diabetic mPHGPX overexpressing mice.

3.2Overall subpopulation morphological and functional changes will be observed including size, complexity, phospholipid content (cardiolipin) and total electron transport chain activities including ATP production in type 1 diabetic mPHGPx overexpressing animals.

3.3Oxidant production and subsequent oxidative damage will be assessed through measuring ROS generation, and lipid and protein oxidation in mitochondrial subpopulations from type 1 diabetic mice with mPHGPx overexpression. 
3.4Distinct proteomic profiles including specific proteomic modifications will be examined within the mitochondrial subpopulations of type 1 diabetic mice with mPHGPx overexpression.

Working Hypothesis 3: We hypothesize that overexpression of mPHGPx will decrease oxidative stress and damage with type $1 \mathrm{DM}$. More specifically, overall mitochondrial structure and function will be protected through preservation of the IMM including cardiolipin, a phospholipid critical to membrane integrity and the stabilization of the ETC. Because our preliminary data has suggested that the IFM are at greatest risk for oxidative damage associated with DM, protection will be greatest in the IFM and will lead to a reduction of diabetic cardiomyopathy-associated contractile dysfunction. 


\section{References}

1. Flarsheim CE, Grupp IL, and Matlib MA. Mitochondrial dysfunction accompanies diastolic dysfunction in diabetic rat heart. The American journal of physiology 271 : H192-202, 1996.

2. Hollander JM, Lin KM, Scott BT, and Dillmann WH. Overexpression of PHGPX and HSP60/10 protects against ischemia/reoxygenation injury. Free radical biology \& medicine 35: 742-751, 2003.

3. Hoppel CL, Moghaddas S, and Lesnefsky EJ. Interfibrillar cardiac mitochondrial comples III defects in the aging rat heart. Biogerontology 3: 41-44, 2002.

4. Hoppel CL, Tandler B, Parland W, Turkaly JS, and Albers LD. Hamster cardiomyopathy. A defect in oxidative phosphorylation in the cardiac interfibrillar mitochondria. J Biol Chem 257: 1540-1548, 1982.

5. Imai $\mathrm{H}$ and Nakagawa $\mathrm{Y}$. Biological significance of phospholipid hydroperoxide glutathione peroxidase (PHGPx, GPx4) in mammalian cells. Free radical biology \& medicine 34: 145-169, 2003.

6. King KL, Young ME, Kerner J, Huang H, O'Shea KM, Alexson SE, Hoppel CL, and Stanley WC. Diabetes or peroxisome proliferator-activated receptor alpha agonist increases mitochondrial thioesterase I activity in heart. J Lipid Res 48: 1511-1517, 2007.

7. Leeuwenburgh C, Gurley CM, Strotman BA, and Dupont-Versteegden EE. Agerelated differences in apoptosis with disuse atrophy in soleus muscle. Am J Physiol Regul Integr Comp Physiol 288: R1288-1296, 2005.

8. Lesnefsky EJ, Moghaddas S, Tandler B, Kerner J, and Hoppel CL. Mitochondrial dysfunction in cardiac disease: ischemia--reperfusion, aging, and heart failure. $\mathrm{J} \mathrm{Mol}$ Cell Cardiol 33: 1065-1089, 2001.

9. Lesnefsky EJ, Tandler B, Ye J, Slabe TJ, Turkaly J, and Hoppel CL. Myocardial ischemia decreases oxidative phosphorylation through cytochrome oxidase in subsarcolemmal mitochondria. Am J Physiol 273: H1544-1554, 1997. 
10. Palmer JW, Tandler B, and Hoppel CL. Biochemical properties of subsarcolemmal and interfibrillar mitochondria isolated from rat cardiac muscle. The Journal of biological chemistry 252: 8731-8739, 1977.

11. Ran Q, Liang $\mathrm{H}$, Gu M, Qi W, Walter CA, Roberts LJ, 2nd, Herman B, Richardson $\mathrm{A}$, and Van Remmen $\mathrm{H}$. Transgenic mice overexpressing glutathione peroxidase 4 are protected against oxidative stress-induced apoptosis. The Journal of biological chemistry 279: 55137-55146, 2004.

12. Rolo $\mathrm{AP}$ and Palmeira $\mathrm{CM}$. Diabetes and mitochondrial function: role of hyperglycemia and oxidative stress. Toxicology and applied pharmacology 212: 167178, 2006.

13. Rosca MG, Vazquez EJ, Kerner J, Parland W, Chandler MP, Stanley W, Sabbah $\mathrm{HN}$, and Hoppel CL. Cardiac mitochondria in heart failure: decrease in respirasomes and oxidative phosphorylation. Cardiovasc Res 80: 30-39, 2008.

14. Shen X, Zheng S, Thongboonkerd V, Xu M, Pierce WM, Jr., Klein JB, and Epstein PN. Cardiac mitochondrial damage and biogenesis in a chronic model of type 1 diabetes. Am J Physiol Endocrinol Metab 287: E896-905, 2004. 


\section{Chapter 1:}

\section{Literature Review}




\subsection{Diabetes Mellitus}

Diabetes Mellitus (DM) is characterized by elevated levels of blood glucose resulting from defective insulin production, insulin action or a combination of both. DM can lead to a variety of complications and ultimately death if left untreated. There are several types of DM including type 1, type 2, gestational and DM resulting from genetic defects. Because DM is characterized by persistent hyperglycemia, the following criteria are used to diagnose DM (138).

\begin{tabular}{|c|c|c|}
\hline Condition & 2 hour glucose $(\mathrm{mg} / \mathrm{dl})$ & Fasting glucose $(\mathrm{mg} / \mathrm{dl})$ \\
\hline Normal & $<140$ & $<110$ \\
\hline $\begin{array}{c}\text { Impaired fasting glucose } \\
\text { Impaired glucose } \\
\text { tolerance }\end{array}$ & $<140$ & $\geq 110$ and $<126$ \\
\hline
\end{tabular}

Table 1.1 DM characterization (138)

The two most common types of DM are type $1(5-10 \%)$ and type 2 (90-95\%) DM.

\section{1a Type 1 DM}

Type 1 DM, also referred to as insulin-dependent DM (IDDM) or juvenile-onset DM, is characterized by loss of insulin-producing beta cells in the pancreas leading to diminished insulin production. Type $1 \mathrm{DM}$ is primarily considered to be immunemediated where beta cell loss is due to autoimmune attack (63). Type 1 DM is usually prevalent in children and young adults, although disease onset can occur at any age. In 
adults, type $1 \mathrm{DM}$ accounts for 5 to 10 percent of all diagnosed cases of DM. The main treatment for type $1 \mathrm{DM}$ is monitoring glucose levels and taking artificial insulin injections. Diabetic ketoacidosis is a complication that may occur predominantly with type $1 \mathrm{DM}$ due to the lack of insulin production and is characterized by high blood sugar, acidosis and high ketone body levels causing a low blood pH. Without treatment, diabetic ketoacidosis may be fatal (113). Risk factors for type 1 DM may be autoimmune, genetic, or environmental. No known way to prevent type 1 DM exists. Several clinical trials for the prevention of type $1 \mathrm{DM}$ are currently in progress or are being planned.

\section{1b Type 2 DM}

Type 2 DM, also referred to as insulin-independent DM (NIDDM), is by far majority of all diabetic cases (90-95\%). It is characterized by insulin resistance or reduced insulin sensitivity and sometimes diminished insulin secretion. The insulin resistance is believed to be due to a defect or lack of insulin receptors in cell membranes. In the early stages of type $2 \mathrm{DM}$, reduced insulin sensitivity causes an increase in insulin and glucose in the blood and this can be avoided through medications that increase insulin sensitivity or decrease glucose production (107). However, type 2 DM progresses, often insulin replacement is necessary due to lack of insulin production (26). Dyslipidemia is also characteristic with type $2 \mathrm{DM}$, though may be present with the type 1 population, and consists of elevation in triglyceride levels, low HDL cholesterol values, and small dense LDL particles. This lipoprotein pattern is associated with insulin resistance and is present even before the onset of DM. Small dense LDL particles are highly atherogenic because of their enhanced susceptibility to oxidative modification and increased uptake by the arterial wall. Type 2 DM is associated with older age, obesity, family history of DM, history of gestational DM, impaired glucose metabolism, inactivity and race/ethnicity (CDC, 2007). Type 2 DM can often be treatable with diet and exercise as there is a link between obesity, insulin resistance and developing type 2 DM (148). Severe long-term complications can result from untreated type $2 \mathrm{DM}$ and uncontrolled type $1 \mathrm{DM}$ including renal failure due to diabetic nephropathy, vascular disease (including coronary artery disease), vision 
damage due to diabetic retinopathy, loss of sensation or pain due to diabetic neuropathy, liver damage and heart failure from diabetic cardiomyopathy. These effects are ultimately linked to uncontrolled glucose levels, hyperglycemia, and altered substrate metabolism with both type 1 and 2 DM (13). Ultimately, heart failure and cardiovascular complications are the leading cause of death amongst diabetic patients (6).

\section{1c Significance.}

The increasing prevalence of DM is staggering. In 2007, it was stated that 23.6 million of the US population 20 years and older, approximately 7.8 percent of the population, have DM. Approximately 12 million of $11.2 \%$ of all men 20 and older have DM while 11.5 million of women have it. It is has been suggested that by the year 2030, incidence of DM is suspected to reach over 366 million (141). It is believed that these shocking statistics are mainly due to sedentary lifestyles and poor diets.

\section{1d Race/Ethnicity}

It is important to note that African Americans, Hispanic/Latino Americans, American Indians, and some Asian Americans and Native Pacific Islanders are at particularly high risk for type $2 \mathrm{DM}$ and its complications. Further, type $2 \mathrm{DM}$ amongst the listed Race/Ethnicities in children and adolescents is also being diagnosed more often (141).

\section{1e Cost}

In 2007, it was estimated that total costs (direct and indirect) from DM were 174 billion dollars. Direct medical costs were approximately 116 billion dollars, while indirect (disability, work loss and premature mortality) were 59 billion dollars. This emphasizes the impact DM has beyond physical well-being (141). 


\subsection{Diabetic Cardiomyopathy}

Coronary Artery disease (CAD) and hypertension play a major role in contributing to heart failure in type 2 diabetic patients. However, with both type 1 and type 2 diabetic patients there is a deterioration of heart function termed as diabetic cardiomyopathy which is independent of atherosclerosis. In a clinical setting, Rubler et al. (38) was first to acknowledge the existence of a diabetic cardiomyopathy in patients with congestive heart failure who had no evidence of coronary atherosclerosis, hypertension, valvular or congenital heart diseases. Regan et al (111) further provided evidence for diabetic cardiomyopathy in four adult diabetic patients without coronary artery disease. These patients were found to have increased left ventricle (LV) end diastolic pressure (EDP), normal LV end diastolic volume (EDV), decreased LV compliance, and decreased ejection fraction. Further epidemiologic studies, the Framingham study (111), also revealed an association of a specific cardiomyopathy with DM. They found that a 2.4fold greater incidence of heart failure in diabetic men and a 5.1-fold increase in women. These epidemiological studies indicated that diabetic patients can have a greater risk of developing heart failure independent of coronary artery disease, hypertension, serum cholesterol, and age suggesting that DM might be a cause of dilated cardiomypathy or heart failure.

\section{2a Myocardial Lipotoxicity}

It is well-established that type $2 \mathrm{DM}$ is associated with obesity which leads to myocardial liptoxicity. Lipotoxicity can further contribute to cell death and subsequent cardiac dysfunction. Enhanced myocardial tryglcyeride (TG) and cholesterol content have been shown in LV biopsies from human type 2 diabetic hearts. Further, Oil Red O staining of heart sections also revealed enhanced lipid deposition in type 2 diabetic hearts (10). An initial increase in myocardial fatty acid (FA) uptake and oxidation has been well documented in humans with both type 1 and type $2 \mathrm{DM}$, as well as in many different animal models (14). Enhanced FA can disrupt the integrity of the plasma and mitochondrial membranes by altering phospholipid content. This in turn can alter key signaling cascades as well as proper energy production from the mitochondria (10). 


\section{2b Cell death}

Myocardial biopsies of both type 1 and type 2 diabetic patients with heart failure independent of ischemia revealed enhanced apoptosis and necrosis within the heart (43). Increased cardiomyocyte apoptosis has also been described in STZ-induced rats (model of type $1 \mathrm{DM}$ ) (17) ob/ob and $\mathrm{db} / \mathrm{db}$ mice (model of type $2 \mathrm{DM}$ ) (5). The mechanism of enhanced cell death is not well understood, though it is has been suggested ROS-induced damage of mitochondria will induce cytochrome c release which inturn, activates the caspase 3 and the mitochondrial driven apoptotic pathway (17). Further, it has been suggested that hyperglycemia induces cell death through a Rac 1 mediated increase in NADPH and mitochondrial ROS in both the $\mathrm{db} / \mathrm{db}$ and STZ diabetic mouse hearts (129).

\section{2c Interstital Fibrosis}

Diabetic cardiomyopathy is also characterized by interstitial and perivascular fibrosis. Regan et al. (112) found enhanced collagen deposition around vessels and between myofibrils of diabetic patients. There was an increased accumulation of collagen type I and III fibers predominantly in the epicardial and perivascular regions, whereas type IV was found in the endocardial layer in human diabetic patients (3). Mechanisms of enhanced cardiac fibrosis in the diabetic heart are not entirely understood. One study suggested that TGFB1 receptor II, receptor for TGFB cytokine, was enhanced in the diabetic myocardium (4). Further, fibrosis has also been observed in STZ diabetic mice and was associated with enhanced PKCB1 (147). Interestingly, myocardial fibrosis was not found in $\mathrm{db} / \mathrm{db}$ mice (144). Ultimately, fibrosis in the heart can contributes to cardiac dysfunction the diabetic heart(10).

\section{2d Contractile Dysfunction}

Diastolic dysfunction (DD) is the most prominent defect with diabetic cardiomyopathy and it is characterized by a decrease in compliance and slower rates of relaxation $(128,156)$. Flow and tissue doppler analysis in type 1 and type 2 diabetic patients revealed diastolic dysfunction without coronary artery disease (12). Further DD 
was also found in animal models of type 2 and type $1 \mathrm{DM}$ including ob/ob, $\mathrm{db} / \mathrm{db}$ mice, ZDF rats and STZ mice $(12,100)$. Potential mechanisms contributing to DD include but are not limited to, lipid accumulation in the heart and altered calcium handling. Impaired calcium reuptake in ob/ob mice was associated with contractile dysfunction (62). Further, reduced contractility in isolated cardiomyocytes from $\mathrm{db} / \mathrm{db}$ mice were associated with enhanced diastolic sarcoplasmic reticulum SR-Ca2+ leak, reduced $\mathrm{Ca} 2+$ release, and lower peak systolic and diastolic calcium $(31,144)$. It has been suggested that DD precedes systolic dysfunction (SD). One study using PV loops to asses contractile function in STZ mice and Zucker diabetic fatty rats revealed systolic dysfunction in both models. STZ mice showed delayed LV relaxation, while the ZDF exhibited enhanced LV stiffness. Previously, we and others have published showing decreased systolic and diastolic function in STZ-induced type 1 diabetic mice through reduced rate of contraction and relaxation, and diminished contractility in the heart and in isolated cardiomyocytes $(28,128,131)$. Mechanisms of hyperglycemia induced cardiac dysfunction include but are not limited to: increased polyol pathway, increased advanced glycation end product (AGE) formation, increased protein kinase $c$ isoform expression, increased hexosamine pathway flux, altered cardiac metabolism, and lipotoxicity which leads to increases in oxidative stress $(40,69,107,117)$. Below is a suggested mechanisms of cardiac dysfunction in the diabetic heart (10).

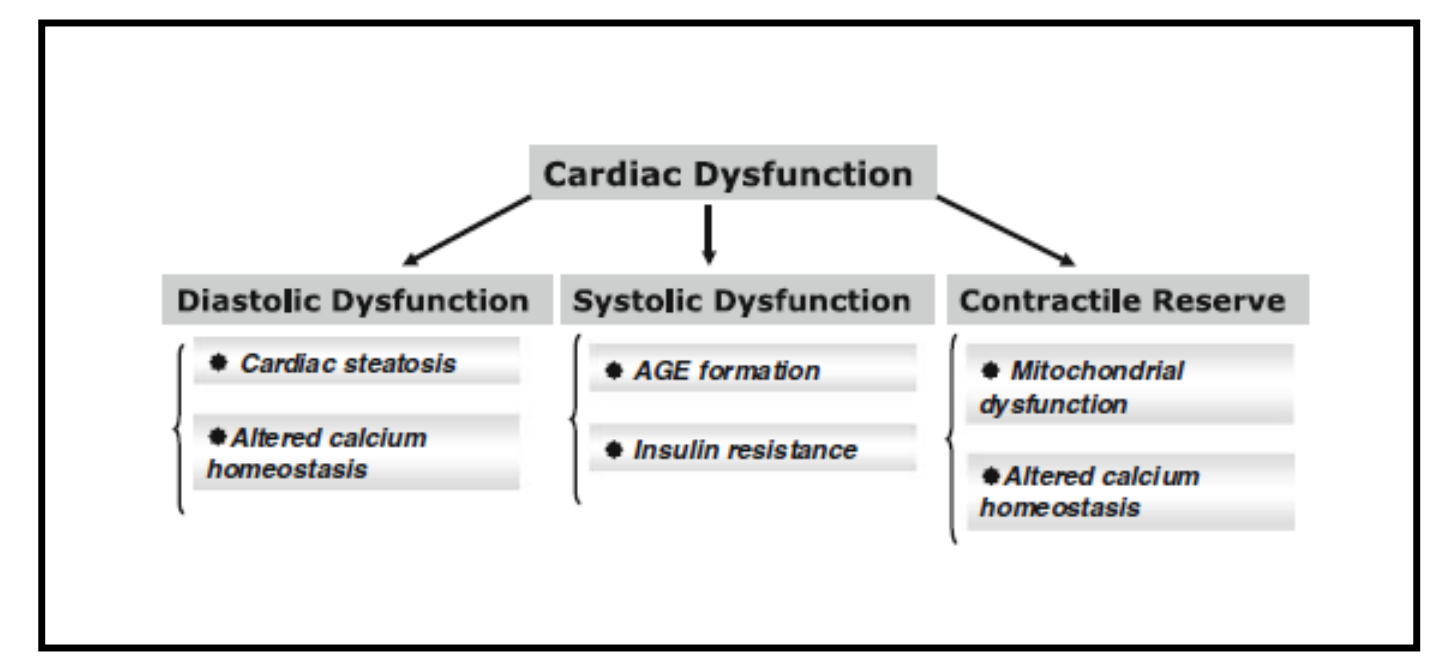

Figure 1.1 Mechanisms that contribute to cardiac dysfunction in the diabetic heart(10). 


\section{2e Metabolic Disturbances}

In both the type 1 and type 2 diabetic hearts there is altered substrate utilization (2). A decrease of glucose utilization in the heart leads to an initial enhanced FA utilization (149). Because FA are considered inefficient as substrates, increased FA oxidation in diabetic hearts is in combination with an increase in myocardial oxygen consumption which leads to reduced cardiac efficiency in rodent models (61) and in type 1 and type 2 diabetic patients. The enhanced FA utilization leads to a decrease in ATP which may play a larger role with type 2 DM due to higher overall free fatty acid (FFA) levels than in type 1 DM (36). It has been shown that inhibition of hypertriglyceridema in diabetic animals inhibited the development of diabetic cardiomyopathy (36). Elevated FA levels are believed to be one of the major contributing factors to the pathogenesis of DM. Enhanced FFA trigger cell death, diminish glucose oxidation, and lead to high oxygen requirements for FFA metabolism. This inturn leads to accumulation of toxic FFA intermediates. These toxic intermediates can pool within the mitochondria causing mitochondrial dysfunction and damage (149). Oxidative stress caused by these toxic intermediates has been suggested to disrupt calcium homeostasis. Diminished calcium sensitivity and decreased contractile proteins may further contribute to LV dysfunction (36).

\section{2f Oxidative Stress}

Oxidative stress, the imbalance between ROS formation and antioxidant defense, has been suggested to lead to altered myocyte morphology, function and protein content contributing to diabetic cardiomyopathy. Both human and animal studies have suggested that increased oxidative stress is associated with an accumulation of lipids and FA in the diabetic heart. Below is a suggested mechanism of FA-induced cardiac dysfunction through multiple facets including enhanced oxidative stress (Figure 1.2) (14). 


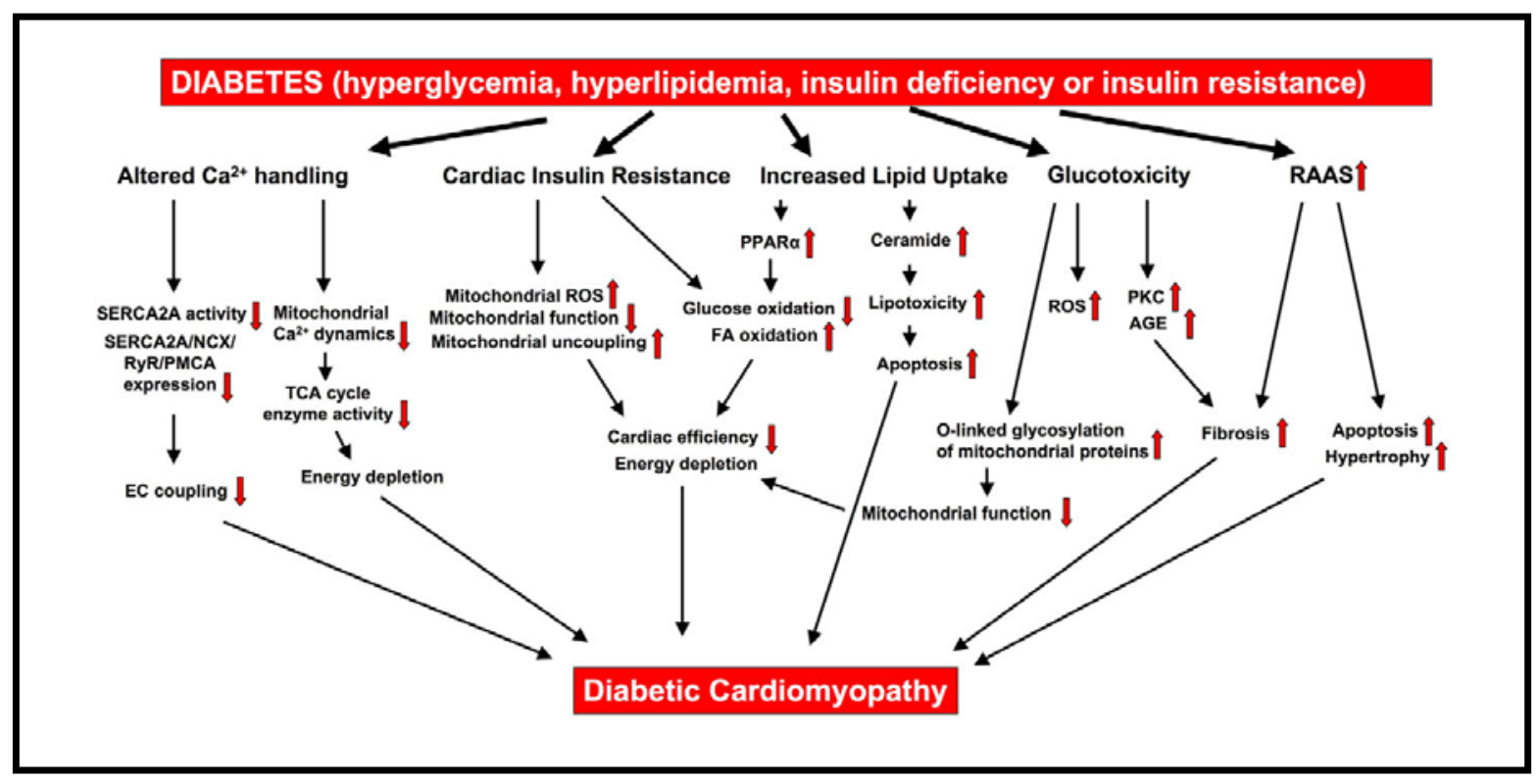

Figure 1.2 Mechanisms of Diabetic Cardiomyopathy (14).

Boudina et al. found that hydrogen peroxide production and subsequent oxidative damage to lipids was increased in the $\mathrm{db} / \mathrm{db}$ model of type $2 \mathrm{DM}$ (11). Similarly, our lab has suggested enhanced superoxide production in STZ-induced type 1 DM (28). These data suggest that in both the type 1 and type 2 diabetic hearts, there is evidence for an enhanced oxidative environment within the mitochondria which can disrupt proper oxidative phosphorlyation and adequate energy production. Further, though majority of ROS are generated from the mitochondria, other mechanism of ROS generation with DM exist such as NADPH oxidase (81). ROS can interact with nitric oxide (NO) and lipids to form nitrotyrosine and lipid hydroperoxides which are detrimental for cells and mitochondria (43). Further, it has also been suggested that hyperglycemia can impair and decrease the amount of antioxidants within the diabetic heart making it vulnerable to ROS-induced damage. Epstein et al. has shown that overexpression of antioxidants such as metallothionein, MnSOD and catalase in the heart offered protection against the development of type 1 diabetic cardiomyopathy $(83,154)$. 


\subsection{Mouse Models of Diabetic Cardiomyopathy}

Research on rodent models of type 1 and type $2 \mathrm{DM}$ and the use of genetic engineering techniques in mice have advanced our understanding of the development and progression of human diabetic cardiomyopathy (14). Rodents model are beneficial in studying underlying mechanisms of diabetic cardiomyopathy because they are resistant to atherosclerosis (without genetic alterations) which allows examination of diabetic cardiomyopathy dysfunction in diabetic models without confounding effects of atherosclerosis. There are many different rodent models of type $1 \mathrm{DM}$ and type $2 \mathrm{DM}$ in both rats and mice. Below is a table (Table 1.2) of multiple rodent models of type 1 and type $2 \mathrm{DM}$ and their phenotypes (14).

\begin{tabular}{|l|l|l|l|l|l|l|}
\hline & STZ & OVE26 & \multicolumn{1}{|c|}{ Akita } & ob/ob & db/db & ZDF \\
\hline Cardiac size & $=$ & & $\downarrow /=$ & $\uparrow$ & $\uparrow$ & $\uparrow$ \\
\hline Cardiac function & $\downarrow$ & $\downarrow$ & $\downarrow$ & $\uparrow / \downarrow$ & $\downarrow$ & $\downarrow$ \\
\hline Cardiac efficiency & $\downarrow$ & & $=$ & $\downarrow$ & $\downarrow$ & $=$ \\
\hline Mitochondrial energetics & $\downarrow$ & $\downarrow$ & $\downarrow$ & $\downarrow$ & $\downarrow$ & \\
\hline Lipid storage & $\uparrow$ & & & $\uparrow$ & $\uparrow$ & $\uparrow$ \\
\hline Fatty acid oxidation & $\uparrow$ & & $\uparrow$ & $\uparrow$ & $\uparrow$ & $\uparrow$ \\
\hline Glucose oxidation & $\downarrow$ & & $\downarrow$ & $\downarrow$ & $\downarrow$ & $\downarrow$ \\
\hline Ca ${ }^{2+}$ handling & $\downarrow$ & $\downarrow$ & $\downarrow$ & $\downarrow$ & $\downarrow$ & $=$ \\
\hline Oxidative stress & $\uparrow$ & $\uparrow$ & & $\uparrow$ & $\uparrow$ & \\
\hline & STZ & OVE26 & Akita & ob/ob & db/db & ZDF \\
\hline
\end{tabular}

Table 1.2 Cardiac Phenotypes of Rodent Models of Diabetic Cardiomyopathy(14).

We utilize the STZ model (Type 1 DM) and $\mathrm{db} / \mathrm{db}$ model (Type $2 \mathrm{DM}$ ) for experimentation. STZ is a glucosamine-nitrosourea antibiotic that is similar to the structure of glucose and is taken up through GLUT2 glucose transporter in the beta cells (high GLUT2 receptors) and causes the akylation of DNA (122). DNA damage induces activation of poly ADP-ribosylation which leads to depletion of cellular NAD+ and ATP. Enhanced ATP dephosphorylation after STZ administration offers a substrate 
for xanthine oxidase resulting superoxide radical formation. STZ releases toxic amounts of NO that inhibits aconitase activity and contributes to DNA damage. Ultimately, beta cells undergo necrosis and insulin production is disrupted. We utilize the STZ protocol established by the Animal Models of Diabetic Complications Consortium (AMDCC) which recommends mulitiple low-dose $(50 \mathrm{mg} / \mathrm{kg}$ ) injection of STZ spread out over 5 consecutive days (www.amdcc.org). Rodents typically develop hyperglycemia 7-14 days post first injection of STZ and show increased serum FAs, triglycerides, cholesterol and glucose levels with very low levels of insulin (14). Many studies have reported diminished systolic and diastolic function which progresses with duration of STZ induced DM. Nielsen et al. reported decreased rates of circumferential shortening and fractional shortening with echocardiographic analyses (95). Further, reduced LV systolic pressure and decreased rates of contraction were also observed (68). DD has been further shown through enhanced LV diastolic pressure and abnormal mitral inflow and pulmonary venous flow (139). We have shown decreases in rate of contraction, relaxation and enhanced developed pressure at five weeks post hyperglycemia onset (28). Further, studies have also revealed enhanced oxidative stress through both the mitochondria and through NADPH oxidase, in the STZ mouse heart. STZ hearts have also shown changes to calcium handling including disruption of mitochondrial calcium energetics (14). Importantly, STZ-induced diabetic cardiomyopathy can be reversed with regular insulin injections emphasizing the lack of insulin and glucose utilization being the cause of cardiac abnormalities in these mice not the STZ drug itself (145). The most important advantages of the STZ model are that DM can be easily induced in mice and rats at multiple time points (different ages) and genetically altered mice can be easily examined with a diabetic phenotype, allowing researchers to study specific mechanisms of dysfunction underlying diabetic cardiomyopathy in hopes of identifying key targets. Other models of type $1 \mathrm{DM}$ that are commonly used are the Akita mouse and the OVE26 mouse. They differ from the STZ model in that they are genetically engineered mice that are diabetic from birth (TABLE 2). Ove26 mouse model was generated in 1989 by Epstein et al (35). Overexpression of the $\mathrm{Ca}+2$ binding protein calmodulin in beta cells led to insulin-deficient DM within the first week of life due to beta cell damage. Ove26 have increased serum TG levels, 
reduced insulin levels, and are able to live approximately 1 to 2 years. In the OVE26 mouse, contractility analysis of isolated cardiomyocytes revealed altered peak shortening, prolonged time to peak shortening, prolonged time re-lengthening, and reduced maximal velocities of shortening and re-lengthening (33). However, unlike the STZ model, no significant differences in contractile function were found in Langendorff perfusions of OVE26 diabetic hearts (83). Although the OVE26 mouse allows for examination of type $1 \mathrm{DM}$ independent of utilizing a potentially toxic drug (STZ), DM exists initially at a very early state in postnatal development which may alter cardiac development in a way that does not truly mimic type $1 \mathrm{DM}$. The Akita mouse model of type 1 DM was more recently discovered by Yoshiolka et al (155). This mouse develops DM due to a single base pair substitution in the INS2 gene resulting in improper folding of proinsulin which leads to protein aggregate-induced ER stress in the pancreas leading to B-cell destruction (155). Akita mice develop hyperglycemia at 5 to 6 weeks of age and have elevated serum fatty acids and triglycerides (15). In vivo analysis of contractile function through echocardiography did not reveal cardiac contractile abnormalities (85) in the Akita mouse, suggesting cardiac contractile dysfunction is not apparent in this model. However, FAO rates are enhanced while glucose oxidation rates are decreased (15). Further, mitochondrial dysfunction is present including alterations to FAO and OHPHOS proteins (16).

Several mouse models of type $2 \mathrm{DM}$ exist including ob/ob and $\mathrm{db} / \mathrm{db}$ mice. Ob/ob mice develop DM due to recessive mutations in the obesity gene (ob or Lep). In ob/ob mice, obesity and DM result from leptin deficiency and the inability to suppress appetite (42). By 4 weeks of age, ob/ob mice (C57BL/6) are moderately obese, hyperinsulinemic and altered glucose tolerance (13). By 15 weeks of age, ob/ob mice are obese and develop type $2 \mathrm{DM}$. Ob/ob mice further develop cardiac hypertrophy but have little impairment in systolic function. Further, in isolated working heart perfusions, contractile function is not impaired in the ob/ob mice (14). Therefore, in our lab we utilize $\mathrm{db} / \mathrm{db}$ mice because we are interested in cardiac abnormalities associated with type $2 \mathrm{DM}$. Db/db mutations are another model of obesity and type $2 \mathrm{DM}$ that develops because of lack of leptin action (24). Leptin action is impaired in $\mathrm{db} / \mathrm{db}$ mice due to 
dysfunctional leptin receptors. Insertion of a premature stop codon within the $\mathrm{db}$ transcript leads to the long form of the leptin receptor being replaced with the short-form isoform. The long form is responsible for leptin action in the hypothalamus to regulate appetite and body weight and lack of this form leads to obesity(24). Db/db mice develop severe type 2 DM by 8 weeks of age and have early hyperinsulinemia, enhanced fatty acids and triglycerides (13). Further, contractile abnormalities have been observed in $\mathrm{db} / \mathrm{db}$ (C57BL/Ks) mice. Db/db mice assessed with echocardiography develop cardiac hypertrophy, reduced fractional shortening and velocity of circumferential shortening (126). Further, cardiac output, LV-developed pressure and cardiac power are all reduced in isolated, working $\mathrm{db} / \mathrm{db}$ hearts (14). Isolated langendorff perfused $\mathrm{db} / \mathrm{db}$ hearts have similar contractile dysfunction as seen through decreased rates of contraction, peak systolic pressure, rate pressure product and developed pressure (8). Diastolic function is also impaired in $\mathrm{db} / \mathrm{db}$ mice as assessed through echocardiography or MRI analysis (136). Mitochondrial dysfunction has also been observed in $\mathrm{db} / \mathrm{db}$ mice along with enhanced mitochondrial superoxide formation and a subsequent increase in oxidative damage by-products (11).

\subsection{Mitochondrial Dysfunction and DM}

There is evidence that mitochondrial dysfunction is associated with type 1 and type 2 DM. Altered substrate utilization, oxidative stress, mitochondrial morphology, mitochondrial number and calcium energetics are all altered in the type 1 and 2 diabetic setting. Mitochondrial dysfunction encompasses a wealth of mitochondrial defects including but not limited to, alterations to mitochondrial bioenergetics, altered mitochondrial number and morphology, calcium dysregulation and enhanced oxidative stress.

\section{4a Mitochondrial number and morphology}

Utilizing Electron Microscopy (EM), biopsies of skeletal muscle from subjects with type $2 \mathrm{DM}$ and obesity revealed mitochondria of smaller size and a decrease in density of the mitochondria located beneath the sarcolemma $(71,114)$. Type $1 \mathrm{DM}$ is also 
associated with alterations in mitochondrial morphology. Skeletal muscle mitochondria of STZ rats showed a loss of cristae and an increase in lipid droplets surrounding the mitochondria (19). Alloxan induced diabetic rats revealed a decrease in mitochondrial number in liver and heart, with mitochondrial swelling and disrupted mitochondrial membranes and cristae (87). Further, Epstein et al found swollen mitochondria in the hearts from OVE26 mice (131). A recent study using EM to examine heart mitochondria of Akita mice, revealed reduced cristae density and larger mitochondria than the wildtype (WT) mice (16). On the other hand, the same group (34) revealed that mitochondrial number was increased and that mitochondria were smaller in cardiac muscle of $\mathrm{db} / \mathrm{db}$ mice compared with WT. Interestingly, we have shown decreases in size and internal complexity of those mitochondria located along the myofibrils in the STZ-induced type 1 diabetic heart. Taken together, morphologic alterations of the mitochondria seem to differ depending on duration and model of DM being examined. Variations between the studies are mostly due to differing methods used for assessment.

\section{4b Mitochondrial energetics with type 2 DM mellitus}

Studies of type 2 diabetic human subjects and rodents provide evidence for deficiencies in oxidative phosphorylation in mitochondria from skeletal muscle. Kelley et al. (71) studied mitochondria isolated from human skeletal muscle biopsy specimens obtained from type 2 diabetic, obese, and lean individuals. They found reduced NADH oxidoreductase and reduced citrate synthase activity in the mitochondria from the diabetic and obese patients as compared to lean subjects. Mitochondrial respiration has been also been assessed in human muscle in vivo by using ${ }^{13} \mathrm{C}$ nuclear magnetic resonance (NMR) to assess TCA flux rates along with ${ }^{31} \mathrm{P}$ NMR to assess phosphorylation of ADP. These studies showed that skeletal muscle oxidative phosphorylation was impaired in individuals with type $2 \mathrm{DM}$. The authors suggested that defects in oxidative phosphorylation were associated with increased intramyocellular lipid (110). Further, Nielsen et al. showed enhanced lipid deposition in the sarcolemmal region of the skeletal muscle from type 2 diabetic patients (94). Several groups have suggested decreased mRNA expression of several genes 
associated with oxidative phosphorylation such as genes regulated by PGC-1a (90). Examination of protein expression in skeletal muscle from type 2 diabetic patients revealed decreases in ATP synthase and creatine kinase $B$ which contribute to overall ATP synthesis (56). Mitochondrial dysfunction has also been observed in various rodent models of type 2 DM. Boudina et al reported decreased respiration with glutamate/malate and palmitoyl-carnitine as substrates in saponin-permeabilized heart muscle fibers isolated from $d b / d b$ mice compared with lean controls (11). These authors also reported decreases in expression of $F_{1} \alpha$-subunit of ATP synthase and an increase in fatty acid-induced proton leak. Utilizing a high-fat feeding model, Koves et al. (74) showed that high-fat feeding was associated with decreased TCA proteins in skeletal muscle and an inability of mitochondria to switch from fat substrate utilization to glucose utilization. This suggests that high-fat fed rodents have increased rates of betaoxidation but are not capable of dealing with enhanced fatty-acid substrates supply.

\section{4c Mitochondrial energetics in Type $1 \mathrm{DM}$}

Early studies with type 1 DM revealed decreased respiration with complex I and complex II substrates in liver and skeletal muscle mitochondria isolated from rodents made diabetic with STZ (48). Several studies in rodents suggested that respiration is impaired in mitochondria isolated from heart and brain mitochondria $(28,39,91)$. Recently Bugger et al. examined heart mitochondria of Akita mice and reported decreased ATP synthesis and decreased state 3 respiration with complex I substrates pyruvate and glutamate, but no differences with a fatty acid substrate, palmitoyl carnitine (15). Sevitz et al reported a decrease in state 3 respiration, ADP/O ratio and ATP synthesis rate in mitochondria from heart and gastrocnemius muscle from STZ diabetic rats (54). Interestingly, these findings were more pronounced in 8-week than in 2-week diabetic rats suggesting the importance of examining multiple time points to truly understand the mechanism of pathogenesis with type 1 DM. In a human study, analysis of skeletal muscle mitochondria that were subject to discontinuation of insulin treatment resulted in a decrease in ATP production (70). These studies suggest that respiration and subsequent ATP production in skeletal muscle and heart mitochondria are dysfunctional in the type 1 diabetic setting. 


\section{4d Calcium Regulation}

Under conditions of high matrix calcium concentrations and enhanced ROS, the permeability transition pore (PTP) opens disrupting the IMM function. This further triggers disruption of ionic homeostasis and uncoupling of oxidative phosphorlyation which results in cell death (119). Oliveira et al. observed reduced mitochondrial calcium uptake and enhanced mitochondrial PTP (mPTP) opening in heart mitochondria from STZ-treated rats (99). PTP opening due to calcium accumulation and enhanced ROS has been attributed to structural changes in the IMM lipids which inturn disrupts function and structure of the respiratory chain. Oliveria et al. further reported increased sensitivity to calcium-triggered reduction in membrane potential and prevention of this with cyclosporine A suggested that this was due to enhanced mPTP opening (99). Further, our lab has suggested that the interfibrillar mitochondria had decreased membrane potential, enhanced propensity for mPTP opening and enhanced cytochrome c release in 10 week old STZ-treated mice (150). Taken together, these studies suggest that DM-induced changes in calcium regulation and ROS may result in damage to the IMM and trigger apoptosis.

\section{4e Oxidative Stress}

Enhanced ROS production and oxidative damage have been suggested to play a critical role in the pathogenesis of type 1 and type 2 DM. Many studies have shown that elevated glucose and free fatty acids levels present in the diabetic state drive the formation of ROS $(32,96,152)$. This inturn, decreases mitochondrial function and contributes to many of the complications seen with DM. It is believed that enhanced substrate flux through the mitochondria enhances electron leak and subsequently ROS formation. Oxidative stress occurs when ROS are generated in excess through the reduction of oxygen and inadequate antioxidant defense. ROS include superoxide radicals, hydroxyl radicals, hydrogen peroxide, nitric oxide (NO), and peroxynitrite (151). ROS production is also increased with DM due to an increase in circulating FFA causing a lipotoxic environment within the mitochondria (149). For example, pooled neutral fatty acids within the mitochondria due to the inability to utilize efficiently are more 
susceptible to oxidative damage. Oxidative modification to these lipids can initiate greater oxidative damage to proteins and lipid constituents within the IMM contributing to mitochondrial dysfunction (149). Excess ROS production can lead to oxidation of lipids, DNA, and proteins. Levels of markers of lipid peroxides, such as 8-isoprostaglandin $\mathrm{F} 2 \alpha(55)$, conjugated dienes, and lipid hydroperoxides (88), are increased with type $1 \mathrm{DM}$ while antioxidant capacity is reduced (88). Because the IMM is the main site of ROS production, it is particularly susceptible to oxidative damage. An increase in superoxide production in the mitochondria has been suggested to directly inhibit ETC complex activities causing overall diminished mitochondrial respiration (131). Abel et al. suggested that mitochondrial uncoupling, due to enhanced ROS generation and lipid peroxidation by-products, contributed to enhanced oxygen consumption without an increased in ATP. Hence, reduced cardiac efficiency was seen in the $\mathrm{db} / \mathrm{db}$ and ob/ob hearts (11).

\subsection{Mitochondrial Subpopulations}

There are two spatially and biochemically distinct mitochondrial subpopulations located within the cardiomyocyte (101). These two subpopulations are termed subsarcolemmal, SSM, and interfibrillar, IFM, mitochondria due to their location within the cell. The SSM mitochondria are located just beneath the cell periphery and are more variable in shape than the IFM ranging up to $4 \mu \mathrm{m}$ in size. The IFM are densely packed between the myofibrils within the contractile apparatus and are smaller but more elongated ranging up to $2 \mu \mathrm{m}$ in length $(101,132)$. Below is a representative figure of where the mitochondria are located within the cell (Figure 1.3). 


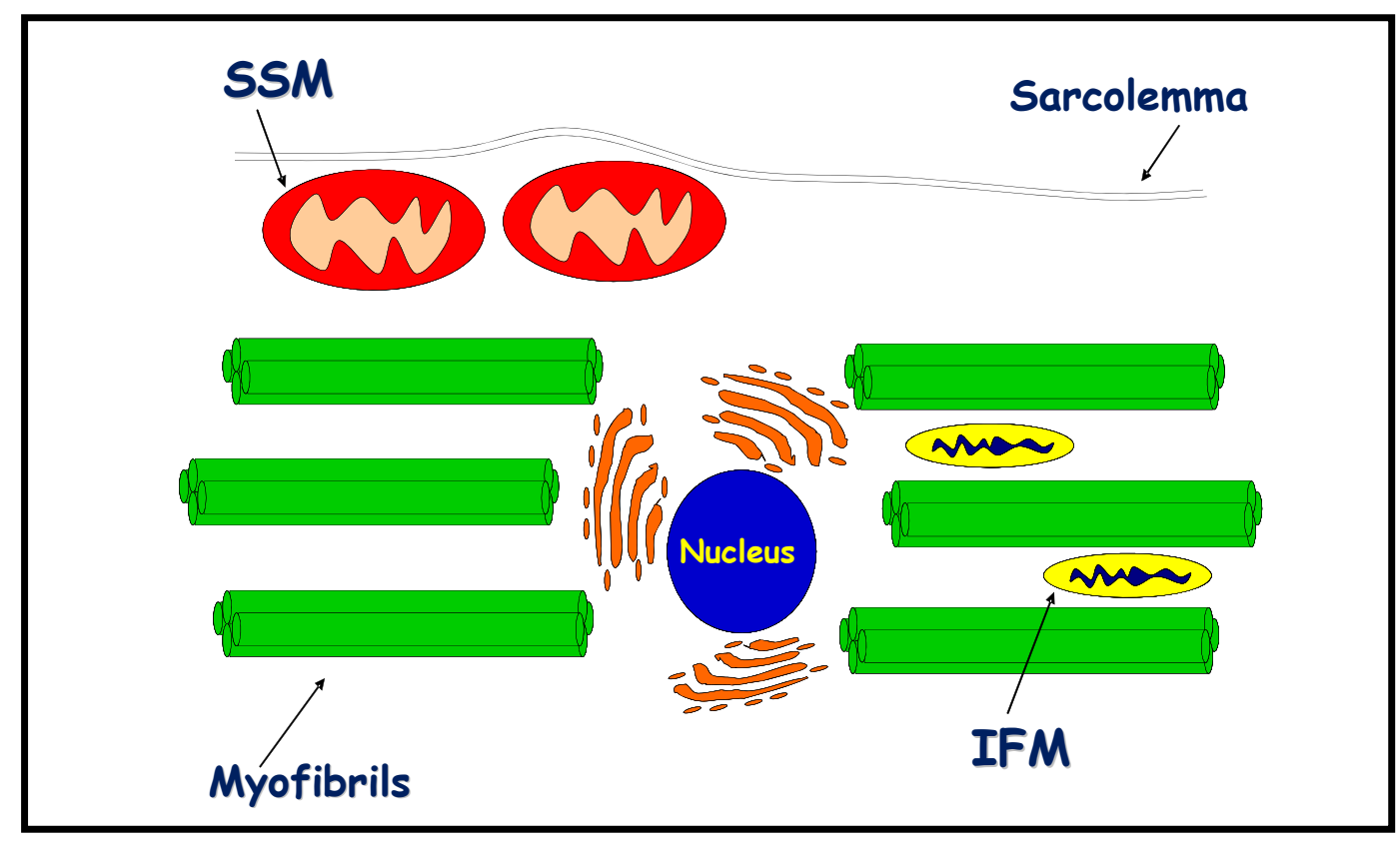

Figure 1.3 Mitochondrial subpopulations.

Through a series of centrifugations and protease digestion, Palmer et al. isolated and characterized these two subpopulations of mitochondria from rat cardiac muscle in 1977 (101). They determined that specific activities of succinate dehydrogenase and citrate synthase were higher in the IFM as compared to SSM and that IFM oxidized all substrates 1.5 times faster than the SSM. Later studies established that the SSM possess mainly lamelliform cristae whereas the IFM are mainly tubular or a mixture of tubular (rather than flat) and lamelliform cristae (115). This is important because it is suggested with the tubular cristae, there is a decrease in intracristal space which leads to a higher concentration of protons within these structures, thus increasing ATP synthase activity which enables oxidative phosphorylation (115). This is in agreement with the fact that the IFM have 1.5 fold higher oxidative rates and 3 fold higher ATP production. It has been suggested that the SSM produce ATP primarily for active transport of electrolytes and metabolites through sarcolemma, while the IFM produce ATP for contractile purposes primarily due to their location within the cell (101). In skeletal muscle, protein import rates differ between subpopulations, suggesting import of malate dehydrogenase was 3-4 fold higher in the IFM even though Grp75, an import protein, was lower in protein expression (137). It is suggested that import rate is higher in the IFM due to the higher respiratory rates, higher membrane potential and elevated 
ATP synthesis. Adhihetty et al. has also suggested that in skeletal muscle, IFM have a greater propensity for oxidant induced apoptosis (1). However, in heart, Palmer et al. suggested the capacity for calcium uptake in SSM was less than that of IFM, leading to release of cytochrome C from only SSM (102). This release is known to involve the PTP and occurs prior to apoptosis (75). More recently, it has been observed that the SSM are significantly more oxidized and have higher oxygen tension at rest due to their closer proximity to the capillaries in skeletal muscle (124).

Many have observed and suggested that these two spatially-distinct subpopulations respond differently with various physiological and pathological stimuli including ageing, $I / R$, exercise, caloric restriction, high fat feeding and heart failure $(1$, $20,27,28,37,66,71,73,77,78,114)$. With ischemia, it has been suggested that injury to both SSM and IFM takes place. However, ischemia in rabbit heart resulted in decreased oxidative phosphorylation (through cytochrome c oxidase) affects only the SSM (80). Further, the longer duration of ischemia, the greater damage to cardiolipin in the SSM. These two subpopulations have also been shown to be differentially affected with various cardiomyopathies and heart failure (79). Hoppel et al. observed decreased oxidative phosphorylation ( $50 \%$ reduction) specific to IFM in hearts from hamsters that develop cardiomyopathy at four months of age (59). Utilizing a dog model of heart failure, Rosca et al. suggested that both SSM and IFM had a 50\% reduction in oxidative phosphorylation along with decreased ETC activity (118). With a high fat diet and heart failure, there is an upregulation in enzymes involved with fatty acid oxidation in only SSM and an increase in state 3 respiration with fatty acid substrates such as palmitoyl carnitine (118). King et al. (73) reported significantly diminished state 3 and state 4 respiration in both SSM and IFM from STZ-induced rat heart which is in contrast to our previous study suggesting only the IFM are affected (28). Though aging is not necessarily a "disease", it does have profound effects on mitochondria. In particular, mitochondrial subpopulation response with aging has been studied extensively. With aging, it has been suggested that only the IFM have decreased ETC activities, particularly at complex III, and elevated Hydrogen peroxide formation accompanied with enhanced oxidative damage $(58,66,77)$. These results indicate that mitochondria 
located in different parts of the cell respond differently with various pathological insults, highlighting the importance of taking into account spatial location when evaluating mitochondrial dysfunction in cardiac and skeletal muscle.

\subsection{Inner Mitochondrial Membrane (IMM)}

The inner mitochondrial membrane (IMM) is particularly susceptible to ROSinduced oxidative damage because it is the major source of ROS production. We and others have shown that protein constituents and lipids within the IMM seem to be at risk with both type 1 and type 2 diabetic-associated dysfunction.

\section{6a Mitochondrial ROS production}

The ETC is the main site of superoxide generation through electron leakage as substrates passing through the ETC (134). ROS include the superoxide radical, $\mathrm{O}_{2}{ }^{-{ }^{-}}$, hydrogen peroxide, $\mathrm{H}_{2} \mathrm{O}_{2}$, and the hydroxyl radical, $\mathrm{OH}$. At physiologic $\mathrm{pH}$, superoxide dismutates or is catalyzed by superoxide dismutase (SOD) to form $\mathrm{H}_{2} \mathrm{O}_{2}(41)$. Superoxide is able to generate the more reactive hydroxyl radical through a series of steps dependent on the presence of redox metals (ie. Iron, copper). This reaction is termed the fenton reaction. Iron-sulfur centered proteins including electron transport chain proteins and aconitase all can react with hydrogen peroxide and increase the formation of hydroxyradicals. Hydroxyradicals can readily react with lipids within the inner mitochondrial membrane and cause disruption of the IMM integrity.

Mitochondria are the major intracellular site of superoxide production (108). It has been suggested that complex I and III are the major sites of ROS production (108). It is important to note that mitochondrial ROS may be generated at other sites as well, such as a-ketoglutarate dehydrogenase, and aconitase where conversion of superoxide to the hydroxyl radical results in inactivation of the enzyme (45). Other sources of ROS beyond mitochondria do exist and are as follows; oxygen radicals from peroxisomal $\beta$ oxidation of fatty acids, NADPH oxidase, xanthine oxidase, arachidonic acid metabolism, and microsomal P-450 enzymes $(7,18)$. 


\section{6b Cardiolipin}

One phospholipid predominantly within the IMM that is absolutely essential for proper mitochondrial function is cardiolipin $(C L)$. In mammals, $C L$ is localized predominantly in the IMM (25\% heart IMM, 4-5\% heart OM) where it facilitate mitochondrial function through a variety of mechanism (30). CL contains three glycerol backbones and four acyl chains (Figure 1.4) resulting in a structure that plays a role in proper mitochondrial function. $\mathrm{CL}$ is unique among other phospholipids because its diphosphatidylglycerol structure combined with four acyl chains which make it dimeric and results in a conical structure (60).

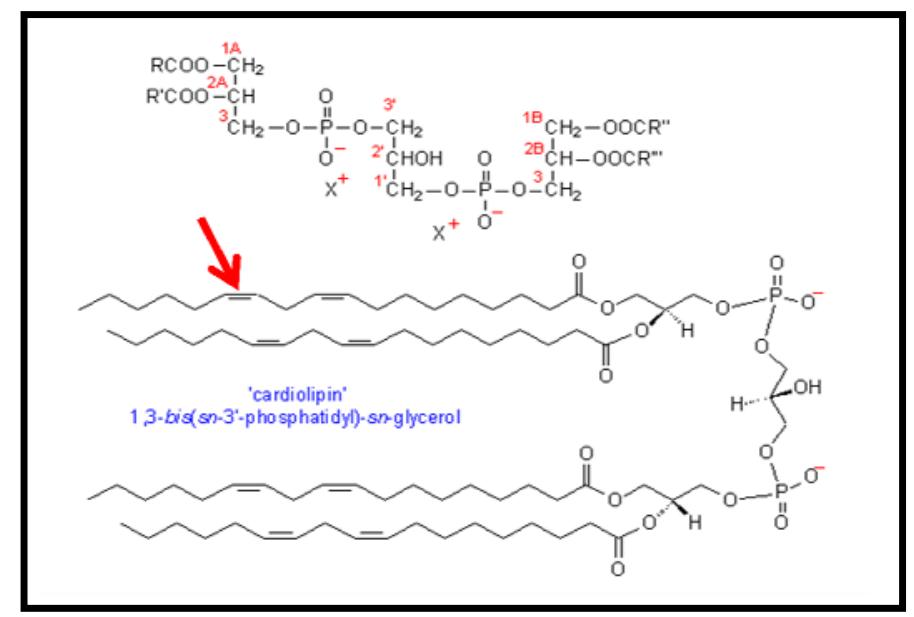

Figure 1.5 Cardiolipin Structure.

lipidlibrary.aocs.org/Lipids/dpg/index.htm

In mitochondria, $\mathrm{CL}$ in necessary for formation of contact sites between inner and outer membrane, stabilization of respiratory chain complexes and in the mitochondrial apoptotic signaling pathway (60). The abundance of unsaturated double bonds makes $\mathrm{CL}$ molecules more susceptible to ROS induced damage and this inturn, can precipitate further damage (60). The essential role of $C L$ in mitochondrial function and cardiac function has been understood through understanding genetic basis of barth syndrome. Barth syndrome patients have mutations in an X-linked gene, tafazzin, which induces alterations in $\mathrm{CL}$ metabolism. This inturn causes mitochondrial dysfunction and ultimately result in a dilated-cardiomyopathy. Tafazzin is the only enzyme that has been 
proven to be involved in $C L$ acyl chain remodeling in vivo $(53,121,146)$. $C L$ has been shown to interact with multiple IMM proteins including the ETC complexes that are involved in OXPHOS (120). CL is required for optimal activity of complex I (NADH ubiquinone oxidoreductase) (44), complex III (ubiquinone cytochrome c oxidoreductase) $(44,76)$, complex IV (cytochrome c oxidase) (125) and complex V (ATP synthase) (44). $\mathrm{CL}$ also allows the association and stabilization of "supercomplexes" (157). Supercomplex formation is thought to improve stabilization of respiratory chain complexes by abolishing the need for movement of substrates and products between complexes. $\mathrm{CL}$ is also required for proper activity and function of IMM carriers such as adenine nucleotide translocator, and carnitine-acylcarnitine, citrate, phosphate and pyruvate carriers (97). CL has also been suggested to play a role protein import into the mitochondria (60). However, it is not clearly understood how CL contributes to proper function of these carriers. It is thought that CL stabilizes the protein domain through a clamp-like manner with its two negatively charged head groups. Further, CL is suggested to function as a proton trap, restricting pumped protons within its polar head and subsequently providing structural basis for IMM potential and contributing protons for the ATP synthase (50).

Cardiolipin modulation has been observed in a number of pathologies including ischemia/reperfusion, aging and $\mathrm{DM}(22,52,103-106)$. These changes to $C L$ include: loss of CL content, changes in CL synthase activity, changes in acyl chain composition, and CL peroxidation. Han et al. (52) found depletion of the most predominant species of $\mathrm{CL}$ as well as remodeling of the remaining cardiolipin species in diabetic myocardium. Further, $\mathrm{CL}$ alterations occur as early as 5 days post STZ injection suggesting a potential contributor to the dysfunction observed in the type 1 diabetic heart including mitochondrial dysfunction (52).

\section{6c Mitochondrial Proteome}

Several studies have shown that mitochondrial proteomes seen to be preferentially altered in the diabetic heart $(16,51,67,131,140)$. Utilizing isotope labeling and two-dimensional gel electrophoresis, Turko et al. revealed up-regulation of 
fatty acid beta-oxidation proteins and down-regulation of several key ETC proteins. Further, they also examined decreases in VDAC-1, HSP60 and GRP75 in the STZinduced type 1 diabetic rat hearts (140). Utilizing the same model of type 1 DM (STZ), Hamblin et al. found that $12 / 24$ proteins in the LV identified as changing were mitochondrial proteins. Similar to Turko et al., FAO proteins were upregulated in the diabetic heart while TCA cycle and glycolytic proteins were down-regulated in the diabetic heart (51). In another model of type 1 DM (OVE26), Shen et al. revealed 12 out 20 proteins changing to be mitochondrial proteins. In contrast to the previous studies, majority of mitochondrial proteins, including ATP synthase subunits, were up regulated in the OVE26 heart (131). Using a label-free approach, Bugger et al. utilized the Akita mouse model of type $1 \mathrm{DM}$ and assessed proteomic alterations in mitochondria from kidney, heart, liver and brain (16). This study revealed that majority of proteins changing were in the liver, with little significant changes in the heart. These differences may be due to differences in the animal models that were used, differing proteomic approaches and shen et al. and Hamblin et al. used heart tissue while Turko et al. and Bugger et al. examined isolated mitochondria. Nevertheless, all of these studies examined modulation of the mitochondrial proteome with DM. Interestingly, no proteomic study has revealed changes in mitochondrial-encoded proteins, only nuclear encoded proteins. Downregulation of nuclear encoded mitochondrial proteins can be through several different mechanisms including: alterations in mitochondrial protein import, miRNA upregulation, transcriptional dysregulation or post-translational modifications that target nuclear-encoded proteins for degradation. Mitochondrial protein dysregulation can ultimately contribute to overall mitochondrial dysfunction, disruption of oxidative phosphorylation and other key mitochondrial processes. An oxidative environment can often lead to enhanced post-translational modification (PTM) to proteins including oxidations and deamidations (23). Though the contribution of deamidation and oxidation to protein dysfunction is somewhat unclear, these PTMs may alter protein structure/function making the protein more susceptible for degradation by the proteasome system $(65,116)$. Oxidative conditions have also been considered as a way through which proteins become more susceptible to deamidation. Though the underlying mechanism is not for certain, the evidence suggests that oxidative conditions 
may induce an increased flexibility of the polypeptide backbone or unfolding of the proteins, allowing for deamidation (23). Moreover, UV irradiation which causes increased ROS formation, lead to an increased protein deamidation in cultured cells (23)

\subsection{Antioxidant Defense}

Mitochondria have several mechanisms of defense against enhanced oxidative stress (47). One mechanism is uncoupling of the mitochondria which would reduce superoxide generation by decreasing mitochondrial membrane potential (135). However, this is achieved by decreasing oxidative phosphorylation and subsequent ATP production. In addition to this, there are multiple enzymatic reactions that decrease enhanced ROS generation. These enzymatic reactions include: dismutation of superoxide to $\mathrm{H}_{2} \mathrm{O}_{2}$ by manganese superoxide dismutase (MnSOD) and scavenging of $\mathrm{H}_{2} \mathrm{O}_{2}$ by catalase, glutathione peroxidases (GPX), or peroxiredoxins (47). Peroxiredoxins are a group of antioxidant enzymes that reduce hydrogen peroxide and alkyl hydroperoxides with reducing equivalents provided through the thioredoxin system. Peroxiredoxins can exist in the mitochondria, cytoplasm and peroxisome. Prdx3 and prdx5 are both found in the mitochondria (133).

\section{6a MnSOD}

MnSOD (SOD2) is also a mitochondrial targeted antioxidant that catalyzes the dismutation of superoxide into oxygen and hydrogen peroxide. The SOD-catalysed dismutation of superoxide may be written with the following half-reactions: 


$$
\begin{gathered}
M^{(n+1)+}-\mathrm{SOD}+\mathrm{O}_{2}^{-} \rightarrow \mathrm{M}^{\mathrm{n}+}-\mathrm{SOD}+\mathrm{O}_{2} \\
\mathrm{M}^{\mathrm{n}}-\mathrm{SOD}+\mathrm{O}_{2}^{-}+2 \mathrm{H}^{+} \rightarrow \mathrm{M}^{(\mathrm{n}+1)+}-\mathrm{SOD}+\mathrm{H}_{2} \mathrm{O}_{2}
\end{gathered}
$$

Figure 1.6 SOD-catalyzed dismutation of superoxide

Mice lacking SOD2 die several days after birth most likely due to enhanced oxidative stress (82). Mice lacking SOD1 develop a hepatocellular carcinoma, enhanced agerelated muscle mass loss, increased cataract formation and a decreased lifespan (34, 93). Knockout mice of any of the SOD enzyme are more susceptible to ROS-induced damage by oxidant supplementation (127). Shen et al. found that overexpression of MnSOD in the OVE26 model of type $1 \mathrm{DM}$ provided protection in the heart through improving mitochondrial respiration, mitochondrial mass and preserving cardiomyocyte contractility (130). However, overexpression of MnSOD did not completely restore respiratory capacity in mitochondria from the OVE26 hearts and overexpression altered expression of other antioxidant enzymes (130).

\section{6b Glutathione Peroxidases}

Glutathione peroxidases are enzymes that posses peroxidase activity whose main biological role is to protect the cell from oxidative stress and oxidative damage. There are several isozymes encoded by different genes, which vary in different cellular locations and have different substrates. Mammalian GPx1-4 are all selenoproteins, whereas GPx6 is a selenoprotein in humans with cysteine-containing homologues in rodents. GPx1-3 are homotetrameric proteins, and catalyze the reduction of hydrogen peroxide and organic hydroperoxides, whereas GPx4 has a monomeric structure and directly reduces phospholipid and cholesterol hydroperoxides. GPx4 is also involved in sperm maturation and male fertility due to its role in the sperm motility $(84,142)$. The presence of selenium is necessary for the antioxidant properties of glutathione 
peroxidase. The mechanism of action of these selenoproteins is at the selenocysteine site, which is in a Se (-) form as resting state. This is oxidized by the peroxide to $\mathrm{SeOH}$ which is then trapped by a GSH molecule to Se-SG and by another GSH molecule to Se (-) again, releasing a GS-SG by-product. Glutathione peroxidase 1 (GPx1) is the most abundant GPx and it is found in the cytoplasm and mitochondria of mammalian tissues, and the substrate is hydrogen peroxide (84).

An example reaction that glutathione peroxidase catalyzes is:

$$
2 \mathrm{GSH}+\mathrm{H}_{2} \mathrm{O}_{2} \rightarrow \mathrm{GS}-\mathrm{SG}+2 \mathrm{H}_{2} \mathrm{O}
$$

*GSH represents reduced monomeric glutathione

${ }^{*}$ GS-SG represents glutathione disulfide.

Glutathione reductase then reduces the oxidized glutathione to complete the cycle:

$$
\text { GS-SG + NADPH + } \mathrm{H}^{+} \rightarrow 2 \mathrm{GSH}+\mathrm{NADP}^{+}
$$

Figure 1.7 Glutathione peroxidase mechanism of action.

Transgenic mice overexpressing Gpx1 were more resistant to paraquat and other oxidant induced stressors (21). Though many studies suggest protection with increased levels of GPx1, one study suggested that transgenic overexpression of GPx1 lead to insulin resistance and obesity (89). These authors found that GPx1 tg mice developed hyperglycemia, hyperinsulinemia, and elevated plasma leptin levels. They also exhibited reduced phosphorylation of Akt after insulin stimulation. It was suggested that GPX1 activity may interrupt with insulin function and signaling by depleting ROS in the cytosol which is necessary for insulin signaling (89).

\section{6c Glutathione Peroxidase 4}

Glutathione peroxidase $4(\mathrm{GPx} 4)$, also referred to as phgpx, has a high preference for lipid hydroperoxides and is expressed in all cell types. It has several forms including mitochondrial, cytoplasmic and nuclear. However, GPx4 is found in 
much lower levels than other GPxs with the exception of being highly expressed in the testes. Glutathione peroxidase 4 is the only antioxidant that is capable of reducing lipid hydroperoxides to alcohols and to reduce free hydrogen peroxide to water $(84,143)$. Similar to other GPxs, the selenoalate anion is most likely oxidized by a hydroperoxide to selenenic acid and then reduced in its active state with two molecules of GSH, involving a selenadisulfide step. Normally, GSSG (oxidized GSH) is recycled by glutathione reductase (GR) at the expense of $\mathrm{NADPH} / \mathrm{H}+$ (Fig. 7A) (143). However, at low GSH levels (Fig. 7B), Gpx4 is the only Gpx that can convert into a protein thiol peroxidase and introduces disulfide bridges into proteins. If free thiols are no longer available, GPX4 may also become cross-linked through selenenylsulfide bridges ${ }^{*}$ ) or disulfide bridges to other proteins.

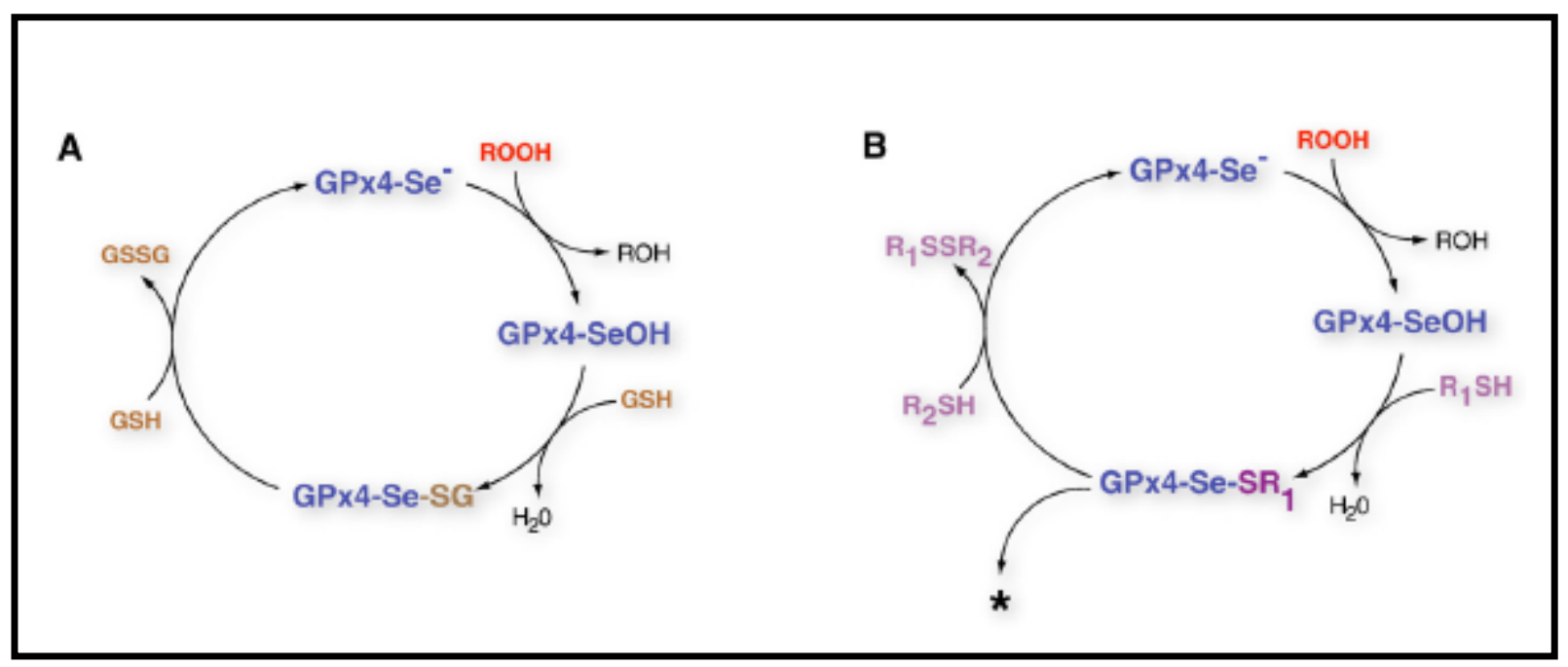

Figure 1.8 Glutathione peroxidase 4 catalysis.

As stated earlier, Gpx4 has three forms; mitochondrial, cytoplasmic and nuclear. Studies found that the nuclear form was expressed from its own promoter located upstream from the alternative exon $(86,92)$, where alternative transcription initiation at exon 1 determines if the longer (mitochondrial) form or the shorter (cytosolic) form is expressed. Many studies have shown that cytosolic Gpx4 is expressed in most tissues 
whereas the nuclear form is mostly in the testis (123). Further, the mitochondrial Gpx4 is mostly found in the testes as well (87). In converse to Gpx1, Gpx4-knockout mice die at embryonic stage (E7.5) indicating that Gpx4 is a very important and an essential selenoprotein $(64,153)$. A constitutive knockout of $\mathrm{Gpx} 4, \mathrm{Gpx} 4+/-$, suggested that lung fibroblasts with one Gpx4 allele were more susceptible to hydrogen peroxide, cadmium and cumene hydroperoxide (46). On the other hand, several transgenic models have been generated which overexpress Gpx4 including one model from our lab. Mice overexpressing human Gpx4 are more resistant to diquat-induced liver damage through preservation of ATP and diminished oxidative damage (109). Utilizing the same tg animal, Ran et al. also suggested that overexpression of Gpx4 provided protection against oxidative stress-induced apoptosis as well as attenuated plaque formation in ApoE deficient mice (49). Transgene expression was approximately 3 fold higher than the endogenous Gpx4 level, and it was expressed in multiple compartments including mitochondria, cytosol, nuclei and microsome. All of these reports in mice are in agreement with numerous cell culture studies showing Gpx4 protects cells against apoptosis, oxidants, DNA-damaging agents, and glucose depletion (25). In contrast, we have generated a transgenic mouse line in which Gpx4 is targeted specifically to the mitochondria and at a much higher expression relative to previous Gpx4 constructs (109), enabling us to determine the influence of mitochondrial-specific overexpression of this enzyme (29). Our lab has suggested that overexpression of mitochondrial Gpx4 (mPHGPx) preserves cardiac contractile and mitochondrial function following I/R (29). Transgenic overexpression of $\mathrm{mPHPGx}$ also attenuated accumulation of lipid peroxidation by-products and preserves mitochondrial function in both subpopulations of mitochondria following I/R (29). MPHGPx is an enzyme specific to the IMM, and it scavenges phospholipid hydroperoxides including cardiolipin hydroperoxide in an attempt to preserve mitochondrial inner membrane integrity $(9,57,98)$. Removal of cardiolipin from the mitochondria disrupts mitochondrial function emphasizing that it is absolutely essential for proper mitochondrial function. As stated earlier, cardiolipin is located predominantly within the IMM where it interacts with a large number of mitochondrial proteins. Interaction effects functional activation of enzymes, especially those within the ETC (72). Cardiolipin has been identified as an integral component of 
mitochondrial complex III, complex IV and the ADP-ATP-carrier (ANT), and it is known to be essential for the stability of the structure of ANT. Removal of cardiolipin breaks up the ETC complexes and decreases functionality of the IMM (72). Thus, the ability to preserve such a phospholipid within the IMM with overexpression of MPHGPx may be a critical component in providing mitochondrial protection. Hence, our goal is to elucidate a possible mechanism of protection with DM utilizing our mitochondrial specific PHGPX overexpressing mouse.

\subsection{Summary}

One in three Americans born after 2000 will develop DM in their lifetime. These statistics are shocking and implicate the necessity for possible diabetic therapeutic strategies. Mitochondrial dysfunction and an enhanced oxidative milieu seem to be underlying contributors to the pathogenesis of diabetic cardiomyopathy. Our ultimate goal is to determine whether mitochondria located in different parts of the cell are impacted differentially with DM in hopes of elucidating potential mechanisms involved in mitochondrial dysfunction. Further, mPHGPx transgenic overexpression should contribute to our understanding of the role played by this enzyme during type $1 \mathrm{DM}$, opening its door as a potential mitochondrially-driven therapeutic. 


\section{REFERENCES}

1. Adhihetty PJ, Ljubicic V, Menzies KJ, and Hood DA. Differential susceptibility of subsarcolemmal and intermyofibrillar mitochondria to apoptotic stimuli. Am J Physiol Cell Physiol 289: C994-C1001, 2005.

2. An D and Rodrigues $\mathbf{B}$. Role of changes in cardiac metabolism in development of diabetic cardiomyopathy. Am J Physiol Heart Circ Physiol 291: H1489-1506, 2006.

3. Asbun J, Manso AM, and Villarreal FJ. Profibrotic influence of high glucose concentration on cardiac fibroblast functions: effects of losartan and vitamin E. Am J Physiol Heart Circ Physiol 288: H227-234, 2005.

4. Ban CR and Twigg SM. Fibrosis in DM complications: pathogenic mechanisms and circulating and urinary markers. Vasc Health Risk Manag 4: 575-596, 2008.

5. Barouch LA, Gao D, Chen L, Miller KL, Xu W, Phan AC, Kittleson MM, Minhas KM, Berkowitz DE, Wei C, and Hare JM. Cardiac myocyte apoptosis is associated with increased DNA damage and decreased survival in murine models of obesity. Circ Res 98: 119-124, 2006.

6. Bauters C, Lamblin N, Mc Fadden EP, Van Belle E, Millaire A, and de Groote P. Influence of DM mellitus on heart failure risk and outcome. Cardiovasc Diabetol 2: 1, 2003.

7. Beckman KB and Ames BN. The free radical theory of aging matures. Physiol Rev 78: 547-581, 1998.

8. Belke DD, Larsen TS, Gibbs EM, and Severson DL. Altered metabolism causes cardiac dysfunction in perfused hearts from diabetic $(\mathrm{db} / \mathrm{db})$ mice. Am J Physiol Endocrinol Metab 279: E1104-1113, 2000.

9. Borchert A, Wang CC, Ufer C, Schiebel H, Savaskan NE, and Kuhn H. The role of phospholipid hydroperoxide glutathione peroxidase isoforms in murine embryogenesis. J Biol Chem 281: 19655-19664, 2006.

10. Boudina S and Abel ED. Diabetic cardiomyopathy, causes and effects. Rev Endocr Metab Disord 11: 31-39, 2010. 
11. Boudina S, Sena S, Theobald H, Sheng X, Wright JJ, Hu XX, Aziz S, Johnson JI, Bugger H, Zaha VG, and Abel ED. Mitochondrial energetics in the heart in obesity-related DM: direct evidence for increased uncoupled respiration and activation of uncoupling proteins. DM 56: 2457-2466, 2007.

12. Brooks BA, Franjic B, Ban CR, Swaraj K, Yue DK, Celermajer DS, and Twigg SM. Diastolic dysfunction and abnormalities of the microcirculation in type 2 DM. DM Obes Metab 10: 739-746, 2008.

13. Buchanan J, Mazumder PK, Hu P, Chakrabarti G, Roberts MW, Yun UJ, Cooksey RC, Litwin SE, and Abel ED. Reduced cardiac efficiency and altered substrate metabolism precedes the onset of hyperglycemia and contractile dysfunction in two mouse models of insulin resistance and obesity. Endocrinology 146: 5341-5349, 2005.

14. Bugger H and Abel ED. Rodent models of diabetic cardiomyopathy. Dis Model Mech 2: 454-466, 2009.

15. Bugger H, Boudina S, Hu XX, Tuinei J, Zaha VG, Theobald HA, Yun UJ, McQueen AP, Wayment B, Litwin SE, and Abel ED. Type 1 diabetic akita mouse hearts are insulin sensitive but manifest structurally abnormal mitochondria that remain coupled despite increased uncoupling protein 3. DM 57: 2924-2932, 2008.

16. Bugger H, Chen D, Riehle C, Soto J, Theobald HA, Hu XX, Ganesan B, Weimer BC, and Abel ED. Tissue-specific remodeling of the mitochondrial proteome in type 1 diabetic akita mice. DM 58: 1986-1997, 2009.

17. Cai L, Li W, Wang G, Guo L, Jiang Y, and Kang YJ. Hyperglycemia-induced apoptosis in mouse myocardium: mitochondrial cytochrome C-mediated caspase-3 activation pathway. DM 51: 1938-1948, 2002.

18. Ceriello A. New insights on oxidative stress and diabetic complications may lead to a "causal" antioxidant therapy. DM Care 26: 1589-1596, 2003.

19. Chao TT, lanuzzo CD, Armstrong RB, Albright JT, and Anapolle SE. Ultrastructural alterations in skeletal muscle fibers of streptozotocin-diabetic rats. Cell Tissue Res 168: 239-246, 1976. 
20. Chen Q, Moghaddas S, Hoppel CL, and Lesnefsky EJ. Ischemic defects in the electron transport chain increase the production of reactive oxygen species from isolated rat heart mitochondria. American journal of physiology 294: C460-466, 2008.

21. Cheng W, Fu YX, Porres JM, Ross DA, and Lei XG. Selenium-dependent cellular glutathione peroxidase protects mice against a pro-oxidant-induced oxidation of NADPH, NADH, lipids, and protein. FASEB J 13: 1467-1475, 1999.

22. Chicco AJ and Sparagna GC. Role of cardiolipin alterations in mitochondrial dysfunction and disease. Am J Physiol Cell Physiol 292: C33-44, 2007.

23. Cimmino A, Capasso R, Muller F, Sambri I, Masella L, Raimo M, De Bonis ML, D'Angelo S, Zappia V, Galletti P, and Ingrosso D. Protein isoaspartate methyltransferase prevents apoptosis induced by oxidative stress in endothelial cells: role of $\mathrm{Bcl}-\mathrm{XI}$ deamidation and methylation. PLoS One 3: e3258, 2008.

24. Coleman DL. Obese and DM: two mutant genes causing DM-obesity syndromes in mice. Diabetologia 14: 141-148, 1978.

25. Conrad M, Schneider M, Seiler A, and Bornkamm GW. Physiological role of phospholipid hydroperoxide glutathione peroxidase in mammals. Biol Chem 388: 10191025, 2007.

26. Cotran Ra. Pathological Basis of Disease.

27. Dabkowski ER, Baseler WA, Williamson CL, Powell M, Razunguzwa TT, Frisbee JC, and Hollander JM. Mitochondrial Dysfunction in the Type 2 Diabetic Heart is Associated with Alterations in Spatially-Distinct Mitochondrial Proteomes. Am J Physiol Heart Circ Physiol, 2010.

28. Dabkowski ER, Williamson CL, Bukowski VC, Chapman RS, Leonard SS, Peer CJ, Callery PS, and Hollander JM. Diabetic cardiomyopathy-associated dysfunction in spatially distinct mitochondrial subpopulations. Am J Physiol Heart Circ Physiol 296: H359-369, 2009.

29. Dabkowski ER, Williamson CL, and Hollander JM. Mitochondria-specific transgenic overexpression of phospholipid hydroperoxide glutathione peroxidase (GPx4) attenuates ischemia/reperfusion-associated cardiac dysfunction. Free radical biology \& medicine 45: 855-865, 2008. 
30. de Kroon Al, Dolis D, Mayer A, Lill R, and de Kruijff B. Phospholipid composition of highly purified mitochondrial outer membranes of rat liver and Neurospora crassa. Is cardiolipin present in the mitochondrial outer membrane? Biochim Biophys Acta 1325: 108-116, 1997.

31. Dong F, Zhang X, Yang X, Esberg LB, Yang H, Zhang Z, Culver B, and Ren J. Impaired cardiac contractile function in ventricular myocytes from leptin-deficient ob/ob obese mice. J Endocrinol 188: 25-36, 2006.

32. Du Y, Miller CM, and Kern TS. Hyperglycemia increases mitochondrial superoxide in retina and retinal cells. Free Radic Biol Med 35: 1491-1499, 2003.

33. Duan J, Zhang HY, Adkins SD, Ren BH, Norby FL, Zhang X, Benoit JN, Epstein PN, and Ren J. Impaired cardiac function and IGF-I response in myocytes from calmodulin-diabetic mice: role of Akt and RhoA. Am J Physiol Endocrinol Metab 284: E366-376, 2003.

34. Elchuri S, Oberley TD, Qi W, Eisenstein RS, Jackson Roberts L, Van Remmen H, Epstein CJ, and Huang TT. CuZnSOD deficiency leads to persistent and widespread oxidative damage and hepatocarcinogenesis later in life. Oncogene 24: 367-380, 2005.

35. Epstein PN, Overbeek PA, and Means AR. Calmodulin-induced early-onset DM in transgenic mice. Cell 58: 1067-1073, 1989.

36. Fang ZY, Prins JB, and Marwick TH. Diabetic cardiomyopathy: evidence, mechanisms, and therapeutic implications. Endocr Rev 25: 543-567, 2004.

37. Fannin SW, Lesnefsky EJ, Slabe TJ, Hassan MO, and Hoppel CL. Aging selectively decreases oxidative capacity in rat heart interfibrillar mitochondria. Archives of biochemistry and biophysics 372: 399-407, 1999.

38. Fein FS and Sonnenblick EH. Diabetic cardiomyopathy. Cardiovasc Drugs Ther 8: 65-73, 1994.

39. Ferko M, Gvozdjakova A, Kucharska J, Mujkosova J, Waczulikova I, Styk J, Ravingerova T, Ziegelhoffer-Mihalovicova B, and Ziegelhoffer A. Functional remodeling of heart mitochondria in acute DM: interrelationships between damage, endogenous protection and adaptation. Gen Physiol Biophys 25: 397-413, 2006. 
40. Flarsheim CE, Grupp IL, and Matlib MA. Mitochondrial dysfunction accompanies diastolic dysfunction in diabetic rat heart. The American journal of physiology 271: H192-202, 1996.

41. Fridovich I. Superoxide anion radical (O2-.), superoxide dismutases, and related matters. J Biol Chem 272: 18515-18517, 1997.

42. Friedman JM and Halaas JL. Leptin and the regulation of body weight in mammals. Nature 395: 763-770, 1998.

43. Frustaci A, Kajstura J, Chimenti C, Jakoniuk I, Leri A, Maseri A, NadalGinard B, and Anversa P. Myocardial cell death in human DM. Circ Res 87: 11231132, 2000.

44. Fry $\mathbf{M}$ and Green DE. Cardiolipin requirement for electron transfer in complex I and III of the mitochondrial respiratory chain. J Biol Chem 256: 1874-1880, 1981.

45. Gardner PR. Superoxide-driven aconitase FE-S center cycling. Biosci Rep 17: 33-42, 1997.

46. Garry MR, Kavanagh TJ, Faustman EM, Sidhu JS, Liao R, Ware C, Vliet PA, and Deeb SS. Sensitivity of mouse lung fibroblasts heterozygous for GPx4 to oxidative stress. Free Radic Biol Med 44: 1075-1087, 2008.

47. Green K, Brand MD, and Murphy MP. Prevention of mitochondrial oxidative damage as a therapeutic strategy in DM. DM 53 Suppl 1: S110-118, 2004.

48. Gross MD, Harris S, and Beyer RE. The effect of streptozotocin-induced DM on oxidative phosphorylation and related reactions in skeletal muscle mitochondria. Horm Metab Res 4: 1-7, 1972.

49. Guo Z, Ran Q, Roberts LJ, 2nd, Zhou L, Richardson A, Sharan C, Wu D, and Yang H. Suppression of atherogenesis by overexpression of glutathione peroxidase-4 in apolipoprotein E-deficient mice. Free Radic Biol Med 44: 343-352, 2008.

50. Haines TH and Dencher NA. Cardiolipin: a proton trap for oxidative phosphorylation. FEBS Lett 528: 35-39, 2002.

51. Hamblin M, Friedman DB, Hill S, Caprioli RM, Smith HM, and Hill MF. Alterations in the diabetic myocardial proteome coupled with increased myocardial oxidative stress underlies diabetic cardiomyopathy. J Mol Cell Cardiol 42: 884-895, 2007. 
52. Han X, Yang J, Yang K, Zhao Z, Abendschein DR, and Gross RW. Alterations in myocardial cardiolipin content and composition occur at the very earliest stages of DM: a shotgun lipidomics study. Biochemistry 46: 6417-6428, 2007.

53. Hauff KD and Hatch GM. Cardiolipin metabolism and Barth Syndrome. Prog Lipid Res 45: 91-101, 2006.

54. Herlein JA, Fink BD, O'Malley Y, and Sivitz WI. Superoxide and respiratory coupling in mitochondria of insulin-deficient diabetic rats. Endocrinology 150: 46-55, 2009.

55. Hoeldtke RD, Bryner KD, McNeill DR, Warehime SS, Van Dyke K, and Hobbs G. Oxidative stress and insulin requirements in patients with recent-onset type 1 DM. J Clin Endocrinol Metab 88: 1624-1628, 2003.

56. Hojlund K, Wrzesinski K, Larsen PM, Fey SJ, Roepstorff P, Handberg A, Dela F, Vinten J, McCormack JG, Reynet C, and Beck-Nielsen H. Proteome analysis reveals phosphorylation of ATP synthase beta -subunit in human skeletal muscle and proteins with potential roles in type 2 DM. J Biol Chem 278: 10436-10442, 2003.

57. Hollander JM, Lin KM, Scott BT, and Dillmann WH. Overexpression of PHGPX and HSP60/10 protects against ischemia/reoxygenation injury. Free radical biology \& medicine 35: 742-751, 2003.

58. Hoppel CL, Moghaddas S, and Lesnefsky EJ. Interfibrillar cardiac mitochondrial comples III defects in the aging rat heart. Biogerontology 3: 41-44, 2002.

59. Hoppel CL, Tandler B, Parland W, Turkaly JS, and Albers LD. Hamster cardiomyopathy. A defect in oxidative phosphorylation in the cardiac interfibrillar mitochondria. J Biol Chem 257: 1540-1548, 1982.

60. Houtkooper RH and Vaz FM. Cardiolipin, the heart of mitochondrial metabolism. Cell Mol Life Sci 65: 2493-2506, 2008.

61. How OJ, Aasum E, Severson DL, Chan WY, Essop MF, and Larsen TS. Increased myocardial oxygen consumption reduces cardiac efficiency in diabetic mice. DM 55: 466-473, 2006.

62. Hsueh W, Abel ED, Breslow JL, Maeda N, Davis RC, Fisher EA, Dansky H, McClain DA, Mclndoe R, Wassef MK, Rabadan-Diehl C, and Goldberg IJ. Recipes 
for creating animal models of diabetic cardiovascular disease. Circ Res 100: 1415-1427, 2007.

63. Hummel M, Bonifacio E, Schmid S, Walter M, Knopff A, and Ziegler AG. Brief communication: early appearance of islet autoantibodies predicts childhood type 1 DM in offspring of diabetic parents. Ann Intern Med 140: 882-886, 2004.

64. Imai $\mathbf{H}$ and Nakagawa $\mathbf{Y}$. Biological significance of phospholipid hydroperoxide glutathione peroxidase (PHGPx, GPx4) in mammalian cells. Free Radic Biol Med 34: 145-169, 2003.

65. Iwai K, Drake SK, Wehr NB, Weissman AM, LaVaute T, Minato N, Klausner RD, Levine RL, and Rouault TA. Iron-dependent oxidation, ubiquitination, and degradation of iron regulatory protein 2: implications for degradation of oxidized proteins. Proc Natl Acad Sci U S A 95: 4924-4928, 1998.

66. Judge S, Jang YM, Smith A, Hagen T, and Leeuwenburgh C. Age-associated increases in oxidative stress and antioxidant enzyme activities in cardiac interfibrillar mitochondria: implications for the mitochondrial theory of aging. Faseb $\mathrm{J}$ 19: 419-421, 2005.

67. Jüllig M, Hickey AJ, Middleditch MJ, Crossman DJ, Lee SC, and Cooper GJS. Characterization of proteomic changes in cardiac mitochondria in streptozotocindiabetic rats using iTRAQ $<$ SUP $><$ FONT SIZE $='-1$ ' $>$ TM $</ F O N T></ S U P>$ isobaric tags. PROTEOMICS - Clinical Applications 1: 565-576, 2007.

68. Kajstura J, Fiordaliso F, Andreoli AM, Li B, Chimenti S, Medow MS, Limana F, Nadal-Ginard B, Leri A, and Anversa P. IGF-1 overexpression inhibits the development of diabetic cardiomyopathy and angiotensin II-mediated oxidative stress. DM 50: 1414-1424, 2001.

69. Kannel WB and McGee DL. DM and cardiovascular disease. The Framingham study. Jama 241: 2035-2038, 1979.

70. Karakelides H, Asmann YW, Bigelow ML, Short KR, Dhatariya K, CoenenSchimke J, Kahl J, Mukhopadhyay D, and Nair KS. Effect of insulin deprivation on muscle mitochondrial ATP production and gene transcript levels in type 1 diabetic subjects. DM 56: 2683-2689, 2007. 
71. Kelley DE, He J, Menshikova EV, and Ritov VB. Dysfunction of mitochondria in human skeletal muscle in type 2 DM. DM 51: 2944-2950, 2002.

72. Kiebish MA, Han X, Cheng H, Chuang JH, and Seyfried TN. Cardiolipin and electron transport chain abnormalities in mouse brain tumor mitochondria: lipidomic evidence supporting the Warburg theory of cancer. Journal of lipid research 49: 25452556, 2008.

73. King KL, Young ME, Kerner J, Huang H, O'Shea KM, Alexson SE, Hoppel CL, and Stanley WC. DM or peroxisome proliferator-activated receptor alpha agonist increases mitochondrial thioesterase I activity in heart. Journal of lipid research 48: 1511-1517, 2007.

74. Koves TR, Ussher JR, Noland RC, Slentz D, Mosedale M, Ilkayeva O, Bain J, Stevens R, Dyck JR, Newgard CB, Lopaschuk GD, and Muoio DM. Mitochondrial overload and incomplete fatty acid oxidation contribute to skeletal muscle insulin resistance. Cell Metab 7: 45-56, 2008.

75. Kroemer G. The mitochondrial permeability transition pore complex as a pharmacological target. An introduction. Curr Med Chem 10: 1469-1472, 2003.

76. Lange C, Nett JH, Trumpower BL, and Hunte C. Specific roles of proteinphospholipid interactions in the yeast cytochrome bc1 complex structure. EMBO J 20: 6591-6600, 2001.

77. Leeuwenburgh C, Gurley CM, Strotman BA, and Dupont-Versteegden EE. Age-related differences in apoptosis with disuse atrophy in soleus muscle. Am J Physiol Regul Integr Comp Physiol 288: R1288-1296, 2005.

78. Lesnefsky EJ, Chen Q, Slabe TJ, Stoll MS, Minkler PE, Hassan MO, Tandler B, and Hoppel CL. Ischemia, rather than reperfusion, inhibits respiration through cytochrome oxidase in the isolated, perfused rabbit heart: role of cardiolipin. Am J Physiol Heart Circ Physiol 287: H258-267, 2004.

79. Lesnefsky EJ, Moghaddas S, Tandler B, Kerner J, and Hoppel CL. Mitochondrial dysfunction in cardiac disease: ischemia--reperfusion, aging, and heart failure. J Mol Cell Cardiol 33: 1065-1089, 2001. 
80. Lesnefsky EJ, Tandler B, Ye J, Slabe TJ, Turkaly J, and Hoppel CL. Myocardial ischemia decreases oxidative phosphorylation through cytochrome oxidase in subsarcolemmal mitochondria. Am J Physiol 273: H1544-1554, 1997.

81. Li $\mathbf{L}$ and Renier G. Activation of nicotinamide adenine dinucleotide phosphate (reduced form) oxidase by advanced glycation end products links oxidative stress to altered retinal vascular endothelial growth factor expression. Metabolism 55: 15161523, 2006.

82. Li Y, Huang TT, Carlson EJ, Melov S, Ursell PC, Olson JL, Noble LJ, Yoshimura MP, Berger C, Chan PH, Wallace DC, and Epstein CJ. Dilated cardiomyopathy and neonatal lethality in mutant mice lacking manganese superoxide dismutase. Nat Genet 11: 376-381, 1995.

83. Liang Q, Carlson EC, Donthi RV, Kralik PM, Shen X, and Epstein PN. Overexpression of metallothionein reduces diabetic cardiomyopathy. DM 51: 174-181, 2002.

84. Lu J and Holmgren A. Selenoproteins. J Biol Chem 284: 723-727, 2009.

85. Lu Z, Jiang YP, Xu XH, Ballou LM, Cohen IS, and Lin RZ. Decreased L-type Ca2+ current in cardiac myocytes of type 1 diabetic Akita mice due to reduced phosphatidylinositol 3-kinase signaling. DM 56: 2780-2789, 2007.

86. Maiorino M, Scapin M, Ursini F, Biasolo M, Bosello V, and Flohe L. Distinct promoters determine alternative transcription of gpx-4 into phospholipid-hydroperoxide glutathione peroxidase variants. J Biol Chem 278: 34286-34290, 2003.

87. Marinari UM, Monacelli R, Cottalasso D, and Novelli A. Effects of alloxan DM and insulin on morphology and certain functional activities of mitochondria of the rat liver and heart. Acta Diabetol Lat 11: 296-314, 1974.

88. Marra G, Cotroneo P, Pitocco D, Manto A, Di Leo MA, Ruotolo V, Caputo S, Giardina B, Ghirlanda G, and Santini SA. Early increase of oxidative stress and reduced antioxidant defenses in patients with uncomplicated type $1 \mathrm{DM}$ : a case for gender difference. DM Care 25: 370-375, 2002.

89. McClung JP, Roneker CA, Mu W, Lisk DJ, Langlais P, Liu F, and Lei XG. Development of insulin resistance and obesity in mice overexpressing cellular glutathione peroxidase. Proc Natl Acad Sci U S A 101: 8852-8857, 2004. 
90. Mootha VK, Lindgren CM, Eriksson KF, Subramanian A, Sihag S, Lehar J, Puigserver P, Carlsson E, Ridderstrale M, Laurila E, Houstis N, Daly MJ, Patterson N, Mesirov JP, Golub TR, Tamayo P, Spiegelman B, Lander ES, Hirschhorn JN, Altshuler D, and Groop LC. PGC-1alpha-responsive genes involved in oxidative phosphorylation are coordinately downregulated in human DM. Nat Genet 34: 267-273, 2003.

91. Moreira PI, Rolo AP, Sena C, Seica R, Oliveira CR, and Santos MS. Insulin attenuates DM-related mitochondrial alterations: a comparative study. Med Chem 2: 299-308, 2006.

92. Moreno SG, Laux G, Brielmeier M, Bornkamm GW, and Conrad M. Testisspecific expression of the nuclear form of phospholipid hydroperoxide glutathione peroxidase (PHGPx). Biol Chem 384: 635-643, 2003.

93. Muller FL, Song W, Liu Y, Chaudhuri A, Pieke-Dahl S, Strong R, Huang TT, Epstein CJ, Roberts LJ, 2nd, Csete M, Faulkner JA, and Van Remmen H. Absence of CuZn superoxide dismutase leads to elevated oxidative stress and acceleration of age-dependent skeletal muscle atrophy. Free Radic Biol Med 40: 1993-2004, 2006.

94. Nielsen J, Mogensen M, Vind BF, Sahlin K, Hojlund K, Schroder HD, and Ortenblad N. Increased subsarcolemmal lipids in type $2 \mathrm{DM}$ : effect of training on localization of lipids, mitochondria, and glycogen in sedentary human skeletal muscle. Am J Physiol Endocrinol Metab 298: E706-713, 2010.

95. Nielsen LB, Bartels ED, and Bollano E. Overexpression of apolipoprotein B in the heart impedes cardiac triglyceride accumulation and development of cardiac dysfunction in diabetic mice. J Biol Chem 277: 27014-27020, 2002.

96. Nishikawa T, Edelstein D, Du XL, Yamagishi S, Matsumura T, Kaneda $Y$, Yorek MA, Beebe D, Oates PJ, Hammes HP, Giardino I, and Brownlee M. Normalizing mitochondrial superoxide production blocks three pathways of hyperglycaemic damage. Nature 404: 787-790, 2000.

97. Noel H and Pande SV. An essential requirement of cardiolipin for mitochondrial carnitine acylcarnitine translocase activity. Lipid requirement of carnitine acylcarnitine translocase. Eur J Biochem 155: 99-102, 1986. 
98. Nomura K, Imai H, Koumura T, Kobayashi T, and Nakagawa Y. Mitochondrial phospholipid hydroperoxide glutathione peroxidase inhibits the release of cytochrome $\mathrm{c}$ from mitochondria by suppressing the peroxidation of cardiolipin in hypoglycaemiainduced apoptosis. Biochem J 351: 183-193, 2000.

99. Oliveira PJ, Seica R, Coxito PM, Rolo AP, Palmeira CM, Santos MS, and Moreno AJ. Enhanced permeability transition explains the reduced calcium uptake in cardiac mitochondria from streptozotocin-induced diabetic rats. FEBS Lett 554: 511514, 2003.

100. Ozasa N, Furukawa Y, Morimoto T, Tadamura E, Kita T, and Kimura T. Relation among left ventricular mass, insulin resistance, and hemodynamic parameters in type 2 DM. Hypertens Res 31: 425-432, 2008.

101. Palmer JW, Tandler B, and Hoppel CL. Biochemical properties of subsarcolemmal and interfibrillar mitochondria isolated from rat cardiac muscle. The Journal of biological chemistry 252: 8731-8739, 1977.

102. Palmer JW, Tandler B, and Hoppel CL. Heterogeneous response of subsarcolemmal heart mitochondria to calcium. Am J Physiol 250: H741-748, 1986.

103. Paradies G, Petrosillo G, Pistolese M, Di Venosa N, Serena D, and Ruggiero FM. Lipid peroxidation and alterations to oxidative metabolism in mitochondria isolated from rat heart subjected to ischemia and reperfusion. Free Radic Biol Med 27: 42-50, 1999.

104. Paradies G, Ruggiero FM, Dinoi P, Petrosillo G, and Quagliariello E. Decreased cytochrome oxidase activity and changes in phospholipids in heart mitochondria from hypothyroid rats. Arch Biochem Biophys 307: 91-95, 1993.

105. Paradies G, Ruggiero FM, Petrosillo G, and Quagliariello E. Age-dependent decrease in the cytochrome c oxidase activity and changes in phospholipids in rat-heart mitochondria. Arch Gerontol Geriatr 16: 263-272, 1993.

106. Paradies G, Ruggiero FM, Petrosillo G, and Quagliariello E. Enhanced cytochrome oxidase activity and modification of lipids in heart mitochondria from hyperthyroid rats. Biochim Biophys Acta 1225: 165-170, 1994. 
107. Rabol R, Boushel R, and Dela F. Mitochondrial oxidative function and type 2 DM. Applied physiology, nutrition, and metabolism = Physiologie appliquee, nutrition et metabolisme 31: 675-683, 2006.

108. Raha S and Robinson BH. Mitochondria, oxygen free radicals, disease and ageing. Trends Biochem Sci 25: 502-508, 2000.

109. Ran Q, Liang H, Gu M, Qi W, Walter CA, Roberts LJ, 2nd, Herman B, Richardson A, and Van Remmen H. Transgenic mice overexpressing glutathione peroxidase 4 are protected against oxidative stress-induced apoptosis. J Biol Chem 279: 55137-55146, 2004.

110. Razak F and Anand SS. Impaired mitochondrial activity in the insulin-resistant offspring of patients with type 2 DM. Petersen KF, Dufour S, Befroy D, Garcia R, Shulman GI. N Engl J Med 2004; 350: 664-71. Vasc Med 9: 223-224, 2004.

111. Regan TJ and Lyons MM. Diabetic cardiomyopathy. Circulation 83: 1830, 1991. 112. Regan TJ, Lyons MM, Ahmed SS, Levinson GE, Oldewurtel HA, Ahmad MR, and Haider B. Evidence for cardiomyopathy in familial DM mellitus. J Clin Invest 60: 884-899, 1977.

113. Research MFfMEa. Diabetic Ketoacidosis, 2006.

114. Ritov VB, Menshikova EV, He J, Ferrell RE, Goodpaster BH, and Kelley DE. Deficiency of subsarcolemmal mitochondria in obesity and type 2 DM. DM 54: 8-14, 2005.

115. Riva A, Tandler B, Loffredo F, Vazquez E, and Hoppel C. Structural differences in two biochemically defined populations of cardiac mitochondria. $A m \mathrm{~J}$ Physiol Heart Circ Physiol 289: H868-872, 2005.

116. Robinson $\mathbf{A B}$ and Rudd CJ. Deamidation of glutaminyl and asparaginyl residues in peptides and proteins. Curr Top Cell Regul 8: 247-295, 1974.

117. Rolo AP and Palmeira CM. DM and mitochondrial function: role of hyperglycemia and oxidative stress. Toxicology and applied pharmacology 212: 167178, 2006.

118. Rosca MG, Vazquez EJ, Kerner J, Parland W, Chandler MP, Stanley W, Sabbah HN, and Hoppel CL. Cardiac mitochondria in heart failure: decrease in respirasomes and oxidative phosphorylation. Cardiovasc Res 80: 30-39, 2008. 
119. Scheffler IE. Mitochondrial electron transport and oxidative phosphorylation. Mitochondria: 141-245, 1999.

120. Schlame M, Rua $\mathbf{D}$, and Greenberg $\mathbf{M L}$. The biosynthesis and functional role of cardiolipin. Prog Lipid Res 39: 257-288, 2000.

121. Schlame M, Towbin JA, Heerdt PM, Jehle R, DiMauro S, and Blanck TJ. Deficiency of tetralinoleoyl-cardiolipin in Barth syndrome. Ann Neurol 51: 634-637, 2002.

122. Schnedl WJ, Ferber S, Johnson JH, and Newgard CB. STZ transport and cytotoxicity. Specific enhancement in GLUT2-expressing cells. DM 43: 1326-1333, 1994.

123. Schneider M, Vogt Weisenhorn DM, Seiler A, Bornkamm GW, Brielmeier M, and Conrad M. Embryonic expression profile of phospholipid hydroperoxide glutathione peroxidase. Gene Expr Patterns 6: 489-494, 2006.

124. Schroeder JL, Luger-Hamer M, Pursley R, Pohida T, Chefd'hotel C, Kellman $\mathbf{P}$, and Balaban RS. Short communication: Subcellular motion compensation for minimally invasive microscopy, in vivo: evidence for oxygen gradients in resting muscle. Circ Res 106: 1129-1133, 2010.

125. Sedlak E and Robinson NC. Phospholipase A(2) digestion of cardiolipin bound to bovine cytochrome c oxidase alters both activity and quaternary structure. Biochemistry 38: 14966-14972, 1999.

126. Semeniuk LM, Kryski AJ, and Severson DL. Echocardiographic assessment of cardiac function in diabetic $\mathrm{db} / \mathrm{db}$ and transgenic $\mathrm{db} / \mathrm{db}$-hGLUT4 mice. Am J Physiol Heart Circ Physiol 283: H976-982, 2002.

127. Sentman ML, Granstrom M, Jakobson H, Reaume A, Basu S, and Marklund SL. Phenotypes of mice lacking extracellular superoxide dismutase and copper- and zinc-containing superoxide dismutase. J Biol Chem 281: 6904-6909, 2006.

128. Severson DL. Diabetic cardiomyopathy: recent evidence from mouse models of type 1 and type 2 DM. Canadian journal of physiology and pharmacology 82: 813-823, 2004.

129. Shen E, Li Y, Shan L, Zhu H, Feng Q, Arnold JM, and Peng T. Rac1 is required for cardiomyocyte apoptosis during hyperglycemia. DM 58: 2386-2395, 2009. 
130. Shen X, Zheng S, Metreveli NS, and Epstein PN. Protection of cardiac mitochondria by overexpression of MnSOD reduces diabetic cardiomyopathy. DM 55: 798-805, 2006.

131. Shen X, Zheng S, Thongboonkerd V, Xu M, Pierce WM, Jr., Klein JB, and Epstein PN. Cardiac mitochondrial damage and biogenesis in a chronic model of type 1 DM. Am J Physiol Endocrinol Metab 287: E896-905, 2004.

132. Shimada T, Horita K, Murakami M, and Ogura R. Morphological studies of different mitochondrial populations in monkey myocardial cells. Cell and tissue research 238: 577-582, 1984.

133. Shuvaeva TM, Novoselov VI, Fesenko EE, and Lipkin VM. [Peroxiredoxins, a new family of antioxidant proteins]. Bioorg Khim 35: 581-596, 2009.

134. Skulachev VP. Role of uncoupled and non-coupled oxidations in maintenance of safely low levels of oxygen and its one-electron reductants. Q Rev Biophys 29: 169-202, 1996.

135. Skulachev VP. Uncoupling: new approaches to an old problem of bioenergetics. Biochim Biophys Acta 1363: 100-124, 1998.

136. Stuckey DJ, Carr CA, Tyler DJ, Aasum E, and Clarke K. Novel MRI method to detect altered left ventricular ejection and filling patterns in rodent models of disease. Magn Reson Med 60: 582-587, 2008.

137. Takahashi $\mathbf{M}$ and Hood DA. Protein import into subsarcolemmal and intermyofibrillar skeletal muscle mitochondria. Differential import regulation in distinct subcellular regions. The Journal of biological chemistry 271: 27285-27291, 1996.

138. Tominaga M. [Diagnostic criteria for DM mellitus]. Rinsho Byori 47: 901-908, 1999.

139. Trost SU, Belke DD, Bluhm WF, Meyer M, Swanson E, and Dillmann WH. Overexpression of the sarcoplasmic reticulum $\mathrm{Ca}(2+)$-ATPase improves myocardial contractility in diabetic cardiomyopathy. DM 51: 1166-1171, 2002.

140. Turko IV and Murad F. Quantitative protein profiling in heart mitochondria from diabetic rats. J Biol Chem 278: 35844-35849, 2003.

141. U.S Department of Health and Human Resources CfDCaP. National DM Fact Sheet:general infomation and national estimates on DM in the united states, 2007. 
142. Ursini F, Heim S, Kiess M, Maiorino M, Roveri A, Wissing J, and Flohe L. Dual function of the selenoprotein PHGPx during sperm maturation. Science 285: 13931396, 1999.

143. Ursini F, Maiorino M, Brigelius-Flohe R, Aumann KD, Roveri A, Schomburg D, and Flohe L. Diversity of glutathione peroxidases. Methods Enzymol 252: 38-53, 1995.

144. Van den Bergh A, Vanderper A, Vangheluwe P, Desjardins F, Nevelsteen I, Verreth W, Wuytack F, Holvoet P, Flameng W, Balligand JL, and Herijgers P. Dyslipidaemia in type II diabetic mice does not aggravate contractile impairment but increases ventricular stiffness. Cardiovasc Res 77: 371-379, 2008.

145. Villanueva DS, Poirier P, Standley PR, and Broderick TL. Prevention of ischemic heart failure by exercise in spontaneously diabetic BB Wor rats subjected to insulin withdrawal. Metabolism 52: 791-797, 2003.

146. Vreken P, Valianpour F, Nijtmans LG, Grivell LA, Plecko B, Wanders RJ, and Barth PG. Defective remodeling of cardiolipin and phosphatidylglycerol in Barth syndrome. Biochem Biophys Res Commun 279: 378-382, 2000.

147. Way KJ, Isshiki K, Suzuma K, Yokota T, Zvagelsky D, Schoen FJ, Sandusky GE, Pechous PA, Vlahos CJ, Wakasaki H, and King GL. Expression of connective tissue growth factor is increased in injured myocardium associated with protein kinase $\mathrm{C}$ beta2 activation and DM. DM 51: 2709-2718, 2002.

148. Weiss JS and Sumpio BE. Review of prevalence and outcome of vascular disease in patients with DM mellitus. Eur J Vasc Endovasc Surg 31: 143-150, 2006.

149. Wende AR and Abel ED. Lipotoxicity in the heart. Biochim Biophys Acta 1801: 311-319, 2010.

150. Williamson CL, Dabkowski ER, Baseler WA, Croston TL, Alway SE, and Hollander JM. Enhanced apoptotic propensity in diabetic cardiac mitochondria: influence of subcellular spatial location. Am J Physiol Heart Circ Physiol 298: H633-642, 2010.

151. Wold LE, Ceylan-Isik AF, and Ren J. Oxidative stress and stress signaling: menace of diabetic cardiomyopathy. Acta pharmacologica Sinica 26: 908-917, 2005. 
152. Yamagishi SI, Edelstein D, Du XL, Kaneda Y, Guzman M, and Brownlee M. Leptin induces mitochondrial superoxide production and monocyte chemoattractant protein-1 expression in aortic endothelial cells by increasing fatty acid oxidation via protein kinase A. J Biol Chem 276: 25096-25100, 2001.

153. Yant LJ, Ran Q, Rao L, Van Remmen H, Shibatani T, Belter JG, Motta L, Richardson A, and Prolla TA. The selenoprotein GPX4 is essential for mouse development and protects from radiation and oxidative damage insults. Free Radic Biol Med 34: 496-502, 2003.

154. Ye G, Metreveli NS, Ren J, and Epstein PN. Metallothionein prevents DMinduced deficits in cardiomyocytes by inhibiting reactive oxygen species production. $D M$ 52: 777-783, 2003.

155. Yoshioka M, Kayo T, Ikeda T, and Koizumi A. A novel locus, Mody4, distal to D7Mit189 on chromosome 7 determines early-onset NIDDM in nonobese C57BL/6 (Akita) mutant mice. DM 46: 887-894, 1997.

156. Zarich SW and Nesto RW. Diabetic cardiomyopathy. American heart journal 118: 1000-1012, 1989.

157. Zhang M, Mileykovskaya E, and Dowhan W. Gluing the respiratory chain together. Cardiolipin is required for supercomplex formation in the inner mitochondrial membrane. J Biol Chem 277: 43553-43556, 2002. 


\section{Chapter 2:}

\section{Diabetic Cardiomyopathy-Associated Dysfunction in Spatially Distinct Mitochondrial Subpopulations}

As published in Am J Physiol Heart Circ Physiol. 2009 Feb; 296(2):H359-69.

Erinne R. Dabkowski ${ }^{1}$, Courtney L. Williamson ${ }^{1}$, Valerie C. Bukowski ${ }^{3}$, Rebecca S. Chapman $^{3}$, Stephen S. Leonard ${ }^{3}$, Cody J. Peer ${ }^{2}$, Patrick S. Callery ${ }^{2}$, and John M. Hollander ${ }^{1}$

\footnotetext{
${ }^{1}$ West Virginia University School of Medicine, Division of Exercise Physiology and Center for Interdisciplinary Research in Cardiovascular Sciences, Morgantown, WV 26506

${ }^{2}$ West Virginia University School of Pharmacy, Department of Basic Pharmaceutical Sciences, Morgantown, WV 26506

${ }^{3}$ National Institute for Occupational Safety and Health (NIOSH), Health Effects Laboratory Division, Morgantown, WV 26506
}

Running Title: Mitochondria and Diabetic Cardiomyopathy 


\section{ABSTRACT}

Cardiomyopathy is the leading cause of heart failure among diabetic patients, and mitochondrial dysfunction has been implicated as an underlying cause in the pathogenesis. Cardiac mitochondria consist of two spatially, functionally, and morphologically distinct subpopulations, termed subsarcolemmal mitochondria (SSM) and interfibrillar mitochondria (IFM). SSM are situated beneath the plasma membrane, whereas IFM are embedded between myofibrils. The goal of this study was to determine whether spatially distinct cardiac mitochondrial subpopulations respond differently to a diabetic phenotype. Swiss-Webster mice were subjected to intraperitoneal injections of streptozotocin or citrate saline vehicle. Five weeks after injections, diabetic hearts displayed decreased rates of contraction, relaxation, and left ventricular developed pressures ( $\mathrm{P}<0.05$ for all three). Both mitochondrial size (forward scatter, $\mathrm{P}<0.01$ ) and complexity (side scatter $<0.01$ ) were decreased in diabetic IFM but not diabetic SSM. Electron transport chain complex II respiration was decreased in diabetic SSM $(P<0.05)$ and diabetic IFM $(P<0.01)$, with the decrease being greater in IFM. Furthermore, IFM complex I respiration and complex III activity were decreased with diabetes $(P<0.01)$ but were unchanged in SSM. Superoxide production was increased only in diabetic IFM $(P<0.01)$. Oxidative damage to proteins and lipids, indexed through nitrotyrosine residues and lipid peroxidation, were higher in diabetic IFM ( $P<0.05$ and $P<0.01$, respectively). The mitochondria-specific phospholipid cardiolipin was decreased in diabetic IFM $(P<0.01)$ but not SSM. These results indicate that diabetes mellitus imposes a greater stress on the IFM subpopulation, which is associated, in part, with increased superoxide generation and oxidative damage, resulting in morphological and functional abnormalities that may contribute to the pathogenesis of diabetic cardiomyopathy 


\section{INTRODUCTION}

Diabetic cardiomyopathy has been implicated as a primary cause of heart failure among diabetic patients and it is thought to occur independent of vascular pathogenesis $(22,27,39,52,63)$. The hyperglycemic environment presented by diabetes mellitus leads to enhanced reactive oxygen species (ROS) generation and though many potential sources of ROS exist, the mitochondrion is considered to be the primary site $(6,8,15,28,50)$. Several sites in the electron transport chain (ETC) are particularly prone to the formation of ROS and include oxidizable electron carriers in the inner mitochondrial membrane $(12,32)$. This has implications for ETC proteins because a major constituent of these structures is their iron-sulphur centers (39), which can react with ROS such as superoxide $\left(\mathrm{O}_{2}{ }^{-}\right)$or hydrogen peroxide $\left(\mathrm{H}_{2} \mathrm{O}_{2}\right)$ and produce the highly reactive hydroxyl radical $(\cdot \mathrm{OH})(14)$. Increased mitochondrial ROS production has been linked to mitochondrial dysfunction $(13,50,51,53,54)$ which can in turn alter the integrity of the inner mitochondrial membrane, facilitating further dysfunction in the mitochondria. In particular, proteins and lipids within the mitochondria are critical targets of elevated ROS production, and their oxidative modification potentiates mitochondrial dysfunction, by limiting adequate production of ATP. One potential target of mitochondrial dysfunction is the mitochondrial-specific phospholipid, cardiolipin. Cardiolipin is a diphosphatidylglycerol enriched in the inner membrane, containing oxidatively-sensitive acyl groups that may act as selective targets for $\operatorname{ROS}(33,34,46)$. Cardiolipin interacts with a number of mitochondrial proteins including $\mathrm{F}_{0} \mathrm{~F}_{1}$-ATPase, ANT, cytochrome $C$, and ETC complexes I, III, and IV, and its oxidative modification may be a critical event for apoptosis initiation (23).

The cardiac myocyte contains two distinct mitochondrial subpopulations that are characterized by their spatial arrangement within the cell. These two disparate populations have been termed subsarcolemmal mitochondria (SSM) and interfibrillar mitochondria (IFM) due to their subcellular locations which either abut the sarcolemma or situate between the contractile apparatus $(44,49)$. In addition to spatial differences, 
mitochondrial subpopulations differ in structure, size, ATP levels, protein import rates, substrate utilization, and other biochemical properties $(1,21,26,28,31,34,38,44)$. Morphologically, IFM are smaller, more compact and possess functionally greater respiratory rates, supplying ATP mainly for contractile function. SSM are larger, more variable in shape, producing ATP primarily for electrolyte and protein transport across the plasma membrane (44). The two mitochondrial subpopulations respond differently to physiological stimuli, including exercise, aging, obesity, fasting, apoptotic initiators, and ischemia/reperfusion (I/R) injury $(1,31,34,38,48,57)$. Decreased ETC function and elevated oxidative stress has been reported in SSM following myocardial I/R, with no difference observed in the IFM (33). Using electron microscopy, Kelley et al. observed decreased IFM size in skeletal muscle of type 2 diabetic patients, which was not observed in SSM (28). Others have observed decreased IFM ETC function with aging $(21,26)$. These findings indicate that though mitochondria are similar in their central role in cellular function, spatially distinct populations are influenced by pathological states differently, requiring careful examination of individual mitochondrial subpopulations.

Because much of the previous work examining the impact of diabetes mellitus on mitochondrial disposition has been performed on total mitochondria, it is difficult to assess the impact of the pathology on spatially distinct mitochondrial populations. The goal of this study was to determine the differential response of individual mitochondrial subpopulations subjected to a diabetic phenotype, to further our understanding of their specific contribution to the pathogenesis of diabetic cardiomyopathy. Our results indicate that the IFM subpopulation is affected by diabetes mellitus to a greater extent than SSM as reflected by greater morphological changes, elevated ROS, and enhanced oxidative damage. This study is the first to determine the spatial influence of the diabetic phenotype on cardiac mitochondrial dysfunction. 


\section{MATERIALS AND METHODS}

\section{Experimental Animals and Diabetes Induction}

The animal experiments in this study conformed to the National Institutes of Health $(\mathrm{NIH})$ guidelines for the care and use of laboratory animals and were approved by the West Virginia University Animal Care and Use Committee (WVU-ACUC). Female Swiss Webster mice (Harlan, Indianapolis, IN), were housed in the West Virginia University Health Sciences Center animal facility. Mice were given unlimited access to a rodent diet and water. Diabetes was induced in eight week old mice following the protocol of the Animal Models of Diabetic Complications Consortium (AMDCC), using multiple low-dose streptozotocin (STZ; Sigma, St. Louis, MO) injections. A multiple low-dose STZ protocol was chosen, because previous reports indicate that this model limits the body weight losses associated with diabetic protocols $(24,29)$. Injections of $50 \mathrm{mg} / \mathrm{kg}$ body weight STZ dissolved in sodium citrate buffer $(\mathrm{pH}$ 4.5), were performed daily for five consecutive days following six hours of fasting. Mice that served as vehicle controls were given the same volume per body weight of sodium citrate buffer. One week post injection, hyperglycemia was confirmed by measuring urinary glucose (Chemstrip 2GP Urine test strips, Roche Diagnostics, Indianapolis, IN), where $>2000 \mathrm{mg} / \mathrm{dL}$ was considered diabetic. To confirm the diabetic phenotype urinary glucose levels were monitored weekly using a DiaScreen 50 Urine Chemistry Analyzer (Arkray, Edina, MN), and DiaScreen 10 reagent strips. Five weeks post hyperglycemia onset, animals were sacrificed for further experimentation.

\section{Cardiac Contractile Function}

Hearts from diabetic and control animals were isolated and transferred to a Langendorff set-up for contractile studies as previously described $(19,20,59)$. In brief, hearts were removed from anesthetized mice, and immersed in cold cardioplegic solution. After cannulation of the aorta on a luer stub, hearts were perfused retrograde at $37^{\circ} \mathrm{C}$ with a modified Krebs-Henseleit buffer (in $\mathrm{mmol} / \mathrm{L}$ : 0.5 pyruvate, 0.4 caprylic 
acid, $118 \mathrm{NaCl}, 4.7 \mathrm{KCl}, 2.25 \mathrm{CaCl}_{2}, 1.2 \mathrm{MgSO}_{4}, 1.2 \mathrm{KH}_{2} \mathrm{PO}_{4}, 25 \mathrm{NaHCO}_{3}, 0.5$ $\mathrm{Na}_{2}$ EDTA, 5.5 glucose) that was subject to oxygenation. A small fluid-filled balloon was inserted into the left ventricular (LV) cavity and coupled to a pressure transducer (Argon Medical Devices, Athens, TX). The balloon was inflated until the end-diastolic pressure reached $10 \mathrm{mmHg}$. Platinum wires were placed on the surface of the right atrium and used to pace the heart at 420 beats $/ \mathrm{min}(7 \mathrm{~Hz})$ with increasing voltage until the heart rate was consistent (captured). During this time period, hearts remained submerged in a $37^{\circ} \mathrm{C}$ jacketed chamber, and a heat lamp attached to a variable $A C$ transformer was utilized to maintain temperature which was monitored by a needle temperature probe inserted just prior to the luer cannula (Physitemp, Clifton, NJ). Hearts were perfused for 15 minutes to achieve a stable cardiac function, and then followed for an additional 45 minutes yielding a total protocol time of 1 hour. During this time, digitized recordings of the LV pressure and its first derivative, as well as heart rate were captured on an IBMcompatible PC using ChartPro software (AD Instruments, Colorado Springs, CO). LV peak systolic pressure (PSP), developed pressure (DP), maximum speed of contraction $(+\mathrm{dP} / \mathrm{dt})$, and maximum speed of relaxation $(-\mathrm{dP} / \mathrm{dt})$ were analyzed from the recordings using ChartPro software.

\section{Preparation of Individual Mitochondrial Subpopulations}

Five weeks following hyperglycemia onset, animals were sacrificed and hearts removed. Hearts were rinsed in phosphate buffered saline (PBS, pH 7.4), then blotted dry and weighed. Subsarcolemmal mitochondria (SSM) and interfibrillar mitochondria (IFM) were isolated on ice following the methods of Palmer et al. with minor modifications (44). Briefly, the ventricles were minced and homogenized 1:10 (w/v) in cold Chappel-Perry buffer (in mmol/L: $100 \mathrm{KCl}, 40$ Tris $\mathrm{HCl}, 10$ Tris-Base, $5 \mathrm{MgCl}_{2}, 1$ ATP, 1 EDTA, pH 7.4). The homogenates were than centrifuged at $800 \times g$ for 10 minutes. The supernatant was extracted and centrifuged again at $9,000 \times g$ to isolate the SSM. The SSM pellet was washed and centrifuged two more times at 9,000 $\times g$ and once more at $5,000 \times g$ to obtain a clean SSM fraction. The remaining pellet from the 
$800 \times g$ spin was resuspended in Buffer 2 (in mmol/L: $100 \mathrm{KCl}, 5 \mathrm{MgSO}_{4}, 5 \mathrm{EGTA}, 50$ Tris $\mathrm{HCl}, \mathrm{pH} \mathrm{7.4)}$ and exposed to $5 \mathrm{mg} / \mathrm{kg}$ of trypsin for 10 minutes (49). After 10 minutes, the IFM pellet was diluted two-fold with buffer and spun down at 5,000 $\mathrm{g} g$ for 5 minutes. The supernatant was discarded and the pellet was resuspended in buffer and spun down at $800 \times g$ for 10 minutes. The supernatant was saved and the pellet resuspended and spun down again at $800 \times g$ for 10 minutes to maximize IFM yield. Next, supernatants were combined and spun down at 9,000 $\times g$ to yield the IFM. IFM were washed several times and spun down at a final spin of 5,000 $\mathrm{x} g$ for 10 minutes. Pellets were resuspended in a sucrose buffer (in mmol/L: 220 sucrose, 70 mannitol, 10 Tris $\mathrm{HCl}, 1$ EDTA, $\mathrm{pH} 7.4$ ) and protein concentrations were determined using the Bradford method and bovine serum albumin as a standard (4). Superoxide $\left(\mathrm{O}_{2}{ }^{-}\right)$ production, oxygen consumption, nitrotyrosine protein and lipid peroxidation were assessed on freshly isolated subfractions.

\section{Mitochondria Size and Internal Complexity}

To index mitochondrial subpopulation size and complexity, we performed flow cytometry analyses using a FACS Calibur equipped with a $15 \mathrm{mw} 488 \mathrm{~nm}$ argon laser and 633 red diode laser (Becton and Dickinson, San Jose, CA, USA) as previously described (8, 9). Each individual parameter (gating, size, and complexity) was performed using specific light sources (laser, photomultiplier tube) and specific detectors. MitoTracker Deep Red 633 (Invitrogen, Carlsbad, CA) which moves into intact mitochondria due to membrane potential, was used to selectively stain intact mitochondria (EM: $633 \mathrm{~nm}$; fluorescent 633 red diode laser) and exclude debris which contains no membrane potential, enabling accurate gating (R1) of the mitochondria. Once the gating parameters were established, gated events $(20,000$ per sample) were subsequently examined using the forward scatter detector (FSC; $488 \mathrm{~nm}$ argon laser and diode detector) and side scatter detector (SSC; photomultiplier tube and $90^{\circ}$ collection lens) and represented in FSC vs. SSC density plots. Geometric mean (arbitrary units, AU) representing FSC (logarithmic scale) was used as an indicator of 
size while values from SSC (logarithmic scale) were used to indicate complexity in the subpopulations. Though the FSC arbitrary unit is proportional to the individual mitochondria particle size, the absolute value still remains an arbitrary unit. Thus, to confirm the absolute mitochondria size, we utilized a flow cytometry size calibration kit (Invitrogen, Carlsbad, CA) which utilizes a set of microsphere suspensions $(0.5 \mu \mathrm{m}-6$ $\mu \mathrm{m})$ to serve as reliable size references for flow cytometric analyses. All flow cytometric measurements were performed under the supervision of the West Virginia University Flow Cytometry Core Facility.

\section{Mitochondrial Cardiolipin Content}

Cardiolipin was determined as previously described (16). Briefly, isolated mitochondrial subpopulations $(200 \mu \mathrm{g})$ were incubated with a synthetic phospholipid 1,1',2,2'- tetramyristoylcardiolipin (T14:0 CL; Avanti Polar Lipids, Alabaster, AL) that served as an internal standard. Total lipids, including the internal standard, were extracted using the method of Bligh and Dyer (3). Briefly, $\mathrm{MeOH} / \mathrm{H}_{2} \mathrm{O}$ and chloroform were added to the sample, mixed, and then centrifuged at approximately $200 \times g$ for 10 minutes. The chloroform layer was extracted from each sample then dried under a nitrogen stream. Each sample was resuspended in $4 \mathrm{~mL}$ of chloroform:MeOH (1:1) and washed with $1.8 \mathrm{~mL}$ of $20 \mathrm{mM} \mathrm{LiCl}$ aqueous solution. The organic layer was dried under a nitrogen stream and the residue resuspended in $1 \mathrm{~mL}$ of chloroform and then filtered through a $0.2 \mu \mathrm{m}$ PTFE syringe filter into a $5 \mathrm{~mL}$ glass centrifuge tube. The filtrate was filtered a second time with a $0.2 \mu \mathrm{m}$ PTFE syringe filter and then dried under a nitrogen stream. Samples were resuspended in $500 \mu \mathrm{L} / \mathrm{mg}$ protein in chloroform/MeOH (1:1) and diluted further before injection into the mass spectrometer. $\mathrm{LiOH}$ (50 nmol/mg of protein) was added to each sample just prior to analysis.

Cardiolipin detection was performed by electrospray ionization mass spectrometry (ESI-MS) using a quadrupole ion trap Finnigan LCQ DECA (ThermoFisher, Waltham, MA) operated in the negative-ion mode. Mass spectrometer instrument conditions included a spray voltage of $5.2 \mathrm{kV}$, capillary voltage of $-4 \mathrm{~V}$, 
heated capillary temperature of $300{ }^{\circ} \mathrm{C}$, and a sheath gas $\left(\mathrm{N}_{2}\right)$ flow rate of 40 arbitrary units. The cardiolipin solution was infused using a $100 \mu \mathrm{L}$ syringe at a flow rate of $5 \mu \mathrm{L}$ per minute. Mitochondrial cardiolipin and internal standard spectra were identified in the full scan mode. The peak intensities of cardiolipin:internal standard were estimated using single ion monitoring on doubly charged m/z 723 and m/z 619 in both diabetic and control mitochondria.

\section{Electron Transport Chain Respiration}

State 4 respiratory capacity was assessed in isolated mitochondrial subpopulations following the method of Hofhaus et al. (18), with slight modifications (19). Following mitochondrial subpopulation isolation, samples were resuspended in respiration buffer (in mmol/L: 20 Hepes, $10 \mathrm{MgCl}_{2}, 250$ sucrose) and equal volumes were loaded into a Gilson chamber (Gilson, Middleton, WI) attached to a YSI 5300 biological oxygen monitor (YSI Inc. Yellow Springs, $\mathrm{OH}$ ). Respiration through complex I, complex III, and complex IV were determined by measuring the rate of oxygen consumed in the presence of specific substrates. These substrates included glutamate/ malate for complex I, succinate for complex II and, ascorbate/N, N, N', N'-tetramethyl-pphenylenediamine (TMPD) for complex IV and was calculated as the fraction that was sensitive to the specific inhibitors rotenone (complex I), antimycin (complex III), and sodium cyanide (complex IV). Assessment of complex III respiration was performed spectrophotometrically as previously described by following the reduction of cytochrome $C$ in the presence of reduced decylubiquinone (60). Protein content was determined following the Bradford method as above, and values expressed as nanomoles of $\mathrm{O}_{2}$ consumed per minute, per mg protein (complex 1, II, IV), or nanomoles substrate per minute per mg protein (complex III). 


\section{Electron Paramagnetic Resonance (EPR) Spectroscopy}

An EPR spin trapping technique was used to detect short-lived free radicals such as hydroxyl $(\cdot \mathrm{OH})$ and superoxide $\left(\mathrm{O}_{2}^{-}-\right)(62)$. This method is based on the reaction of a short-lived radical binding with a paramagnetic compound to form a relatively long-lived free radical product (spin adduct). This adduct can then be observed using conventional EPR whereby the intensity of the signal is used to measure the amount of short-lived radicals trapped. The hyperfine couplings of the spin adduct are generally characteristics of the original trapped radicals. This method is ideal for detection and identification of free radicals because of its specificity and sensitivity. All EPR measurements were conducted using a Bruker EMX spectrometer (Bruker Instruments Inc., Billerica, MA) and a flat cell assembly. Hyperfine couplings were measured (to 0.1 G) directly from magnetic field separation using potassium tetraperoxochromate $\left(\mathrm{K}_{3} \mathrm{CrO}_{8}\right)$ and 1,1-diphenyl-2-picrylhydrazyl (DPPH) as reference standards $(5,25)$. The Acquisit program was used for data acquisitions and analyses (Bruker Instruments Inc., Billerica, MA 01821). Isolated heart mitochondria subpopulations were resuspended in $500 \mu$ of $1 \times$ PBS ( $\mathrm{pH} 7.4$ ). One hundred $\mu \mathrm{g}$ of each sample was brought up to $200 \mu \mathrm{L}$ with PBS and incubated with the spin trap 5,5-dimethyl-1-pyrroline-N-oxide (DMPO, 200 $\mathrm{mM}$ ) in the presence or absence of excess complex I respiratory substrates glutamate and malate reaching an end volume of $500 \mu \mathrm{L}$. Samples were incubated for three minutes at $37^{\circ} \mathrm{C}$ and then transferred to an EPR flat cell for measurement at room temperature with instrument settings of 63.6 milliwatt, modulation amplitude 1G, receiver gain $1.00 \times 10^{5}$, conversion time $40.960 \mathrm{~ms}$, and time constant $40.960 \mathrm{~ms}$. All spectra shown are in accumulation of three scans. The reaction of xanthine and xanthine oxidase was used as a reference. The relative radical concentration was estimated by measuring the peak-to-peak height $(\mathrm{mm})$ of the observed spectra.

\section{Protein Nitrotyrosine Content}

Oxidatively- modified proteins were examined by measuring nitrosylated groups introduced into protein side chains using a commercially available kit (Cell Sciences, 
Canton, MA). Nitrotyrosine-containing proteins were measured using a solid-phase enzyme-linked immunosorbent assay based on the sandwich principle. Samples were incubated in microtiter wells coated with antibodies recognizing nitrotyrosine residues. Following incubation and washing, a biotinylated secondary antibody (tracer) was added, followed by washing and addition of a streptavidin-peroxidase conjugate. Color development was measured spectrophotometrically at $450 \mathrm{~nm}$, following addition of tetramethylbenzidine (TMB) using a Biotek Synergy HT plate reader (Biotek, Winooski, $\mathrm{VT}$ ), and values were compared against known nitrotyrosine standards.

\section{Lipid Peroxidation Products}

Peroxidation of lipids was assessed by measurement of malondialdehyde (MDA) and 4-hydroxyalkenals (4-HAE), stable end products formed from the oxidation of polyunsaturated fatty acids and esters. Equal volumes of freshly isolated mitochondrial subpopulations were analyzed for MDA and 4-HAE by using a colorimetric assay kit (Oxford Biomedical Research Company, Oxford, MI). This assay is based on the reaction of a chromogenic reagent, N-methyl-2-phenylindole with MDA and 4-HAE at $45^{\circ} \mathrm{C}$. One molecule of either MDA or 4-HAE reacts with two molecules of the reagent to yield a stable chromophore with maximal absorbance at $586 \mathrm{~nm}$. Absorbance was measured on a Biotek Synergy HT plate reader (Biotek, Winooski, VT), and protein content was assessed as above with final values expressed per $\mathrm{mg}$ of protein.

\section{Statistics}

Means and standard errors were calculated for all data sets. Data were analyzed with a one-way analysis of variance (ANOVA) method to evaluate the main treatment effect, diabetes induction (Systat; version 5.03, Evanston, IL). Fisher's Least Significant Difference (LSD) post hoc tests were performed to determine the significant differences among means. When appropriate a Student's T-test was employed. $P<0.05$ was considered significant. 


\section{RESULTS}

\section{Cardiac Contractile Function in the Diabetic Heart}

Heart weight, body weight, and heart weight/body weight ratios were not significantly altered following STZ treatment (Table 2.1) which is in agreement with other studies utilizing multiple low-dose STZ protocols $(24,29)$. Analyses of non-fasting urinary ketone levels five weeks after the onset of diabetes were negative for diabetic animals (data not shown). Five weeks after the onset of diabetes and hyperglycemia, animals were sacrificed and the heart removed for measurement of contractile function using a Langendorff perfusion apparatus. Rates of contraction $(+\mathrm{dP} / \mathrm{dt})$ and relaxation $(-\mathrm{dP} / \mathrm{dt})$, as well as developed pressures (DP) were significantly decreased in diabetic vs. control hearts (Table 2.1; $P<0.05$, for all three), demonstrating decreased contractile function. These data are in agreement with others examining the influence of the diabetic phenotype using STZ injection as a model $(15,52,56,58)$.

\section{Mitochondrial Subpopulation Morphology}

Mitochondrial subpopulations were isolated, and yields were similar between control and diabetic hearts (Table 2.1). To determine morphological differences between control and diabetic mitochondrial subpopulations, we used a novel flow cytometry approach in which gating was performed to make an accurate estimation of mitochondrial size and complexity. Assessment of forward-scattered light (FSC) was used to estimate size, while assessment of side-scattered light (SSC), was used to estimate mitochondrial complexity, both of which were based on a logarithmic scale. Individual mitochondria subpopulations were stained with MitoTracker Deep Red 633, which incorporates into intact mitochondria. In Figure 2.1A, a typical dot plot, showing MitoTracker Deep Red 633 stained mitochondria is shown, with intact mitochondria indicated in red and unstained debris indicated in black. Using this information, we gated the mitochondria (R1) to exclude unstained debris, and applied the R1 gate to analyses on the individual subpopulations. These analyses lend insight into relative 
morphological differences between the two subpopulations, and help to confirm success of the isolation procedure. To confirm differences in absolute mitochondria size, we included size calibration beads composed of microsphere suspensions ranging in size from $0.5 \mu \mathrm{m}$ to $6 \mu \mathrm{m}$ to serve as reliable size references. Using this approach, SSM were larger in size (FSC) and possessed greater internal complexity (SSC) as compared to IFM, which were smaller and more compact (Figures 2.1B and 2.1C). These results are consistent with previously published reports $(28,48,49,55)$. Mitochondrial size was significantly decreased by $35 \%(P<0.01)$ in the diabetic IFM as compared to control IFM, while SSM showed no significant changes (Figure 2.1D). Mitochondrial complexity in the diabetic IFM was also significantly decreased, by $40 \%$ $(P<0.01)$, as compared to control IFM (Figure 2.1E). No significant differences in SSC were observed in the SSM population (Figure 2.1E). These results indicate that only IFM morphology is impacted as a result of STZ-induced diabetic insult.

\section{Electron Transport Chain (ETC) Respiration}

Mitochondrial ETC respiration is altered with hyperglycemia but it is unclear whether these alterations are uniform between mitochondrial subpopulations. We used polarography to assess changes in ETC respiration within individual mitochondrial subpopulations from diabetic and control hearts. Complex I, II, and III were significantly decreased in diabetic IFM, relative to control (Figure 2.2A and 2.2B, $P<0.01$, for both; Figure $2.2 \mathrm{C} ; P<0.05$ ), while only complex II was significantly decreased in the SSM (Figure 2.2B; $P<0.05$ ). No significant changes were observed with complex IV in either

subpopulation (Figure 2.2D). These data indicate that STZ-induced diabetic insult impacts both SSM and IFM, but these effects are greater in IFM. 


\section{Reactive Oxygen Species (ROS) Production}

Because the mitochondrion is centrally involved in the formation of ROS, we determined whether the diabetic phenotype enhanced ROS formation in a subpopulation specific manner. Mitochondrial subpopulations were incubated with complex I substrates glutamate/malate to fuel the ETC, and ROS generation analyzed using EPR spectroscopy. We observed increases in diabetic IFM spin-trapping peaks, relative to control IFM (Figure 2.3C, 2.3D, and 2.3H; $P<0.01$ ), and these differences were not observed in SSM (Figures 2.3A, 2.3B, and 2.3H). The spin-trapping pattern observed was indicative of $\mathrm{O}_{2}^{-}-$, and addition of superoxide dismutase (SOD) confirmed the observed spectra as $\mathrm{O}_{2}{ }^{-}$- (Figures $2.3 \mathrm{E}$ and 2.3F). A spectrum for xanthine oxidase, a known $\mathrm{O}_{2}{ }^{-}$- generator, is included for spin-trapping pattern comparison (Figure 2.3G). These data suggest that ROS generation is significantly increased in the IFM, which may be the result of enhanced $\mathrm{O}_{2}{ }^{-}$- production.

\section{Oxidative Damage to Proteins and Lipids}

To determine whether oxidative stress levels are greater in a specific mitochondrial subpopulation, we examined protein nitrotyrosine contents, as well as levels of lipid peroxidation breakdown products malondialdehyde (MDA) and 4hydroxyalkenal (4-HAE), in both mitochondrial subpopulations following diabetic insult. Nitrotyrosine content was significantly greater in the diabetic IFM relative to control, and no significant difference was observed with the SSM (Figure 2.4, $P<0.05$ ). MDA and 4HAE were significantly higher in both diabetic subpopulations as compared to control (Figure 2.5, $P<0.01$ for SSM; $P<0.005$ for IFM). These data indicate that STZ-induced diabetic insult enhances protein modification, as a result of nitrosylation in diabetic IFM, with no significant impact on SSM. Further, lipid peroxidation is enhanced in both mitochondrial subpopulations, but this increase may be greater in diabetic IFM. 


\section{Mitochondrial Cardiolipin Content}

Cardiolipin is a phospholipid unique to the mitochondrial inner membrane that is a diphosphatidylglycerol, containing oxidatively-sensitive acyl groups that may act as selective targets for ROS. Depending on pathological state, cardiolipin content within mitochondrial subpopulations has been shown to be affected differently (34). Because cardiolipin oxidation is susceptible to ROS (46), we examined whether diabetic cardiomyopathy is associated with mitochondrial subpopulation-specific alteration, and thus subcellular spatial influence. Mass spectrometric analyses were performed to detect alterations in concentrations of the most abundant cardiolipin molecular species, (T18:2 cardiolipin; $m / z 723$ ) (15). Cardiolipin and its internal standard gave both singlyand doubly-charged molecules (Figures 6A-D). Cardiolipin was detected based on its doubly-charged signal at $m / z 723$ and its singly-charged ion at $m / z 1447$. The internal standard (T14:0 CL) was detected based upon doubly- and singly-charged signals at $\mathrm{m} / \mathrm{z} 619$ and $\mathrm{m} / \mathrm{z}$ 1238, respectively. A ratio of cardiolipin:internal standard was calculated by single ion monitoring of both $\mathrm{m} / \mathrm{z} 723$ and $\mathrm{m} / \mathrm{z} 619$ to determine the change in cardiolipin levels between control and diabetic samples. Using this approach, we observed a significant decrease of approximately $60 \%$ in diabetic IFM as compared to controls (Figures $6 \mathrm{C}, 6 \mathrm{D}$, and $6 \mathrm{E} ; P<0.01$ ). In contrast, no significant differences were observed in diabetic SSM, as compared to controls (Figure 6A, 6B, and 6E). These data indicate that in response to the diabetic phenotype, cardiolipin content is significantly decreased in the IFM subpopulation, with no significant change in the SSM. 


\section{DISCUSSION}

Diabetes mellitus is associated with a cardiomyopathy that is independent of atherosclerosis and characterized by abnormal ventricular contractile function $(2,15$, $22,27,52,63)$. Diabetic cardiomyopathy has been shown to progress to heart failure in both type 1 and type 2 diabetic models, though at a much higher rate in the type 1 setting $(15,52)$. Using a well described and utilized model of diabetes induction, STZ injection, we sought to determine the effect of diabetic insult on cardiac contractile function. STZ treatment had no significant impact on heart weight, body weight, or heart weight:body weight ratios (Table 2.1). In contrast, STZ treatment significantly decreased rates of contraction $(+d P / d t)$, rates of relaxation $(-d P / d t)$, and developed pressure (DP), as compared to citrate saline-injected controls (Table 2.1). Our observations are in agreement with others who have observed similar cardiac contractile deficits in the diabetic context, characteristic of diabetic cardiomyopathy $(2$, $15,22,52,63)$.

As the primary source of energy for the cardiac myocyte, mitochondria play a central role in cellular homeostasis. Not surprisingly, disruption of this critical organelle is regarded as a key contributor to the development of pathological states, including diabetic cardiomyopathy $(50,53,54)$. Nevertheless, examination of mitochondria is complicated by the fact that two mitochondrial subpopulations are present in the cardiac myocyte, interfibrillar mitochondria (IFM), which situate between the contractile apparatus and subsarcolemmal mitochondria (SSM), which exist beneath the plasma membrane. These two disparate mitochondrial subpopulations are distinguished by specific spatial arrangements, distinct functional properties, and differential responses to pathological conditions. In general, SSM have been reported to be larger and more complex internally, while the IFM tend to be smaller, elongated and densely packed between the myofibrils $(21,31,38,44)$. Using flow cytometric analyses, we observed morphological differences between SSM and IFM that were similar to other reports utilizing electron microscopy in cells and in isolated mitochondria (Figure 2.1) $(44,49)$. Examination of mitochondrial subpopulations from hearts subjected to STZ-treatment revealed decreases in both size and complexity of the IFM population, with no 
significant effect on the SSM population (Figure 2.1). Our findings are in agreement with those of Ritov et al. who observed decreases in the size of mitochondria located along the $Z$ line (presumably reflective of IFM) in the vastus lateralis of type 2 diabetic patients (28). In contrast, Shen et al. observed mitochondrial swelling in a novel model of type 1 diabetes (the OVE26 mouse), suggestive of an enhanced apoptotic program (54). These conflicting results may be due to a number of differences between the studies. The OVE26 mouse model utilizes transgenic modification to alter calmodulin levels in the pancreatic $\beta$ cell, precipitating a profound early-onset diabetic phenotype (10). Further, OVE26 transgenic mice are examined at approximately 4 months of age, a substantially longer time period of diabetes exposure than our current study of 5 weeks. It is possible that mitochondria display a biphasic morphological response during diabetic insult, characterized by an initial decrease in size, followed by an increase in size. In addition, examination of the total mitochondria fraction may be incapable of resolving the differences between individual mitochondrial subpopulations. In either case, these data indicate that mitochondria display changes in overall morphology as a result of diabetic insult, and that these changes may be specific for a distinct subpopulation.

Because of its enhanced propensity for ROS-mediated damage, mitochondrial ETC function can be profoundly affected by oxidative stress-associated pathologies, including diabetes mellitus. We have previously observed changes in ETC respiration during diabetic insult (8), which is in agreement with other reports $(28,30)$. Specifically, we observed significant decreases in oxygen consumption at both complex I and complex III, but not at complex IV (8). However, examination of the effect of diabetes on individual mitochondrial subpopulations revealed a differential response with decreased respiratory activity in complex I, II, and III in IFM, while only complex II respiration was decreased in SSM (Figure 2.2). Though not significant, we observed a decrease in complex IV respiratory activity of diabetic IFM (Figure 2.2 C). These results are similar to others that have found decreased complex III and complex IV respiration in the IFM population with no effect in the SSM population, in response to aging (11, 22). Taken together, our findings indicate that ETC complex II may be a specific locus for the deleterious effects associated with STZ insult, imparting damage to both IFM and 
SSM subpopulations, while effects on complex I and III may be specific to the IFM. Our results suggest that with type 1 diabetes mellitus, ETC respiratory activities are compromised impacting both subpopulations with the effects being greatest in the IFM. Because the IFM population provides ATP for the contractile process, the compromise in ETC function of this mitochondrial population may be particularly detrimental to cardiac contractile function, contributing to the contractile deficits associated with diabetic cardiomyopathy.

It has been suggested that an increase in ROS in response to hyperglycemia is the proximal defect that leads to many of the subsequent pathological consequences resulting from diabetes mellitus $(6,13,28,50)$. Studies indicate that various ROS are increased during diabetes mellitus in the heart, including $\mathrm{O}_{2}^{-}-(7,35)$, peroxynitrite (OONO'-) (43), $\cdot \mathrm{OH}(41,45)$, and hydrogen peroxide $\left(\mathrm{H}_{2} \mathrm{O}_{2}\right)(36)$. Using the nonspecific fluorometric probe, dichlorofluorescein (DCF), we previously observed an increase in ROS production in total diabetic mitochondria (8). Our results are in agreement with others observing similar increases in ROS presence resulting from diabetic insult, using DCF as a probe (50). However, because the DCF probe detects ROS in a non-specific manner, we extended our findings by determining the specific reactants that are increased in cardiac diabetic mitochondria, using EPR/spin trapping methodology. Our results indicate that diabetic insult significantly increase $\mathrm{O}_{2}{ }^{\circ}$ - only in the IFM, suggesting that increased ROS occurs in a subpopulation specific manner (Figure 2.3). Verification of $\mathrm{O}_{2}{ }^{-}$- presence was accomplished by addition of exogenous superoxide dismutase (SOD) which essentially abolished the signal (Figure 3E and 3F). Comparison of the observed spectra to the spectra of a known $\mathrm{O}_{2}{ }^{*}$ - generator, xanthine oxidase (Figure 2.3G), provided further confirmation to the nature of the reactant. These results are interesting indicating that enhanced ROS presence as a result of diabetes mellitus, is particularly relevant in the IFM subpopulation and that $\mathrm{O}_{2}{ }^{-}$- appears to be a primary ROS generated in diabetic mitochondria. Our findings are similar to others examining different cardiac pathologies using DCF detection or amplex red assay $(26,57)$. Moghaddas et al. found an increase in $\mathrm{H}_{2} \mathrm{O}_{2}$ from complex III in the IFM with aging, and no differences in the SSM (37). Indirectly, our results indicating enhanced $\mathrm{O}_{2}:$ - presence are also in agreement with Shen et al. who observed 
restoration of mitochondrial function in MnSOD transgenic mice crossed with OVE26 mice (53). It should be pointed out that the primary sites for ROS generation in the mitochondrion are at complex $\mathrm{I}$ and III, thus decreases in respiration of these complexes, as observed in the IFM, should limit electron flux into complex III attenuating ROS generation. Our results do not support this scenario, but rather may be the function of enhanced oxidative damage to proteins in these complexes such that, though electron flux through these complexes is decreased, electron leakage is greater resulting in amplification of ROS generation. The results warrant further investigation.

Overproduction of ROS represents an initial event in the pathogenesis of diabetic cardiomyopathy creating an oxidative imbalance in the cell. The resulting oxidative milieu promotes damage to subcellular components such as membranes and proteins. Submitochondrial targets are particularly prone to damage from enhanced ROS due to their close proximity to the source of generation. Because we observed increases in mitochondrial ROS generation as a result of STZ exposure, we examined oxidative modification to both protein and lipid components in individual mitochondrial subpopulations to determine whether oxidative damage occurs in subpopulation specific manner. Assessment of protein modification was performed by analysis of nitrotyrosine adducts. Our results indicate that only proteins from the IFM population display enhanced nitrosylated proteins in response to STZ exposure (Figure 2.4). These findings are similar with others who have demonstrated enhanced protein carbonyl groups in cardiac IFM, as a result of aging (26). Interestingly, these authors also observed an increase in protein carbonyls in the SSM population as a result of aging, though this increase was not as great as that observed in the IFM (26). The reason for these differences may be related to the pathologies studied (aging vs. diabetes mellitus), and/or the specific protein modification examined (nitrotyrosine vs. carbonyl). Lipid peroxidation was determined by the combined assessment of both MDA and 4HAE (Figure 2.5). Our results indicate that STZ exposure increases lipid peroxidation in both the SSM and IFM subpopulations, though the increase may be greater in the IFM (Figure 2.5). Our findings are somewhat in agreement with others who have observed increases in lipid peroxidation primarily in cardiac IFM as a result of aging $(26,57)$. Of particular interest in the current study was our observation that STZ exposure enhanced 
lipid peroxidation in both subpopulations, though the increase appeared to be larger in the IFM. Our findings are in contrast to previous reports and may be a function of the different pathologies examined and/or assay methodology $(26,57)$. These studies examined lipid peroxidation in aging cardiac mitochondrial subpopulations by assessing 4-HAE via Western blot analyses and/or MDA via spectrophotometric measurement of thiobarbituric acid reactive substances (TBARS). In our current study, we assessed lipid peroxidation by assessing a combination of MDA and 4-HAE spectrophotometrically, using the chromogenic reagent, $\mathrm{N}$-methyl-2-phenylindole. Additionally, oxidative stress to mitochondrial lipid components may be different in the aging context as compared to diabetic insult. It is important to point out that while STZ effects are believed to be primarily associated with the pancreas its short-term administration has been associated with ROS generation and oxidative damage to other tissues such as kidney and liver (47). Thus, one must consider the potential for a direct effect of STZ on the heart, independent of enhanced glucose content resulting from pancreatic $\beta$-cell loss.

Diabetic mitochondria display increases in mitochondrial permeability transition pore (mPTP) opening (42), as well as changes in mitochondrial membrane fluidity, which may contribute to changes in mitochondrial membrane potential $\left(\Delta \psi_{m}\right)$ (61). These effects may be influenced by phospholipid content and in particular, cardiolipin, which would have a profound impact on mitochondrial function (16). Cardiolipin is a diphosphatidylglycerol enriched in the inner membrane, containing oxidatively-sensitive acyl groups that may act as selective targets for ROS $(17,40)$. Cardiolipin interacts with a number of mitochondrial proteins including components of the MPTP, cytochrome $C$, and ETC complexes, thus, its oxidative modification may be a critical event for the initiation of pathological states (23). We determined whether cardiolipin was a specific lipid target of STZ-induced diabetic insult. Our findings indicate that cardiolipin loss occurs as a result of STZ exposure only in the IFM subpopulation (Figure 6E). Our results are in contrast to those of Lesnefsky et al. who observed a selective decrease in cardiolipin content in SSM following I/R insult (34). These authors suggest that SSM possess a decreased capacity, relative to IFM, for calcium $\left(\mathrm{Ca}^{2+}\right)$ accumulation which is a hallmark of I/R insult. As a result, SSM sustain a faster onset for ischemic damage as 
compared to IFM (34). Though increased $\mathrm{Ca}^{2+}$ content may be involved in the pathogenesis of diabetes mellitus, its involvement has much greater effects during myocardial I/R, which may account for the difference in the results observed in the studies.

In conclusion, we report for the first time, that STZ-induced diabetic insult differentially affects morphological, functional, and oxidative properties of spatially distinct mitochondrial subpopulations. Type 1 diabetic insult is associated with a greater stress on the IFM subpopulation as indicated by increased ROS and oxidative damage, impacting ETC function and cardiolipin content, all of which may contribute to cardiac contractile dysfunction. The heterogeneous response displayed by individual mitochondrial subpopulations during diabetic insult emphasizes the importance of incorporating spatial influence into the study of mitochondria in disease states. 


\section{ACKNOWLEDGEMENTS}

This work was supported by National Institutes of Health Award \# DP2DK083095 (J. M. H.) from the National Institutes of Diabetes and Digestive and Kidney Diseases

(NIDDK). This work was also supported by American Heart Association Beginning Grant-In-Aid \#0665237B (J. M. H.) and Grant-In-Aid \#0855484D (J. M. H.). Erinne Dabkowski is a recipient of an American Heart Association Predoctoral Fellowship (\#0815406D). We would like to thank Dr. Christopher Cuff and the contributions from the West Virginia University Flow Cytometry Core facility. Flow cytometry studies were supported in part by grants \#RR020866 and \#RR16440.

Disclaimer: The findings and conclusions in this report are those of the author(s) and do not necessarily represent views of the National Institute for Occupational Safety and Health. 


\section{REFERENCES}

1. Adhihetty PJ, Ljubicic V, Menzies KJ, Hood DA. Differential susceptibility of subsarcolemmal and intermyofibrillar mitochondria to apoptotic stimuli. Am J Physiol Cell Physiol 289: C994-C1001, 2005.

2. An D, Rodrigues $\mathbf{B}$. Role of changes in cardiac metabolism in development of diabetic cardiomyopathy. Am J Physiol Heart Circ Physiol 291: H1489-H1506, 2006.

3. Bligh EG, Dyer WJ. A rapid method of total lipid extraction and purification. Can J Biochem Physiol 37: 911-917, 1959.

4. Bradford MM. A rapid and sensitive method for the quantitation of microgram quantities of protein utilizing the principle of protein-dye binding. Anal Biochem 72: 248-254, 1976.

5. Buettner GR. Spin trapping: ESR parameters of spin adducts. Free Radic Biol Med 3: 259-303, 1987.

6. Cai L, Li W, Wang G, Guo L, Jiang Y, Kang YJ. Hyperglycemia-induced apoptosis in mouse myocardium: mitochondrial cytochrome C-mediated caspase-3 activation pathway. Diabetes 51: 1938-1948, 2002.

7. Cai L, Wang J, Li Y, Sun X, Wang L, Zhou Z, Kang YJ. Inhibition of superoxide generation and associated nitrosative damage is involved in metallothionein prevention of diabetic cardiomyopathy. Diabetes 54: 1829-1837, 2005.

8. Dabkowski ER, Williamson CL, Hollander JM. Increased susceptibility of mitochondrial subpopulations to diabetic cardiomyopathy associated dysfunction. FASEB J 21: 732, 2007. 
9. Dabkowski ER, Williamson CL, Hollander JM. Mitochondria-specific transgenic overexpression of phospholipid hydroperoxide glutathione peroxidase (GPx4) attenuates ischemia/reperfusion-associated cardiac dysfunction. Free Radic Biol Med 45: 855-865, 2008.

10. Epstein PN, Overbeek PA, Means AR. Calmodulin-induced early-onset diabetes in transgenic mice. Cell 58: 1067-1073, 1989.

11. Fannin SW, Lesnefsky EJ, Slabe TJ, Hassan MO, Hoppel CL. Aging selectively decreases oxidative capacity in rat heart interfibrillar mitochondria. Arch Biochem Biophys 372: 399-407, 1999.

12. Ferrari R, Ceconi C, Curello S, Cargnoni A, De Giuli F, Visioli O. Occurrence of oxidative stress during myocardial reperfusion. Mol Cell Biochem 111: 61-69, 1992.

13. Flarsheim CE, Grupp IL, Matlib MA. Mitochondrial dysfunction accompanies diastolic dysfunction in diabetic rat heart. Am J Physiol Heart Circ Physiol 271: H192-H202, 1996.

14. Fridovich I. Superoxide radical and superoxide dismutases. Annu Rev Biochem 64: 97-112, 1995.

15. Hamblin M, Friedman DB, Hill S, Caprioli RM, Smith HM, Hill MF. Alterations in the diabetic myocardial proteome coupled with increased myocardial oxidative stress underlies diabetic cardiomyopathy. J Mol Cell Cardiol 42: 884-895, 2007.

16. Han X, Yang J, Cheng H, Yang K, Abendschein DR, Gross RW. Shotgun lipidomics identifies cardiolipin depletion in diabetic myocardium linking altered substrate utilization with mitochondrial dysfunction. Biochemistry 44: 1668416694, 2005.

17. Hoch FL. Cardiolipins and biomembrane function. Biochim Biophys Acta 1113: 71-133, 1992. 
18. Hofhaus G, Shakeley RM, Attardi G. Use of polarography to detect respiration defects in cell cultures. Methods Enzymol 264: 476-483, 1996.

19. Hollander JM, Lin KM, Scott BT, Dillmann WH. Overexpression of PHGPx and HSP60/10 protects against ischemia/reoxygenation injury. Free Radic Biol Med 35: 742-751, 2003.

20. Hollander JM, Martin JL, Belke DD, Scott BT, Swanson E, Krishnamoorthy V, Dillmann WH. Overexpression of wild-type heat shock protein 27 and a nonphosphorylatable heat shock protein 27 mutant protects against ischemia/reperfusion injury in a transgenic mouse model. Circulation 110: 35443552, 2004.

21. Hoppel CL, Moghaddas S, Lesnefsky EJ. Interfibrillar cardiac mitochondrial complex III defects in the aging rat heart. Biogerontology 3: 41-44, 2002.

22. Hoppel CL, Tandler B, Parland W, Turkaly JS, Albers Hamster cardiomyopathy LD. A defect in oxidative phosphorylation in the cardiac interfibrillar mitochondria. J Biol Chem 257: 1540-1548, 1982.

23. Imai H, Nakagawa Y. Biological significance of phospholipid hydroperoxide glutathione peroxidase (PHGPx, GPx4) in mammalian cells. Free Radic Biol Med 34: 145-169, 2003.

24. Inada A, Kanamori H, Arai H, Akashi T, Araki M, Weir GC, Fukatsu A. A model for diabetic nephropathy: advantages of the inducible cAMP early repressor transgenic mouse over the streptozotocin-induced diabetic mouse. $J$ Cell Physiol 215: 383-391, 2008.

25. Janzen E, Blackburn B. Detection and identification of short-lived free radicals by and electron spin resonance trapping technique. J Am Chem Soc 90: 59095910, 1968. 
26. Judge S, Jang YM, Smith A, Hagen T, Leeuwenburgh C. Age-associated increases in oxidative stress and antioxidant enzyme activities in cardiac interfibrillar mitochondria: implications for the mitochondrial theory of aging. FASEB J 19: 419-421, 2005.

27. Kannel WB, McGee DL. Diabetes and cardiovascular disease. The Framingham study. JAMA 241: 2035-2038, 1979.

28. Kelley DE, He J, Menshikova EV, Ritov VB. Dysfunction of mitochondria in human skeletal muscle in type 2 diabetes. Diabetes 51: 2944-2950, 2002.

29. Kim E, Sohn S, Lee M, Jung J, Kineman RD, Park S. Differential responses of the growth hormone axis in two rat models of streptozotocin-induced insulinopenic diabetes. J Endocrinol 188: 263-270, 2006.

30. King KL, Young ME, Kerner J, Huang H, O'Shea KM, Alexson SE, Hoppel CL, Stanley WC. Diabetes or peroxisome proliferator-activated receptor alpha agonist increases mitochondrial thioesterase I activity in heart. J Lipid Res 48: 1511-1517, 2007.

31. Koves TR, Noland RC, Bates AL, Henes ST, Muoio DM, Cortright RN. Subsarcolemmal and intermyofibrillar mitochondria play distinct roles in regulating skeletal muscle fatty acid metabolism. Am J Physiol Cell Physiol 288: C1074-C1082, 2005.

32. Lashin O, Romani A. Mitochondria respiration and susceptibility to ischemiareperfusion injury in diabetic hearts. Arch Biochem Biophys 420: 298-304, 2003.

\section{Lesnefsky EJ, Chen Q, Slabe TJ, Stoll MS, Minkler PE, Hassan MO, Tandler} B, Hoppel CL. Ischemia, rather than reperfusion, inhibits respiration through cytochrome oxidase in the isolated, perfused rabbit heart: role of cardiolipin. $\mathrm{Am}$ J Physiol Heart Circ Physiol 287: H258-H267, 2004. 
34. Lesnefsky EJ, Slabe TJ, Stoll MS, Minkler PE, Hoppel CL. Myocardial ischemia selectively depletes cardiolipin in rabbit heart subsarcolemmal mitochondria. Am J Physiol Heart Circ Physiol 280: H2770-H2778, 2001.

35. Midaoui AE, Elimadi A, Wu L, Haddad PS, de Champlain J. Lipoic acid prevents hypertension, hyperglycemia, and the increase in heart mitochondrial superoxide production. Am J Hypertens 16: 173-179, 2003.

36. Mikrut K, Paluszak J, Kozlik J, Sosnowski P, Krauss H, Grzeskowiak E. The effect of bradykinin on the oxidative state of rats with acute hyperglycaemia. Diabetes Res Clin Pract 51: 79-85, 2001.

37. Moghaddas S, Stoll MS, Minkler PE, Salomon RG, Hoppel CL, Lesnefsky EJ. Preservation of cardiolipin content during aging in rat heart interfibrillar mitochondria. J Gerontol A Biol Sci Med Sci 57: B22-B28, 2002.

38. Mollica MP, Lionetti L, Crescenzo R, D'Andrea E, Ferraro M, Liverini G, lossa S. Heterogeneous bioenergetic behavior of subsarcolemmal and intermyofibrillar mitochondria in fed and fasted rats. Cell Mol Life Sci 63: 358366, 2006.

39. Newsholme E, ARL. Biochemistry for the Medical Sciences. Chichester: Wiley, 1983.

40. O'Brien PJ, Little C. Intracellular mechanisms for the decomposition of a lipid peroxide. II. Decomposition of a lipid peroxide by subcellular fractions. Can J Biochem 47: 493-499, 1969.

41. Ohkuwa T, Sato Y, Naoi M. Hydroxyl radical formation in diabetic rats induced by streptozotocin. Life Sci 56: 1789-1798, 1995.

42. Oliveira PJ, Seica R, Coxito PM, Rolo AP, Palmeira CM, Santos MS, Moreno AJ. Enhanced permeability transition explains the reduced calcium uptake in 
cardiac mitochondria from streptozotocin-induced diabetic rats. FEBS Lett 554: 511-514, 2003.

43. Pacher $\mathbf{P}$, Szabo $\mathbf{C}$. Role of peroxynitrite in the pathogenesis of cardiovascular complications of diabetes. Curr Opin Pharmacol 6: 136-141, 2006.

44.Palmer JW, Tandler B, Hoppel CL. Biochemical properties of subsarcolemmal and interfibrillar mitochondria isolated from rat cardiac muscle. J Biol Chem 252: 8731-8739, 1977.

45. Pennathur S, Wagner JD, Leeuwenburgh C, Litwak KN, Heinecke JW. A hydroxyl radical-like species oxidizes cynomolgus monkey artery wall proteins in early diabetic vascular disease. J Clin Invest 107: 853-860, 2001.

46. Petrosillo G, Ruggiero FM, Paradies G. Role of reactive oxygen species and cardiolipin in the release of cytochrome $c$ from mitochondria. FASEB J 17: 22022208, 2003.

47. Petzold GL, Swenberg JA. Detection of DNA damage induced in vivo following exposure of rats to carcinogens. Cancer Res 38: 1589-1594, 1978.

48. Ritov VB, Menshikova EV, He J, Ferrell RE, Goodpaster BH, Kelley DE. Deficiency of subsarcolemmal mitochondria in obesity and type 2 diabetes. Diabetes 54: 8-14, 2005.

49. Riva A, Tandler B, Loffredo F, Vazquez E, Hoppel C. Structural differences in two biochemically defined populations of cardiac mitochondria. Am J Physiol Heart Circ Physiol 289: H868-H872, 2005.

50. Rolo AP, Palmeira CM. Diabetes and mitochondrial function: role of hyperglycemia and oxidative stress. Toxicol Appl Pharmacol 212: 167-178, 2006. 
51. Santos DL, Palmeira CM, Seica R, Dias J, Mesquita J, Moreno AJ, Santos MS. Diabetes and mitochondrial oxidative stress: a study using heart mitochondria from the diabetic Goto-Kakizaki rat. Mol Cell Biochem 246: 163170, 2003.

52. Severson DL. Diabetic cardiomyopathy: recent evidence from mouse models of type 1 and type 2 diabetes. Can J Physiol Pharmacol 82: 813-823, 2000.

53. Shen X, Zheng S, Metreveli NS, Epstein PN. Protection of cardiac mitochondria by overexpression of MnSOD reduces diabetic cardiomyopathy. Diabetes 55 : 798-805, 2006.

54. Shen X, Zheng S, Thongboonkerd V, Xu M, Pierce WM Jr, Klein JB, Epstein PN. Cardiac mitochondrial damage and biogenesis in a chronic model of type 1 diabetes. Am J Physiol Endocrinol Metab 287: E896-E905, 2004.

55. Shimada T, Horita K, Murakami M, Ogura R. Morphological studies of different mitochondrial populations in monkey myocardial cells. Cell Tissue Res 238: 577582, 1984.

56. Stanley WC, Lopaschuk GD, McCormack JG. Regulation of energy substrate metabolism in the diabetic heart. Cardiovasc Res 34: 25-33, 1997.

57. Suh JH, Heath SH, Hagen TM. Two subpopulations of mitochondria in the aging rat heart display heterogeneous levels of oxidative stress. Free Radic Biol Med 35: 1064-1072, 2003.

58. Taegtmeyer H, McNulty $\mathbf{P}$, Young ME. Adaptation and maladaptation of the heart in diabetes: part I: general concepts. Circulation 105: 1727-1733, 2002.

59. Trost SU, Belke DD, Bluhm WF, Meyer M, Swanson E, Dillmann WH. Overexpression of the sarcoplasmic reticulum $\mathrm{Ca}^{2+}$-ATPase improves myocardial contractility in diabetic cardiomyopathy. Diabetes 51: 1166-1171, 2002. 
60. Trounce IA, Kim YL, Jun AS, Wallace DC. Assessment of mitochondrial oxidative phosphorylation in patient muscle biopsies, lymphoblasts, and transmitochondrial cell lines. Methods Enzymol 264: 484-509, 1996.

61. Waczulikova I, Habodaszova D, Cagalinec M, Ferko M, Ulicna O, Mateasik A, Sikurova L, Ziegelhoffer A. Mitochondrial membrane fluidity, potential, and calcium transients in the myocardium from acute diabetic rats. Can J Physiol Pharmacol 85: 372-381, 2007.

62. Wang Y, Fang J, Leonard SS, Rao KM. Cadmium inhibits the electron transfer chain and induces reactive oxygen species. Free Radic Biol Med 36: 1434-1443, 2004.

63. Zarich SW, Nesto RW. Diabetic cardiomyopathy. Am Heart J 118: 1000-1012, 1989. 
Figure 2.1
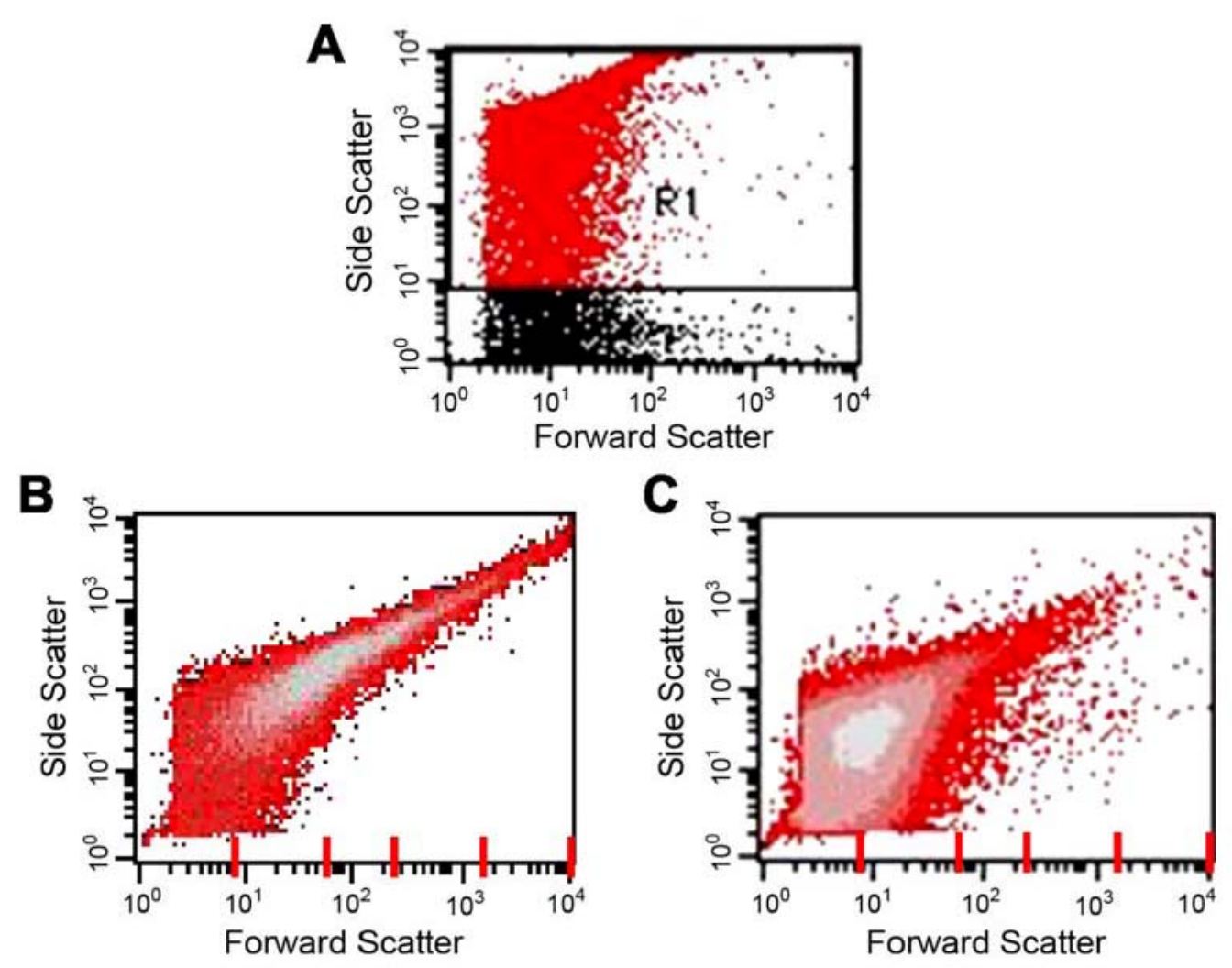
Figure 2.1
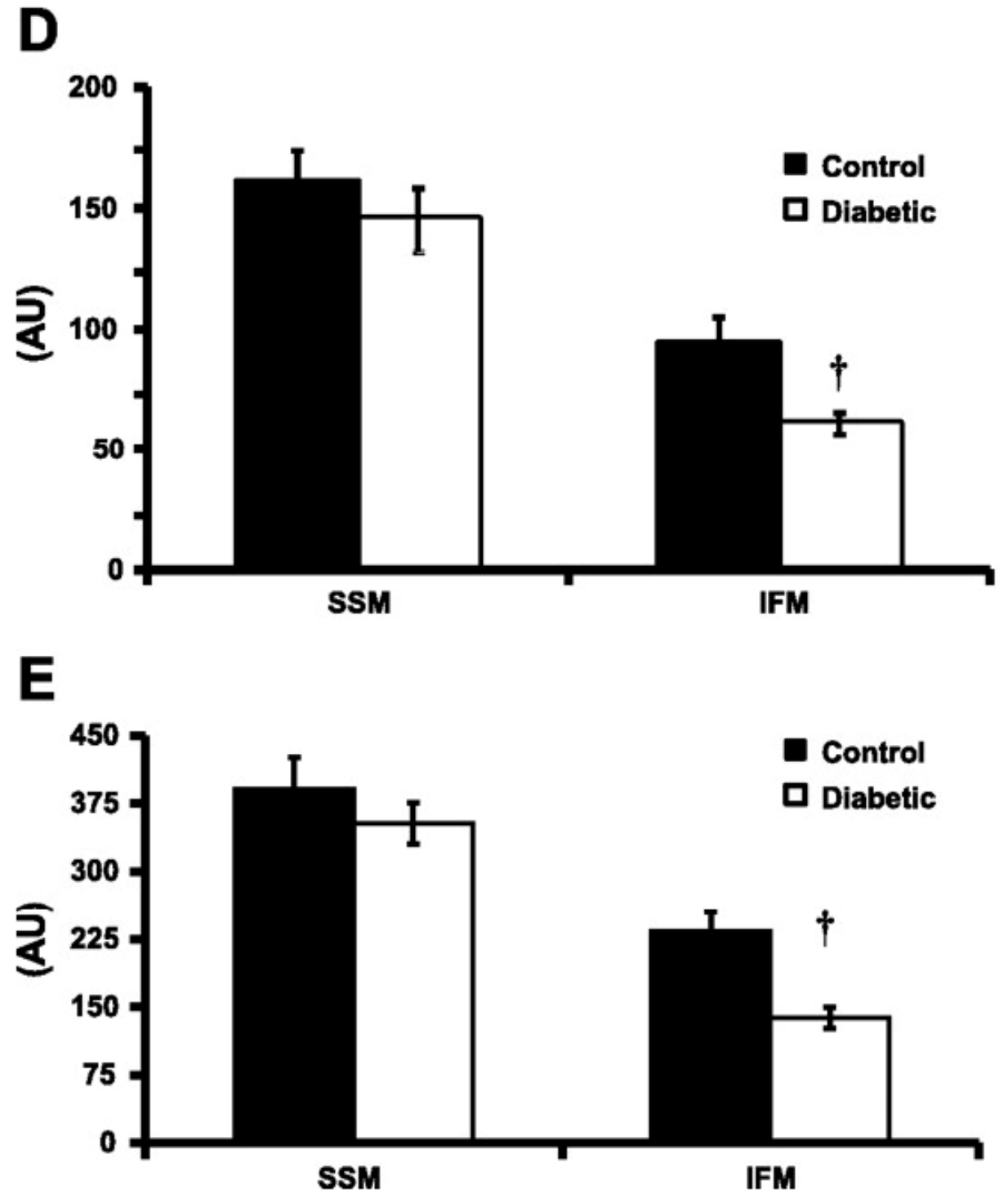
Figure 2.1. Mitochondria subpopulation morphological assessment. Determination of relative size and internal complexity of distinct mitochondrial subpopulations using flow cytometric analyses. Representative density plot of isolated mitochondria stained with MitoTracker Deep Red 633, which incorporates into intact mitochondria. Intact mitochondria that take up the MitoTracker Deep Red 633 dye are gated (R1) and represented in red. Noise and debris, which does not take up the MitoTracker Deep Red 633 is excluded from measurements (outside the R1 gate) and indicated in black. (B) Representative gated density plot indicating size (FSC; forward scatter) vs. internal complexity (SSC; side scatter) of isolated SSM. (C) Representative gated density plot indicating size (FSC; forward scatter) vs. internal complexity (SSC; side scatter) of isolated IFM. Analysis of forward scatter and side scatter were calculated per 20,000 gated events for all mitochondrial subpopulations. Red hash lines indicate microsphere size references from left to right; $0.5,1,2,4$, and $6 \mu \mathrm{M}$, respectively. (D) Analysis of cardiac SSM and IFM size (FSC) in control and diabetic. (E) Analysis of cardiac SSM and IFM complexity (SSM) in control and diabetic. Values for size and complexity are expressed as arbitrary units (AU) $\pm \mathrm{SEM} ; \mathrm{n}=3$ for each group. $\dagger P<0.01$ for diabetic vs. control. 
Figure 2.2

A

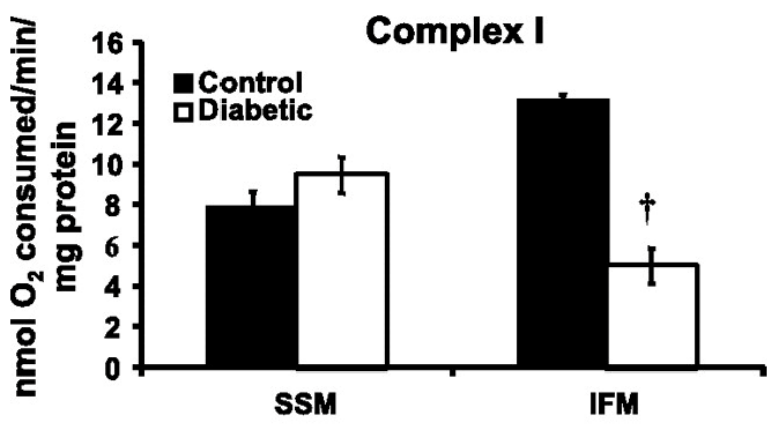

C

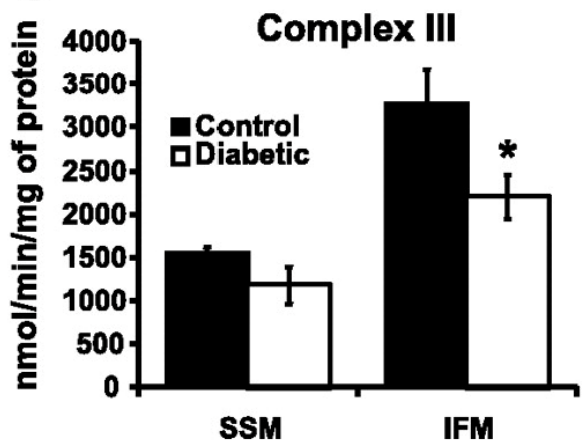

B

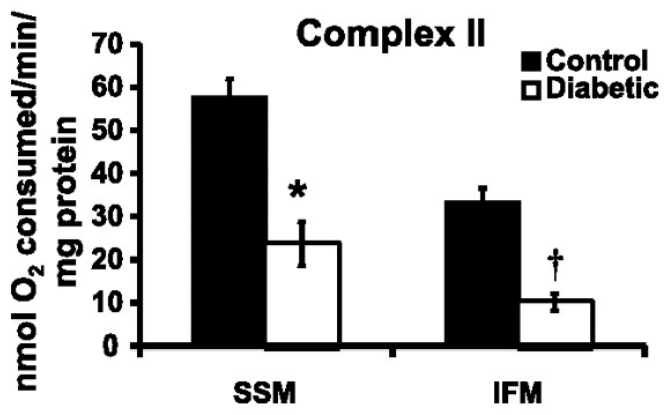

D

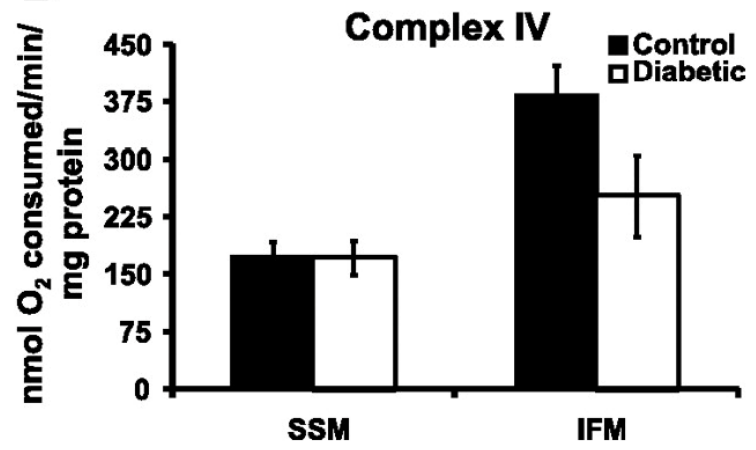


Figure 2.2. Mitochondria subpopulation respiration. Individual mitochondrial subpopulations were isolated from diabetic and control hearts and polarographic measurements performed to index oxygen consumption under state 4 respiration conditions. Respiration of individual ETC complexes was defined as the rate of oxygen consumed in the presence of specific substrates, glutamate/malate (complex I), succinate (complex II), and ascorbate/N,N,N',N'-tetramethyl-p-phenylenediamine (complex IV), and were calculated as the fraction that was sensitive to the specific inhibitors rotenone (complex I), antimycin (complex II), and sodium cyanide (complex IV). Measurement of complex III was performed spectrophotometrically by assessment of the reduction of cytochrome $c$ in the presence of reduced decylubiquinone. (A) Complex I. (B) Complex II. (C) Complex III. (D) Complex IV. Values are expressed as mean \pm SEM; $\mathrm{n}=7$ for each group. ${ }^{*} P<0.05$ for diabetic vs. control; $\dagger P<0.01$ for diabetic vs. control. 
Figure 2.3
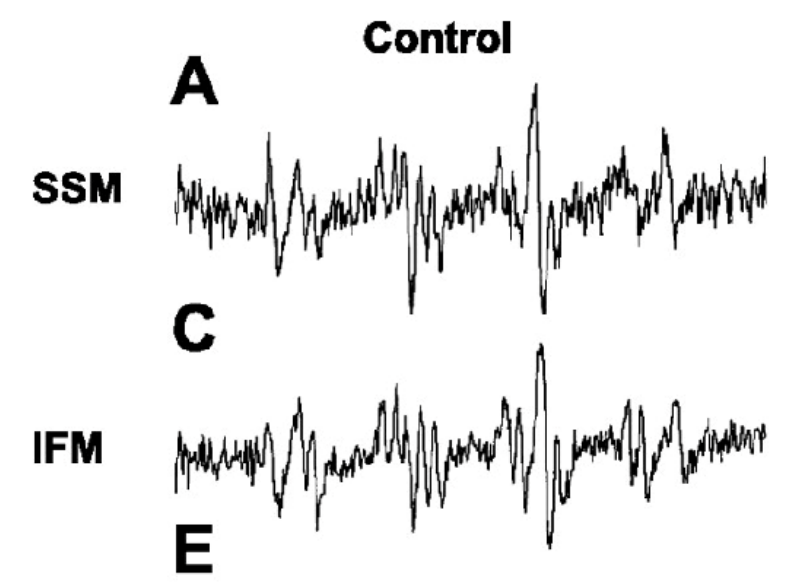

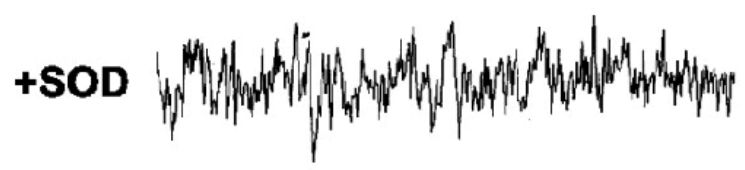
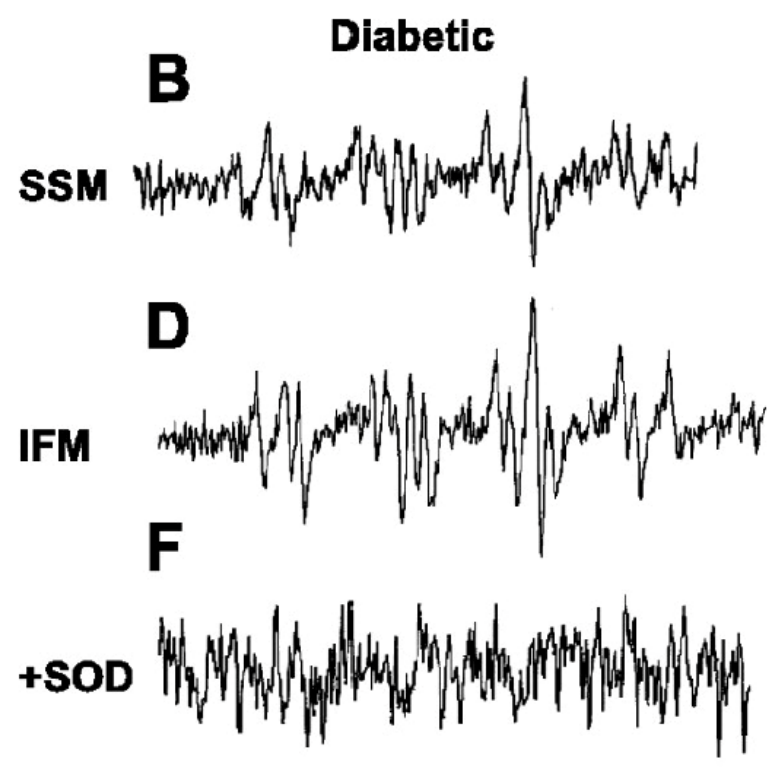

G

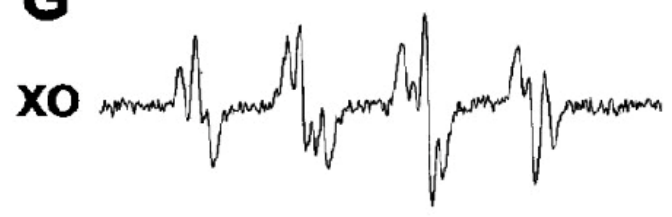


Figure 2.3

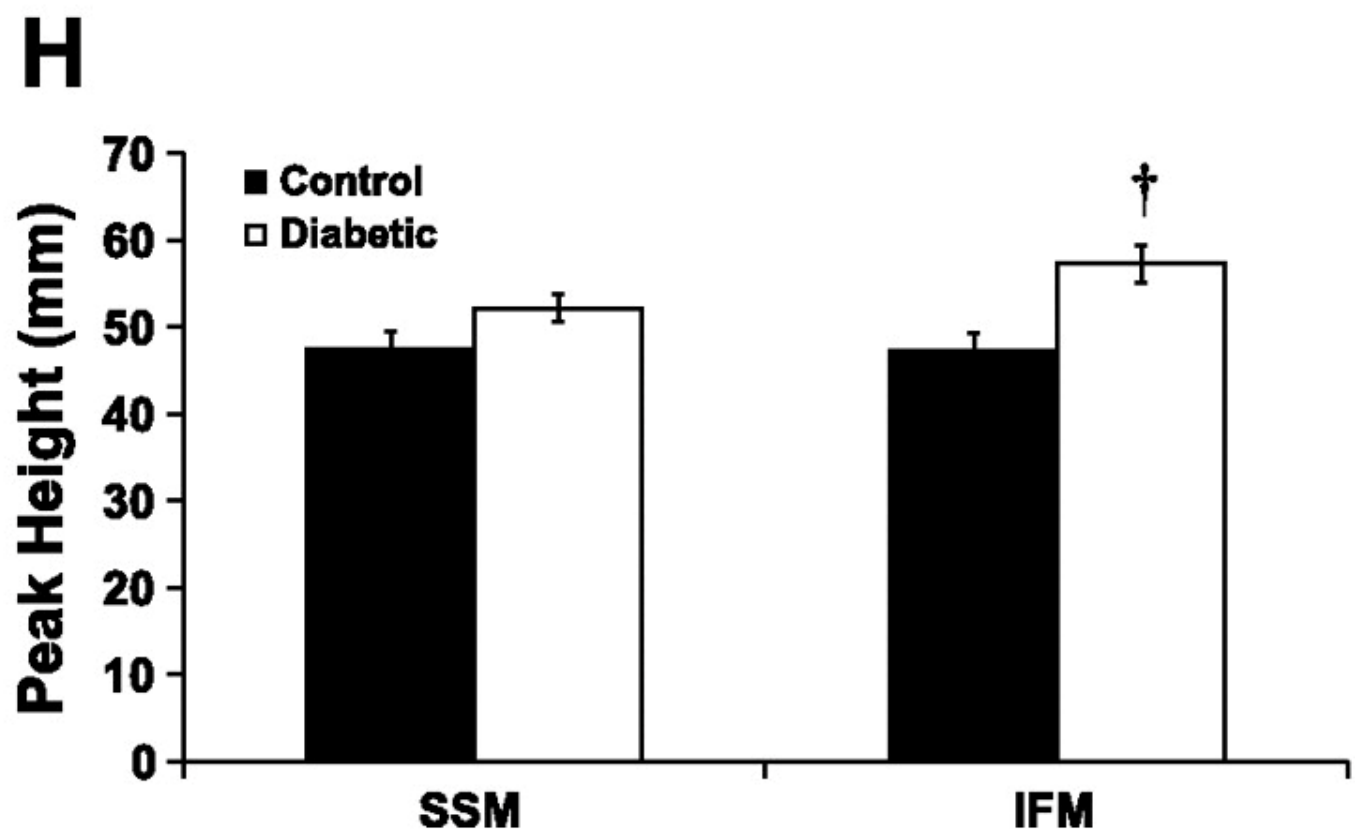


Figure 2.3. Mitochondria subpopulation reactive oxygen species (ROS) generation. Individual mitochondrial subpopulations were isolated from diabetic and control hearts and electron paramagnetic resonance (EPR) spectroscopy performed to index ROS generation. Isolated mitochondrial subpopulations were incubated with DMPO in the presence or absence of excess complex I respiratory substrates glutamate and malate, incubated for three minutes at $37^{\circ} \mathrm{C}$ and then transferred to an EPR flat cell for measurement at room temperature with instrument settings of 63.6 milliwatt, modulation amplitude $1 \mathrm{G}$, receiver gain $1.00 \times 10^{5}$, conversion time $40.960 \mathrm{~ms}$, and time constant $40.960 \mathrm{~ms}$. All spectra shown are in accumulation of three scans. (A) Representative spectra for control SSM; (B) representative spectra for diabetic SSM; (C) representative spectra for control IFM; (D) representative spectra for diabetic IFM; (E) Representative spectra for SSM with the inclusion of superoxide dismutase (SOD) into the assay medium to confirm the superoxide $\left(\mathrm{O}_{2}^{-}\right)$signal; (F) representative spectra for IFM with the inclusion of superoxide dismutase (SOD) into the assay medium to confirm the superoxide $\left(\mathrm{O}_{2}^{-}\right)$signal; $(\mathbf{G})$ xanthine oxidase spectra was included as a representative of the $\mathrm{O}_{2}$ - spectra pattern. $(\mathrm{H})$ ROS formation was significantly increased in diabetic IFM compared to control IFM. Values are expressed as mean $\pm \mathrm{SEM} ; \mathrm{n}=4$ for each group. $\dagger P<0.01$ for diabetic vs. control. 
Figure 2.4

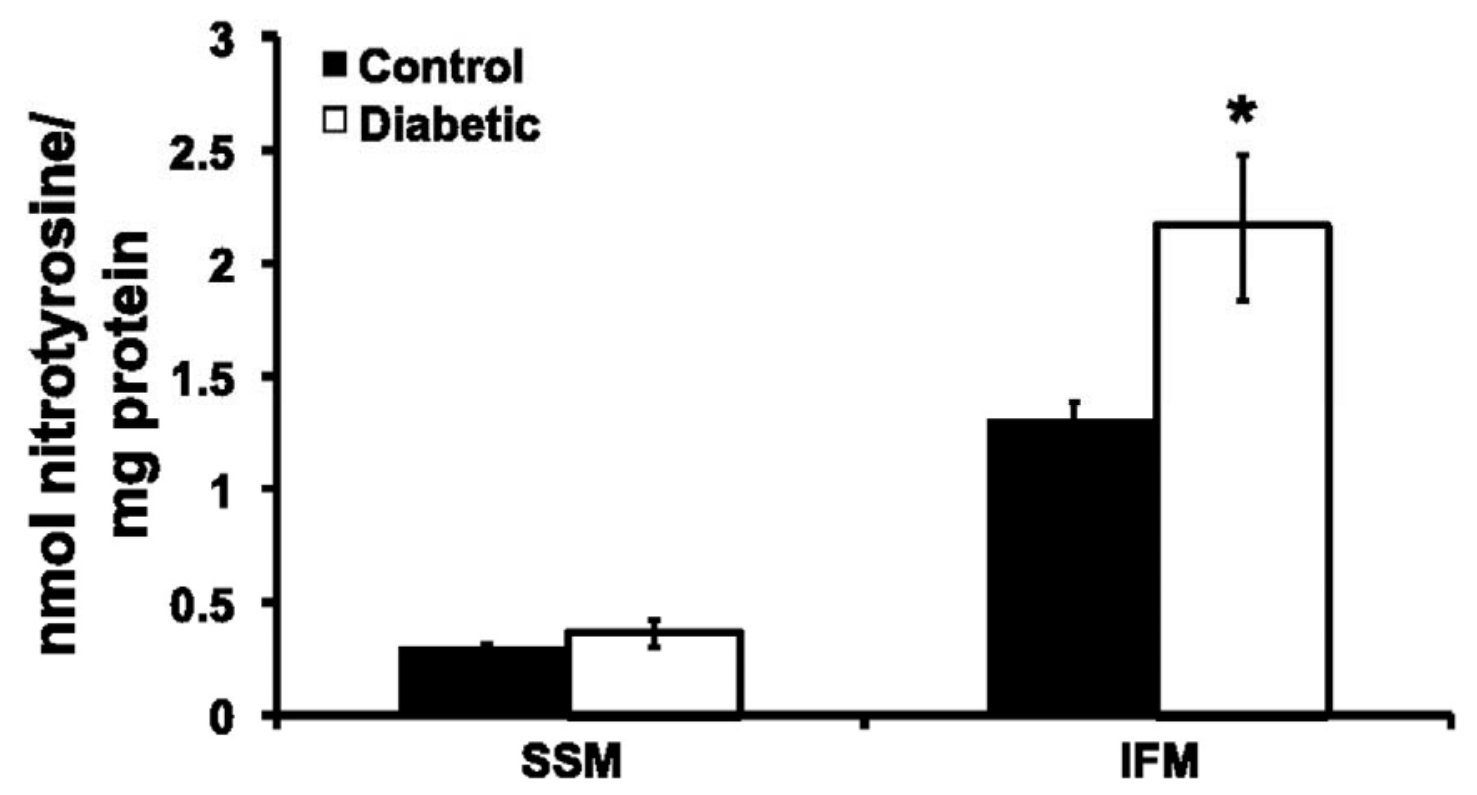


Figure 2.4. Mitochondrial subpopulation protein nitrotyrosine content. Individual mitochondrial subpopulations were isolated from diabetic and control hearts. Assessment of protein nitrotyrosine residues was performed using a sandwich ELISA and results compared against a known protein nitrotyrosine standard curve. Results are expressed as nmol of nitrotyrosine protein per $\mathrm{mg}$ of protein. Values are expressed as mean \pm SEM; $n=5$ for each group. ${ }^{*} P<0.05$ for diabetic vs. control. 
Figure 2.5

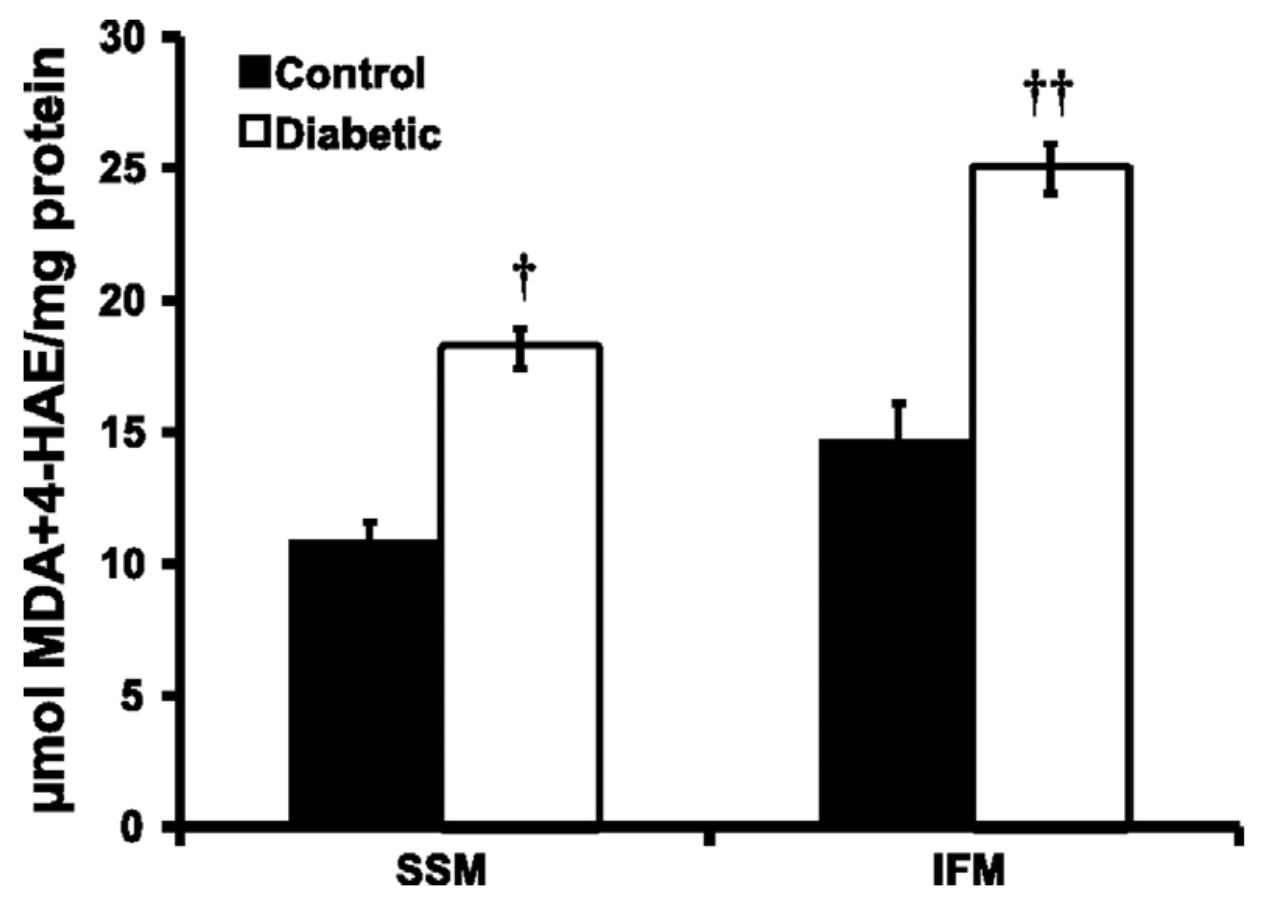


Figure 2.5. Mitochondrial subpopulation lipid peroxidation content. Individual mitochondrial subpopulations were isolated from diabetic and control hearts. Assessment of the lipid peroxidation by-products malondialdehyde (MDA) and 4-hydroxyalkenals (4-HAE) was performed using a colorimetric assay and spectrophotometric measurement at 586 $\mathrm{nm}$. Sample values were compared against a standard curve of known 4HAE and MDA, and data are expressed as $\mu$ mole of MDA+4-HAE per mg of protein. Values are expressed as mean \pm SEM; $n=5$ for each group. $\dagger P<0.01$ for diabetic vs. control. $\dagger \dagger P<0.005$ for diabetic vs. control. 
Figure 2.6

A

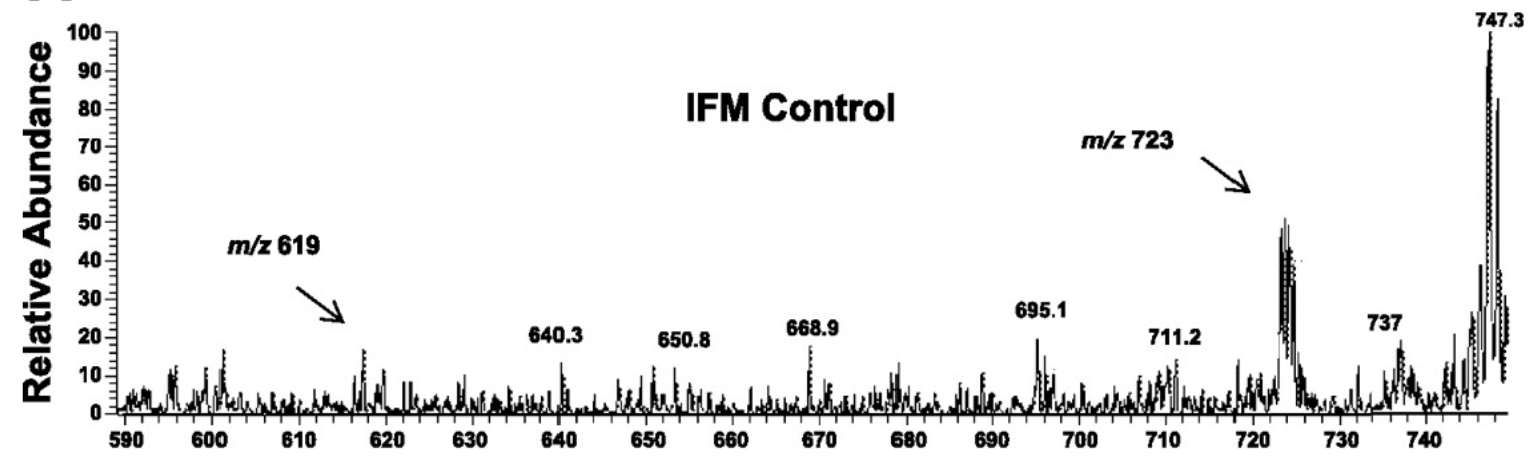

B
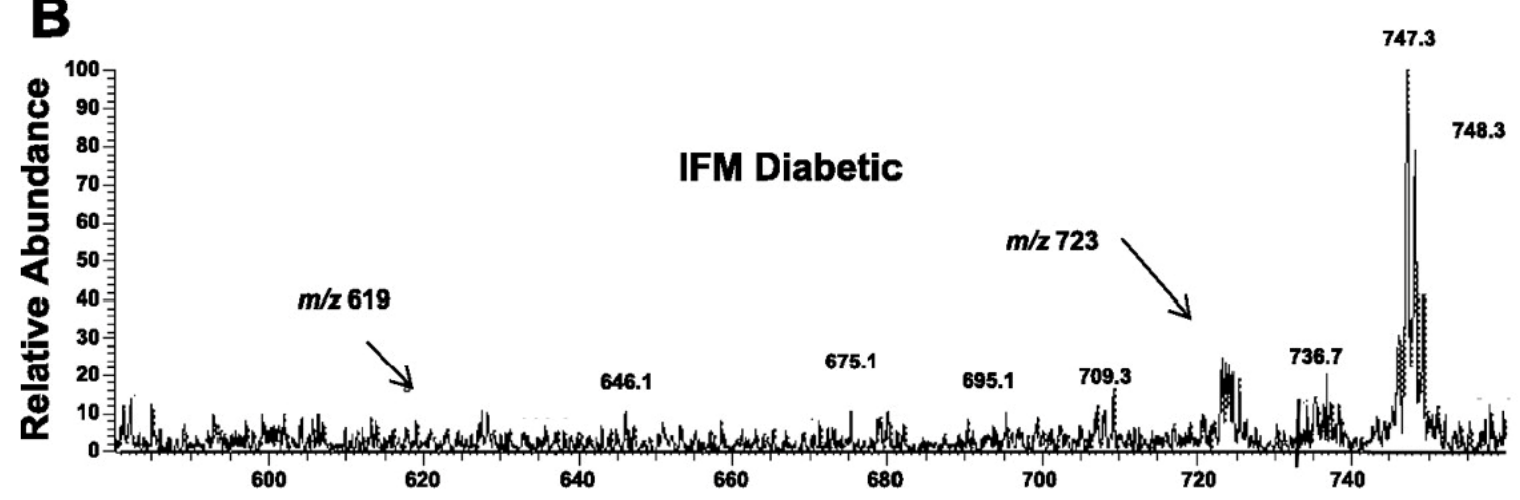
Figure 2.6

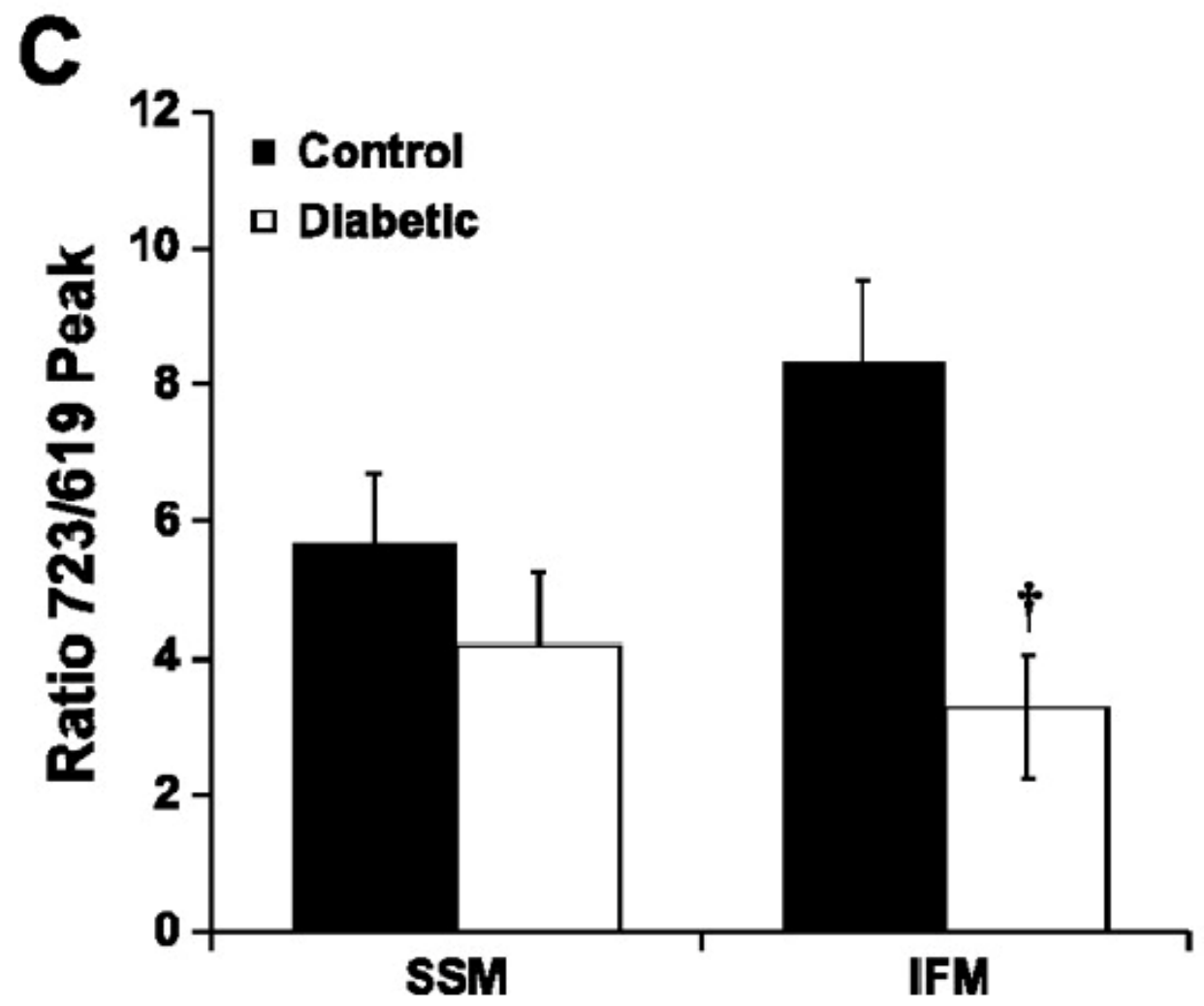


Figure 2.6. Mitochondrial subpopulation cardiolipin content. Individual mitochondrial subpopulations were isolated from diabetic and control hearts, and lipids extracted. Assessment of cardiolipin content was performed by electrospray ionization mass spectrometry (ESI-MS) on diluted samples from control and diabetic mitochondrial subpopulations. The ions of the predominant molecular species of cardiolipin (T18:2 CL, $\mathrm{m} / \mathrm{z} 723)$ and the internal standard (T14:0 CL, $\mathrm{m} / \mathrm{z}$ 619) are indicated by arrows. (A) Representative negative-ion ESI-MS spectra from control SSM. (B) Representative negative-ion ESI-MS spectra from diabetic SSM. (C) Representative negative-ion ESI-MS spectra from control IFM. Representative negative-ion ESI-MS spectra from diabetic IFM. Summary data of cardiolipin expressed as the ratio of $\mathrm{m} / \mathrm{z}$ 723: $\mathrm{m} / \mathrm{z} 619$. Values are expressed as mean $\pm S E M ; n=3$ for each group. $\dagger P<0.01$ for diabetic vs. control. 


\section{Table 2.1}

\begin{tabular}{|c|c|c|c|c|c|c|c|c|}
\hline Group & $\begin{array}{c}\text { Body } \\
\text { Weight, g }\end{array}$ & $\begin{array}{c}\text { Heart } \\
\text { Weight, } \mathbf{~ m g}\end{array}$ & $\begin{array}{c}\text { HW/BW } \\
\text { Ratio, mg/g }\end{array}$ & $\begin{array}{c}+\mathbf{d P} / \mathbf{d t}, \\
\mathbf{m m H g} / \mathbf{s}\end{array}$ & $\begin{array}{c}\mathbf{- d P / d} \text { t, } \\
\mathbf{m m H g} / \mathbf{s}\end{array}$ & $\begin{array}{c}\text { Developed } \\
\text { Pressure, } \\
\mathbf{m m H g}\end{array}$ & $\begin{array}{c}\text { SSM } \\
\text { Protein, } \\
\mathbf{m g} / \mathbf{g} \text { tissue }\end{array}$ & $\begin{array}{c}\text { IFM } \\
\text { Protein, } \\
\mathbf{m g} / \mathbf{g} \text { tissue }\end{array}$ \\
\hline Control & $27.4 \pm 1.3$ & $102 \pm 4$ & $3.7 \pm 0.1$ & $3,398 \pm 128$ & $2,310 \pm 114$ & $96 \pm 2$ & $18.9 \pm 2.1$ & $10.3 \pm 0.8$ \\
\hline Diabetic & $28.4 \pm 0.8$ & $108 \pm 6$ & $3.8 \pm 0.2$ & $1,934 \pm 116^{*}$ & $1,680 \pm 144^{*}$ & $57 \pm 3^{*}$ & $19.5 \pm 2.2$ & $9.5 \pm 1.5$ \\
\hline
\end{tabular}


Table 2.1. Heart weight, body weight, contractile function, and mitochondrial yields. Heart weight (HW), body weight (BW), and heart weight/body weight ratios (HW/BW) five weeks following STZ-treatment (Diabetic) or citrate saline injection (Control). HW is expressed in milligrams (mg), BW is expressed in grams (g), and HW/BW is expressed as $\mathrm{mg} / \mathrm{g}$. Functional measurements were performed in Langendorffperfused hearts, and are expressed as rate of contraction $(+\mathrm{dP} / \mathrm{dt})$, rate of relaxation (-dP/dt), and developed pressure (DP). $+\mathrm{dP} / \mathrm{dt}$ and $-\mathrm{dP} / \mathrm{dt}$ are expressed in $\mathrm{mm} \mathrm{Hg} / \mathrm{sec}$, and DP is expressed in $\mathrm{mm} \mathrm{Hg}$. Individual mitochondrial subpopulation protein yields expressed in $\mathrm{mg} / \mathrm{g}$ wet tissue. Values are expressed as mean $\pm \mathrm{SEM} ; \mathrm{n}=8$ for each group. ${ }^{*} P<0.05$ for diabetic vs. control. 


\section{Chapter 3:}

\section{Proteomic Alterations of Distinct Mitochondrial Subpopulations in the Type 1 Diabetic Heart: Contribution of Protein Import Dysfunction}

Erinne R. Dabkowski ${ }^{1,2}$, Walter A. Baseler ${ }^{1,2}$, Courtney L. Williamson ${ }^{1,2}$, Matthew J. Powell $^{3}$, Trust T. Razunguzwa ${ }^{3}$, and John M. Hollander ${ }^{1,2}$

\footnotetext{
${ }^{1}$ West Virginia University School of Medicine, Division of Exercise Physiology; ${ }^{2}$ Center for Cardiovascular and Respiratory Sciences; Morgantown, WV 26506; ${ }^{3}$ Protea Biosciences Inc.; Morgantown, WV 26507
}

Running Title: Mitochondrial Subpopulations Proteomics and the Type 1 Diabetic Heart 


\begin{abstract}
Diabetic cardiomyopathy is associated with increased risk of heart failure in type 1 diabetic patients. Mitochondrial dysfunction is suggested as an underlying contributor to diabetic cardiomyopathy. Cardiac mitochondria are characterized by subcellular spatial locale including mitochondria located beneath the sarcolemma, subsarcolemmal mitochondria (SSM), and mitochondria situated between the myofibrils, interfibrillar mitochondria (IFM). The goal of this study was to determine whether type 1 diabetic insult in the heart influences proteomic make-up of spatially-distinct mitochondrial subpopulations and to evaluate the role of nuclear-encoded mitochondrial protein import. Utilizing multiple proteomic approaches (iTRAQ, and 2D-DIGE), IFM proteomic make-up was impacted by type 1 diabetes mellitus to a greater extent than SSM as evidenced by decreased abundance of fatty acid oxidation and electron transport chain proteins. Mitochondrial phosphate carrier and adenine nucleotide translocator, inner membrane translocases, were decreased in the diabetic IFM ( $p<0.05$ for both). Mitofilin, a protein involved in mitochondrial morphology, was diminished in the diabetic IFM $(p<0.05)$. Post-translational modifications, included oxidations and deamidations, were most prevalent in the diabetic IFM. Glucose-regulated protein 75 (Grp75), a mitochondrial protein import constituent, was decreased in diabetic IFM $(p<0.05)$. Mitochondrial protein import was decreased in the diabetic IFM with no change in the diabetic SSM $(p<0.05)$. Taken together, these results indicate that mitochondrial proteomic alterations in the type 1 diabetic heart are more pronounced in the IFM. Further, proteomic alterations are associated with nuclear-encoded mitochondrial protein import dysfunction and loss of an essential mitochondrial import constituent, Grp75, implicating this process in the pathogenesis of diabetic cardiomyopathy.
\end{abstract}

Diabetes, Mitochondria, Proteomics 


\section{INTRODUCTION}

Cardiovascular complications, including diabetic cardiomyopathy, are the leading cause of mortality among type 1 diabetic patients. A number of studies have indicated that mitochondrial dysfunction underlies and is a central contributor to the dysfunction seen in the type 1 diabetic heart $(10,12,33,36)$. An increasing amount of evidence suggests that mitochondrial subcellular location influences the pathogenesis of dysfunctional mitochondria in the type 1 diabetic heart $(10,23)$. Cardiac mitochondria are characterized by different spatial location within the cell including mitochondria located at the sarcolemma, subsarcolemmal mitochondria (SSM), and mitochondria situated between the myofibrils, interfibrillar mitochondria (IFM). We and others have shown that these two spatially distinct subpopulations of mitochondria are differentially affected with various pathological insults $(10,23,25,26,30,34,40)$. Specifically, we have demonstrated that the IFM display greater dysfunctional profiles with type 1 diabetic insult, as evidenced by enhanced oxidative stress, diminished ETC function and decreased cardiolipin content.

Proteomic evaluations have played an important role in furthering our understanding of mitochondrial dysfunction in the diabetic heart. Several proteomic studies have been undertaken in various diabetic models in an effort to clarify the nature of the proteomic changes associated with the diabetic heart $(5,14,20,36,38)$. Turko et al. identified 30 altered mitochondrial proteins in isolated mitochondria from STZinduced type 1 diabetic rat hearts (38). In particular, they observed enhanced fatty acid oxidation (FAO) proteins and reduction of several oxidative phosphorylation (OXPHOS) protein subunits. Utilizing the ITRAQ $^{\mathrm{TM}}$ labeling method, Jullig et al. identified 65 proteins significantly changing in the STZ-induced type 1 diabetic rat heart as compared to control (20). The most significant changes were increased levels of FAO enzymes and decreased proteins involved in OXPHOS. In a recent study using label free proteome expression analyses, Bugger et al. examined mitochondrial proteomes of several tissues from the Akita mouse (kidney, liver, brain and heart) (5). Their results indicate that FAO proteins were less abundant in liver mitochondria, whereas FAO 
protein content was induced in mitochondria from all other tissues. In addition, levels of OXPHOS subunits were coordinately increased in liver mitochondria, whereas mitochondria from other tissues were unaffected (5). Taken together, these data suggest that the cardiac mitochondrial proteome is impacted during type 1 diabetes mellitus, though a number of variables may contribute to conflicting results. To date no one has examined proteomic differences in spatially distinct subpopulations of mitochondria in the type 1 diabetic heart which may offer further insight into the nature of this dynamic process in the type 1 diabetic heart.

Studies examining mechanisms underlying or contributing to the proteomic alterations in the diabetic heart are limited (15). Potential mechanisms of proteomic dysregulation may include pathological alterations in gene expression, increases in post-translational modifications (PTMs) of proteins, or upregulation of post-translational regulators such as microRNAs (miRNAs). Alternatively, dysfunctional nuclear-encoded mitochondrial protein import could also disturb the proteomic composition of the mitochondrion. Currently, there are approximately 1200 known proteins in the human mitochondrion (35), with only 13 transcribed and translated inside the organelle itself. As a result, the vast majority of proteins $(\sim 99 \%)$ must be imported into mitochondrion through a complex mechanism of translocation involving interaction between protein targeting signals and mitochondrial translocases (6). To date, no study has examined the impact of type 1 diabetes mellitus on spatially distinct mitochondrial proteomes in the diabetic heart or offered insight into the mechanisms influencing specific proteomic profiles. The goal of this study was to determine whether proteomic differences exist in subpopulations of mitochondria as well as to evaluate the role of nuclear-encoded mitochondrial protein import in the type 1 diabetic heart. 


\section{MATERIALS AND METHODS}

\section{Experimental Animals and Diabetes Induction}

The animal experiments in this study conformed to National Institutes of Health (NIH) Guidelines for the Care and Use of Laboratory Animals and were approved by the West Virginia University Animal Care and Use Committee. Male FVB mice (Charles River Laboratories, Wilmington, MA) were housed in the West Virginia University Health Sciences Center animal facility. Mice were given unlimited access to a rodent diet and water. Type 1 diabetes mellitus was induced in 8-wk-old mice following the protocol of the Animal Models of Diabetic Complications Consortium using multiple low-dose streptozotocin (STZ; Sigma, St. Louis, MO) injections. Injections of $50 \mathrm{mg} / \mathrm{kg}$ body weight STZ dissolved in sodium citrate buffer $(\mathrm{pH} 4.5)$ were performed daily for 5 consecutive days after 6 hours of fasting. Mice that served as vehicle controls were given the same volume per body weight of sodium citrate buffer. One week postinjection, hyperglycemia was confirmed by measuring urinary glucose (Chemstrip 2GP Urine test strips, Roche Diagnostics, Indianapolis, IN), where $>2,000 \mathrm{mg} / \mathrm{dl}$ was considered diabetic. Five weeks post hyperglycemia onset, animals were sacrificed for further experimentation.

\section{Preparation of Individual Mitochondrial Subpopulations}

At 5 weeks post hyperglycemia onset, FVB mice and their littermate controls were sacrificed, and the hearts were excised. Hearts were rinsed in phosphate buffered saline (PBS, pH 7.4), then blotted dry and weighed. SSM and IFM were isolated as previously described following the methods of Palmer et al. (29) with minor modifications $(10,11,40)$. Protein concentrations were determined using the Bradford method with bovine serum albumin (BSA) as a standard (3). 


\section{ITRAQ Labeling}

Pooled SSM and IFM subpopulations $(n=4)$ from diabetic and control hearts were lysed and precipitated overnight in acetone at $-20^{\circ} \mathrm{C}$ and pellets were resuspended in $20 \mu \mathrm{L}$ of $0.5 \mathrm{M}$ triethylammonium bicarbonate (TEAB; $\mathrm{pH}$ 8.5) as previously described (9). Protein contents were determined using a 2-D Quant Kit (Amersham, Piscataway, $\mathrm{NJ}$ ) and $100 \mu \mathrm{g}$ of each pooled sample was than denatured with $0.1 \%$ SDS and reduced with $5 \mathrm{mM}$ tris-(2-carboxyethyl) phosphine (TCEP). After incubation for 1 hour at $60^{\circ} \mathrm{C}$, cysteines were blocked with $10 \mathrm{mM}$ methyl methane thiosulfonate (MMTS) in isopropanol, and the samples were incubated at room temperature for 10 minutes. Addition of $10 \mu \mathrm{L}$ of sequencing grade trypsin (Applied Biosystems, Foster City, CA) was added in a trypsin/protein ratio of $1: 20$, and the samples incubated at $37^{\circ} \mathrm{C}$ overnight. Digested samples were labeled with the ITRAQ ${ }^{\mathrm{TM}}$ reagents following the protocol provided by the vendor (Applied Biosystems, Foster City, CA).

After digestion and iTRAQ labeling, the ultra-complex protein digests were combined to create a $400 \mu \mathrm{g}$ pooled protein digest sample that contained equal fractions of each of the four labeled samples for subsequent Multi Dimensional Protein Identification Technology (MudPIT) analysis (27). After lyophilization, the digest mixture was reconstituted in strong cation exchange (SCX) loading buffer $(5 \mathrm{mM}$ ammonium formate in $20 \%$ acetonitrile; $\mathrm{pH} 3.0$ ) to be fractionated with SCX SpinTips (Protea Biosciences, Morgantown, WV) per the manufacturer's protocol. Briefly, the sample solution was loaded centrifugally onto the SCX SpinTip. The non-adsorbing solution that passed through the SCX SpinTip was collected. Eight different elution solutions were used to fractionate the peptides $(20,60,100,150,200,250,400$, and $500 \mathrm{mM}$ ammonium formate in $10 \%$ acetonitrile) in a step-wise manner, for a total of nine sample fractions. The collected fractions were cleaned by repeated lyophilization and reconstituted in a $0.1 \mathrm{M}$ acetic acid solution, and then lyophilized to dryness. The fractions were then submitted for LC-MALDI TOF/TOF mass spectral analysis for protein identification, characterization, and differential expression analysis. 


\section{Mass Spectrometry (MS) Analyses with iTRAQ ${ }^{\mathrm{TM}}$ Labeling}

The LC-MALDI mass spectrometry system utilized was an ABI Tempo LC MALDI spotter with Tempo LC MALDI v.2.00.09 data acquisition and processing software. Lyophilized SCX sample fractions were reconstituted in LC aqueous run buffer $(0.1 \%$ trifluoroacetic acid, $2 \%$ acetonitrile) and injected onto a Zorbax $\mathrm{C}_{18}$ chromatographic column, $150 \times 0.3 \mathrm{~mm}$ (Agilent Technologies, Wilmington, DE). The peptides were eluted from the column using an acetonitrile/trifluoroacetic acid gradient (2-72\% acetonitrile in 35 minutes) and spotted directly onto a MALDI plate in 6 second spot fractions. The MALDI spots were analyzed using an ABI 4800 MALDI TOF/TOF analyzer operated with 4000 Series Explorer software. The MS acquisition was in positive ion reflector mode with 400 laser shots per spectrum performed. The 15 strongest precursors per spot were chosen for MS/MS and the MALDI spot was interrogated until at least 4 peaks in the MS/MS, spectra achieved a $S / N \geq 70$.

The resulting MS/MS spectra were analyzed using $A B I$ Protein ProteinPilot software 2.0 (Applied Biosystems, Foster City, CA). The spectral data was searched against the mouse protein database (NCBI nr.fasta database customized to select for all mouse proteins) for identification of the peptides and corresponding proteins. In ProteinPilot, the sample type was selected as ITRAQ 4Plex for retrieval of the isotopic tag information from the mass spectra. After database correlation analysis, the proteins were grouped, scored, and normalized against one of four isotope correction factors. The Pro Group algorithm of ProteinPilot generated a ProtScore that is a cumulative score from each of the peptides used by the algorithm in the protein identification. Protein scores (ProtScore) above 2.0, 1.0, and 0.47 expressed the percent confidence levels of $>99,>90$, and $>66 \%$, respectively. Each peptide match showed the iTRAQ isotopic labels, MMTS labeled cysteines, and other post-translational modifications present as mass spectral shifts identified during the database correlation analysis. Each protein identified also showed the differential protein expression compared against the other iTRAQ $^{\text {TM }}$ labeled samples for relative quantitation. 


\section{D-Differential In-Gel Electrophoresis}

\section{Sample Preparation}

Isolated mitochondrial subpopulations were placed in lysis buffer ( $7 \mathrm{M}$ urea, $2 \mathrm{M}$ thiourea, $30 \mathrm{mM}$ Tris, $5 \mathrm{mM}$ magnesium acetate, 4\% CHAPS, and $58 \mathrm{mM}$ DTT) in a 1:10 ratio. Protein concentration of the samples was determined using a 2-D Quant Kit (Amersham Biosciences, Piscataway, NJ).

2D-Difference In-gel Electrophoresis (2D-DIGE) and Differential-display Proteome Analyses

$100 \mu \mathrm{g}$ of each mitochondria subpopulation from both diabetic and control was lysed with labeling buffer (7 M urea, $2 \mathrm{M}$ thiourea, 4\% CHAPS, $30 \mathrm{mM}$ Tris, $5 \mathrm{mM}$ magnesium acetate). One third of each sample was removed and combined into a separate tube to serve as an internal standard. $50 \mu \mathrm{g}$ of the remaining samples were labeled separately with $400 \mathrm{pmol}$ of Cy3 or Cy 5 NHS-ester minimal labeling reagents for 30 minutes on ice in the dark. The samples were then quenched with $2 \mu$ of $10 \mathrm{mM}$ lysine for 10 minutes on ice in the dark. $50 \mu \mathrm{g}$ of the combined sample was also labeled with $400 \mathrm{pmol}$ of Cy2 and than quenched with $10 \mu \mathrm{l}$ of $10 \mathrm{mM}$ lysine. Individual Cy3- and Cy5-labeled samples were combined with the same amount of the Cy2labeled internal standard. Each gel contained one control and one diabetic sample from individual subpopulations, and the Cy 3 and Cy 5 dyes were alternated to account for dye labeling variability. The samples were separated by standard 2D gel electrophoresis utilizing a manifold-equipped IPGphor first dimension isoelectric focusing unit and $24 \mathrm{~cm}$ 3-10 immobilized $\mathrm{pH}$ gradient (IPG) strips. The initial separation was followed by a second-dimension $12 \%$ SDS-PAGE homogenous on hand-cast gels. The Cy2 (mixed standard), Cy3 (sample A) and Cy5 (sample B) components of each gel were visualized separately at $100 \mu \mathrm{m}$ resolution with mutually-exclusive excitation/emission wavelengths using a Typhoon 9400 Variable Mode Fluorescence Imager (Applied Biosystems, Foster City, CA). A Sypro Ruby protein post-stain (Invitrogen, Carlsbad, $\mathrm{CA}$ ) was used to extract protein from the gels. Gel images were submitted to Ludesi 2D Analysis (Ludesi, Malmo, Sweden, http://www.ludesi.com) for spot detection, matching, and analysis. Control:standard and diabetic:standard normalized volume ratios were 
calculated for each protein on every gel, and the internal standards were used to normalize and compare these ratios across the 10 DIGE gels. This method allowed for the calculation of average abundance changes and Student's T-test p-values for the variance of these ratios for each protein-pair across all 10 individual gels.

\section{Mass Spectrometry and Database Analysis}

Proteins that were changing were excised and digested in gel with trypsin protease and the resulting peptides analyzed using a Micromass MALDI micro MX TOF Spectrometer (Waters, Milford, MA). The resulting peptide mass maps were compared to sequences present in the SWISS-PROT and NCBInr databases to generate statistically significant identifications of proteins using GPS Explorer software (Applied Biosystems, Foster City, CA) running the MASCOT search algorithm. Searches were performed without constraining protein molecular weight or isoelectric point, with complete carbamidomethylation of cysteine, partial oxidation of methionine residues, and one missed cleavage also allowed in the search parameters. Molecular Weight Search (MOWSE) scores from database interrogation above $58(p<0.05)$, number of matched ions, number of matching sequence coverage, and correlation of gel region with predicted MW and pl were considered for each protein identification.

\section{Protein Import}

\section{Plasmid Construction}

The fusion protein pAcGFP1-Mito (Clontech Laboratories, Mountain View, CA) containing the precursor subunit VIII of human cytochrome $\mathrm{C}$ oxidase and the green fluorescent protein from Aequorea coerulescens (AcGFP1) was cloned into pIVEX2.3d (Roche Applied Science, Indianapolis, IN) at restriction sites Not I and blunted Nco I/Nhe I creating pMITOGFP1. The correct sequence was confirmed by dideoxynucleotide sequencing. pMITOGFP1 was grown to a concentration of $500 \mathrm{ng} / \mu \mathrm{l}$ and isolated using mini prep plasmid DNA isolation (Qiagen, Valencia, CA). 
In Vitro Synthesis of Mitochondrial Protein

In vitro transcription and translation of MitoGFP1 was performed using the S30 T7 protein expression system (Promega, Madison, WI) as per the manufacturer's protocol. Additionally, fluorescent labeling of MitoGFP1 was performed using the FluoroTect Green $_{\text {Lys }}$ tRNA in vitro labeling system (Promega, Madison, WI) as per the manufacturer's protocol. MitoGFP1 and fluorescent MitoGFP1 lysates were subsequently used as substrates for the in vitro protein import process.

\section{Mitochondrial Protein Import}

The mitochondrial protein import procedure was performed following the protocol from Stojanovski et al. (37) with modifications. Briefly, $40 \mu \mathrm{g}$ of mitochondria was resuspended in $100 \mu \mathrm{l}$ of import buffer $(250 \mathrm{mM}$ Sucrose, $80 \mathrm{mM} \mathrm{KCl}, 5 \mathrm{mM} \mathrm{MgCl}$, 2 $\mathrm{mM} \mathrm{KH}{ }_{2} \mathrm{PO}_{4}, 10 \mathrm{mM}$ MOP-KOH, pH 7.2) with addition of $2 \mathrm{mM} \mathrm{ATP}$ and $2 \mathrm{mM} \mathrm{NADH.}$ 1-5 $\mu \mathrm{l}$ of bacterial lysate containing MitoGFP1 protein was added and protein import performed at increasing time intervals of 2,5 , and 10 minutes at $25^{\circ} \mathrm{C} .2 \mu \mathrm{M}$ valinomycin was added to stop the import reaction, and samples were centrifuged at $12,000 \times g$ for 5 minutes at $4^{\circ} \mathrm{C}$. The supernatant was discarded and the pellet resuspended in SEM buffer (250 mM Sucrose, 1 mM EDTA, $10 \mathrm{mM}$ MOP-KOH, pH 7.2) containing $25 \mu \mathrm{g} / \mathrm{ml}$ trypsin, on ice. Trypsin was subsequently inhibited by protease inhibitor cocktail (BioVision, Mountain View, CA). Samples were centrifuged again at $12,000 \times \mathrm{g}$ for $5 \mathrm{~min}$ at $4^{\circ} \mathrm{C}$. Pellets were then resuspended in lysis buffer (BioVision, Mountain View, California), subjected to SDS-PAGE, and subsequent Western blot analyses performed. Quantification of blots was performed using Pierce ECL Western Blotting Substrate (Pierce Biotech, Rockford, IL). The primary antibody was an antiGFP monoclonal antibody raised in a mouse host (Clontech Laboratories, Mountain View, CA). The secondary antibody was an anti-mouse IgG horseradish peroxidase conjugate (Sigma, St. Louis, MO). Quantification of chemiluminescent signals were detected using a G:BOX (Syngene, Frederick, MD), and data expressed as arbitrary optical density units. Additionally, visualization of imported fluorescent MitoGFP1 was performed using a Typhoon 8600 (GE Healthcare, Piscataway, NJ) with a 532nm excitation. Control for protein loading was confirmed using Ponceau staining. 


\section{Western Blot Analyses}

SDS polyacrylamide gel electrophoresis (SDS-PAGE) was run on $4-12 \%$ gradient gels as previously described $(24,40)$ with equal amounts of protein loaded for each study treatment. Relative amounts of subpopulation-specific mitochondrial glucose regulated protein 75 (Grp75), translocases of the outer membrane 40 and 20 (TOM 40 and TOM 20), translocases of the inner membrane 23 and 44 (TIM23 and TIM44), were analyzed using the following primary antibodies; anti-Grp75 mouse antibody (Product\# SPA-825; Stressgen, Ann Arbor, MI ), anti-TOM40 goat antibody (Product\# sc-11025; Santa Cruz Biotech, Santa Cruz, CA), anti-TOM 20 mouse antibody (Product \# 612278; BD Biosciences, San Jose, CA), anti-TIM23 mouse antibody (Product \# 611222; BD Biosciences, San Jose, CA), and anti-TIM44 mouse antibody (Product \# 612582, BD Biosciences, San Jose, CA). The secondary antibodies used in the analyses were donkey anti-goat IgG HRP conjugate (Product \#sc-2020; Santa Cruz Biotech, Santa Cruz, CA) for TOM40, and goat-anti mouse conjugate (Product\# 31430; Pierce Biotech, Rockford, IL) for Grp75, TOM20, TIM23 and TIM44. Detection of signal was performed according to the manufacturer's instructions as above. Autoradiographic signals and data analysis were performed as above. Control for protein loading was confirmed using Ponceau staining.

\section{Statistics}

Means and standard errors (SEM) were calculated for all data sets. Data were analyzed with a one-way analysis of variance (ANOVA) method to evaluate the main treatment effect, diabetes induction (GraphPad Software Inc., La Jolla, CA). Fisher's Least Significant Difference (LSD) post hoc tests were performed to determine the significant differences among means. When appropriate a Student's T-test was employed. $p<0.05$ was considered significant. 


\section{RESULTS}

\section{Proteomic Analyses}

To determine whether type 1 diabetes mellitus differentially impacts spatially distinct mitochondrial subpopulation proteomes, we utilized both ITRAQ and 2D-DIGE comparative approaches. ITRAQ enables simultaneous identification and relative quantification of mitochondrial proteins through isobaric peptide tagging, while 2D-DIGE analysis is a quantitative technique that enables multiple protein samples to be separated on the same 2-D gel, thereby greatly reducing the introduction of potential artifacts due to gel-to-gel variations. Figure 3.1 is an example of an MS/MS fragment ion (spectra) for a peptide that was used to identify a protein, Grp75. Located below the spectra is an example of an iTRAQ tag mass spectral signature $(\mathrm{m} / \mathrm{z} 114,115,116$, and 117) used to make a relative quantification of Grp75 within the four labeled sample groups (see Figure 3.1 legend for groups). Figures 2A-D are representative 2D gels from four mitochondrial samples, (A) control IFM, (B) diabetic IFM, (C) control SSM, and (D) diabetic SSM, labeled with different Cy dyes. In this example, an individual spot, identified as Grp75, is indicated by the red arrows, and the quantitative differences assessed by examination of peak densities which are shown in each individual representative gel image (Figures 3.2A-D). Grp75 peak density was significantly decreased $(p<0.02)$ in the diabetic IFM as compared to control IFM, with no changes in diabetic SSM as compared to control SSM.

Proteins of Fatty Acid Oxidation (FAO). FAO proteins were significantly altered in both diabetic SSM and diabetic IFM as compared to control using both ITRAQ and 2D-DIGE methods. In contrast to our previous study in a type 2 diabetes mellitus model (9), ITRAQ analysis revealed 8 of 10 proteins involved in FAO which were decreased in the diabetic IFM, while 4 of 10 were decreased in the diabetic SSM (Table 3.1). Interestingly, carnitine palmitoyltransferase-1 (CPT-1), an outer mitochondrial membrane protein that mediates transport of long-chain fatty acids across the membrane, was significantly increased in the diabetic SSM while diminished in the diabetic IFM (Table 3.1). Further, 2D-DIGE analysis identified 6 FAO proteins that were also significantly decreased in the diabetic IFM, while 2 of 6 were decreased in the 
diabetic SSM (Table 3.2). Taken together, both proteomic analyses suggest that FAO proteins are impaired in both diabetic SSM and IFM, with the effect being greatest in the IFM.

Proteins of the Mitochondrial Respiratory Chain. Utilizing both iTRAQ and 2D-DIGE, a number of proteins of the mitochondrial respiratory chain were significantly altered in both diabetic SSM and diabetic IFM as compared to control. With ITRAQ, 9 of 23 respiratory chain proteins identified were significantly decreased in the diabetic IFM as compared to control, while 1 of 23 respiratory chain proteins identified were decreased in expression in the diabetic SSM as compared to control (Table 3.1). Interestingly, 5 of 23 respiratory chain proteins identified were increased in the SSM as compared to control. In the diabetic IFM, all 4 proteins identified from complex $V$ (ATP synthase) were decreased, while 2 of 6 proteins from complex I, and 1 of 4 were from complex IV were decreased (Table 3.1). In the diabetic SSM, one protein each from complexes I, III, IV and V, respectively, were significantly increased as compared to control SSM (Table 3.1). Similar to the ITRAQ analysis, 2D-DIGE analysis revealed 16 proteins of the respiratory chain decreased in the diabetic IFM, with 3 proteins increased in the diabetic SSM (Tables 3.1 and 3.2). Proteins that were decreased in the diabetic IFM were from all respiratory complexes including complexes I, II, III, IV and V with the greatest number of decreased proteins occurring at complex I. Similar to the ITRAQ analysis, several respiratory proteins were increased in the diabetic SSM suggesting a possible compensatory mechanism to prevent dysfunction.

Proteins of the Citric Acid Cycle (TCA). ITRAQ and 2D-DIGE analyses of proteins involved in the TCA revealed significant changes in both the diabetic SSM and IFM as compared to control. In the diabetic IFM, 2 of 5 TCA proteins were decreased, while 3 of 5 proteins were increased in the diabetic SSM (Table 1). Of particular interest was the observation that isocitrate dehydrogenase $2(\mathrm{NADP}+)$ displayed increased content in the diabetic SSM yet decreased content in the diabetic IFM (Table 3.1). Similar to the ITRAQ analysis, 2D-DIGE analysis reported 6 TCA proteins decreased in the diabetic IFM, and 3 proteins increased in the diabetic SSM (Table 2). These data suggest again 
that TCA proteins and other proteins necessary for energy production are increased in the diabetic SSM, yet decreased in the diabetic IFM.

Transport Proteins. Proteins involved in transport of proteins and or substrates across the IMM were preferentially decreased in the diabetic IFM as compared to control IFM as evidenced by a decreased content in 3 of 4 proteins, with no changes in the SSM (Table 3.1). Mitochondrial phosphate carrier, a protein that is involved in transport of phosphate groups from the cytosol to the mitochondrial matrix, was significantly decreased in diabetic IFM as compared to control (Table 3.1). Another translocase required for exchange of ADP and ATP across the IMM, adenine nucleotide translocator 1 (ANT1), was also significantly decreased in the diabetic IFM as compared to control (Table 3.1). Interestingly, Grp75, a heat shock protein and a constituent of the mitochondrial import process essential for import of proteins into mitochondrion, was also significantly decreased only in diabetic IFM as compared to control (Tables 3.1 and 3.2) . Western blot analyses of Grp75 (Figure 3.5C), provided confirmation of both the ITRAQ and 2D-DIGE results observed. Another heat shock protein involved in folding and protein import into the mitochondrion, Hsp60, was also significantly decreased in only the diabetic IFM as revealed by 2D-DIGE analysis (Table 3.2).

Structural Proteins. With both ITRAQ and 2D-DIGE analyses, alterations to proteins involved in mitochondrial structure were apparent in only the diabetic IFM as compared to control with no changes in diabetic SSM. In particular, mitofilin, a transmembrane IMM protein that regulates mitochondrial morphology (cristae density) and mitochondrial fusion and fission (18), was significantly decreased only in diabetic IFM as indicated by both ITRAQ and 2D-DIGE analysis (Tables 3.1 and 3.2). Further, prohibitin-2, a protein involved in mitochondrial morphology as well as maintenance of mitochondrial DNA (1), was significantly decreased in the diabetic IFM with 2D-DIGE analysis (Table 3.2). These data are consistent with our previously published data suggesting that mitochondrial internal complexity is decreased in the diabetic IFM (10).

Post-translational Modifications (PTM) 
Utilizing MudPIT technology to assess PTM, 42 proteins were identified as having a PTM in the diabetic IFM while 23 proteins were identified in the diabetic SSM (Table 3.3). Interestingly, 11 of the 42 proteins in the diabetic IFM contained oxidations on various amino acid residues. In particular, 3 proteins of complex III, 2 proteins of complex $\mathrm{V}$ and 1 protein of complex I were oxidized in the diabetic IFM. Further, Grp75 and mitofilin were also only oxidized in the diabetic IFM. Additionally, 13 proteins in the diabetic IFM and 8 proteins in the diabetic SSM involved in the ETC presented with some sort of PTM including acetylations, deamidations and methylations. Of greatest interest was that the majority of the modified proteins were IMM proteins, suggesting that this locus may be specifically impacted and as a result, play a key role in the pathogenesis of cardiac dysfunction associated with type 1 diabetes mellitus.

\section{Mitochondrial Protein import Construct}

Figure 3.3A is a vector map showing the insertion of MITOGFP1 cDNA that was cut Not I, and blunted at Nhe I. The cDNA was inserted as a Not I, Nhe I blunt fragment into the PIVEX2.3d plasmid. PIVEX2.3d has all necessary components for efficient protein production in a cell free bacterial protein expression lysate system including a T7 promoter, ribosomal binding site, and poly A tail. The MITOGFP1 gene consists of an $\mathrm{N}$-terminal mitochondrial targeting sequence derived from cytochrome $\mathrm{C}$ oxidase subunit VIII (targeted to the matrix) fused with AcGFP. Translated MitoGFP1 protein is incubated with isolated mitochondria to allow for proper import. Mitochondria are subsequently cleaned of non-imported protein, lysed, and a Western blot analysis is performed probing for AcGFP. Isolated cardiac mitochondria alone does not produce a signal on the blot, (Figure 3.3B, lane 1) whereas pure protein lysate from the bacterial expression system does produce a $31 \mathrm{kDa}$ band (Figure 3.3B, lane 2). Addition of total mitochondria and lysate together produce a similar $31 \mathrm{kDa}$ band, which is the full length precursor protein yet to be completely imported, and a $27 \mathrm{kDa}$ band that represents the mature MitoGFP1 protein in which the precursor sequence has been cleaved and properly imported into the mitochondrial matrix (Figure 3.3B, lane 3). 


\section{Mitochondrial Protein Import}

Utilizing Western blot analysis, Figures 3.4A and $\mathrm{C}$ show increasing amounts of protein abundance at 2,5 , and 10 minutes, respectively, of the mature $27 \mathrm{kDa}$ MitoGFP1 in the control SSM and diabetic SSM subpopulations with no statistical

difference between the samples. Conversely, a significant decrease $(40 \%)$ in the amount of MitoGFP1 imported into the diabetic IFM compared to control IFM is shown at all three time points (Fig 3.4B and D). Additionally, fluorescent labeling and subsequent import of MitoGFP1 was performed to confirm our Western blot findings (3.4A and $B$ ). The $31 \mathrm{kDa}$ band is also decreased in the diabetic IFM compared to control denoting lesser amounts of precursor proteins in transit to the matrix.

\section{Protein Import Machinery}

Outer and inner mitochondrial membrane translocases essential for proper mitochondrial import were also analyzed by Western blot analysis. There were no significant differences observed in the protein expression of TOM20, TOM40, TIM23, or TIM44 in either the diabetic SSM or diabetic IFM as compared to control (Fig 3.5A). Contrary to these results, the essential mitochondrial protein import motor Grp75 was significantly decreased only in the diabetic IFM subpopulation compared to control (Fig 3.5C) with no change in the diabetic SSM compared to control (Fig 3.5B). These results indicate that of the major mitochondrial protein import constituents examined only Grp75 is decreased in abundance during type 1 diabetic insult, and this phenomenon is specific to the IFM subpopulation. 


\section{DISCUSSION}

A significant amount of data supports the notion that mitochondrial dysfunction plays a critical role in the pathogenesis of diabetic cardiomyopathy. Despite the growing evidence for mitochondrial abnormalities underlying diabetic cardiomyopathy, the mechanisms involved in this dysfunction are not entirely clear. Evidence suggests that mitochondrial dysfunction varies depending on subcellular location, with those mitochondria located between the myofibrils being affected to a greater extent in the type 1 diabetic heart $(10,40)$. Data also suggests that dysfunction manifests via disturbance to the IMM as evidenced by diminished ETC complex activities, an enhanced oxidative environment and depletion of cardiolipin, an essential phospholipid required for proper mitochondrial function (31). The goal of this study was to build upon our previous findings and determine whether mitochondrial subpopulation-specific proteomes are altered with type 1 diabetes mellitus in the heart. In an effort to elucidate mechanism, we examined whether mitochondrial import of nuclear-encoded proteins is decreased, resulting in mitochondrial proteome dysregulation.

Proteomic analyses have played an integral role in furthering our understanding of mitochondrial dysfunction in the diabetic heart. Several proteomic studies, in a variety of diabetic models, have been employed to assess proteomic changes associated with the diabetic heart $(5,9,14,20,36,38)$. However, to date no examination of subpopulation-specific proteomic make-up has been performed in a type 1 diabetic model. Proteomic profile comparisons of mitochondrial subpopulations from diabetic and control mouse hearts revealed that the majority of proteins changing were in the IFM and that almost all of these changes were decreases in protein abundances. Utilizing ITRAQ and 2D-DIGE methodologies afforded a number of advantages over the use of a single proteomic methodology including the ability to confirm experimental results and accuracy by essentially comparing results from each method. By using MudPIT analysis combined with ITRAQ labeling, the limitations associated with 2DDIGE, such as solubility and separation, were bypassed. Further, the ITRAQ approach enabled comparison of total protein levels, which can be more difficult using 2D-DIGE separation, where PTMs such as phosphorylation can alter pls and hence gel migration 
(20). Utilizing 2D-DIGE allowed us to examine proteins that may be in less abundance and as a result, less likely to show up with iTRAQ analyses. Because each method has its own unique set of advantages, the combined use of both techniques enhanced our level of confidence in the reported findings.

The majority of proteins identified as changing with both 2D-DIGE and ITRAQ approaches in the diabetic IFM were FAO and ETC proteins. In contrast to previous studies $(20,38)$, proteins involved in FAO (transport and/or utilization of fatty acids) were decreased in abundance in the diabetic IFM to a greater extent than in diabetic SSM. Though only a few fatty acid utilization enzymes were also decreased in diabetic SSM, CPT-1, a transporter of fatty acids into the mitochondrion, was upregulated. One could hypothesize that the diminished ability to utilize fatty acids within the diabetic IFM may have hampered its ability to produce ATP which in turn, contributed to an overall decrease in cardiac contractile function as reported previously (10,39). Additionally, ETC complexes and translocases within the IMM act as key contributors to overall oxidative phosphorylation and energy production within the mitochondrion. In total, we identified 9 ETC proteins from ITRAQ analyses and 16 ETC proteins from 2D-DIGE analyses as being decreased in the diabetic IFM. Jullig et al. reported decreases in ETC constituents that are involved in oxidative phosphorylation in total mitochondria (20), which is somewhat different from our current study examining mitochondrial subpopulations. Further, translocases located within the IMM that are essential for energy production through synthesis (mitochondrial phosphate carrier) and transport of ATP (ANT1) were also significantly decreased only in the diabetic IFM. Interestingly, Jullig et al. did not report changes in ANT1 or the mitochondrial phosphate carrier which may have resulted from their analyses being performed on total mitochondria as opposed to our study examining subpopulations of mitochondria. Our proteomic data are consistent with previous reports of decreased respiratory capacity in the diabetic heart as well as functional data indicating down-regulation of ETC activities, specifically in the IFM $(10,23,36)$. Down-regulation of multiple components of the oxidative phosphorylation machinery and FAO proteins suggests that mitochondria in the type 1 diabetic heart, specifically IFM, may be less efficient in producing ATP required for contraction and calcium homeostasis. 
Mitochondrial morphological abnormalities have been reported with both type 1 and type 2 diabetes mellitus $(4,22,36)$. Previous reports suggest that IFM morphology is specifically impacted by diabetes mellitus $(10,22)$. We reported previously that IFM were smaller and less complex in the type 1 diabetic heart with no changes in the SSM (10). Both 2D-DIGE and ITRAQ analyses indentified mitofilin, a protein critical for proper cristae morphology (18), as being decreased only in the diabetic IFM. It is conceivable that diminished mitofilin, along with our previous reports of depleted cardiolipin may have been a contributing factor to the altered IFM morphology reported previously (10).

To date, no attempts have been made to define the mechanisms accounting for mitochondrial proteomic dysregulation in the diabetic heart. Potential mechanisms of proteomic dysregulation may include alterations in gene expression, enhanced PTMs, or upregulation of regulators such as miRNAs $(21,28)$. In the present study, PTMs identified included oxidations, deamidations, deacetylations and methylations, with oxidations and deamidations being the most prevalent. Interestingly, the diabetic IFM displayed the greatest number of oxidatively-modified proteins. These findings complement previous studies suggesting that enhanced mitochondrial ROS formation during type 1 diabetic insult may precipitate oxidative damage to mitochondrial constituents $(2,10,36)$. Also in agreement are the results from our previous study reporting increased superoxide formation in the type 1 diabetic IFM and the associated enhancement of protein oxidation (nitrotyrosine) within the mitochondrion (10). Another PTM that was prevalent in the diabetic IFM was deamidation. It has been suggested that deamidation of asparaginyl and glutaminyl residues within proteins causes structural and biological alterations to peptide and protein structure (32). Though the contribution of deamidation and oxidation to protein dysfunction is somewhat unclear, these PTMs may alter protein structure/function making the protein more susceptible for degradation by the proteasome system $(16,32)$. In addition, several proteins that were decreased in the diabetic IFM also contained oxidations and deamidations within their structure. Interestingly, Grp75, mitofilin and several components of complex $\vee$ were decreased in abundance with both proteomic technologies and contained oxidations and deamidations within their structures. It is plausible that these modifications may 
have contributed to altered functionality and subsequent proteasomal degradation, all of which may have contributed to the morphological and oxidative phosphorylation deficiencies present in the type 1 diabetic heart.

Given that the majority of mitochondrial proteins are nuclear-encoded and must be imported into the mitochondrion, it is possible that defects in this complicated process may underlie proteomic dysregulation in the diabetic heart. Nuclear-encoded mitochondrial proteins constitute $99 \%$ of the organelle's proteome (6). These proteins are translated in the cytoplasm and must be imported into the mitochondrion via an intricate mechanism involving coordination between the outer and inner mitochondrial membranes (7). The two membranes come together to form a "supercomplex", which creates a singular avenue for translocation of the imported protein into the IMM and matrix (7). Given the vast importance of this process, protein import decrements resulting from pathophysiological conditions could represent a mechanism for mitochondrial dysfunction in the diabetic heart. Hence, a major goal of this study was to gain insight into whether diabetes mellitus causes protein import deficiencies in the mitochondrion and determine whether this phenomenon manifests differently in mitochondrial subpopulations. Utilizing a novel mitochondrial protein import methodology that eliminated the use of protein radiolabeling, we observed dysfunction of the IFM protein import process without effect to the SSM. Previous literature examining mitochondrial protein import in the skeletal muscle of aging mice revealed enhanced cytoplasmic protein degradation, as opposed to protein import decrements as the contributing source of the mitochondrial dysfunction (15). It is unclear why the differences were observed but they may be due in part to the different pathologies and tissue types examined.

Mitochondrial protein import dysfunction has been attributed in part to cardiolipin loss (19). Cardiolipin is known to surround and stabilize protein import complexes including TIM23, the major pore forming unit essential for protein translocation. Studies examining a mutation of cardiolipin, leading to loss of the phospholipid caused reduced membrane potential and decreased mitochondrial protein import (17). Another potential mechanism of mitochondrial import dysfunction is PTMs to proteins essential for protein translocation. As indicated above, we observed multiple modifications to Grp75 in the 
diabetic IFM subpopulation. Grp75 is a heat shock protein and the central subunit of the presequence translocase-associated motor complex (PAM) that binds to a translocating polypeptide chain and drives its movement through the IMM into the matrix by a reaction cycle that requires hydrolysis of ATP (6). Previous literature examining inactivation of yeast Grp75 revealed a near complete inhibition of oxidase assembly 1 protein translocation through the IMM (13). Additionally, cardiac mitochondria in senescent mice were shown to have elevated Grp75 protein content as well as increased protein import of a mitochondrial matrix protein, malate dehydrogenase (8). In our study, we show a marked decrease in Grp75 protein content coinciding with decreased protein import in the diabetic IFM subpopulation, suggesting that Grp75 protein content is proportional to protein import capacity. Our examination of several other key translocases that play a major role in mitochondrial protein import including translocase of the outer membrane 20 (TOM20), outer membrane pore TOM40, inner membrane pore TIM23, and Grp75 docking subunit TIM44, indicated that none of these mitochondrial protein import constituents were decreased in the diabetic heart. Taken together, these results suggest that decreased Grp75 content may underlie IFM protein import deficiencies during type 1 diabetic insult in the heart.

In conclusion, we report here that type 1 diabetic insult differentially influences spatially distinct cardiac mitochondrial proteomes, with the greatest impact occurring to those mitochondria located between the myofibrils (IFM). Further, changes in mitochondrial proteomic make-up are associated with dysfunction to the nuclearencoded mitochondrial protein import machinery and loss of a key mitochondrial protein import constituent, Grp75, implicating this important process in the mechanistic pathogenesis of diabetic cardiomyopathy. 


\section{ACKNOWLEDGEMENTS}

This work was supported by the National Institutes of Health from the National Institutes of Diabetes and Digestive and Kidney Diseases [DP2DK083095]. This work was also supported by a Grant-In-Aid from the American Heart Association [0855484D]. Erinne Dabkowski is a recipient of an American Heart Association Predoctoral Fellowship [0815406D]. Walter Baseler is a recipient of an NIH Predoctoral Fellowship [T32HL090610]. 


\section{REFERENCES}

1. Artal-Sanz M and Tavernarakis N. Prohibitin and mitochondrial biology. Trends Endocrinol Metab 20: 394-401, 2009.

2. Baynes JW. Role of oxidative stress in development of complications in diabetes. Diabetes 40: 405-412, 1991.

3. Bradford MM. A rapid and sensitive method for the quantitation of microgram quantities of protein utilizing the principle of protein-dye binding. Analytical biochemistry 72: 248-254, 1976.

4. Bugger H, Boudina S, Hu XX, Tuinei J, Zaha VG, Theobald HA, Yun UJ, McQueen AP, Wayment B, Litwin SE, and Abel ED. Type 1 diabetic akita mouse hearts are insulin sensitive but manifest structurally abnormal mitochondria that remain coupled despite increased uncoupling protein 3. Diabetes 57: 2924-2932, 2008.

5. Bugger H, Chen D, Riehle C, Soto J, Theobald HA, Hu XX, Ganesan B, Weimer BC, and Abel ED. Tissue-specific remodeling of the mitochondrial proteome in type 1 diabetic akita mice. Diabetes 58: 1986-1997, 2009.

6. Chacinska A, Koehler CM, Milenkovic D, Lithgow $\mathbf{T}$, and Pfanner $\mathbf{N}$. Importing mitochondrial proteins: machineries and mechanisms. Cell 138: 628-644, 2009.

7. Chacinska A, Rehling P, Guiard B, Frazier AE, Schulze-Specking A, Pfanner

$\mathbf{N}$, Voos W, and Meisinger C. Mitochondrial translocation contact sites: separation of dynamic and stabilizing elements in formation of a TOM-TIM-preprotein supercomplex. EMBO J 22: 5370-5381, 2003.

8. Craig EE and Hood DA. Influence of aging on protein import into cardiac mitochondria. Am J Physiol 272: H2983-2988, 1997.

9. Dabkowski ER, Baseler WA, Williamson CL, Powell M, Razunguzwa TT, Frisbee JC, and Hollander JM. Mitochondrial Dysfunction in the Type 2 Diabetic Heart is Associated with Alterations in Spatially-Distinct Mitochondrial Proteomes. Am J Physiol Heart Circ Physiol: ajpheart.00267.02010, 2010. 
10. Dabkowski ER, Williamson CL, Bukowski VC, Chapman RS, Leonard SS, Peer CJ, Callery PS, and Hollander JM. Diabetic cardiomyopathy-associated dysfunction in spatially distinct mitochondrial subpopulations. Am J Physiol Heart Circ Physiol 296: H359-369, 2009.

11. Dabkowski ER, Williamson CL, and Hollander JM. Mitochondria-specific transgenic overexpression of phospholipid hydroperoxide glutathione peroxidase (GPx4) attenuates ischemia/reperfusion-associated cardiac dysfunction. Free Radic Biol Med 45: 855-865, 2008.

12. Flarsheim CE, Grupp IL, and Matlib MA. Mitochondrial dysfunction accompanies diastolic dysfunction in diabetic rat heart. Am J Physiol 271: H192-202, 1996.

13. Frazier AE, Chacinska A, Truscott KN, Guiard B, Pfanner N, and Rehling P. Mitochondria use different mechanisms for transport of multispanning membrane proteins through the intermembrane space. Mol Cell Biol 23: 7818-7828, 2003.

14. Hamblin M, Friedman DB, Hill S, Caprioli RM, Smith HM, and Hill MF. Alterations in the diabetic myocardial proteome coupled with increased myocardial oxidative stress underlies diabetic cardiomyopathy. $\mathrm{J} \mathrm{Mol} \mathrm{Cell} \mathrm{Cardiol} \mathrm{42:} \mathrm{884-895,}$ 2007.

15. Huang JH, Joseph AM, Ljubicic V, Iqbal S, and Hood DA. Effect of age on the processing and import of matrix-destined mitochondrial proteins in skeletal muscle. $J$ Gerontol A Biol Sci Med Sci 65: 138-146, 2010.

16. Iwai K, Drake SK, Wehr NB, Weissman AM, LaVaute T, Minato N, Klausner RD, Levine RL, and Rouault TA. Iron-dependent oxidation, ubiquitination, and degradation of iron regulatory protein 2: implications for degradation of oxidized proteins. Proc Natl Acad Sci U S A 95: 4924-4928, 1998.

17. Jiang $F$, Ryan MT, Schlame M, Zhao M, Gu Z, Klingenberg M, Pfanner N, and Greenberg ML. Absence of cardiolipin in the crd1 null mutant results in decreased mitochondrial membrane potential and reduced mitochondrial function. $\mathrm{J}$ Biol Chem 275: 22387-22394, 2000. 
18. John GB, Shang Y, Li L, Renken C, Mannella CA, Selker JM, Rangell L, Bennett MJ, and Zha J. The mitochondrial inner membrane protein mitofilin controls cristae morphology. Mol Biol Cell 16: 1543-1554, 2005.

19. Joshi AS, Zhou J, Gohil VM, Chen S, and Greenberg ML. Cellular functions of cardiolipin in yeast. Biochim Biophys Acta 1793: 212-218, 2009.

20. Jüllig M, Hickey AJ, Middleditch MJ, Crossman DJ, Lee SC, and Cooper GJS. Characterization of proteomic changes in cardiac mitochondria in streptozotocindiabetic rats using iTRAQ $<$ SUP $><$ FONT SIZE $=$ '-1' $>$ TM $</ F O N T></ S U P>$ isobaric tags. PROTEOMICS - Clinical Applications 1: 565-576, 2007.

21. Kane LA and Van Eyk JE. Post-translational modifications of ATP synthase in the heart: biology and function. J Bioenerg Biomembr 41: 145-150, 2009.

22. Kelley DE, He J, Menshikova EV, and Ritov VB. Dysfunction of mitochondria in human skeletal muscle in type 2 diabetes. Diabetes 51: 2944-2950, 2002.

23. King KL, Young ME, Kerner J, Huang H, O'Shea KM, Alexson SE, Hoppel CL, and Stanley WC. Diabetes or peroxisome proliferator-activated receptor alpha agonist increases mitochondrial thioesterase I activity in heart. J Lipid Res 48: 15111517, 2007.

24. Laemmli UK. Cleavage of structural proteins during the assembly of the head of bacteriophage T4. Nature 227: 680-685, 1970.

25. Lesnefsky EJ, Gudz TI, Moghaddas S, Migita CT, Ikeda-Saito M, Turkaly PJ, and Hoppel CL. Aging decreases electron transport complex III activity in heart interfibrillar mitochondria by alteration of the cytochrome c binding site. $\mathrm{J} \mathrm{Mol} \mathrm{Cell}$ Cardiol 33: 37-47, 2001.

26. Lesnefsky EJ, Slabe TJ, Stoll MS, Minkler PE, and Hoppel CL. Myocardial ischemia selectively depletes cardiolipin in rabbit heart subsarcolemmal mitochondria. Am J Physiol Heart Circ Physiol 280: H2770-2778, 2001.

27. Lin D. Multidimensional Protein Identification Technology as an Effective Tool for Proteomics. American Genomic/Proteomic Technology 1: 38-46, 2001.

28. Nishi H, Ono K, Iwanaga Y, Horie T, Nagao K, Takemura G, Kinoshita M, Kuwabara Y, Mori RT, Hasegawa K, Kita T, and Kimura T. MicroRNA-15b modulates 
cellular ATP levels and degenerates mitochondria via Arl2 in neonatal rat cardiac myocytes. J Biol Chem 285: 4920-4930, 2010.

29. Palmer JW, Tandler B, and Hoppel CL. Biochemical properties of subsarcolemmal and interfibrillar mitochondria isolated from rat cardiac muscle. $\mathrm{J}$ Biol Chem 252: 8731-8739, 1977.

30. Palmer JW, Tandler B, and Hoppel CL. Heterogeneous response of subsarcolemmal heart mitochondria to calcium. Am J Physiol 250: H741-748, 1986.

31. Paradies G, Petrosillo G, Paradies V, and Ruggiero FM. Role of cardiolipin peroxidation and $\mathrm{Ca} 2+$ in mitochondrial dysfunction and disease. Cell Calcium 45: 643650, 2009.

32. Robinson $A B$ and Rudd $C J$. Deamidation of glutaminyl and asparaginyl residues in peptides and proteins. Curr Top Cell Regul 8: 247-295, 1974.

33. Rolo AP and Palmeira CM. Diabetes and mitochondrial function: role of hyperglycemia and oxidative stress. Toxicol Appl Pharmacol 212: 167-178, 2006.

34. Rosca MG, Vazquez EJ, Kerner J, Parland W, Chandler MP, Stanley W, Sabbah HN, and Hoppel CL. Cardiac mitochondria in heart failure: decrease in respirasomes and oxidative phosphorylation. Cardiovasc Res 80: 30-39, 2008.

35. Scharfe C, Lu HH, Neuenburg JK, Allen EA, Li GC, Klopstock T, Cowan TM, Enns GM, and Davis RW. Mapping gene associations in human mitochondria using clinical disease phenotypes. PLoS Comput Biol 5: e1000374, 2009.

36. Shen X, Zheng S, Thongboonkerd V, Xu M, Pierce WM, Jr., Klein JB, and Epstein PN. Cardiac mitochondrial damage and biogenesis in a chronic model of type 1 diabetes. Am J Physiol Endocrinol Metab 287: E896-905, 2004.

37. Stojanovski D, Pfanner N, and Wiedemann N. Import of proteins into mitochondria. Methods Cell Biol 80: 783-806, 2007.

38. Turko IV and Murad F. Quantitative protein profiling in heart mitochondria from diabetic rats. J Biol Chem 278: 35844-35849, 2003.

39. Weytjens C, Cosyns B, D'Hooge J, Droogmans S, Lahoutte T, Garbar C, Roossens B, and Van Camp G. Evaluation of contractile function and inotropic reserve with tissue velocity, strain and strain rate imaging in streptozotocin-induced diabetes. Eur J Echocardiogr, 2010. 
40. Williamson CL, Dabkowski ER, Baseler WA, Croston TL, Alway SE, and Hollander JM. Enhanced apoptotic propensity in diabetic cardiac mitochondria: influence of subcellular spatial location. Am J Physiol Heart Circ Physiol 298: H633-642, 2010. 
Figure 3.1

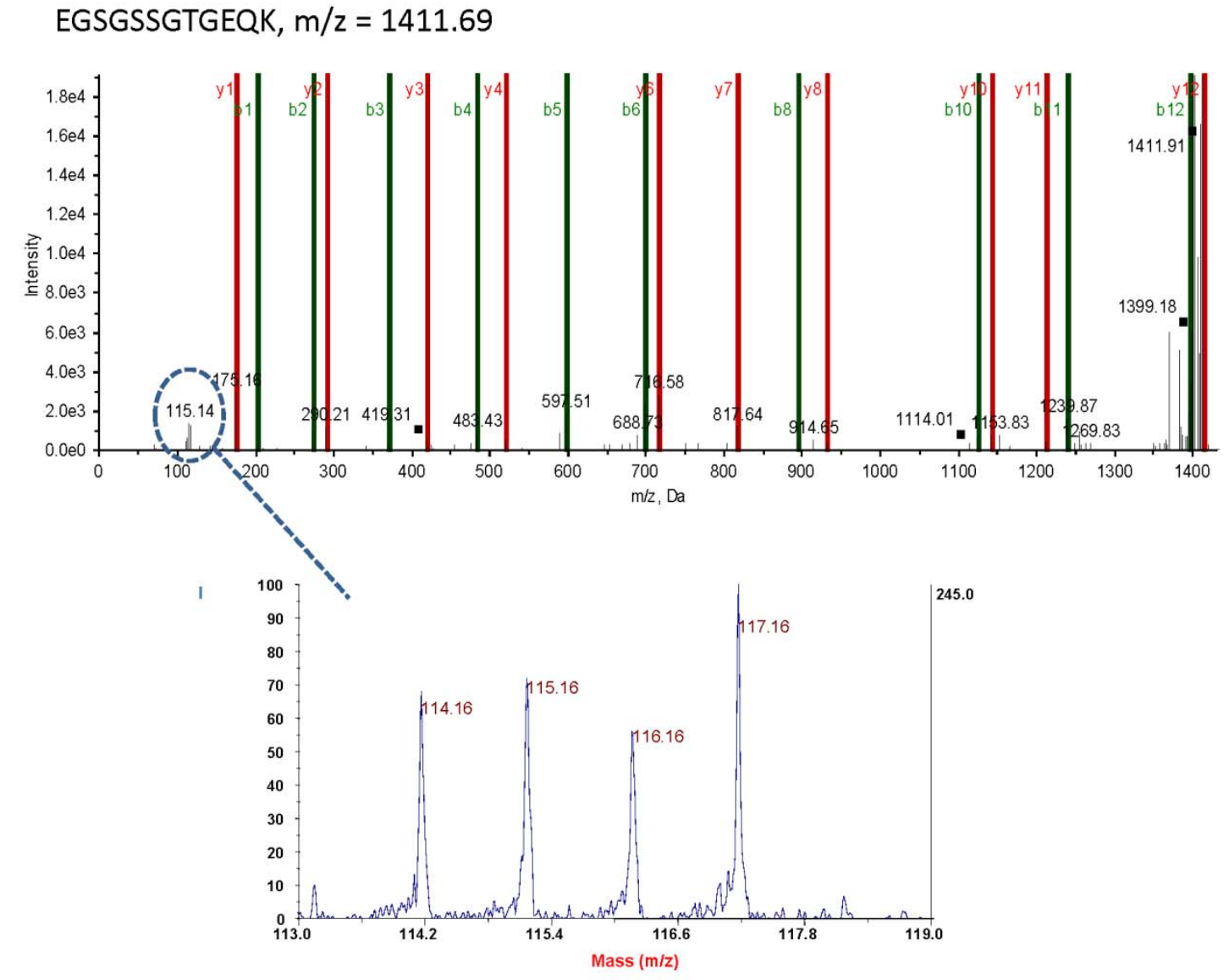


Figure 3.1. Representative ITRAQ spectra. Isolated mitochondrial subpopulations from control and diabetic hearts were labeled with iTRAQ reagents 114 (diabetic SSM), 115 (diabetic IFM), 116 (control SSM), 117 (diabetic IFM) and combined for analysis with mass spectrometry. Representative spectra of simultaneous quantitation of a Grp75 peptide in control and diabetic mitochondrial subpopulations. (B) MS/MS spectra for the reporter groups of the iTRAQ reagents $(114,115,116$ and 117) from a Grp75 peptide. These spectra were used along with other peptides to simultaneously quantify Grp75 control and diabetic mitochondrial subpopulations. 
Figure 3.2

A
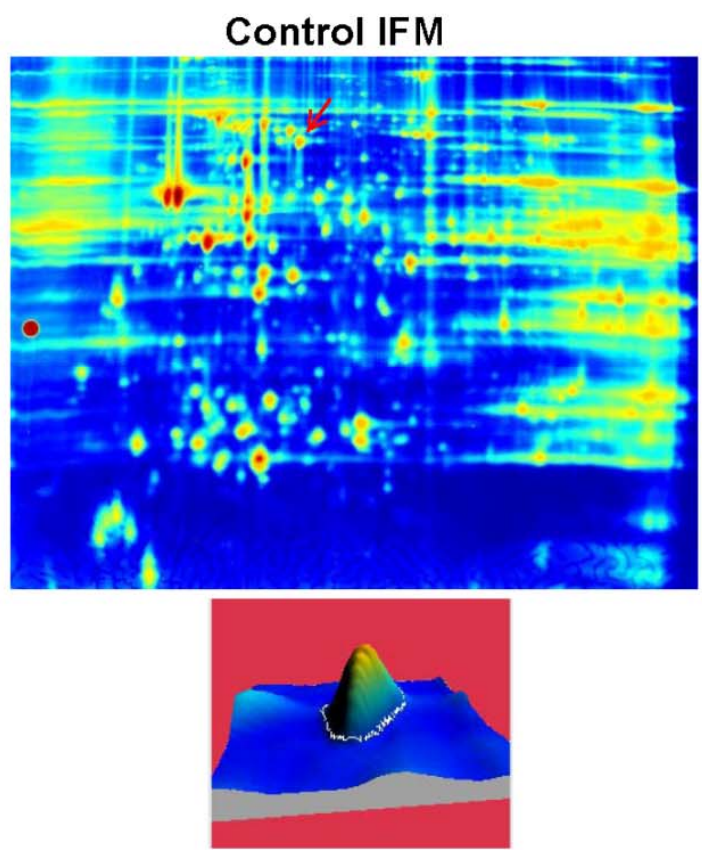

B
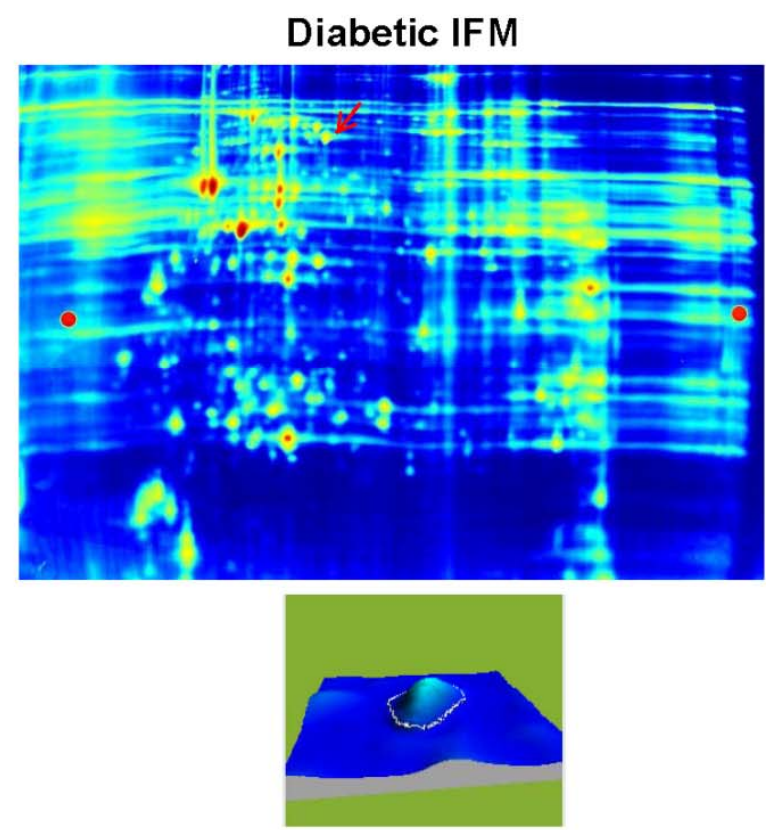
Figure 3.2

C
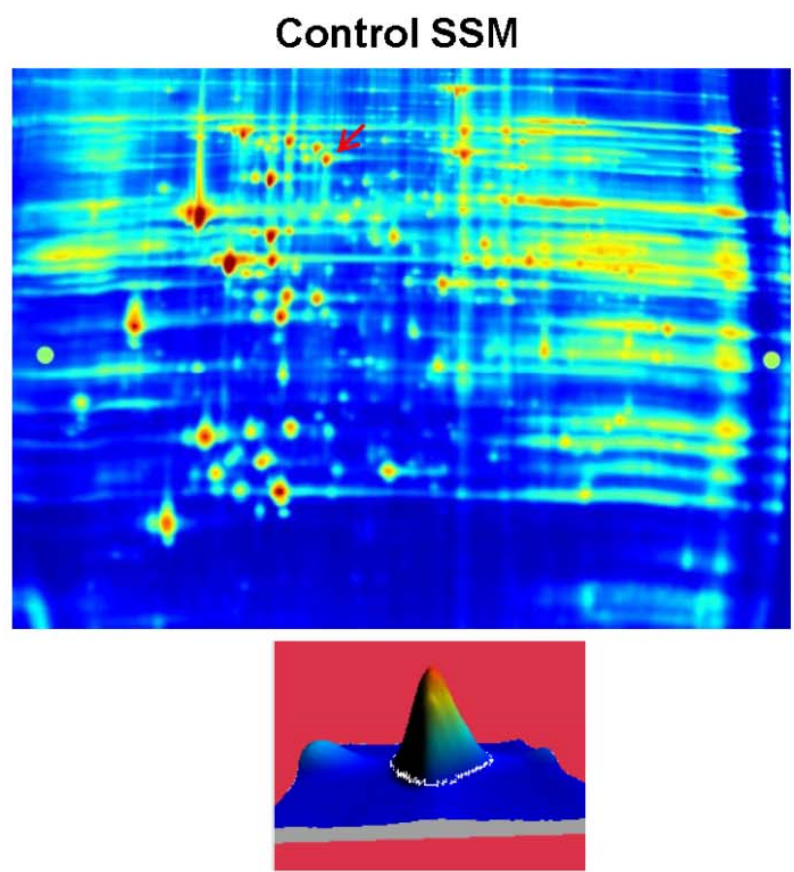

D

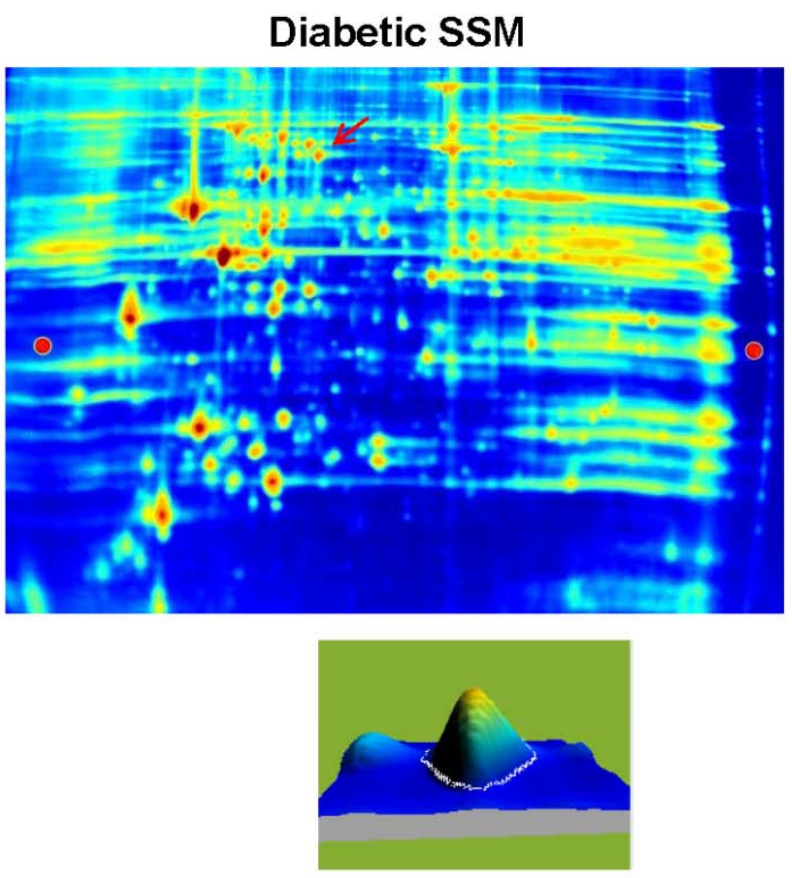


Figure 3.2. Representative 2D-DIGE gels. Samples were labeled with either Cy3 or Cy5 alternating between control and diabetic SSM and IFM. A control and treated were run on the same gel and an internal standard was labeled with Cy2 and run on every gel for gel to gel comparisons. (A) A representative gel showing Cy3 labeled control IFM and (B) is the same gel showing Cy5 labeled diabetic IFM. (C) A representative gel showing Cy3 labeled control SSM and (D) is the same gel showing Cy5 labeled control SSM. Below the gel images are individual spots that were identified as Grp75, (indicated by the red arrow) and quantitative differences were assessed by examination of peak density. 
Figure 3.3

A

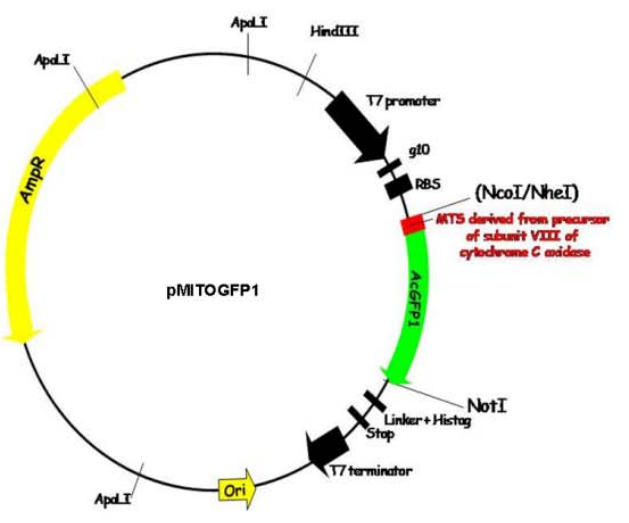

B

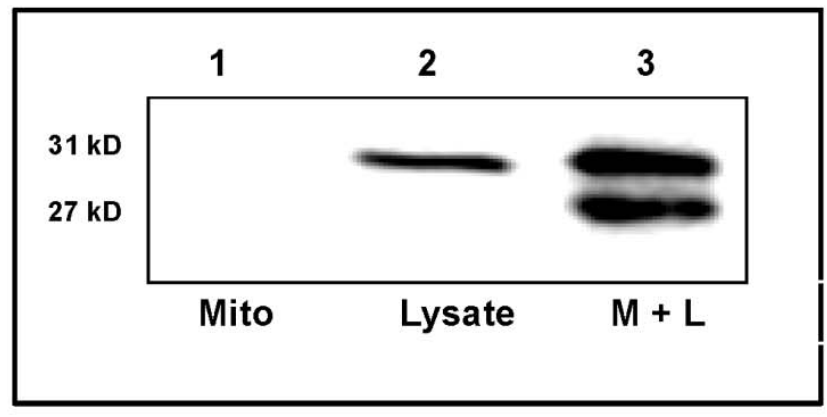


Figure 3.3. pMITOGFP1 construct. (A) pMITOGFP1 plasmid was generated and inserted into a bacterial cell free protein expression system to produce MitoGFP1 protein. The MITOGFP1 gene consists of an Nterminal mitochondrial targeting sequence derived from cytochrome $\mathrm{C}$ oxidase subunit VIII (red) fused with AcGFP1 (green). The MITOGFP1 gene was cloned into pIVEX2.3d at restriction sites Not I and blunted Nco I/Nhe I sites. (B) Western blot analysis of MitoGFP1 protein import into isolated cardiac mitochondria probed for AcGFP in samples containing $40 \mu \mathrm{g}$ of mitochondria only (lane 1), $0.05 \mu \mathrm{l}$ of MitoGFP1 protein lysate (lane

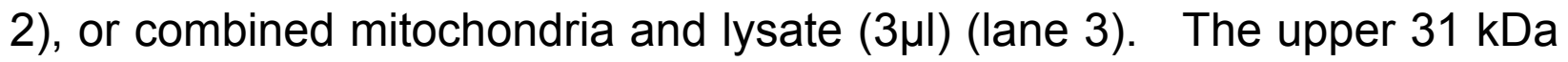
band represents the full length precursor MitoGFP1 in transit and yet to be processed while the lower $27 \mathrm{kDa}$ band represents a mature cleaved MitoGFP1 protein residing within the mitochondrial matrix. 
Figure 3.4

A

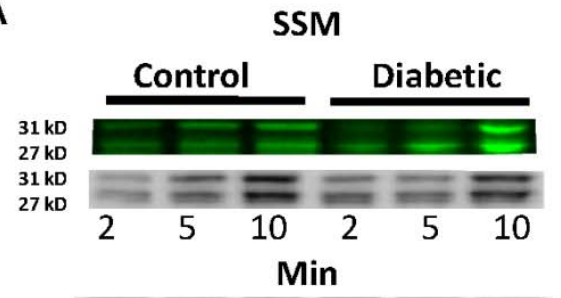

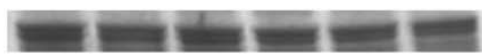

C

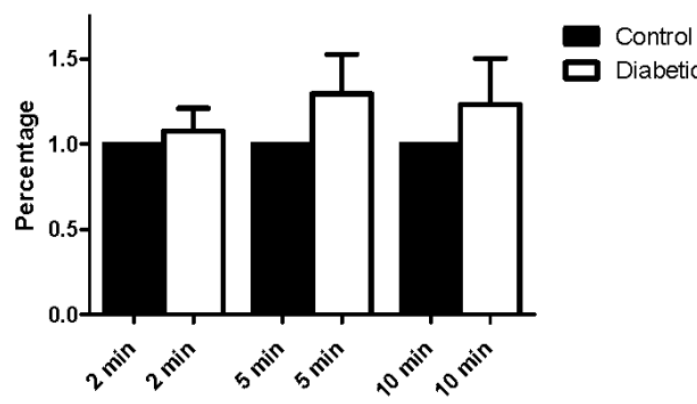

B

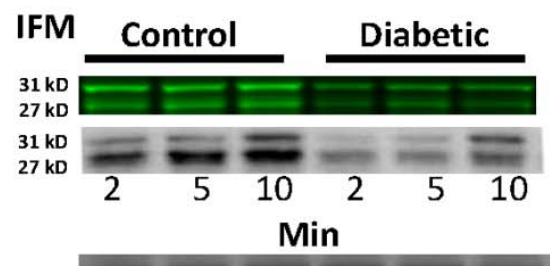

D

IFM

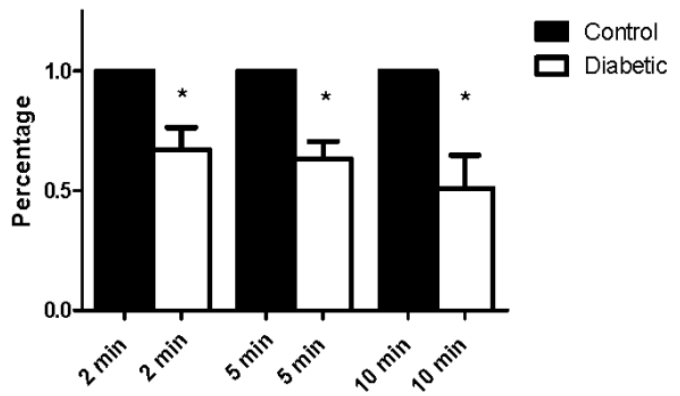


Figure 3.4. Mitochondrial protein import. Effect of type 1 diabetes mellitus on MitoGFP1 import in SSM and IFM subpopulations. Cardiac mitochondrial subpopulations from control and diabetic mice were isolated and incubated with $3 \mu \mathrm{l}$ of MitoGFP1 protein lysate at 2, 5, and 10 minute time points. Representative Western blots from (A) SSM and (B) IFM protein import assay. Graphical representation of mitochondrial protein import performed in (C) SSM and (D) IFM control and diabetic subpopulations. Diabetic values are based upon percent of control per corresponding time point. Control for protein loading was confirmed by Ponceau staining. Values are represented as mean \pm SEM; $\mathrm{N}=8$ per group. ${ }^{*} p<0.05$ for control vs. diabetic for all groups. Control for protein loading was confirmed with Ponceau staining. 
Figure 3.5

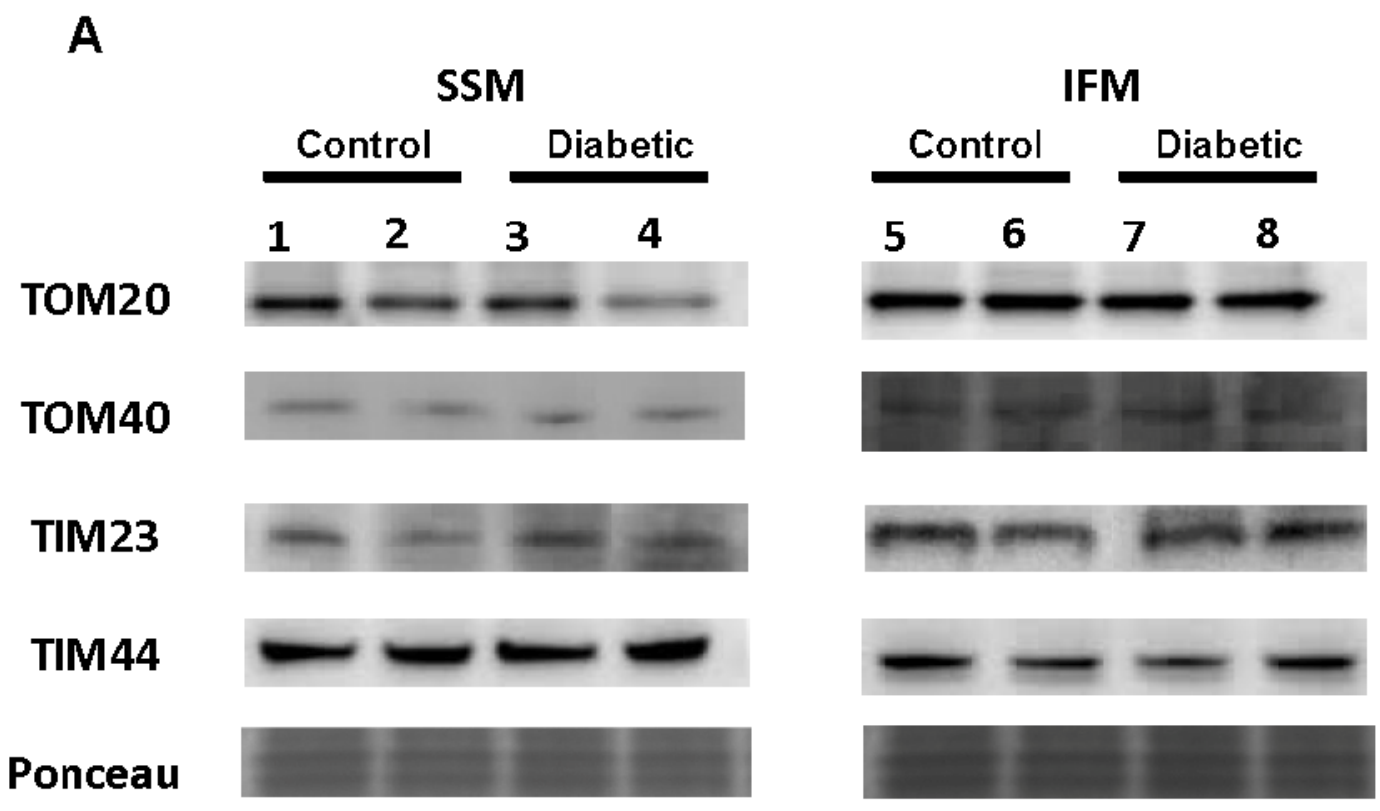


Figure 3.5

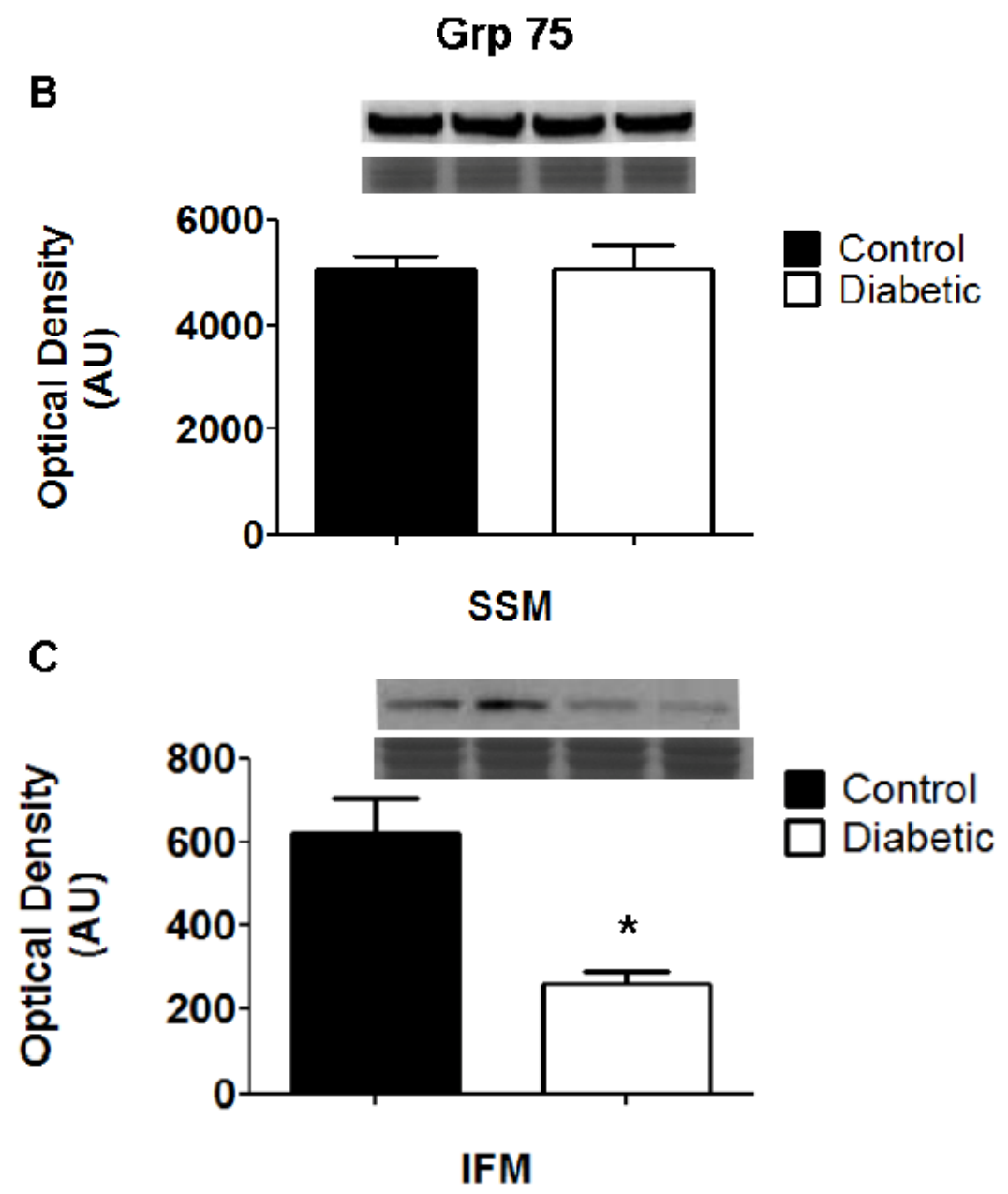


Figure 3.5. Western blot analysis of protein import constituents. Key proteins involved in mitochondrial protein import were assessed using Western Blot analyses. (A) Control SSM (lanes 1-2), Diabetic SSM (lanes 3-4), Control IFM (lanes 5-6), Diabetic IFM (lanes 7-8); translocases of the outer mitochondrial membrane TOM20, TOM40 and inner mitochondrial membrane TIM23, TIM44 protein expression. Representative Western blots and densitometry analysis for Grp75 protein expression in (A) SSM, and (B) IFM from control and diabetic hearts. Values are represented as mean \pm SEM; $N=5$ for each group. ${ }^{*} p<0.05$ for control vs. diabetic for all groups. Control for protein loading was confirmed with Ponceau staining. 


\section{Table 3.1}

\begin{tabular}{|c|c|c|}
\hline Protein Name & $\begin{array}{l}\text { SSM Contol/ SSM } \\
\text { Diabetic }\end{array}$ & $\begin{array}{l}\text { IFM Control/ } \\
\text { IFM Diabetic }\end{array}$ \\
\hline Mitochondrial Fatty Acids Beta-oxidation & & \\
\hline $\begin{array}{l}\text { Hydroxyacyl-coenzyme A dehydrogenase } 3 \\
\text { ketoacyl coenzyme A }\end{array}$ & 1.31 & 1.41 \\
\hline Mitochondrial trifunctional protein, beta subunit & 1.39 & 1.37 \\
\hline Acetyl-coenzyme A acyl transferase 2 & NS & 1.23 \\
\hline $\begin{array}{l}\text { Acyl coenzyme A dehydrogenase, medium } \\
\text { chain }\end{array}$ & NS & 1.14 \\
\hline $\begin{array}{l}\text { Acyl Coenzyme A dehydrogenase, very long } \\
\text { chain }\end{array}$ & 1.31 & 1.28 \\
\hline 2,4-dienoyl CoA reductase 1 & 1.30 & 1.40 \\
\hline Enoyl coenzyme hydratase 1 & NS & 1.26 \\
\hline Carnitine palmitoyltransferase 1 & .80 & 1.37 \\
\hline Mitochondrial Respiratory Chain & & \\
\hline $\begin{array}{l}\text { ATP synthase, } \mathrm{H}+\text { transporting, mitochondrial } \\
\text { F1 complex, Beta subunit }\end{array}$ & 1.31 & 1.20 \\
\hline $\begin{array}{l}\text { ATP synthase, } \mathrm{H}+\text { transporting, FO complex } \\
\text { subunit } \mathrm{F}\end{array}$ & .73 & 1.19 \\
\hline $\begin{array}{l}\text { ATP synthase, } \mathrm{H}+\text { transporting, mitochondrial } \\
\text { F1 complex, O subunit }\end{array}$ & NS & 1.13 \\
\hline
\end{tabular}




\begin{tabular}{|l|l|l|}
\hline $\begin{array}{l}\text { ATP synthase, H+ transporting, mitochondrial } \\
\text { FO, subunit b, isoform 1 }\end{array}$ & NS & 1.20 \\
\hline $\begin{array}{l}\text { NADH dehydrogenase ubiquinone flavoprotein } \\
1\end{array}$ & NS & 1.17 \\
\hline $\begin{array}{l}\text { NADH dehydrogenase ubiquinone 1 alpha } \\
\text { subcomplex 8 }\end{array}$ & .79 & NS \\
\hline $\begin{array}{l}\text { NADH dehydrogenase (ubiquinone) 1 alpha } \\
\text { subcomplex 12 }\end{array}$ & NS & 1.40 \\
\hline $\begin{array}{l}\text { Ubiquinol cytochrome c reductase, Rieske iron } \\
\text { sulfur unit polypeptide 1 }\end{array}$ & NS & .83 \\
\hline Cytochrome C-1 & .73 & NS \\
\hline Cytochrome Coxidase subunit IV isoform 1 & .85 & NS \\
\hline $\begin{array}{l}\text { Cytochrome C oxidase subunit VIb polypeptide } \\
1\end{array}$ & NS & 1.17 \\
\hline $\begin{array}{l}\text { Electron transfer flavoprotein-ubiquinone } \\
\text { oxidoreductase }\end{array}$ & NS & 1.43 \\
\hline Electron transfer flavoprotein subunit alpha & NS & .87 \\
\hline Electron transfer flavoprotein beta polypeptide & .83 & 1.34 \\
\hline TCA Cycle & NS \\
\hline Aconitase 2 & NS & \\
\hline
\end{tabular}




\begin{tabular}{|c|c|c|}
\hline Isocitrate dehydrogenase 2 (NADP+) & .86 & 1.33 \\
\hline Pyruvate dehydrogenase (lipoamide) beta & .70 & NS \\
\hline Creatine Kinase & .68 & NS \\
\hline Transport Proteins & & \\
\hline Adenine nucleotide translocator, member 4 & NS & 1.30 \\
\hline Vdac (Vdac-1) & NS & .87 \\
\hline $\begin{array}{l}\text { Solute carrier family 25, member } 3 \text { phosphate } \\
\text { carrier }\end{array}$ & NS & 1.47 \\
\hline Grp75 & NS & 1.20 \\
\hline Structural & & \\
\hline Inner membrane protein immt, mitofilin & NS & 1.36 \\
\hline Miscellaneous & & \\
\hline Myoglobin & NS & .66 \\
\hline Es 1 protein & NS & 1.25 \\
\hline Multimerin 2 & NS & 1.13 \\
\hline
\end{tabular}


Hydroxysteroid dehydrogenase like 2, isoform CRA_c

Mlrq-like protein

NS 
Table 3.1. ITRAQ Proteomic analysis of mitochondrial subpopulations from control and diabetic hearts. ITRAQ analysis of proteins identified and significantly changing, categorized into groups consisting of fatty acid oxidation (FAO), mitochondrial respiratory chain, citric acid cycle (TCA), transport proteins, structural and miscellaneous proteins in isolated SSM and IFM from control and diabetic hearts. Cells highlighted in red represent decreased protein expression in the diabetic mitochondria compared to control, while cells highlighted in green are increased in diabetic vs. control. All values presented indicate a significant difference of at least ${ }^{*} p<0.05$ for control vs. diabetic groups, while NS represents no significant differences between any groups. $\mathrm{N}=4$ for each group. 


\section{Table 3.2}

\begin{tabular}{|c|c|c|}
\hline Protein Name & $\begin{array}{l}\text { Difference SSM } \\
\text { Diabetic vs. SSM } \\
\text { Control }\end{array}$ & $\begin{array}{l}\text { Difference IFM } \\
\text { Diabetic vs. IFM } \\
\text { Control }\end{array}$ \\
\hline Mitochondrial Fatty Acids Beta-oxidation & & \\
\hline $\begin{array}{l}\text { Very long-chain specific Acyl-CoA } \\
\text { Dehydrogenase }\end{array}$ & -1.14 & -1.25 \\
\hline Long-Chain specific Acyl-CoA Dehydrogenase & NS & -1.21 \\
\hline $\begin{array}{l}\text { Isoform } 2 \text { of 2-oxoglutarate dehydrogenase E1 } \\
\text { Complex }\end{array}$ & NS & -1.27 \\
\hline Medium-chain specific acyl-coA dehydrogenase & -1.45 & -1.31 \\
\hline mitochondrial acyl-CoA thioesterase 1 & NS & -1.10 \\
\hline $\begin{array}{l}\text { Delta(3,5)-Delta(2,4)-Dienoyl-COA } \\
\text { ISOMERASE }\end{array}$ & 2.02 & -1.11 \\
\hline Mitochondrial Respiratory Chain & & \\
\hline $\begin{array}{l}\text { Isoform } 1 \text { of NADH Dehydrogenase } \\
\text { (Ubiquinone) Flavoprotein2 }\end{array}$ & 1.25 & -1.18 \\
\hline NADH dehydrogenase iron-sulfur protein 2 & NS & -1.15 \\
\hline
\end{tabular}




\begin{tabular}{|l|l|l|}
\hline $\begin{array}{l}\text { NADH-Ubiquinone Oxidoreductase } 75 \text { kda } \\
\text { subunit }\end{array}$ & -1.54 & -1.315 \\
\hline $\begin{array}{l}\text { NADH Dehydrogenase Ubiquinone 1 Alpha } \\
\text { subcomplex subunit 10 }\end{array}$ & NS & -1.23 \\
\hline $\begin{array}{l}\text { NADH Dehydrogenase Ubiquinone Iron-sulfur } \\
\text { Protein } 8\end{array}$ & NS & -1.15 \\
\hline $\begin{array}{l}\text { NADH dehydrogenase (ubiquinone) iron-sulfur } \\
\text { protein } 3\end{array}$ & NS & -1.15 \\
\hline $\begin{array}{l}\text { NADH dehydrogenase (ubiquinone) iron-sulfur } \\
\text { protein } 4\end{array}$ & NS \\
\hline $\begin{array}{l}\text { Electron Transfer Flavoprotein-ubiquinone } \\
\text { oxidoreductase }\end{array}$ & 2.83 & -1.10 \\
\hline Ubquinol cytochrome c redcutase core protein 1 & NS & -1.70 \\
\hline $\begin{array}{l}\text { Succinate dehydrogenase (ubiquinone) iron- } \\
\text { sulfur unit }\end{array}$ & NS \\
\hline Cytochrome b-cl complex c & NS & -1.16 \\
\hline
\end{tabular}




\begin{tabular}{|l|l|l|}
\hline Atp synthase subunit beta & NS & -1.25 \\
\hline Atp synthase subunit O & 1.13 & -1.67 \\
\hline ATP5a1 ATP synthase subunit alpha & NS & -1.21 \\
\hline TCA Cycle & 1.15 & \\
\hline Aldehyde dehydrogenase & NS & -1.14 \\
\hline $\begin{array}{l}\text { Pyruvate dehydrogenase E1 component subunit } \\
\text { Beta }\end{array}$ & NS & -1.29 \\
\hline $\begin{array}{l}\text { Dihydrolipoyllysine-residue acetyltrasnferase } \\
\text { component of pyruvate dehydrogenase complex }\end{array}$ & 1.50 & -1.34 \\
\hline Malate dehydrogenase & & -1.25 \\
\hline & 2.3 & \\
\hline & & \\
\hline
\end{tabular}




\begin{tabular}{|l|l|l|}
\hline Aspartate aminotransferase & NS & 1.96 \\
\hline ubiquitin protein ligase E3C & 1.34 & -2.3 \\
\hline Oxidative Stress Related & & \\
\hline Prdx3 & NS & \\
\hline Transport Proteins & NS & -1.23 \\
\hline Heat Shock Protein 60 & NS & \\
\hline IMMT Mitofilin & -1.40 & -1.326 \\
\hline Grp75 & NS & \\
\hline & & -1.36 \\
\hline
\end{tabular}




\section{Table 3.2. 2D-DIGE proteomic analysis of mitochondrial}

subpopulations from control and diabetic hearts. 2D-DIGE analysis of proteins identified and significantly changing, categorized into groups consisting of fatty acid oxidation (FAO), mitochondrial respiratory chain, citric acid cycle (TCA), amino acid metabolism, oxidative stress related, transport proteins, and structural proteins in isolated SSM and IFM from control and diabetic hearts. Cells highlighted in red represent decreased protein expression in the diabetic mitochondria compared to control, while cells highlighted in green are increased in diabetic vs. control. All values presented indicate a significant difference of at least ${ }^{*} p<0.05$ for control vs. diabetic groups, while NS represents no significant differences between any groups. $\mathrm{N}=4$ for each group. 


\section{Table 3.3}

\begin{tabular}{|c|c|c|}
\hline Protein Diabetic IFM & $\begin{array}{l}\text { Peptide Sequence } \\
\text { Diabetic IFM }\end{array}$ & $\begin{array}{l}\text { Post Translational } \\
\text { Modification } \\
\text { Diabetic IFM }\end{array}$ \\
\hline $\begin{array}{l}\text { ATP synthase, } \mathrm{H}+\text { transporting } \\
\text { mitochondrial } \mathrm{F} 1 \text { complex, beta } \\
\text { subunit }\end{array}$ & ALVYGQMNEPPGAR & Oxidation(M)@7; Cation:K(E)@9 \\
\hline $\begin{array}{l}\text { Ubiquinol cytochrome c reductase } \\
\text { core protein } 2\end{array}$ & NALANPLYCPDYR & $\begin{array}{l}\text { Carbamidomethyl(C)@9; } \\
\text { Dioxidation(Y)@12; Arg- } \\
\text { >GluSA(R)@13 }\end{array}$ \\
\hline $\begin{array}{c}\text { solute carrier family } 25 \\
\text { (mitochondrial carrier, adenine } \\
\text { nucleotide translocator), member } \\
4 \text { [Mus musculus] }\end{array}$ & GADIMYTGTLDCWR & $\begin{array}{l}\text { Carbamidomethyl(C)@12; } \\
\text { Dioxidation(W)@13; Arg- } \\
\text { >GluSA(R)@14 }\end{array}$ \\
\hline $\begin{array}{l}\text { ubiquinol-cytochrome c reductase } \\
\text { core protein } 1\end{array}$ & VYEEDAVPGLTPCR & $\begin{array}{c}\text { Oxidation(P)@8; } \\
\text { Oxidation(P)@12; } \\
\text { Carbamidomethyl(C)@13; Arg- } \\
\text { >GluSA(R)@14 }\end{array}$ \\
\hline $\begin{array}{l}\text { ubiquinol-cytochrome c reductase } \\
\text { core protein } 1\end{array}$ & NALVSHLDGTTPVCEDIGR & $\begin{array}{c}\text { Dioxidation(P)@12; } \\
\text { Carbamidomethyl(C)@14; Arg- } \\
\text { >GluSA(R)@19 }\end{array}$ \\
\hline $\begin{array}{c}\text { acetyl-Coenzyme A } \\
\text { acetyltransferase } 1\end{array}$ & $\begin{array}{c}\text { ENGTITAANASTLNDGAAALV } \\
\text { LMTAEAA } \\
\text { QR }\end{array}$ & $\begin{array}{l}\text { Oxidation(N)@2; } \\
\text { Deamidated(N)@9 }\end{array}$ \\
\hline Grp75 & MKETAENYLGHTAK & Oxidation(M)@1 \\
\hline $\begin{array}{l}\text { ATP synthase, } \mathrm{H}+\text { transporting, } \\
\text { mitochondrial F0 complex, } \\
\text { subunit b, isoform } 1\end{array}$ & QIQDAIDMEK & Oxidation(M)@8 \\
\hline Titin (Connectin) & KMEAPPPKAPKKR & Dioxidation(P)@5 \\
\hline $\begin{array}{c}\text { NADH dehydrogenase } \\
\text { (ubiquinone) } 1 \text { beta subcomplex } 8\end{array}$ & $\begin{array}{c}\text { VEDYEPYPDDGMGYGDYPML } \\
\text { PNR }\end{array}$ & Oxidation(D)@3 \\
\hline $\begin{array}{l}\text { inner membrane protein, } \\
\text { mitochondrial(Mitofilin) }\end{array}$ & RVAQDWLKEAR & $\begin{array}{l}\text { Deamidated(Q)@4; } \\
\text { Oxidation(W)@6 }\end{array}$ \\
\hline $\begin{array}{l}\text { dodecenoyl-Coenzyme A delta } \\
\text { isomerase }\end{array}$ & SLHMYLEK & Oxidation(M)@4 \\
\hline $\begin{array}{l}\text { ATP synthase, } \mathrm{H}+\text { transporting } \\
\text { mitochondrial F1 complex, beta } \\
\text { subunit }\end{array}$ & TREGNDLYHEMIESGVINLK & Deamidated(N)@5 \\
\hline $\begin{array}{l}\text { ATP synthase, } \mathrm{H}+\text { transporting } \\
\text { mitochondrial F1 complex, beta } \\
\text { subunit }\end{array}$ & $\begin{array}{c}\text { GSITSVQAIYVPADDLTDPAPA } \\
\text { TTFAHLDATTVLSR }\end{array}$ & $\begin{array}{c}\text { Acetyl@N-term; } \\
\text { Deamidated(Q)@7; } \\
\text { Carbamidomethyl(D)@14 }\end{array}$ \\
\hline
\end{tabular}




\begin{tabular}{|c|c|c|}
\hline $\begin{array}{l}\text { ATP synthase, } \mathrm{H}+\text { transporting, } \\
\text { mitochondrial F1 complex, alpha } \\
\text { subunit, isoform } 1\end{array}$ & VVDALGNAIDGK & Deamidated(N)@7 \\
\hline $\begin{array}{l}\text { 3-ketoacyl-CoA thiolase, } \\
\text { mitochondrial (Beta-ketothiolase) }\end{array}$ & $\begin{array}{c}\text { AALSAGKVPPETIDSVIVGNV } \\
\text { MQSSSDAAYLAR }\end{array}$ & Deamidated(N)@20 \\
\hline $\begin{array}{l}\text { mitochondrial trifunctional } \\
\text { protein, beta }\end{array}$ & DNGIRPSSLEQMAK & Deamidated(N)@2 \\
\hline $\begin{array}{l}\text { mitochondrial trifunctional } \\
\text { protein, beta }\end{array}$ & $\begin{array}{c}\text { LKPAFIKPYGTVTAANSSFLTD } \\
\text { GASAMLIMSEDR }\end{array}$ & Deamidated(N)@16 \\
\hline $\begin{array}{c}\text { glutamate oxaloacetate } \\
\text { transaminase 2, mitochondrial } \\
\text { [Mus musculus] }\end{array}$ & DDNGKPYVLPSVR & Deamidated(N)@3 \\
\hline $\begin{array}{c}\text { glutamate oxaloacetate } \\
\text { transaminase 2, mitochondrial } \\
\text { [Mus musculus] }\end{array}$ & $\begin{array}{c}\text { KMNLGVGAYRDDNGKPYVL } \\
\text { PSVR }\end{array}$ & Deamidated(N)@13 \\
\hline $\begin{array}{l}\text { succinate dehydrogenase Fp } \\
\text { subunit [Mus musculus] }\end{array}$ & NTVIATGGYGR & Deamidated(N)@1 \\
\hline $\begin{array}{c}\text { solute carrier family } 25 \\
\text { (mitochondrial carrier, adenine } \\
\text { nucleotide translocator) }\end{array}$ & $\begin{array}{c}\text { GDQALSFLKDFLAGGIAAAVS } \\
\mathrm{K}\end{array}$ & Protein Terminal Acetyl@N-term \\
\hline $\begin{array}{c}\text { solute carrier family } 25 \\
\text { (mitochondrial carrier, adenine } \\
\text { nucleotide translocator) }\end{array}$ & GDQALSFLK & Protein Terminal Acetyl@N-term \\
\hline $\begin{array}{l}\text { ubiquinol-cytochrome c reductase } \\
\text { core protein } 1\end{array}$ & $\begin{array}{c}\text { TATFAQALQSVPETQVSILDN } \\
\text { GLR }\end{array}$ & Deamidated(N)@21 \\
\hline $\begin{array}{l}\text { ubiquinol-cytochrome c reductase } \\
\text { core protein } 1\end{array}$ & EVESIGAHLNAYSTR & Deamidated(N)@10 \\
\hline cytochrome c oxidase, subunit Va & LNDFASAVR & Deamidated(N)@2 \\
\hline cytochrome c oxidase, subunit Va & RLNDFASAVR & $\begin{array}{l}\text { Deamidated(N)@3; } \\
\text { Dehydrated(D)@4 }\end{array}$ \\
\hline $\begin{array}{l}\text { acyl-Coenzyme A dehydrogenase, } \\
\text { long-chain [Mus musculus] }\end{array}$ & SPAHGISLFLVENGMK & Deamidated(N)@13 \\
\hline Grp75 & ASNGDAWVEAHGK & Deamidated(N)@3 \\
\hline Grp75 & STNGDTFLGGEDFDQALLR & Deamidated(N)@3 \\
\hline
\end{tabular}




\begin{tabular}{|c|c|c|}
\hline Fumarate hydratase 1 & YPIEHGIITNWDDMEK & Methyl(H)@5 \\
\hline Fumarate hydratase 1 & $\begin{array}{c}\text { LNDHFPLVVWQTGSGTQTNM } \\
\text { NVNEVISNR }\end{array}$ & Deamidated(Q)@17 \\
\hline $\begin{array}{l}\text { ATP synthase, } \mathrm{H}+\text { transporting, } \\
\text { mitochondrial F0 complex, } \\
\text { subunit F }\end{array}$ & $\begin{array}{c}\text { RQASGGPVDIGPEYQQDLDRE } \\
\text { LYK }\end{array}$ & $\begin{array}{l}\text { Deamidated(Q)@2; } \\
\text { Dehydrated(S)@4 }\end{array}$ \\
\hline $\begin{array}{c}\text { pyruvate dehydrogenase E1 alpha } \\
1 \text { [Mus musculus] }\end{array}$ & TREEIQEVRSKSDPIMLLK & Deamidated(R)@9 \\
\hline $\begin{array}{c}\text { superoxide dismutase 2, } \\
\text { mitochondrial [Mus musculus]; } \\
\text { Sod2 protein [Mus musculus] }\end{array}$ & FNGGGHINHTIFWTNLSPK & Deamidated(N)@2 \\
\hline $\begin{array}{l}\text { Carnitine O-acetyltransferase } \\
\text { (Carnitine acetylase) (CAT) }\end{array}$ & IWNSSLQSNKEPVGILTSNHR & Deamidated(N)@3 \\
\hline $\begin{array}{c}\text { NADH dehydrogenase } \\
\text { (ubiquinone) } 1 \text { beta subcomplex, } 9 \\
\text { [Mus musculus] }\end{array}$ & $\begin{array}{l}\text { EAEEEFWQNQHPQPYIFPDSP } \\
\text { GGTSFER }\end{array}$ & Deamidated(N)@9 \\
\hline $\begin{array}{l}\text { 3-hydroxyacyl CoA } \\
\text { dehydrogenase }\end{array}$ & $\begin{array}{l}\text { LDKFAAEHTIFASNTSSLQITNI } \\
\text { ANATTR }\end{array}$ & Deamidated(N)@14 \\
\hline $\begin{array}{l}\text { Aldehyde dehydrogenase family } \\
6 \text {, subfamily A1 }\end{array}$ & $\begin{array}{c}\text { NHGVVMPDANKENTLNQLV } \\
\text { GAAFGAAGQR }\end{array}$ & Deamidated(N)@10 \\
\hline $\begin{array}{c}\text { Aldehyde dehydrogenase family } \\
6 \text {, subfamily A1 }\end{array}$ & $\begin{array}{c}\text { IVNDNPYGNGTAIFTTNGATA } \\
\text { R }\end{array}$ & $\begin{array}{l}\text { Deamidated(N)@9; } \\
\text { Deamidated(N)@17 }\end{array}$ \\
\hline $\begin{array}{l}\text { dihydrolipoamide S- } \\
\text { succinyltransferase }\end{array}$ & NDVITVQTPAFAESVTEGDVR & Deamidated(N)@1 \\
\hline $\begin{array}{c}\text { NADH dehydrogenase } \\
\text { (ubiquinone) 1, subcomplex } \\
\text { unknown, } 2 \text { [Mus musculus] }\end{array}$ & MMNGRPGHEPLK & $\begin{array}{c}\text { Protein Terminal Acetyl@N-term; } \\
\text { Deamidated(N)@3 }\end{array}$ \\
\hline $\begin{array}{l}\text { isocitrate dehydrogenase } 3 \\
(\mathrm{NAD}+) \text {, gamma }\end{array}$ & $\begin{array}{l}\text { KAVLASMDNENMHTPDIGGQ } \\
\text { GTTSQAIQDII }\end{array}$ & Deamidated(N)@9 \\
\hline Protein Diabetic SSM & $\begin{array}{l}\text { Peptide Sequence } \\
\text { Diabetic SSM }\end{array}$ & $\begin{array}{c}\text { Post Translational Modification } \\
\text { Diabetic SSM }\end{array}$ \\
\hline $\begin{array}{l}\text { ATP synthase, } \mathrm{H}+\text { transporting } \\
\text { mitochondrial } \mathrm{F} 1 \text { complex, beta } \\
\text { subunit } \\
\end{array}$ & $\begin{array}{c}\text { IPSAVGYQPTLATDMGTMQE } \\
\mathrm{R}\end{array}$ & Oxidation(P)@2 \\
\hline $\begin{array}{l}\text { acetyl-Coenzyme A } \\
\text { acyltransferase } 2\end{array}$ & $\begin{array}{c}\text { VPPETIDSVIVGNVMQSSSDA } \\
\text { AYLAR }\end{array}$ & Oxidation(P)@2 \\
\hline
\end{tabular}




\begin{tabular}{|c|c|c|}
\hline $\begin{array}{l}\text { ubiquinol-cytochrome c reductase } \\
\text { core protein } 1\end{array}$ & VYEEDAVPGLTPCR & $\begin{array}{c}\text { Dioxidation(P)@12; } \\
\text { Carbamidomethyl(C)@13; Arg- } \\
\text { >GluSA(R)@14 }\end{array}$ \\
\hline $\begin{array}{l}\text { ubiquinol-cytochrome c reductase } \\
\text { core protein } 1\end{array}$ & $\begin{array}{c}\text { TATFAQALQSVPETQVSILDN } \\
\text { GLR }\end{array}$ & $\begin{array}{l}\text { Oxidation(F)@4; } \\
\text { Deamidated(Q)@6; } \\
\text { Deamidated(N)@21 }\end{array}$ \\
\hline $\begin{array}{c}\text { NADH dehydrogenase } \\
\text { (ubiquinone) } 1 \text { beta subcomplex } 8 \\
\text { [Mus musculus] }\end{array}$ & $\begin{array}{c}\text { VEDYEPYPDDGMGYGDYPML } \\
\text { PNR }\end{array}$ & Oxidation(D)@3 \\
\hline $\begin{array}{l}\text { ATP synthase, } \mathrm{H}+\text { transporting, } \\
\text { mitochondrial F1 complex, alpha } \\
\text { subunit, isoform } 1\end{array}$ & VVDALGNAIDGK & Deamidated(N)@7 \\
\hline $\begin{array}{l}\text { enoyl-Coenzyme A hydratase } \\
\text { (trifunctional protein), alpha } \\
\text { subunit }\end{array}$ & $\begin{array}{l}\text { SLNSEMDNILANLRLPAKPEV } \\
\text { SSDEDVQYR }\end{array}$ & Deamidated(N)@3 \\
\hline $\begin{array}{c}\text { glutamate oxaloacetate } \\
\text { transaminase 2, mitochondrial } \\
\text { [Mus musculus]; }\end{array}$ & ILIRPLYSNPPLNGAR & Deamidated(N)@13 \\
\hline $\begin{array}{c}\text { glutamate oxaloacetate } \\
\text { transaminase 2, mitochondrial } \\
\text { [Mus musculus]; }\end{array}$ & $\begin{array}{c}\text { MNLGVGAYRDDNGKPYVLPS } \\
\text { VR }\end{array}$ & Deamidated(N)@12 \\
\hline $\begin{array}{l}\text { ATP synthase, } \mathrm{H}+\text { transporting, } \\
\text { mitochondrial F0 complex, } \\
\text { subunit d }\end{array}$ & $\begin{array}{l}\text { NIIPFDQMTIDDLNEIFPETKLD } \\
\text { KK }\end{array}$ & Deamidated(N)@1 \\
\hline $\begin{array}{l}\text { Electron transfer flavoprotein- } \\
\text { ubiquinone oxidoreductase }\end{array}$ & IPVPILPGLPMNNHGNYIVR & Deamidated(N)@12 \\
\hline $\begin{array}{c}\text { oxoglutarate dehydrogenase } \\
\text { (lipoamide) }\end{array}$ & $\begin{array}{l}\text { SSLATMAHAQSLVEAQPNVD } \\
\text { KLVEDHLAVQSLIR }\end{array}$ & Deamidated(Q)@10 \\
\hline $\begin{array}{l}\text { ubiquinol cytochrome c reductase } \\
\text { core protein } 2 \text { [Mus musculus] }\end{array}$ & LPNGLVIASLENYAPLSR & Deamidated(N)@3 \\
\hline $\begin{array}{l}\text { ubiquinol cytochrome c reductase } \\
\text { core protein } 2 \text { [Mus musculus] }\end{array}$ & RGNNTTSLLSQSVAK & Deamidated(N)@3 \\
\hline $\begin{array}{l}\text { ubiquinol cytochrome c reductase } \\
\text { core protein } 2 \text { [Mus musculus] }\end{array}$ & $\begin{array}{c}\text { TSAAPGGVPLQPQDLEFTKLP } \\
\text { NGLVIASLENYAPLSR }\end{array}$ & $\begin{array}{l}\text { Deamidated(Q)@13; } \\
\text { Deamidated(N)@22 }\end{array}$ \\
\hline fumarate hydratase 1 & $\begin{array}{c}\text { LNDHFPLVVWQTGSGTQTNM } \\
\text { NVNEVISNR }\end{array}$ & Deamidated(Q)@17 \\
\hline Cytochrome c-1 & AANNGALPPDLSYIVR & Deamidated(N)@4 \\
\hline carnitine palmitoyltransferase 2 & $\begin{array}{l}\text { LSAVSGPAEYLQHSIVPTMHY } \\
\text { QDSLPR }\end{array}$ & Deamidated(Q)@12 \\
\hline $\begin{array}{c}\text { NADH dehydrogenase } \\
\text { (ubiquinone) } 1 \text { alpha subcomplex, } \\
8\end{array}$ & TDRPLPENPYHSR & $\begin{array}{l}\text { Dehydrated(S)@12; } \\
\text { Deamidated(R)@13 }\end{array}$ \\
\hline
\end{tabular}




\begin{tabular}{|c|c|c|}
\hline $\begin{array}{c}\text { pyruvate dehydrogenase E1 alpha } \\
1\end{array}$ & $\begin{array}{c}\text { KEIEDAAQFATADPEPPLEELG } \\
\text { YHIYSSDPPFEVR }\end{array}$ & Deamidated(Q)@8 \\
\hline $\begin{array}{c}\text { pyruvate dehydrogenase E1 alpha } \\
1\end{array}$ & MVNSNLASVEELKEIDVEVR & Deamidated(N)@3 \\
\hline $\begin{array}{c}\text { pyruvate dehydrogenase E1 alpha } \\
1\end{array}$ & TREEIQEVRSKSDPIMLLK & Deamidated(R)@9 \\
\hline
\end{tabular}


Table 3.3 Post-translational modifications (PTM) in mitochondrial subpopulations from control and diabetic hearts. Multidimensional protein identification technology (MudPIT) was used to identify posttranslational modifications (PTM) of proteins from SSM and IFM from control and diabetic mitochondria. Peptides presented represent only those peptides that have PTMs in the diabetic SSM (A) and IFM (B) and not present in the control group. 


\section{Chapter 4:}

\section{Mitochondrial Dysfunction in the Type 2 Diabetic Heart is Associated with Alterations in Spatially-Distinct Mitochondrial Proteomes}

As published in Am J Physiol Heart Circ Physiol.2010. June 11.

Erinne R. Dabkowski ${ }^{1,2,4}$, Walter A. Baseler ${ }^{1,2,4}$, Courtney L. Williamson ${ }^{1,2,4}$, Matthew Powell $^{5}$, Trust T. Razunguzwa ${ }^{5}$, Jefferson C. Frisbee ${ }^{1,3,4}$, and John M. Hollander ${ }^{1,2,4}$

\footnotetext{
${ }^{1}$ West Virginia University School of Medicine, ${ }^{2}$ Division of Exercise Physiology, ${ }^{3}$ Department of Physiology, ${ }^{4}$ Center for Cardiovascular and Respiratory Sciences, ${ }^{5}$ Protea Biosciences, Morgantown, WV 26506
}

Running Title: Mitochondrial Subpopulations and the Type 2 Diabetic Heart 


\begin{abstract}
Cardiac complications and heart failure are the leading cause of death in type 2 diabetic patients. Mitochondrial dysfunction is central in the pathogenesis of the type 2 diabetic heart. However, it is unclear whether this dysfunction is specific for a particular subcellular region. The purpose of this study was to determine whether mitochondrial dysfunction in the type 2 diabetic heart is specific to a spatially distinct subset of mitochondria. We investigated mitochondrial morphology, function, and proteomic composition of subsarcolemmal mitochondria (SSM) and interfibrillar mitochondria (IFM) in 18 week old $\mathrm{db} / \mathrm{db}$ mice. Oxidative damage was assessed in subpopulations through measuring lipid peroxidation by-products and nitrotyrosine residues. Proteomic profiles and post-translational modifications (PTM) were assessed in mitochondrial subpopulations utilizing iTRAQ ${ }^{\mathrm{TM}}$ and MudPIT technologies, respectively. SSM from $\mathrm{db} / \mathrm{db}$ hearts had altered morphology including a decrease in size and internal complexity, while $\mathrm{db} / \mathrm{db}$ IFM were increased in internal complexity. Db/db SSM displayed decreased state 3 respiration rates, electron transport chain (ETC) activities, ATP synthase activities, mitochondrial membrane potential $(\Delta \Psi \mathrm{m})$, and increased oxidative damage, with no change in IFM. Proteomic assessment revealed a greater impact on db/db SSM as compared to db/db IFM. Inner mitochondrial membrane (IMM) proteins including ETC, ATP synthesis, and mitochondrial protein import machinery were predominantly decreased. We provide evidence that mitochondrial dysfunction in the type 2 diabetic heart is associated with a specific subcellular locale. Further, mitochondrial morphological and functional indices are impacted differently during type 2 diabetic insult, and may result from modulation of spatially distinct mitochondrial proteomes.
\end{abstract}




\section{INTRODUCTION}

Type 2 diabetes mellitus is linked to reduced life span in humans due in part to cardiovascular complications and heart failure (19). Mitochondrial dysfunction and altered myocardial metabolism contribute to cardiovascular complications during type 2 diabetes mellitus $(3,4)$. Previous studies have observed a decrease in cardiac function, and decreased cardiac efficiency in $\mathrm{db} / \mathrm{db}$ mice, a model of type 2 diabetes mellitus (42). An increase in circulating free fatty acids present with type 2 diabetes mellitus leads to a pooling of fatty acids in the mitochondrion, facilitating an enhanced oxidative milieu. Examination of total mitochondria from $\mathrm{db} / \mathrm{db}$ mouse hearts revealed respiration and oxidative phosphorylation deficits, due in part to an increased oxidative environment in the mitochondrion (3).

The cardiomyocyte consists of two biochemically and spatially distinct mitochondrial subpopulations, the subsarcolemmal mitochondria (SSM) located beneath the plasma membrane and the interfibrillar mitochondria (IFM), situated between the myofibrils (33). These two mitochondrial subpopulations respond differently to physiological stimuli, including type 1 diabetes mellitus $(25,27,30,37)$. Previously, we reported differential effects on spatially distinct mitochondrial subpopulations in terms of morphology, function, and oxidative parameters following STZ-induced type 1 diabetic insult, with the IFM subpopulation being most affected (12). However, a previous study observed decreased complex II activity and mitochondrial DNA copy number in the SSM from the skeletal muscle of type 2 diabetic patients, with no significant effects on the IFM (37). Examination of mitochondrial cardiac proteomic profiles has revealed changes in specific mitochondrial constituents suggesting that the alteration of key proteins involved in substrate utilization, electron transport chain (ETC) function, antioxidant status, and other important mitochondrial processes may be associated with the pathogenesis of diabetes mellitus $(6,20,47)$. A recent study examining the impact of endurance exercise revealed distinct subpopulation-specific mitochondrial proteome alterations (23). Nevertheless, to date, no study has examined cardiac mitochondrial subpopulation response in a type 2 diabetic model.

The goal of the present study was to determine how spatially distinct mitochondrial subpopulations in the heart of $\mathrm{db} / \mathrm{db}$ mice are impacted, and to discern 
the effects on subpopulation-specific mitochondrial proteomes. Our results suggest that the SSM subpopulation displays greater dysfunction in the $\mathrm{db} / \mathrm{db}$ heart, which may be due to specific alterations in the SSM proteome. This data highlights the importance and relevance of taking into account subcellular location when examining mitochondria during diabetic insult. 


\section{MATERIALS AND METHODS}

\section{Experimental Animals}

The animal experiments in this study conformed to National Institutes of Health (NIH) Guidelines for the Care and Use of Laboratory Animals, and were approved by the West Virginia University Animal Care and Use Committee (WVU-ACUC). Male db/db mice, strain BKS.Cg-m +/+ Lepr ${ }^{d b} / J$, and wild-type (WT) littermate controls (Jackson Laboratories, Bar Harbor, ME) were housed in the West Virginia University Health Sciences Center animal facility. Mice were given unlimited access to a rodent diet and water. All mouse groups were aged to 18 weeks and then sacrificed for the following experiments.

\section{Preparation of Individual Mitochondrial Subpopulations}

At 18 weeks of age, $\mathrm{db} / \mathrm{db}$ mice and their littermate controls were sacrificed, and the hearts were excised. Hearts were rinsed in phosphate buffered saline (PBS, pH 7.4), then blotted dry and weighed. SSM and IFM were isolated on ice following the methods of Palmer et al. (33) with minor modifications $(12,13)$. Briefly, the ventricles were minced and homogenized 1:10 (w/v) in cold Chappel-Perry buffer (in mmol/L: 100 $\left.\mathrm{KCl}, 50 \mathrm{MOPS}, 5 \mathrm{MgSO}_{4} \cdot 7 \mathrm{H}_{2} \mathrm{O}, 1 \mathrm{EGTA}, 1 \mathrm{ATP} ; \mathrm{pH} 7.4\right)$ at $4^{\circ} \mathrm{C}$. The homogenates were than centrifuged at $700 \times g$ for 10 minutes. The supernatant containing the SSM was extracted and centrifuged again at $10,000 \times g$ to isolate SSM. The SSM pellet was washed and centrifuged two more times at 10,000 $\times g$ and then once more at 10,000 $\mathrm{xg}$ to obtain a clean SSM fraction. The remaining pellet from the $700 \times g$ spin was resuspended in KME buffer (in mmol/L: $100 \mathrm{KCl}, 50 \mathrm{MOPS}$, and 0.5 EGTA; pH 7.4) and exposed to $5 \mathrm{mg} / \mathrm{g}$ of trypsin for 10 minutes. After 10 minutes, the IFM pellet was diluted two-fold with buffer plus protease inhibitor cocktail (Biovision, Mountain View CA) to inhibit trypsin, and spun down at $700 \times g$ for 10 minutes. The IFM containing supernatant was saved and the pellet resuspended and spun down again at $700 \times g$ for 10 minutes to maximize IFM yield. Next, supernatants were combined and spun down at $10,000 \times g$ to yield the IFM. The IFM were washed several times and spun down at a final spin of $10,000 \times g$ for 10 minutes. Pellets were resuspended in a sucrose buffer containing (in mmol/L: 220 sucrose, 70 mannitol, $10 \mathrm{Tris} \cdot \mathrm{HCl}$, and $1 \mathrm{EDTA} ; \mathrm{pH} 7.4$ ), and 
protein concentrations were determined using the Bradford method with bovine serum albumin (BSA) as a standard (5).

\section{Mitochondria Size and Internal Complexity}

To index mitochondrial subpopulation size and complexity, we performed flow cytometry analyses using a FACS Calibur equipped with a 15 MW 488-nm argon laser and 633 red diode laser (Becton Dickinson, San Jose, CA) as previously described (12, 13). Briefly, Mitotracker deep red 633 (Invitrogen, Carlsbad, CA), which moves into intact mitochondria due to membrane potential, was used to selectively stain intact mitochondria (emission wavelength: $633 \mathrm{~nm}$, fluorescent 633 red diode laser) and debris excluded by gating for only mitotracker deep red 633 positive events (intact mitochondria). Forward scatter (FSC) and side scatter (SSC) detectors were used to examine size (FSC) and complexity (SSC) in isolated mitochondrial subpopulations and represented as histograms plotted against the number of gated events as previously described $(12,13)$. To confirm the absolute mitochondria size, we used a flow cytometry size calibration kit (Invitrogen), which uses a set of microsphere suspensions $(0.5-6 \mu \mathrm{m})$ to serve as reliable size references for flow cytometric analyses as previously described $(12,13)$. All flow cytometric measurements were performed under the supervision of the West Virginia University Flow Cytometry Core Facility.

\section{Electron Transport Chain (ETC) Respiration}

State 3 and state 4 respiration rates were assessed in isolated mitochondrial subpopulations as previously described $(7,8,21)$ with slight modifications (13). After the mitochondrial subpopulation isolation, samples were resuspended in respiration buffer containing (in mmol/L: $80 \mathrm{KCl}, 50 \mathrm{MOPS}, 1 \mathrm{EGTA}, 5 \mathrm{KH} 2 \mathrm{PO} 4$, and $1 \mathrm{mg} / \mathrm{ml} \mathrm{BSA}$ ) and equal volumes were loaded into a Gilson chamber (Gilson, Middleton, WI) attached to a Yellow Springs Instruments 5300 biological oxygen monitor (YSI, Yellow Springs, OH). The substrates glutamate $(5 \mathrm{mM})+$ malate $(5 \mathrm{mM})$, palmitoylcarnitine $(40 \mu \mathrm{M})$ was used to initiate maximal respiration and measurement of state $3(1 \mathrm{mM} \mathrm{ADP})$ and state 4 (ADP-limited) respiration were made as previously described (7). 


\section{Electron Transport Chain (ETC) Complex Activities}

ETC activities of complexes I, III and IV were measured spectrophotometrically as previously described $(12,46)$. Briefly, complex I activity was determined by measuring the oxidation of NADH at $340 \mathrm{~nm}$ as previously described (46). The assay mixture for complex I contained $25 \mathrm{mM}$ potassium phosphate buffer (pH 7.2), $5 \mathrm{mM}$ $\mathrm{MgCl}_{2}, 2 \mathrm{mM} \mathrm{KCN}, 2.5 \mathrm{mg} / \mathrm{ml}$ bovine serum albumin, $50 \mu \mathrm{M} N A D H, 10 \mu \mathrm{M}$ decylubiquinone and $2 \mu \mathrm{g} / \mathrm{ml}$ antimycin $A$. The reaction was initiated by adding purified mitochondria $(45 \mu \mathrm{g})$, and the enzyme activity was measured for 3 minutes, with values recorded every 10 seconds after the initiation of the reaction. The complex I specific activity was inhibited by $2 \mu \mathrm{g} / \mathrm{ml}$ of rotenone. Complex III activity was determined as previously described $(12,46)$ by following the reduction of cytochrome $c$ at $550 \mathrm{~nm}$ in the presence of reduced decylubiquinone $(50 \mu \mathrm{M})$. Briefly, the assay buffer for complex III consisted of $500 \mathrm{mM}$ sucrose, $2 \mathrm{mM}$ EDTA, $100 \mathrm{mM}$ Tris-HCl, (pH 7.4), $1 \mathrm{mM}$ cytochrome c, $200 \mathrm{mM} \mathrm{KCN}, 1 \mathrm{mg} / \mathrm{ml}$ Antimycin A and reduced decylubiquinone. Finally, complex IV activity was determined by measuring the oxidation of cytochrome $c$ at $550 \mathrm{~nm}$. Briefly, the assay mixture for complex IV consisted of $10 \mathrm{mM}$ phosphate buffer $(\mathrm{pH} \mathrm{7.4)}$ and $20 \mu \mathrm{M}$ reduced cytochrome c. Protein content was determined as described above (5), and values expressed as activities in nanomoles substrate consumed per minute per milligram of protein.

\section{ATP Synthase Activity}

ATP synthase activity was measured in frozen-thawed mitochondria as oligomycin-sensitive ATPase activity using an assay coupled with pyruvate kinase which converts the ADP to ATP and produces pyruvate from phosphoenolpyruvate. (16, 34, 39). Briefly, isolated mitochondria subpopulations were incubated in a buffer containing (in mmol/L: $20 \mathrm{HEPES}, 5 \mathrm{MgCl}_{2}, 100 \mathrm{KCl}, 5 \mathrm{KCN}, 2.5$ phosphoenolpyruvate, $0.2 \mathrm{NADH}$, and $0.1 \mathrm{mg} / \mathrm{ml}$ pyruvate kinase, $0.1 \mathrm{mg} / \mathrm{ml}$ lactate dehydrogenase; $\mathrm{pH} 7.5$ 8.0). The reaction was initiated by addition of ATP to a desired final concentration (1 $\mathrm{mM}$ ) and followed by the decrease in NADH absorption at $340 \mathrm{~nm}$ wavelength (16). Absorbance was measured on a Biotek Synergy HT plate reader (Biotek, Winooski, VT), and protein content was assessed as above (5) with final values expressed as 
micromoles consumed per minute per milligram of protein, which was equal to the micromoles of NADH oxidized per minute per milligram of protein.

\section{Mitochondrial Membrane Potential ( $\left.\Delta \Psi_{m}\right)$}

Mitochondrial membrane potential $\left(\Delta \Psi_{\mathrm{m}}\right)$ was measured by flow cytometry using the ratiometric dye 5,5',6,6'-tetrachloro-1,1',3,3'-tetraethylbenzimidazol carbocyanine iodide (JC-1) (Molecular Probes, Carlsbad, CA), which is a lipophilic cation that enters selectively into mitochondria (36). Isolated mitochondrial subpopulations were incubated with $\mathrm{JC}-1$ for 15 minutes at $37^{\circ} \mathrm{C}$, and 20,000 gated events were analyzed per sample. Changes in $\Delta \Psi \mathrm{m}$ are reflected in the degree of color change from green to orange as membrane potential increases. The shift to orange is due to the dye forming aggregates upon membrane polarization causing shifts in emitted light from $530 \mathrm{~nm}$ (green) to $590 \mathrm{~nm}$ (orange). Addition of $200 \mu \mathrm{M}$ of dinitrophenol (DNP), which collapses the $\Delta \Psi_{\mathrm{m}}$, was used as an assay control. Measurements were performed on freshly isolated mitochondria subpopulations.

\section{Western Blot Analyses}

SDS polyacrylamide gel electrophoresis (SDS-PAGE) was run on $4-12 \%$ gradient gels as previously described (26) with equal amounts of protein loaded for each study treatment. Relative amounts of subpopulation-specific mitochondrial adenine nucleotide translocase (ANT), cytochrome oxidase (COX IV), and ubiquinol cytochrome c reductase core protein 2 (UQCRC2) were assessed through the use of specific antibodies including: anti-ANT goat antibody (Product\# sc-9300; Santa Cruz Biotech, Santa Cruz, CA), anti-COX IV rabbit antibody (Product\# 4866; Cell Signaling, Danvers, MA), uncoupling protein 3 (UCP3; Product\# PA1-028; Affinity Bioreagents, Golden, $\mathrm{CO}$ ), and anti-Uqcrc2 mouse antibody (Product\# MS304; Mitosciences, Eugene, OR). The secondary antibody was a goat anti-rabbit IgG HRP conjugate (Product\# 10004301; Cayman Chemical, Ann Arbor, MI) for COXIV, donkey anti-goat IgG HRP conjugate (Product \#sc-2020; Santa Cruz Biotech, Santa Cruz, CA) for ANT and goatanti mouse conjugate (Produc\# 31430; Pierce Biotech, Rockford, IL) for Uqcrc2. Detection of signal was performed according to the manufacturer's instructions, using 
Pierce ECL Western Blotting Substrate (Pierce, Rockford, IL). Autoradiographic signals were assessed using a G:Box Bioimaging System (Syngene, Frederick, MD), and the data captured and analyzed using GeneSnap/GeneTools software (Syngene, Frederick, $M D)$. Control for protein loading was confirmed by Ponceau staining.

\section{Lipid Peroxidation Products}

Peroxidation of lipids was assessed by measurement of malondialdehyde (MDA) and 4-hydroxyalkenal (4-HAE), stable end products formed from the oxidation of polyunsaturated fatty acids and esters, as previously described $(12,13)$. Equal volumes of freshly isolated mitochondrial subpopulations were analyzed for MDA and 4-HAE using a colorimetric assay kit (Oxford Biomedical Research, Oxford, MI). This assay is based on the reaction of a chromogenic reagent, $N$-methyl-2-phenylindole, with MDA and $4-\mathrm{HAE}$ at $45^{\circ} \mathrm{C}$. One molecule of either MDA or 4-HAE reacts with two molecules of the reagent to yield a stable chromophore with a maximal absorbance at $586 \mathrm{~nm}$. Absorbance was measured on a Biotek Synergy HT plate reader (Biotek, Winooski, VT), and protein content was assessed as described above (5) with final values expressed per milligram of protein.

\section{Protein Nitrotyrosine Content}

Oxidatively-modified proteins were examined by measuring nitrosylated groups introduced into protein side chains using a commercially available kit (Cell Sciences, Canton, MA) as previously described (12). Nitrotyrosine-containing proteins were measured using a solid-phase enzyme-linked immunosorbent assay based on the sandwich principle. Samples were incubated in microtiter wells coated with antibodies recognizing nitrotyrosine residues. After an incubation period and a wash, a biotinylated secondary antibody (tracer) was added, followed by a wash and the addition of a streptavidin-peroxidase conjugate. Color development was measured spectrophotometrically at $450 \mathrm{~nm}$ after the addition of tetramethylbenzidine using a Biotek Synergy HT plate reader (Biotek, Winooski, VT), and values were compared against known nitrotyrosine standards. 


\section{iTRAQ ${ }^{\mathrm{TM}}$ Labeling}

Pooled SSM and IFM subpopulations $(n=4)$ from $\mathrm{db} / \mathrm{db}$ and WT hearts were lysed and precipitated overnight in acetone at $-20^{\circ} \mathrm{C}$ and pellets were resuspended in $20 \mu \mathrm{L}$ of $0.5 \mathrm{M}$ triethylammonium bicarbonate (TEAB; $\mathrm{pH}$ 8.5). Protein contents were determined using a 2-D Quant Kit (Amersham, Piscataway, NJ) and $100 \mu \mathrm{g}$ of each pooled sample was than denatured with $0.1 \%$ SDS and reduced with $5 \mathrm{mM}$ tris-(2carboxyethyl) phosphine (TCEP). After incubation for 1 hour at $60^{\circ} \mathrm{C}$, cysteines were blocked with $10 \mathrm{mM}$ methyl methane thiosulfonate (MMTS) in isopropanol, and the samples were incubated at room temperature for 10 minutes. Addition of $10 \mu \mathrm{L}$ of sequencing grade trypsin (Applied Biosystems, Foster City, CA) was added in a trypsin/protein ratio of $1: 20$, and the samples incubated at $37^{\circ} \mathrm{C}$ overnight. Digested samples were labeled with the ITRAQ ${ }^{\mathrm{TM}}$ reagents following the protocol provided by the vendor (Applied Biosystems, Foster City, CA).

After digestion and $\mathrm{iTRAQ}{ }^{\mathrm{TM}}$ labeling, the ultra-complex protein digests were combined to create a $400 \mu \mathrm{g}$ pooled protein digest sample that contained equal fractions of each of the four labeled samples for subsequent Multi Dimensional Protein Identification Technology (MudPIT) analysis (28). After lyophilization, the digest mixture was reconstituted in strong cation exchange (SCX) loading buffer ( $5 \mathrm{mM}$ ammonium formate in $20 \%$ acetonitrile; $\mathrm{pH} 3.0$ ) to be fractionated with SCX SpinTips (Protea Biosciences, Morgantown, WV) per the manufacturer's protocol. Briefly, the sample solution was loaded centrifugally onto the SCX SpinTip. The non-adsorbing solution that passed through the SCX SpinTip was collected. Eight different elution solutions were used to fractionate the peptides $(20,60,100,150,200,250,400$, and $500 \mathrm{mM}$ ammonium formate in $10 \%$ acetonitrile) in a step-wise manner, for a total of nine sample fractions. The collected fractions were cleaned by repeated lyophilization and reconstituted in a $0.1 \mathrm{M}$ acetic acid solution, and then lyophilized to dryness. The fractions were then submitted for LC-MALDI TOF/TOF mass spectral analysis for protein identification, characterization, and differential expression analysis. 


\section{Mass Spectrometry (MS) Analyses with iTRAQ ${ }^{\mathrm{TM}}$ Labeling}

The LC-MALDI mass spectrometry system utilized was an ABI Tempo LC MALDI spotter with Tempo LC MALDI v.2.00.09 data acquisition and processing software. Lyophilized SCX sample fractions were reconstituted in LC aqueous run buffer $(0.1 \%$ trifluoroacetic acid, $2 \%$ acetonitrile) and was injected onto a Zorbax $\mathrm{C}_{18}$ chromatographic column, $150 \times 0.3 \mathrm{~mm}$ (Agilent Technologies, Wilmington, DE). The peptides were eluted from the column using an acetonitrile/trifluoroacetic acid gradient (2-72\% acetonitrile in 35 minutes) and spotted directly onto a MALDI plate in 6 second spot fractions. The MALDI spots were analyzed using an ABI 4800 MALDI TOF/TOF analyzer operated with 4000 Series Explorer software. The MS acquisition was in positive ion reflector mode with 400 laser shots per spectrum performed. The 15 strongest precursors per spot were chosen for MS/MS and the MALDI spot was interrogated until at least 4 peaks in the MS/MS, spectra achieved a $S / N \geq 70$.

The resulting MS/MS spectra were analyzed using $A B I$ Protein ProteinPilot software 2.0 (Applied Biosystems, Foster City, CA). The spectral data was searched against the mouse protein database (NCBI nr.fasta database customized to select for all mouse proteins) for identification of the peptides and corresponding proteins. In ProteinPilot, the sample type was selected as ITRAQ 4Plex for retrieval of the isotopic tag information from the mass spectra. After database correlation analysis, the proteins were grouped, scored, and normalized against one to four isotope correction factors. The Pro Group algorithm of ProteinPilot generated a ProtScore that is a cumulative score from each of the peptides used by the algorithm in the protein identification. Protein scores (ProtScore) above 2.0, 1.0, and 0.47 expressed the percent confidence levels of $>99,>90$, and $>66 \%$, respectively. Each peptide match showed the iTRAQ ${ }^{\mathrm{TM}}$ isotopic labels, carbamidomethylated cysteines, and other post-translational modifications present as mass spectral shifts identified during the database correlation analysis. Each protein identified also showed the differential protein expression compared against the other iTRAQ ${ }^{\mathrm{TM}}$ labeled samples for relative quantitation. 


\section{Statistics}

Means and standard errors (SEM) were calculated for all data sets. Data were analyzed with a one-way analysis of variance (ANOVA) method to evaluate the main treatment effect, diabetes induction (GraphPad Software Inc., La Jolla, CA). Fisher's Least Significant Difference (LSD) post hoc tests were performed to determine the significant differences among means. When appropriate a Student's T-test was employed. ${ }^{*} P<0.05$ was considered significant. 


\section{RESULTS}

\section{Db/db Mouse Characterization}

At 18 weeks, body weight was significantly increased (Supplemental data, Table $1 \mathrm{~S}$; $\left.{ }^{*} P<0.05\right)$ in the $\mathrm{db} / \mathrm{db}$ mice as compared to WT mice, which is in agreement with other reports (29). Analyses of fasting glucose levels, circulating free fatty acids and insulin levels revealed significant increases (Supplemental data, Table 4.2; ${ }^{*} P<0.05$ for all three) in the $\mathrm{db} / \mathrm{db}$ mice as compared to WT mice, which is also in agreement with other reports (29). Cardiac contractile function was assessed in isolated perfused hearts. Rates of contraction $(+\mathrm{dP} / \mathrm{d} t)$, relaxation $(-\mathrm{dP} / \mathrm{d} t)$ and developed pressures (DP) were all significantly decreased in $\mathrm{db} / \mathrm{db}$ hearts as compared to WT hearts (Table 4.3; ${ }^{*} P<0.05$ for all three), demonstrating decreased contractility in the $\mathrm{db} / \mathrm{db}$ heart. These data are in agreement with others examining the $\mathrm{db} / \mathrm{db}$ model of type 2 diabetes mellitus (14).

\section{Mitochondrial Subpopulation Morphology}

Forward scatter (FSC), indicative of size, was significantly decreased in the $\mathrm{db} / \mathrm{db}$ SSM compared to WT SSM (Figures $4.1 \mathrm{~A}$ and $4.1 \mathrm{E} ;{ }^{*} P<0.05$ ), yet no significant change in size in the $\mathrm{db} / \mathrm{db}$ IFM as compared to WT IFM was observed (Figures 4.1C and 4.1E). Mitochondrial internal complexity (SSC) was significantly decreased in the $\mathrm{db} / \mathrm{db}$ SSM as compared to WT SSM (Figures 4.1B and 4.1F; ${ }^{*} P<0.05$ ), while $\mathrm{db} / \mathrm{db}$ IFM internal complexity was increased as compared to WT IFM (Figures $4.1 \mathrm{D}$ and 4.1F; $\left.{ }^{*} P<0.05\right)$.

\section{Mitochondrial Subpopulation Functional Assessment}

Assessment of state 3 and state 4 respiration utilizing glutamate/malate revealed a significant decrease in state 3 respiration in $\mathrm{db} / \mathrm{db}$ SSM as compared to WT SSM (Figure 4.2A; ${ }^{*} P<0.05$ ), with no significant changes between WT and $\mathrm{db} / \mathrm{db}$ IFM 
(Figure 4.2B). Further, fatty acid stimulated state 3 and state 4 respiration with palmitoylcarnitine displayed significantly decreased respiration in only $\mathrm{db} / \mathrm{db}$ SSM (Figure 4.2C; ${ }^{*} P<0.05$ ). ETC complexes I, III and IV activities were significantly decreased in $\mathrm{db} / \mathrm{db}$ SSM as compared to WT SSM (Figure 4.2E; ${ }^{*} P<0.05$ for all three), with no significant differences between $\mathrm{db} / \mathrm{db}$ IFM and WT IFM (Figure 4.2F). Finally, ATP synthase activity was significantly decreased in $\mathrm{db} / \mathrm{db}$ SSM as compared to WT SSM (Figure 4.2G; * $P<0.05$ ), with no significant differences between $\mathrm{db} / \mathrm{db}$ and WT IFM (Figure 4.2G).

\section{Mitochondrial Subpopulation Membrane Potential ( $\left.\Delta \Psi_{m}\right)$}

Inner mitochondrial membrane (IMM) potential, orange to green fluorescence ratio, was significantly decreased in the $\mathrm{db} / \mathrm{db}$ SSM as compared to WT SSM (Figures $4.3 \mathrm{~A}-\mathrm{B}$ and $4.3 \mathrm{E} ;{ }^{*} P<0.05$ ) due to a decrease in overall orange fluorescence. No significant differences between $\mathrm{db} / \mathrm{db}$ and WT IFM were observed (Figure 4.3C-D and 4.3E).

\section{Mitochondrial Subpopulation Protein Expression of ANT and UCP3}

Western blot analysis of total ANT protein expression was significantly down in the $\mathrm{db} / \mathrm{db}$ SSM as compared to WT (Figure 4.4A; ${ }^{*} P<0.05$ ), with no significant

differences between $\mathrm{db} / \mathrm{db}$ and WT IFM (Figure 4.4B). In contrast, UCP3 expression was significantly increased in $\mathrm{db} / \mathrm{db}$ SSM as compared to WT (Supplemental data Figure $4.7 ;{ }^{*} P<0.05$ ), with no significant differences between $\mathrm{db} / \mathrm{db}$ and WT IFM (Supplemental data Figure 4.7).

\section{Mitochondrial Subpopulation Oxidative Damage}

MDA and 4-HAE lipid peroxidation by-products were significantly increased in the $\mathrm{db} / \mathrm{db}$ SSM as compared to WT SSM (Figure $4.5 \mathrm{~A}$; ${ }^{*} P<0.05$ ), with no significant changes with $\mathrm{db} / \mathrm{db}$ IFM as compared to WT IFM (Figure 4.5A). Similarly, there was a 
significant increase in 3-nitrotyrosine formation in $\mathrm{db} / \mathrm{db}$ SSM compared to WT (Figure 4.5B; ${ }^{*} P<0.05$ ), with no changes in db/db IFM compared to WT (Figure 4.5B).

\section{Mitochondrial Subpopulation Proteome Analyses}

To determine whether the $\mathrm{db} / \mathrm{db}$ phenotype differentially impacts spatially distinct mitochondrial subpopulation proteomes, we utilized an iTRAQ ${ }^{\mathrm{TM}}$ approach, which enabled simultaneous identification and relative quantification of mitochondrial proteins, through isobaric peptide tagging. Figure 4.6A is an example of an MS/MS fragment ion (spectra) for a peptide that was used to identify a protein, cytochrome c oxidase subunit Vlla1. Figure $4.6 \mathrm{~B}$ is an example of an $\mathrm{iTRAQ}^{\mathrm{TM}}$ tag mass spectral signature $(\mathrm{m} / \mathrm{z} 114$, 115,116 , and 117) used to relatively quantitate cytochrome c oxidase subunit VIla1, within the four labeled sample groups (see figure legend for groups). A second example of the associated $\mathrm{TTRAQ}^{\mathrm{TM}}$ data is included for the protein ubiquinol cytochrome c reductase core protein 2 (Supplemental data; Figures 4.8A-B).

Proteins of Fatty Acid Oxidation. Fatty acid oxidation (FAO) proteins were significantly altered in both the $\mathrm{db} / \mathrm{db}$ SSM and IFM as compared to WT (Table $4.1 ;{ }^{*} P<0.05$ ). However, 5 out of 8 FAO proteins identified were significantly elevated in the $\mathrm{db} / \mathrm{db}$ SSM as compared to WT (Table $4.1 ;{ }^{*} P<0.05$ ). In contrast, 3 of 8 proteins identified were upregulated and 3 of 8 proteins identified were down-regulated in the $\mathrm{db} / \mathrm{db}$ IFM.

Proteins of the Mitochondrial Respiratory Chain. A number of proteins of the mitochondrial respiratory chain were significantly altered in both $\mathrm{db} / \mathrm{db}$ SSM and $\mathrm{db} / \mathrm{db}$ IFM, compared to WT controls. Of the 18 respiratory chain proteins identified, 11 were significantly decreased in the $\mathrm{db} / \mathrm{db}$ SSM as compared to WT, while 8 of 18 respiratory chain proteins identified were increased in expression in the $\mathrm{db} / \mathrm{db}$ IFM compared to WT (Table $4.1 ;{ }^{*} P<0.05$ for all). In the $\mathrm{db} / \mathrm{db}$ SSM, 3 of 12 proteins were from complex I, 2 of 12 were from complex III, 3 of 12 from complex IV and 2 of 12 were from complex V. Western blot analyses of ubiquinol cytochrome c reductase core protein 2 (Figures 4.8 
C-D), a protein of complex III, and cytochrome c oxidase subunit VIla1 (Figures 4.6CD), a protein of complex IV, provided confirmation of the $\mathrm{ITRAQ}^{\mathrm{TM}}$ results observed.

Proteins of the Tricarboxylic Acid Cycle (TCA). Proteins of the TCA cycle were significantly altered in both $\mathrm{db} / \mathrm{db}$ SSM and IFM as compared to WT (Table 4.1; $\left.{ }^{*} P<0.05\right)$. However, only 1 of 4 TCA proteins identified was increased in the $\mathrm{db} / \mathrm{db} S S M$ while 3 of 4 proteins identified were upregulated in the $\mathrm{db} / \mathrm{db}$ IFM as compared to WT. Antioxidant Defense Proteins. Peroxiredoxin V (PRDX5) was the only antioxidant identified in our iTRAQ ${ }^{\mathrm{TM}}$ analyses that was significantly decreased in the $\mathrm{db} / \mathrm{db}$ SSM as compared to WT, while there were no significant changes observed in the IFM antioxidant defense components (Table $4.1 ;{ }^{*} P<0.05$ ).

Mitochondrial Transport Proteins. ANT1 was significantly decreased in the db/db SSM as compared to WT, yet upregulated in the $\mathrm{db} / \mathrm{db}$ IFM (Table $4.1 ;{ }^{*} P<0.05$ ). Western blot analysis confirmed the overall decrease in total ANT expression in the $\mathrm{db} / \mathrm{db} S \mathrm{SSM}$, while total ANT was unchanged in the $\mathrm{db} / \mathrm{db}$ IFM (Figures $4.4 \mathrm{C}$ and $4.4 \mathrm{D}$ ). Glucose regulated protein 75 (Grp75), was significantly decreased in $\mathrm{db} / \mathrm{db}$ SSM as compared to WT (Table 4.1; ${ }^{*} P<0.05$ ), while there were no significant changes in $\mathrm{db} / \mathrm{db}$ IFM compared to WT.

\section{Mitochondrial Subpopulation Post-translational Modifications}

Fifteen proteins presented with some type of PTM in the $\mathrm{db} / \mathrm{db}$ SSM that were non-existent in the WT SSM, while 10 proteins were present in the $\mathrm{db} / \mathrm{db}$ IFM (Table 4.5). Seven of 15 proteins identified were of the ETC and they included specific PTM's, such as acetylations, deamidations and oxidations. Interestingly, 3 of 10 proteins identified in the $\mathrm{db} / \mathrm{db}$ IFM were of the ETC and they included modifications such as acetylations, deamidations and methylations. Of greatest interest was that the majority of the modified proteins were IMM proteins, suggesting that this locus may play a key role in the pathogenesis of cardiac dysfunction associated with type 2 diabetes mellitus. 


\section{DISCUSSION}

Mitochondrial dysfunction is a major contributor to the development of various pathologies, including type 1 and type 2 diabetes mellitus $(18,24,25,35,37,38)$. Nevertheless, mitochondrial analyses are complicated by the fact that two spatially and functionally distinct populations of mitochondria exist in the cardiomyocyte, SSM and IFM. We and others have shown that these two mitochondrial populations respond differently to pathological insults $(12,25,27,30,37)$. The goal of this study was to determine whether subcellular location was associated with differential impact on cardiac mitochondria in a type 2 diabetes mellitus model, the $\mathrm{db} / \mathrm{db}$ mouse. Our results indicate that SSM, located below the sarcolemma, display greater dysfunctional profiles as compared to IFM, which are situated between the myofibrils. These dysfunctional profiles are the result of differential impact on a number of mitochondrial indices including structure, function, oxidative milieu, and proteome make-up.

Morphological alterations have been associated with mitochondrial dysfunction $(17,24,37,44)$. Kelley et al. reported a decrease in mitochondrial size and a decrease in the abundance of SSM from skeletal muscle of type 2 diabetic patients, while we show a decrease in size and complexity of cardiac SSM. Taken together these studies suggest that type 2 diabetes mellitus imparts a specific influence on SSM structure. Interestingly, we observed a significant increase in IFM complexity (Figure 4.1). It is unclear as to why such a phenomenon occurred, and may be the result of an increased abundance of ETC components in db/db IFM which we observed in our proteome analyses (Table 4.1). Regardless, the result is interesting and warrants further investigation.

Studies suggest that mitochondrial respiration is significantly altered in the $\mathrm{db} / \mathrm{db}$

heart. However, these analyses have focused on total mitochondria $(3,4)$. In the current study, we observed decreases in state 3 respiration and ETC complex activities I, III, and IV, exclusively in db/db SSM (Figure 4.2). Our results are in agreement with others reporting decreases in succinate-driven respiration of SSM from vastus lateralis of type 2 diabetic patients (37). Additionally, we observed a significant decrease in ATP synthase activity solely in the $\mathrm{db} / \mathrm{db}$ SSM. These findings indicate that the ability for 
$\mathrm{db} / \mathrm{db}$ SSM to generate ATP may be compromised, thus, those processes that have been suggested by others, to rely upon ATP from this source, such as signal transduction pathways, and substrate transport across the plasma membrane, may be specifically impacted (37). Further, we observed a significant decrease in $\Delta \Psi_{\mathrm{m}}$ in $\mathrm{db} / \mathrm{db}$ SSM with no change in IFM (Fig. 4.3). Alterations to $\Delta \Psi_{\mathrm{m}}$ have been indicated to impact $F_{1} F_{0}$ ATP synthase function (2), and as a result, may have contributed to the diminished function observed in $\mathrm{db} / \mathrm{db}$ SSM. ANT, which catalyzes the exchange of ADP and ATP across the IMM, was significantly decreased only in db/db SSM (Fig. 4.4A). This observation was confirmed by the iTRAQ ${ }^{T M}$ analyses in which ANT1 was significantly decreased in $\mathrm{db} / \mathrm{db}$ SSM yet increased in $\mathrm{db} / \mathrm{db}$ IFM. The increase in ANT in the IFM was not observed in the Western blot analyses, which may be due to the fact that total ANT was assessed and not ANT1. Inhibition of mitochondrial ANT has been proposed to contribute to cellular dysfunction in obesity and type 2 diabetes mellitus by increasing reactive oxygen species and adenosine (10). Our finding of decreased ANT expression is in agreement with other reports (3), and supports the hypothesis that decreases in ANT expression may contribute to a reduction in the ability to generate ATP, which was also observed (Fig. 4.3).

Mitochondrial oxidative stress has been reported in models of both type 1 (12, $18,38,44)$ and type $2(3,37)$ diabetes mellitus, and may play a central role in mitochondrial dysfunction. In the current study lipid peroxidation and protein nitration products were increased only in $\mathrm{db} / \mathrm{db}$ SSM (Fig. 4.5), suggesting that type 2 diabetes mellitus presents an enhanced oxidative environment solely to the SSM. Increased reactive oxygen species production and oxidative stress by-products in a type 2 diabetic model has been reported previously (3), and suggested to impact submitochondrial components, particularly in the IMM. Enhanced reactive oxygen species production and lipid peroxidation by-products can disrupt the fluidity and integrity of the IMM and further modify protein and lipid constituents within the membrane altering overall mitochondrial function (9). Further, db/db SSM displayed decreases in PRDX5, an antioxidant that reduces hydrogen peroxide and alkyl hydroperoxides. Loss of PRDX5 may have contributed to the enhanced oxidative environment observed in this particular population of mitochondria. Interestingly, PRDX5 was the only major antioxidant significantly 
decreased in $\mathrm{db} / \mathrm{db}$ SSM. The result suggests that loss of PRDX5 may contribute to the enhanced oxidative milieu observed in $\mathrm{db} / \mathrm{db}$ SSM. It is important to point out that other mechanisms may have also been involved in the increased oxidative stress observed in $\mathrm{db} / \mathrm{db}$ SSM, such as accumulation of neutral fatty acids which are susceptible to oxidation $(3,40)$.

Several groups have identified mitochondrial proteomic alterations with various type 1 diabetic models $(6,11,20,44,47)$. However, no examination of spatially distinct mitochondrial subpopulation profiles has been performed in any pathological model, including diabetes mellitus. Kavazis et al. examined the impact of exercise training on cardiac SSM and IFM proteomes and observed subpopulation-specific changes (23). We examined the impact of type 2 diabetic insult on spatially distinct cardiac mitochondrial proteomes. The use of ITRAQ $^{\mathrm{TM}}$ isobaric labeling technology coupled with MudPIT-based mass spectral analyses of the labeled samples provides significant advantages over previously used methods (2-dimensional (2D) gel electrophoresis, 2DDIGE and label-free UPLC-MS). These advantages include global protein identification and characterization of the complex samples, simple and robust isotopic labeling that does not bias analytical separations, and simultaneous identification and relative quantification of the proteomes of the four samples. Using the $\mathrm{iTRAQ}{ }^{\mathrm{TM}}$ approach, we observed an increase in FAO proteins in both SSM and IFM of $\mathrm{db} / \mathrm{db}$ hearts (Table 4.1), which is probably due, in part, to the enhanced lipid environment present with type 2 diabetes mellitus (1). However, though the effect occurred in both subpopulations, the result was more pronounced in the SSM, which saw a greater number of FAO proteins increased in abundance, suggesting a more enhanced fatty acid environment presented to this subpopulation. We also observed an increase in circulating free fatty acid levels (Supplemental data Table 4.2). Because SSM are located at the periphery of the cell, they may be impacted to a greater extent by an enhanced lipid environment, as compared to IFM, which reside in the interior of the cell. Nielson et al. observed lipid accumulation specifically surrounding the SSM in type 2 diabetic skeletal muscle suggesting that the SSM may be subject to a more enhanced lipid environment in the type 2 diabetic state based upon its proximity (31). In addition, we observed increases in UCP3 protein expression in db/db SSM (Supplemental data Figure 4.7), which is in 
agreement with other authors (41). Because UCP3 has been suggested to be upregulated in the presence of enhanced free fatty acids, its expression may be indicative of a greater fatty acid environment in the $\mathrm{db} / \mathrm{db}$ SSM. However, though we observed upregulation of FAO proteins in the SSM, the ability to utilize fatty acid substrates (palmitoylcarnitine) was diminished in the $\mathrm{db} / \mathrm{db}$ SSM (Figure 2), suggesting that the mitochondrial dysfunction displayed by $\mathrm{db} / \mathrm{db}$ SSM at 18 weeks may be due to limitations to the ETC as opposed to FAO.

In general, ETC complex proteins were primarily decreased in $\mathrm{db} / \mathrm{db}$ SSM, while ETC and TCA cycle proteins were increased in the IFM. The changes observed in $\mathrm{db} / \mathrm{db}$ SSM were not specific to one ETC complex indicating that both proximal and distal portions of the respiratory chain were impacted. These results indicate that protein loss in the SSM may be contributing to the decrease in oxidative capacity observed in the SSM. Further, the majority of the proteins lost in the $\mathrm{db} / \mathrm{db}$ SSM reside in the IMM, including ETC proteins, which may have contributed to the decrease in oxidative capacity observed in the SSM. Taken together, these results suggest that the formation and/or organization of ETC constituents may have been impacted to a greater extent in $\mathrm{db} / \mathrm{db}$ SSM which may have contributed to the respiratory dysfunction observed. In contrast, preservation of proteins involved in mitochondrial bioenergetics in $\mathrm{db} / \mathrm{db}$ IFM may have been sufficient to prevent loss of respiratory capacity.

Our proteomic analyses revealed changes to a number of other key mitochondrial proteins (Table 4.1). Several proteins involved in protein/substrate import necessary for proper mitochondrial function were significantly altered by type 2 diabetes mellitus. Grp75, a mitochondrial chaperone and the active motor component of the mitochondrial import process, was significantly decreased only in $\mathrm{db} / \mathrm{db}$ SSM which may have implications for the nuclear-encoded mitochondrial protein import process. Since the vast majority of mitochondrial proteins are encoded by nuclear sources, such a finding may have repercussions for a number of essential mitochondrial functions, including structure, function and antioxidant capacity.

Because assessment of abundance does not entirely account for protein functionality, we examined PTM in the $\mathrm{db} / \mathrm{db}$ heart of mitochondrial subpopulations. Though both mitochondrial subpopulations saw increased PTM as a result of type 2 
diabetic insult, the result was more pronounced in the SSM, which saw a greater number of PTMs (Table 4.5). Of particular interest was the observation that ETC proteins displayed oxidations at specific amino acid residues, and this phenomenon occurred only in $\mathrm{db} / \mathrm{db}$ SSM. Oxidation to these proteins found within the ETC may alter the structure and/or function of each protein and subsequently contribute to the diminished respiratory capacity reported. As a result, this may compromise the SSM ability to provide adequate ATP for cellular processes (Figure 4.2). Additionally, our findings suggest that IFM show less protein modifications and protein loss in comparison with SSM, suggesting a potential adaptive response to prevent diabetesinduced mitochondrial dysfunction. It should be noted that though the MudPIT-based mass spectral method provides a wealth of global protein identification and characterization information, the larger number of peptides analyzed from an ultracomplex sample can mask those species containing PTMs, due to the greater natural abundance and higher ionization efficiencies of the non-modified forms. Other methods exist for assessing PTMs which may utilize selective enrichment strategies or enhanced sensitivity, thus additional studies may provide complementary information (15).

The most interesting finding in the current study was that in a type 2 diabetic model, cardiac SSM are affected to a greater extent by the pathology as compared to cardiac IFM. These results are in contrast with our previous findings indicating that in a type 1 diabetic model (streptozotocin) the IFM are most impacted by the pathology. These findings are probably due to differences in the phenotypes elicited by the two conditions, resulting from the unique pathological etiologies specific for each model. For example, higher free fatty acids and lipid deposition associated with the subsarcolemmal region during type 2 diabetes mellitus may put the SSM at greater risk due to their location at the cell periphery as compared to IFM (32). It is plausible that an increased lipid environment in and surrounding the SSM may enhance the oxidative environment within the SSM contributing to their dysfunction. Such a phenomenon would manifest during type 2 diabetes mellitus to a greater extent with an enhanced lipid environment as compared to type 1 diabetes mellitus. Regardless, it is important to point out that in multiple models of both type 1 diabetes mellitus $(12,22,43)$ and type 2 
diabetes mellitus (4) oxidative stress appears to be an underlying contributor to mitochondrial dysfunction. However, the mechanism in which enhanced oxidative stress manifests may be different between the type 1 and type 2 diabetic heart. Further, it is important to point out that diminished function in either subpopulation may have negative implications on cardiac contraction regardless of subpopulation. Though it has been speculated that each subpopulation provides ATP for specific cellular functions, inability to provide ATP for any of these functions may translate into contractile abnormalities in the heart.

In conclusion, mitochondrial dysfunction in the type 2 diabetic heart is confined primarily to those mitochondria residing below the plasma membrane, SSM with minimal effect on those mitochondria situated between the myofibrils, IFM. Of particular interest is the observation that proteins of the IMM, an important locus containing the ETC, ATP synthesis machinery, and protein trafficking constituents, were specifically impacted in the SSM as a result of type 2 diabetes mellitus. Functional aspects of the IMM, such as $\Delta \Psi_{\mathrm{m}}$, respiration, ETC activities, and ATP synthesis were concomitantly diminished in the SSM. Taken together, the combination of findings provided by our study offer evidence that not all cardiac mitochondria are impacted equally by type 2 diabetic insult, but rather mitochondrial dysfunction is specific to a particular subcellular locale. Our analyses of mitochondrial subpopulation proteomic compositional changes resulting from type 2 diabetic insult reveals submitochondrial loci at risk, and identifies specific proteomic targets that may be of interest for therapeutic and diagnostic interventions. 


\section{ACKNOWLEDGEMENTS}

We would like to thank Dr. Christopher Cuff and the contributions from the West Virginia University Flow Cytometry Core facility. This work was supported by the National Institutes of Health from the National Institutes of Diabetes and Digestive and Kidney Diseases [DP2DK083095]. This work was also supported by a Grant-In-Aid from the American Heart Association [0855484D]. Erinne Dabkowski is a recipient of an American Heart Association Predoctoral Fellowship [0815406D]. Flow cytometry studies were supported in part by grants from the National Institutes of Health [RR020866 and RR16440]. 


\section{REFERENCES}

1. Abdul-Ghani MA, Muller FL, Liu Y, Chavez AO, Balas B, Zuo P, Chang Z, Tripathy D, Jani R, Molina-Carrion M, Monroy A, Folli F, Van Remmen H, and DeFronzo RA. Deleterious action of FA metabolites on ATP synthesis: possible link between lipotoxicity, mitochondrial dysfunction, and insulin resistance. Am J Physiol Endocrinol Metab 295: E678-685, 2008.

2. Bornhovd C, Vogel F, Neupert W, and Reichert AS. Mitochondrial membrane potential is dependent on the oligomeric state of F1F0-ATP synthase supracomplexes. J Biol Chem 281: 13990-13998, 2006.

3. Boudina S, and Abel ED. Mitochondrial uncoupling: a key contributor to reduced cardiac efficiency in diabetes. Physiology (Bethesda) 21: 250-258, 2006.

4. Boudina S, Sena S, Theobald H, Sheng X, Wright JJ, Hu XX, Aziz S, Johnson JI, Bugger H, Zaha VG, and Abel ED. Mitochondrial energetics in the heart in obesity-related diabetes: direct evidence for increased uncoupled respiration and activation of uncoupling proteins. Diabetes 56: 2457-2466, 2007.

5. Bradford MM. A rapid and sensitive method for the quantitation of microgram quantities of protein utilizing the principle of protein-dye binding. Analytical biochemistry 72: 248-254, 1976.

6. Bugger H, Chen D, Riehle C, Soto J, Theobald HA, Hu XX, Ganesan B, Weimer BC, and Abel ED. Tissue-specific remodeling of the mitochondrial proteome in type 1 diabetic akita mice. Diabetes 58: 1986-1997, 2009.

7. Chance B, and Williams GR. Respiratory enzymes in oxidative phosphorylation. I. Kinetics of oxygen utilization. J Biol Chem 217: 383-393, 1955.

8. Chance B, and Williams GR. Respiratory enzymes in oxidative phosphorylation. VI. The effects of adenosine diphosphate on azide-treated mitochondria. J Biol Chem 221: 477-489, 1956.

9. Chen JJ, and Yu BP. Alterations in mitochondrial membrane fluidity by lipid peroxidation products. Free Radic Biol Med 17: 411-418, 1994.

10. Ciapaite J, Bakker SJ, Diamant M, van Eikenhorst G, Heine RJ, Westerhoff HV, and Krab K. Metabolic control of mitochondrial properties by adenine nucleotide 
translocator determines palmitoyl-CoA effects. Implications for a mechanism linking obesity and type 2 diabetes. FEBS J 273: 5288-5302, 2006.

11. Dabkowski E. Quantitative proteomic analysis of distinct mitochondrial subpopulations in diabetic myocardium

Faseb J 1226.36: 2008.

12. Dabkowski ER, Williamson CL, Bukowski VC, Chapman RS, Leonard SS, Peer CJ, Callery PS, and Hollander JM. Diabetic cardiomyopathy-associated dysfunction in spatially distinct mitochondrial subpopulations. Am J Physiol Heart Circ Physiol 296: H359-369, 2009.

13. Dabkowski ER, Williamson CL, and Hollander JM. Mitochondria-specific transgenic overexpression of phospholipid hydroperoxide glutathione peroxidase (GPx4) attenuates ischemia/reperfusion-associated cardiac dysfunction. Free Radic Biol Med 45: 855-865, 2008.

14. Daniels A, van Bilsen M, Janssen BJ, Brouns AE, Cleutjens JP, Roemen TH, Schaart G, van der Velden J, van der Vusse GJ, and van Nieuwenhoven FA. Impaired cardiac functional reserve in type 2 diabetic $\mathrm{db} / \mathrm{db}$ mice is associated with metabolic, but not structural, remodelling. Acta Physiol (Oxf) 2010.

15. Desiderio DaN, NM editor. Redox Proteomics. Hoboken, New Jersey: John Wiley and Sons, Inc., Publications, 2006, p. 939.

16. Feniouk BA, Suzuki T, and Yoshida M. Regulatory interplay between proton motive force, ADP, phosphate, and subunit epsilon in bacterial ATP synthase. $J$ Biol Chem 282: 764-772, 2007.

17. Figueiredo PA, Ferreira RM, Appell HJ, and Duarte JA. Age-induced morphological, biochemical, and functional alterations in isolated mitochondria from murine skeletal muscle. J Gerontol A Biol Sci Med Sci 63: 350-359, 2008.

18. Flarsheim CE, Grupp IL, and Matlib MA. Mitochondrial dysfunction accompanies diastolic dysfunction in diabetic rat heart. The American journal of physiology 271: H192-202, 1996.

19. Garcia MJ, McNamara PM, Gordon T, and Kannel WB. Morbidity and mortality in diabetics in the Framingham population. Sixteen year follow-up study. Diabetes 23: 105-111, 1974. 
20. Hamblin M, Friedman DB, Hill S, Caprioli RM, Smith HM, and Hill MF. Alterations in the diabetic myocardial proteome coupled with increased myocardial oxidative stress underlies diabetic cardiomyopathy. J Mol Cell Cardiol 42: 884-895, 2007.

21. Hofhaus G, Shakeley RM, and Attardi G. Use of polarography to detect respiration defects in cell cultures. Methods in enzymology 264: 476-483, 1996.

22. Kaul N, Siveski-lliskovic N, Thomas TP, Hill M, Khaper N, and Singal PK. Probucol improves antioxidant activity and modulates development of diabetic cardiomyopathy. Nutrition 11: 551-554, 1995.

23. Kavazis AN, Alvarez S, Talbert E, Lee $\mathbf{Y}$, and Powers SK. Exercise training induces a cardioprotective phenotype and alterations in cardiac subsarcolemmal and intermyofibrillar mitochondrial proteins. Am J Physiol Heart Circ Physiol 297: H144-152, 2009.

24. Kelley DE, He J, Menshikova EV, and Ritov VB. Dysfunction of mitochondria in human skeletal muscle in type 2 diabetes. Diabetes 51: 2944-2950, 2002.

25. King KL, Young ME, Kerner J, Huang H, O'Shea KM, Alexson SE, Hoppel CL, and Stanley WC. Diabetes or peroxisome proliferator-activated receptor alpha agonist increases mitochondrial thioesterase I activity in heart. Journal of lipid research 48: 1511-1517, 2007.

26. Laemmli UK. Cleavage of structural proteins during the assembly of the head of bacteriophage T4. Nature 227: 680-685, 1970.

27. Lesnefsky EJ, Slabe TJ, Stoll MS, Minkler PE, and Hoppel CL. Myocardial ischemia selectively depletes cardiolipin in rabbit heart subsarcolemmal mitochondria. American journal of physiology 280: H2770-2778, 2001.

28. Lin D. Multidimensional Protein Identification Technology as an Effective Tool for Proteomics. American Genomic/Proteomic Technology 1: 38-46, 2001.

29. Masaki Y, Atsuhiko Kato, Chie Kato, Etsuko Fujii, Kenji Adachi, Akio Miyoshi and Masami Suzuki Segmentation of the Pathophysiological Stages of Diabetic Changes in the db/db Mouse. Journal of Toxicologic Pathology ～22: 2009. 
30. Mollica MP, Lionetti L, Crescenzo R, D'Andrea E, Ferraro M, Liverini G, and lossa S. Heterogeneous bioenergetic behaviour of subsarcolemmal and intermyofibrillar mitochondria in fed and fasted rats. Cell Mol Life Sci 63: 358-366, 2006.

31. Nielsen J, Mogensen M, Vind BF, Sahlin K, Hojlund K, Schroder HD, and Ortenblad N. Increased subsarcolemmal lipids in type 2 diabetes: effect of training on localization of lipids, mitochondria, and glycogen in sedentary human skeletal muscle. Am J Physiol Endocrinol Metab 298: E706-713.

32. Nielsen J, Mogensen M, Vind BF, Sahlin K, Hojlund K, Schroder HD, and Ortenblad N. Increased subsarcolemmal lipids in type 2 diabetes: effect of training on localization of lipids, mitochondria, and glycogen in sedentary human skeletal muscle. Am J Physiol Endocrinol Metab 298: E706-713, 2010.

33. Palmer JW, Tandler B, and Hoppel CL. Biochemical properties of subsarcolemmal and interfibrillar mitochondria isolated from rat cardiac muscle. $\mathrm{J} \mathrm{Biol}$ Chem 252: 8731-8739, 1977.

34. Pullman ME, Penefsky HS, Datta A, and Racker E. Partial resolution of the enzymes catalyzing oxidative phosphorylation. I. Purification and properties of soluble dinitrophenol-stimulated adenosine triphosphatase. J Biol Chem 235: 3322-3329, 1960.

35. Rabol R, Boushel $\mathbf{R}$, and Dela $\mathbf{F}$. Mitochondrial oxidative function and type 2 diabetes. Applied physiology, nutrition, and metabolism = Physiologie appliquee, nutrition et metabolisme 31: 675-683, 2006.

36. Reers M, Smith TW, and Chen LB. J-aggregate formation of a carbocyanine as a quantitative fluorescent indicator of membrane potential. Biochemistry 30: 4480-4486, 1991.

37. Ritov VB, Menshikova EV, He J, Ferrell RE, Goodpaster BH, and Kelley DE. Deficiency of subsarcolemmal mitochondria in obesity and type 2 diabetes. Diabetes 54 : 8-14, 2005.

38. Rolo AP, and Palmeira CM. Diabetes and mitochondrial function: role of hyperglycemia and oxidative stress. Toxicology and applied pharmacology 212: 167178, 2006.

39. Rosca MG, Okere IA, Sharma N, Stanley WC, Recchia FA, and Hoppel CL. Altered expression of the adenine nucleotide translocase isoforms and decreased ATP 
synthase activity in skeletal muscle mitochondria in heart failure. $\mathrm{J} \mathrm{Mol} \mathrm{Cell} \mathrm{Cardiol} \mathrm{46:}$ 927-935, 2009.

40. Russell AP, Gastaldi G, Bobbioni-Harsch E, Arboit P, Gobelet C, Deriaz O, Golay A, Witztum JL, and Giacobino JP. Lipid peroxidation in skeletal muscle of obese as compared to endurance-trained humans: a case of good vs. bad lipids? FEBS Lett 551: 104-106, 2003.

41. Schrauwen P, and Hesselink MK. Oxidative capacity, lipotoxicity, and mitochondrial damage in type 2 diabetes. Diabetes 53: 1412-1417, 2004.

42. Semeniuk LM, Kryski AJ, and Severson DL. Echocardiographic assessment of cardiac function in diabetic $\mathrm{db} / \mathrm{db}$ and transgenic $\mathrm{db} / \mathrm{db}$-hGLUT4 mice. Am J Physiol Heart Circ Physiol 283: H976-982, 2002.

43. Shen X, Ye G, Metreveli NS, and Epstein PN. Cardiomyocyte defects in diabetic models and protection with cardiac-targeted transgenes. Methods Mol Med 112: 379-388, 2005.

44. Shen X, Zheng S, Thongboonkerd V, Xu M, Pierce WM, Jr., Klein JB, and Epstein PN. Cardiac mitochondrial damage and biogenesis in a chronic model of type 1 diabetes. Am J Physiol Endocrinol Metab 287: E896-905, 2004.

45. Taegtmeyer $\mathbf{H}$, McNulty $\mathbf{P}$, and Young ME. Adaptation and maladaptation of the heart in diabetes: Part I: general concepts. Circulation 105: 1727-1733, 2002.

46. Trounce IA, Kim YL, Jun AS, and Wallace DC. Assessment of mitochondrial oxidative phosphorylation in patient muscle biopsies, lymphoblasts, and transmitochondrial cell lines. Methods in enzymology 264: 484-509, 1996.

47. Turko IV, and Murad F. Quantitative protein profiling in heart mitochondria from diabetic rats. J Biol Chem 278: 35844-35849, 2003. 
Figure 4.1

A

\section{SSM}
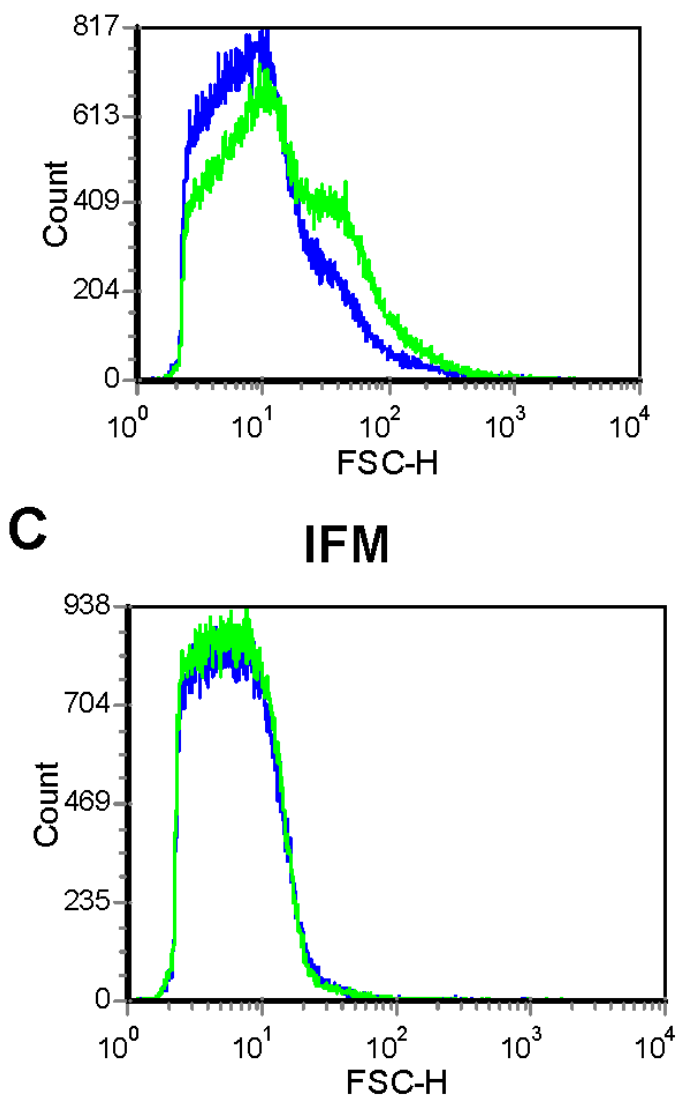

B
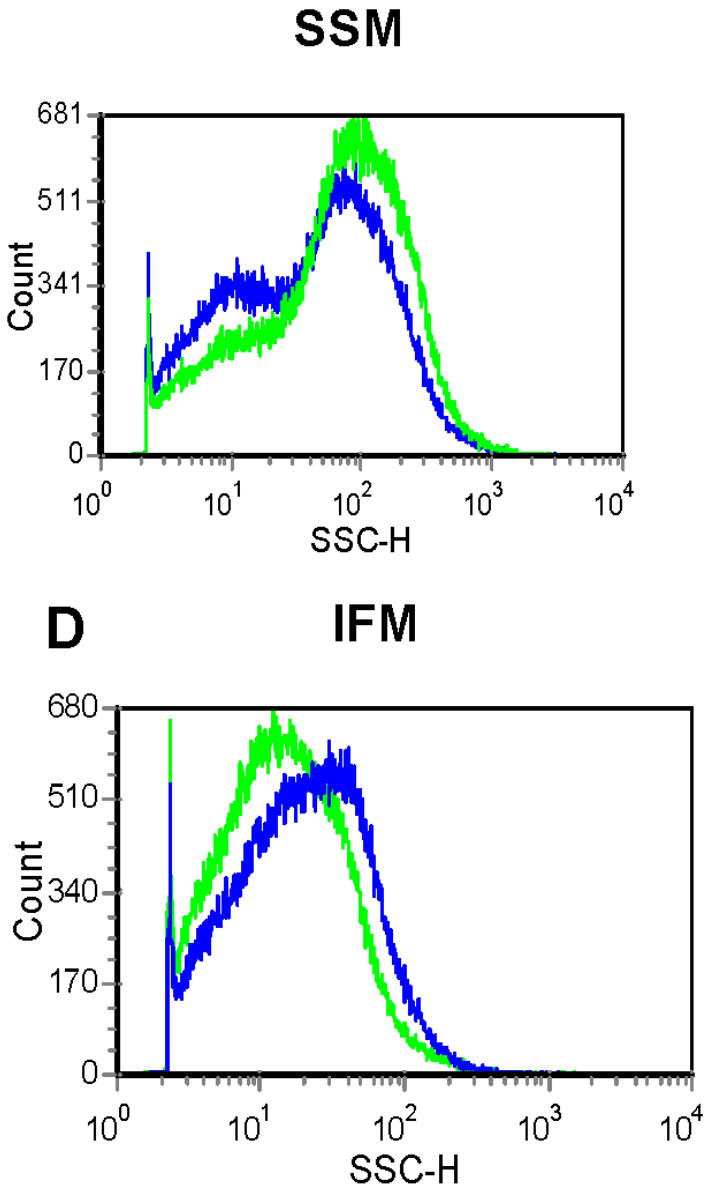
Figure 4.1

E Size

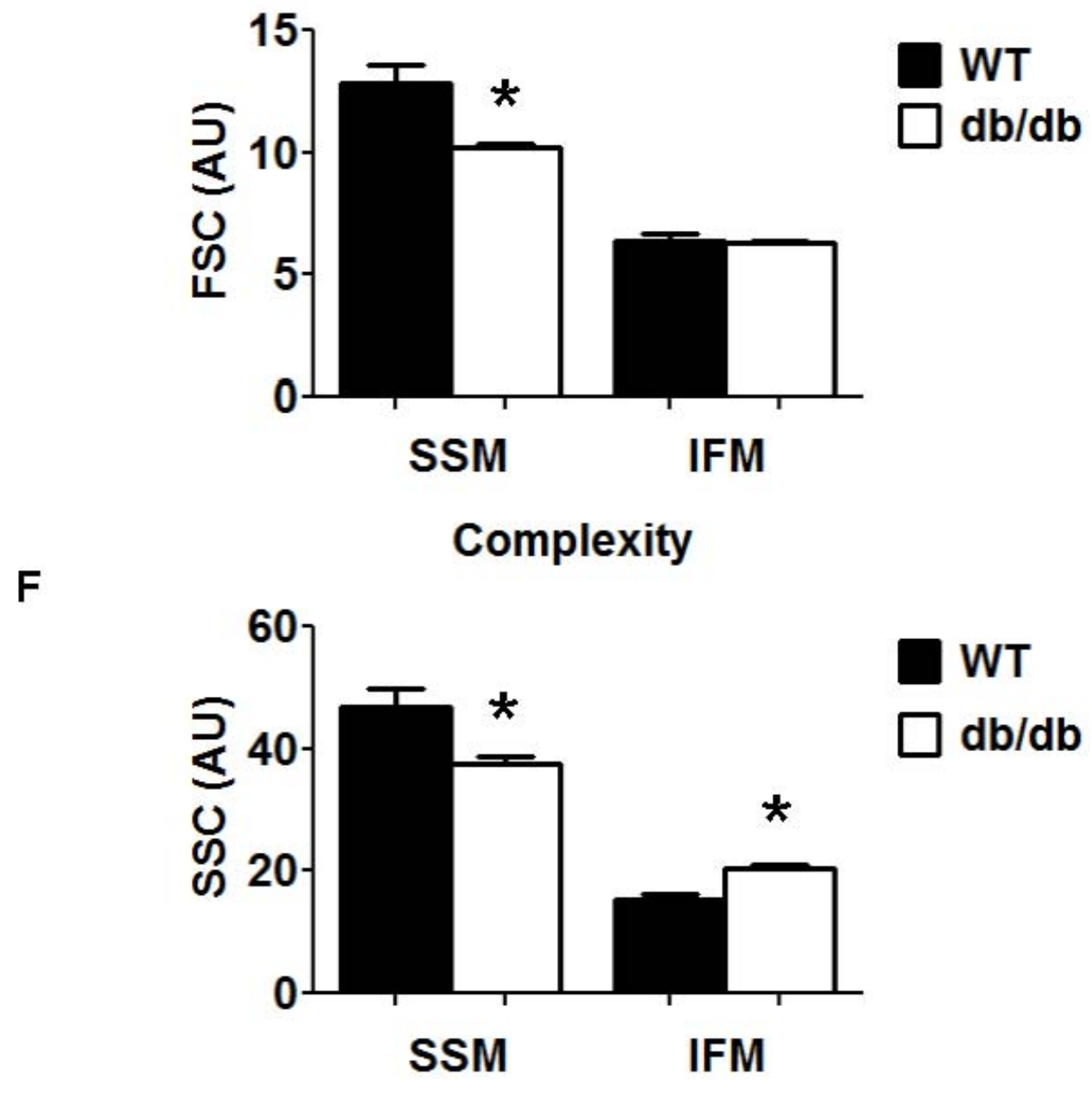


Figure 4.1. Mitochondrial subpopulation size (FSC) and internal complexity (SSC) in WT and db/db hearts. Determination of relative size and internal complexity of distinct mitochondrial subpopulations using flow cytometric analyses. Mitochondrial subpopulations were stained with mitotracker deep Red 633, a membrane potential dependent dye, and gated based on incorporation of the dye. Analysis of FSC and SSC were calculated per 20,000 gated events for all mitochondrial subpopulations. Representative histograms of FSC (A, C) and SSC (B, D) in WT SSM and IFM (green) vs. db/db SSM and IFM (blue). Values for FSC (E) and SSC $(F)$ are expressed as arbitrary units $(A U) \pm S E M ; N=4$ for each group. ${ }^{*} P<0.05$ for WT vs. $\mathrm{db} / \mathrm{db}$. 
Figure 4.2

A

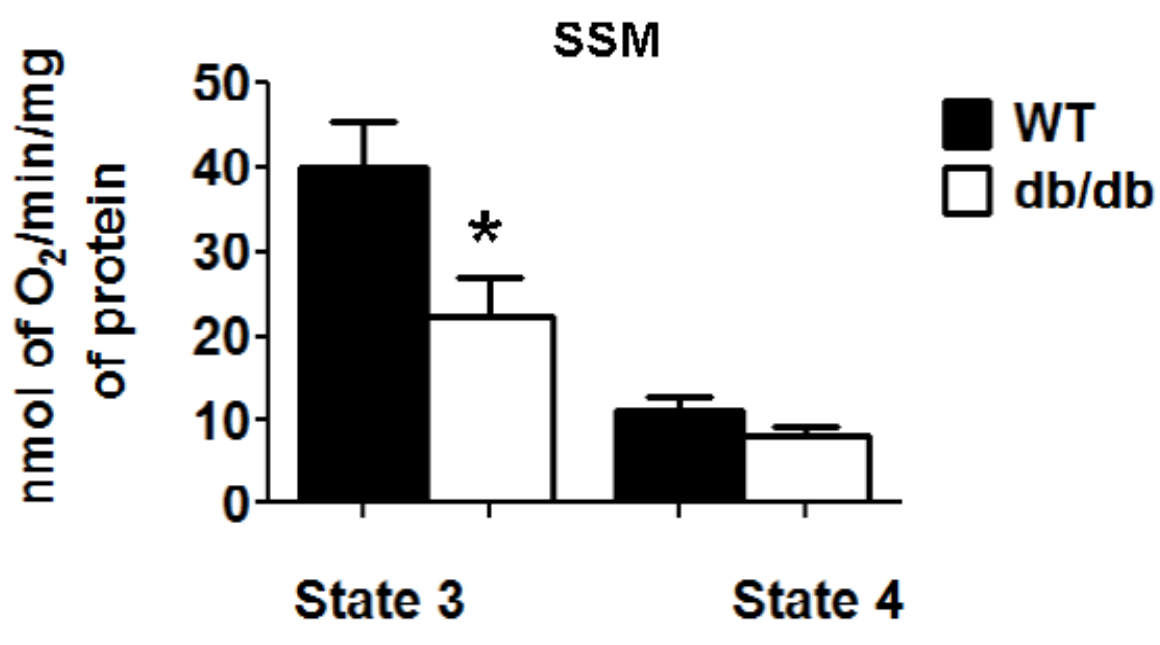

B

IFM

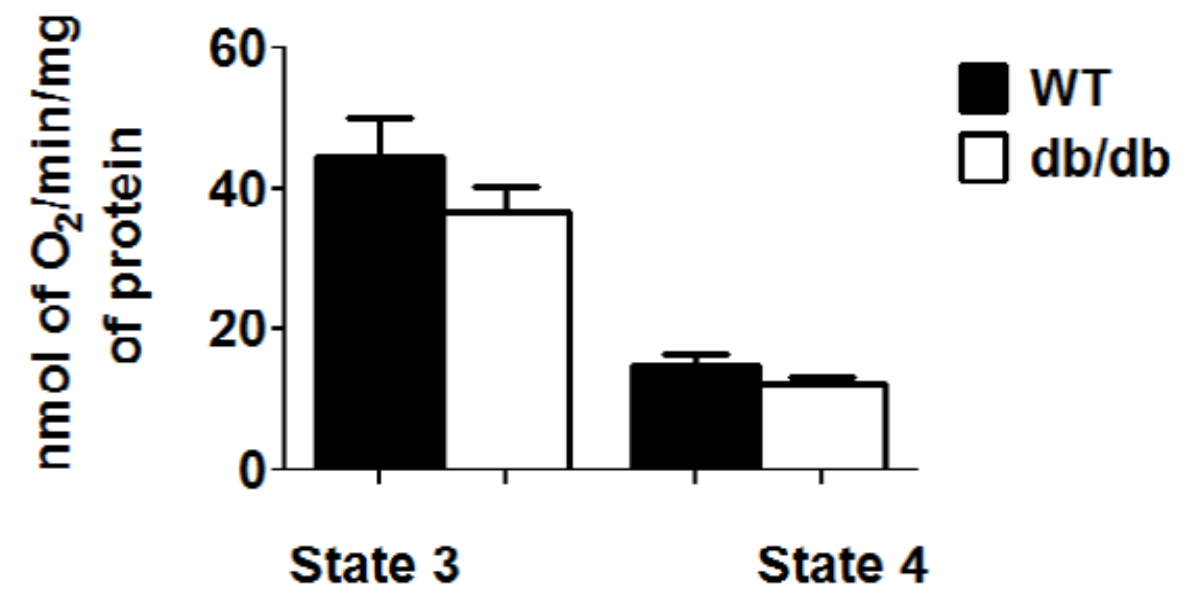


Figure 4.2

C

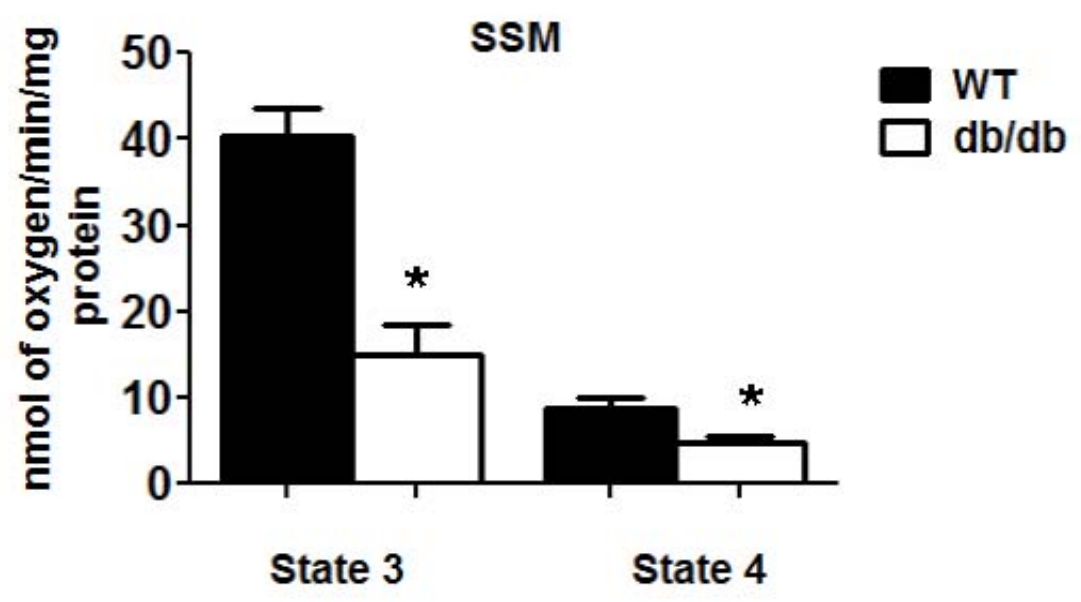

IFM

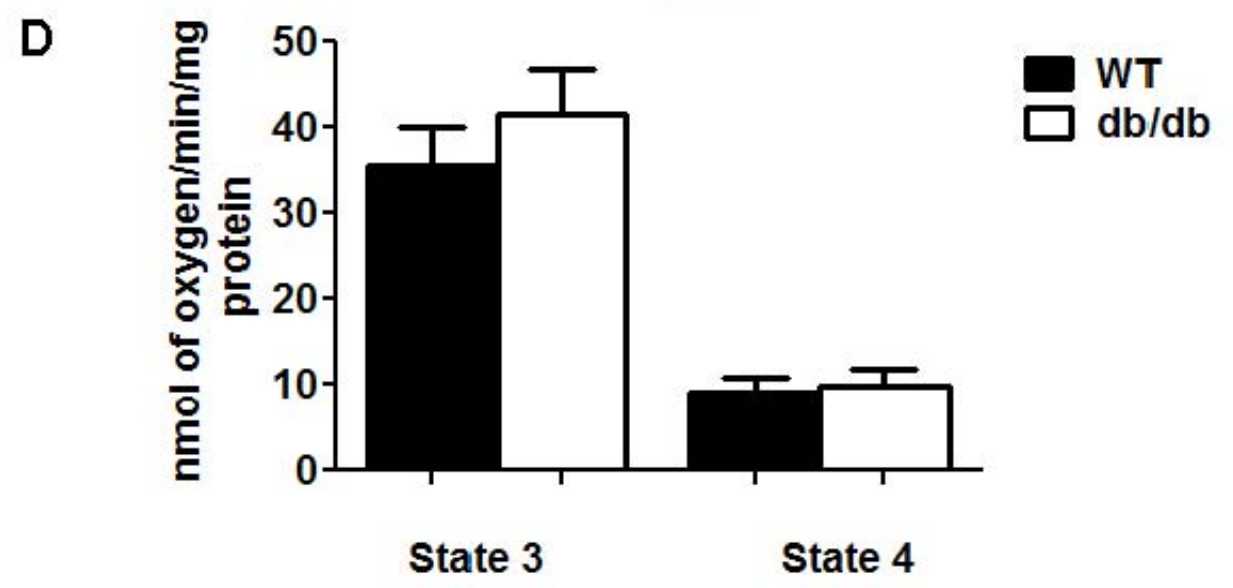


Figure 4.2

E

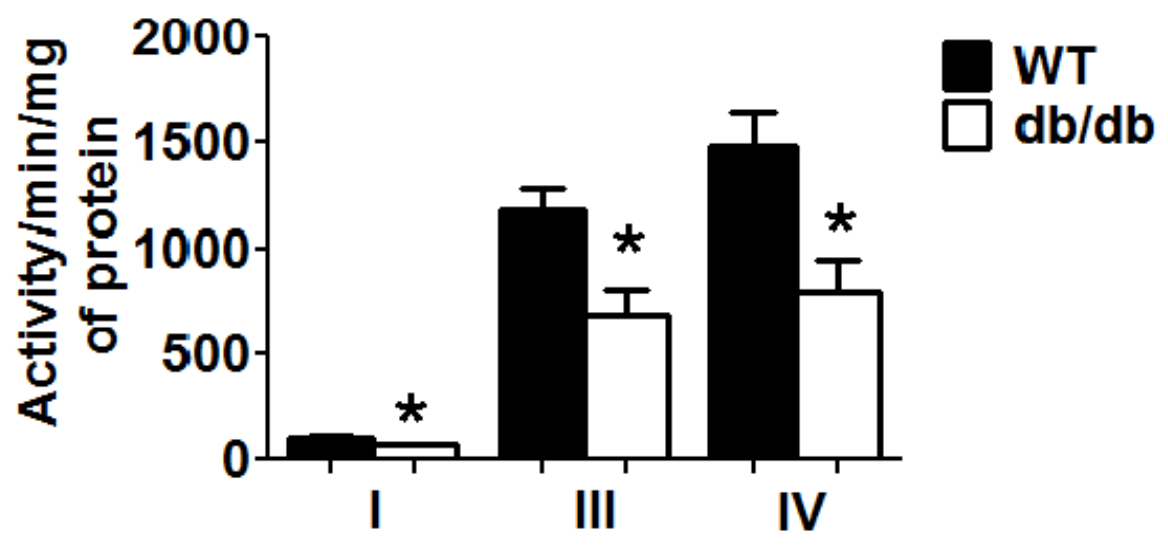

F

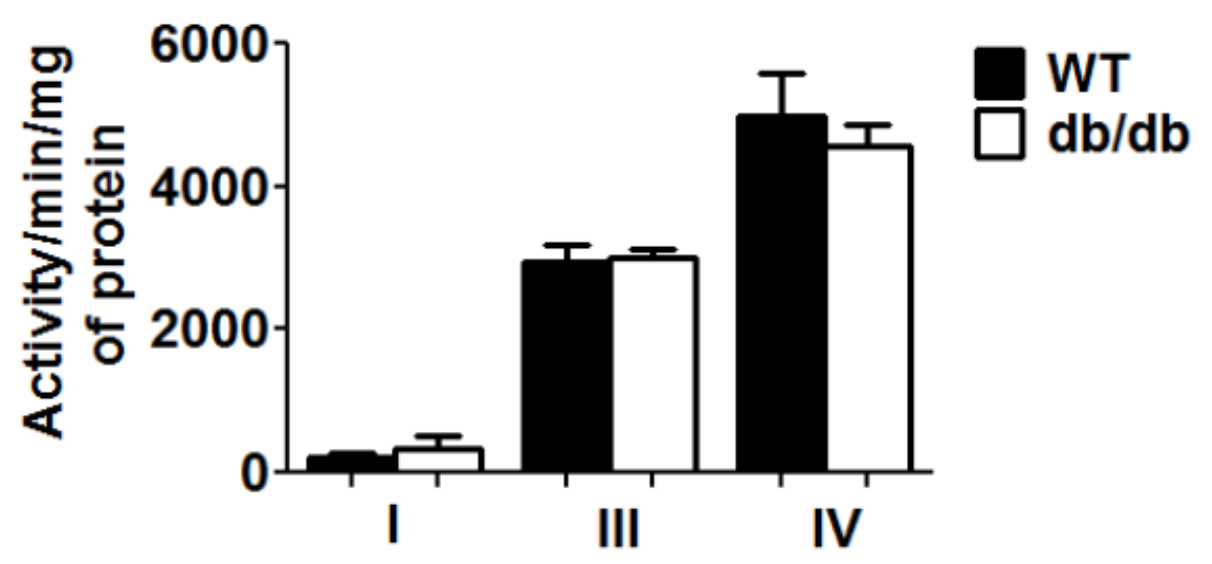


Figure 4.2

G

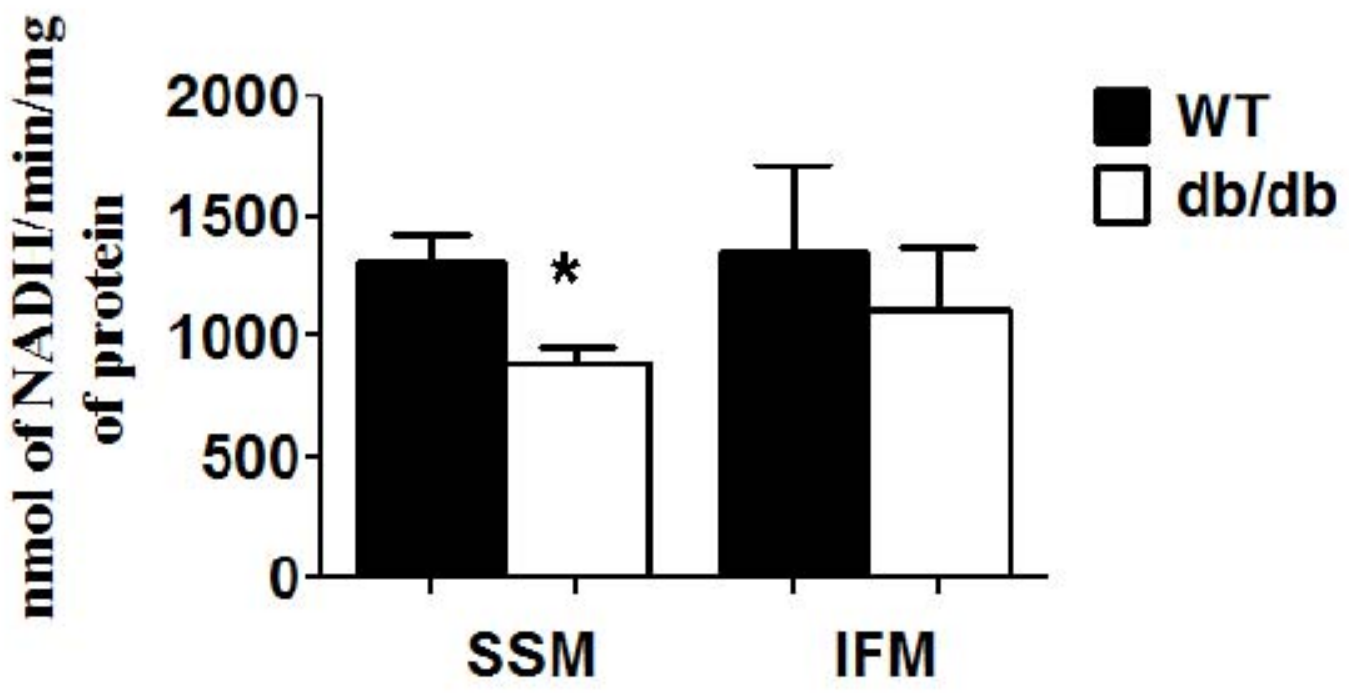


Figure 4.2. Respiratory capacity of mitochondrial subpopulations in $\mathbf{W T}$ and $\mathbf{d b} / \mathbf{d b}$ hearts. State 3 and state 4 respiration rates with glutamate/malate (A, B), palmitoylcarnitine (C, D), ETC complex activities $(E, F)$ and ATP synthase activity $(G)$ were assessed in isolated mitochondrial subpopulations (SSM; A, C and E) and (IFM; B, D and F) from hearts of 18 week-old WT (black bars) and db/db mice (white bars). State 3 and state 4 respiration rates were determined in the presence of the substrates glutamate/malate, palmitoylcarnitine and state 3 respiration examined upon addition of ADP. ETC complex I, III, and IV activities were assessed spectrophotometrically by measuring the oxidation of NADH (complex I), reduction of cytochrome $\mathrm{c}$ (complex III) and oxidation of cytochrome c (complex IV). Respiration rates are expressed as $\mathrm{nmol} / \mathrm{min} / \mathrm{mg}$ of protein while enzymatic activities are expressed as activity $/ \mathrm{min} / \mathrm{mg}$ of protein \pm SEM; $\mathrm{N}=6$ for each group. ${ }^{*} P<0.05$ for WT vs. $\mathrm{db} / \mathrm{db}$. 
Figure 4.3

A
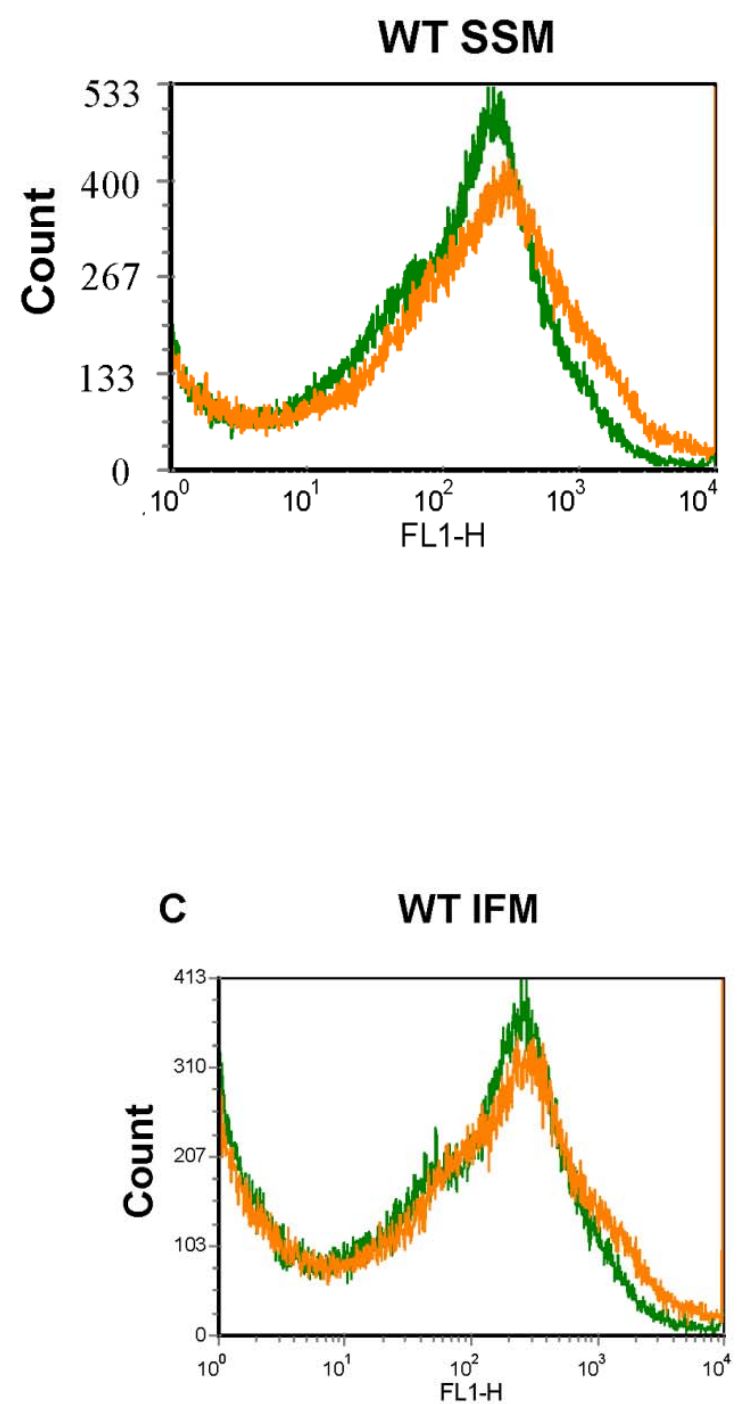

B

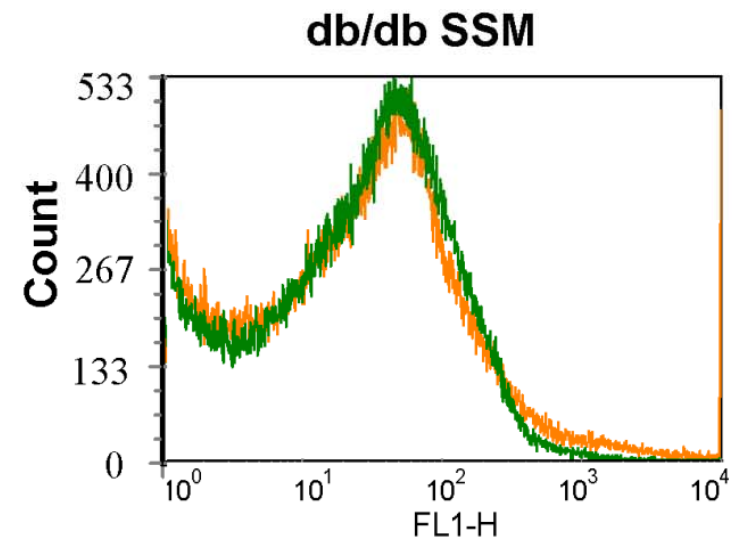

D

$\mathrm{db} / \mathrm{db}$ IFM

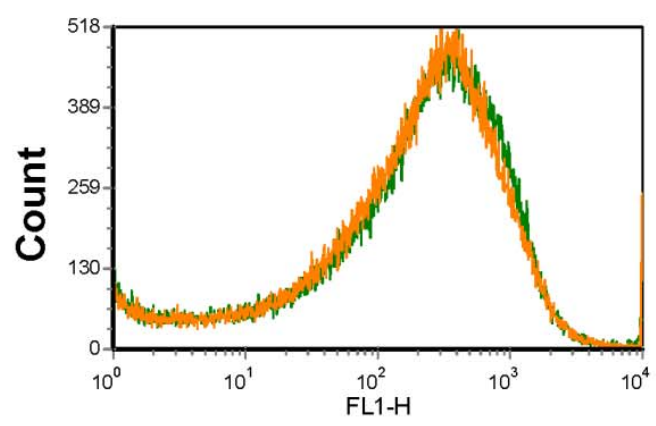


Figure 4.3

E

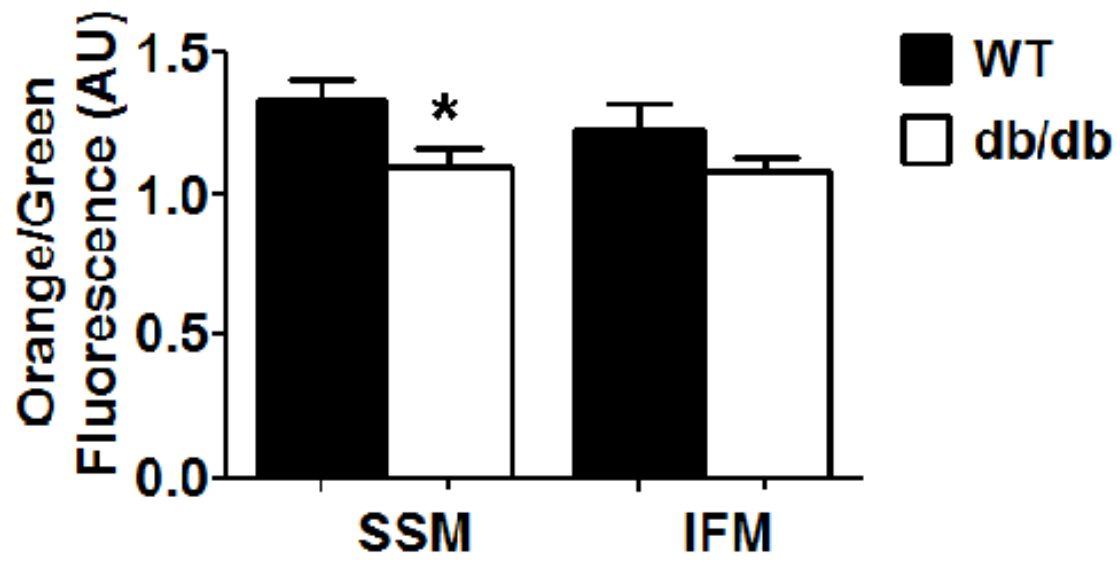


Figure 4.3. Flow cytometric analysis of mitochondrial subpopulation membrane potential $\left(\Delta \Psi_{\mathrm{m}}\right)$ in WT and $\mathrm{db} / \mathrm{db}$ heart. Membrane potential $\left(\Delta \Psi_{\mathrm{m}}\right)$ was assessed by staining mitochondrial subpopulations with JC-1 dye and assessing the shift from green to orange fluorescence with flow cytometry. Representative histograms showing green and orange fluorescence in WT SSM (A) vs. db/db SSM (B) and WT IFM (C) vs. $\mathrm{db} / \mathrm{db}$ IFM (D). $\Delta \Psi_{\mathrm{m}}$ was calculated based on orange/green fluorescence ratios in WT vs. $\mathrm{db} / \mathrm{db}$ cardiac mitochondrial subpopulations (E). Orange/green fluorescence are expressed as arbitrary units (AU) \pm SEM; $\mathrm{N}=4$ for each group. ${ }^{*} P<0.05$ for WT vs. $\mathrm{db} / \mathrm{db}$. 
Figure 4.4

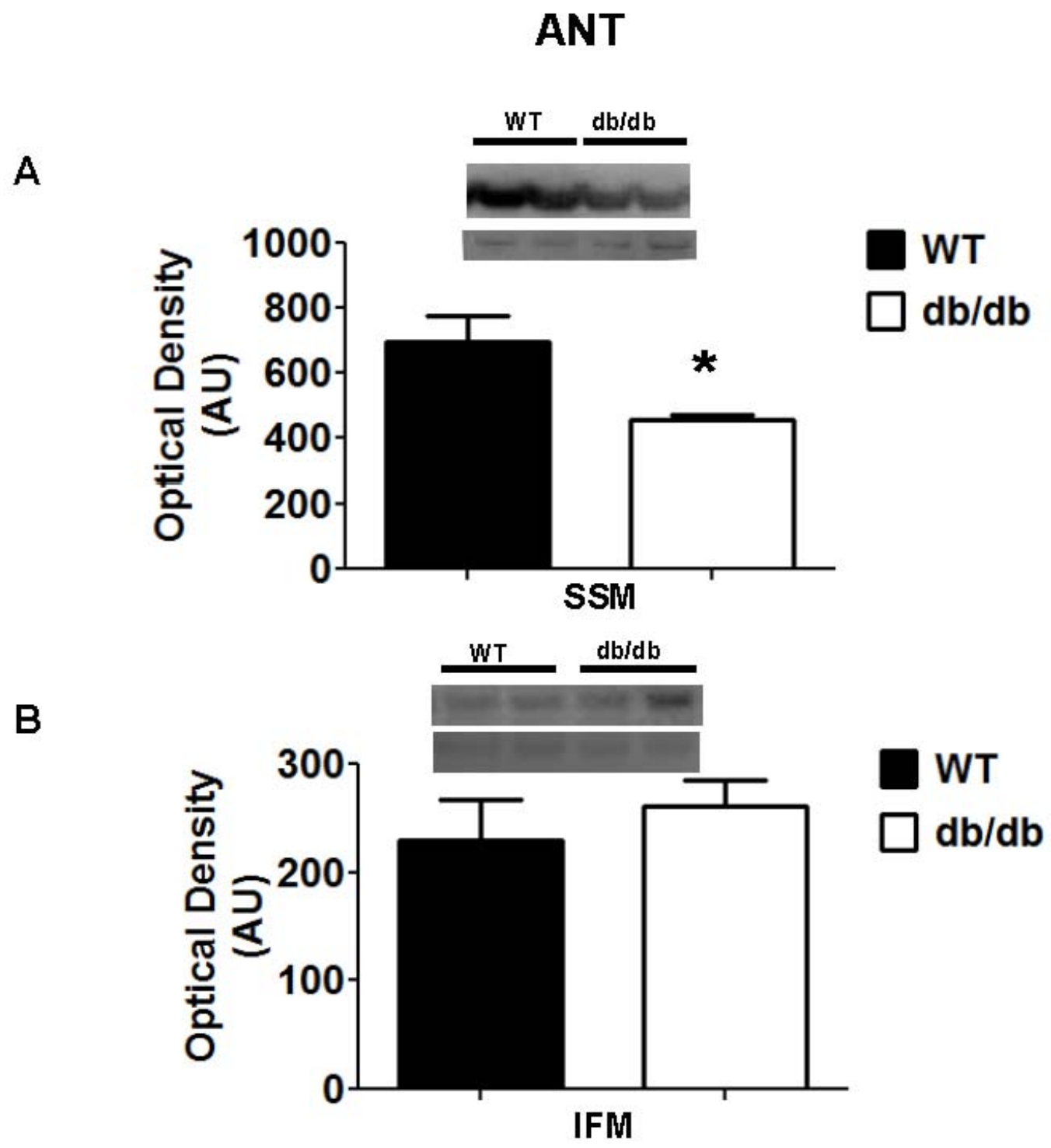


Figure 4.4. ANT protein expression in mitochondrial subpopulations from WT and $\mathbf{d b} / \mathbf{d b}$ hearts. Representative Western blots and densitometry analysis for total ANT protein in (A) SSM and (B) IFM from $\mathrm{WT}$ and $\mathrm{db} / \mathrm{db}$ hearts. Values are represented as mean $\pm \mathrm{SEM} ; \mathrm{N}=5$ for each group. ${ }^{*} P<0.05$ for WT vs. db/db. 
Figure 4.5

A

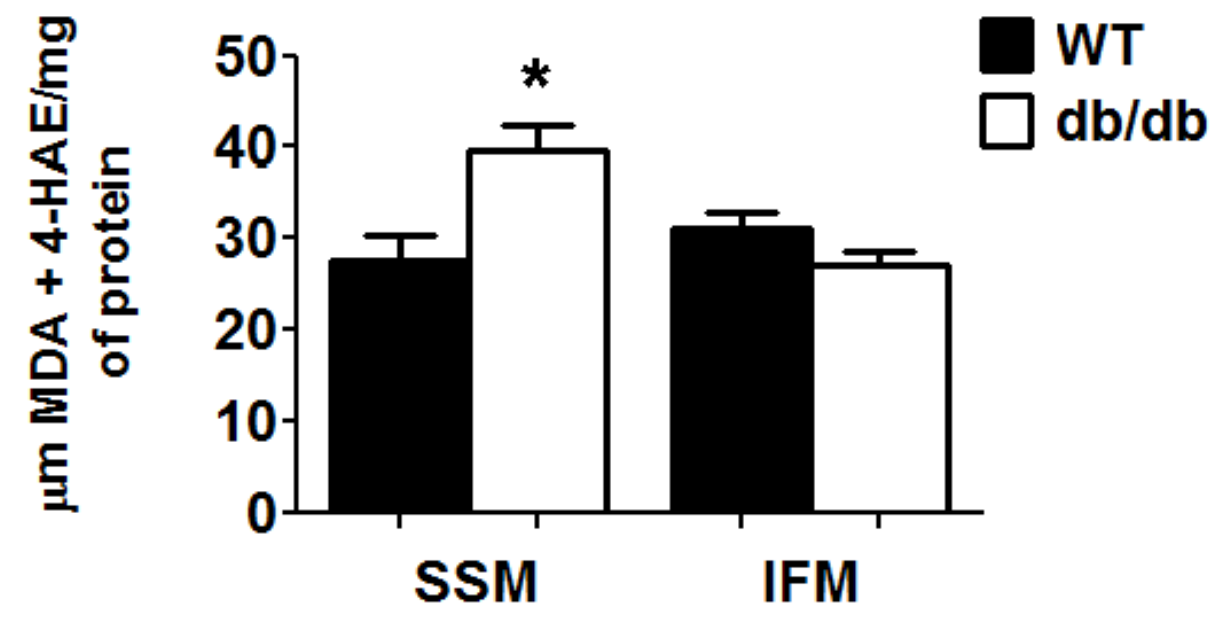


Figure 4.5

B

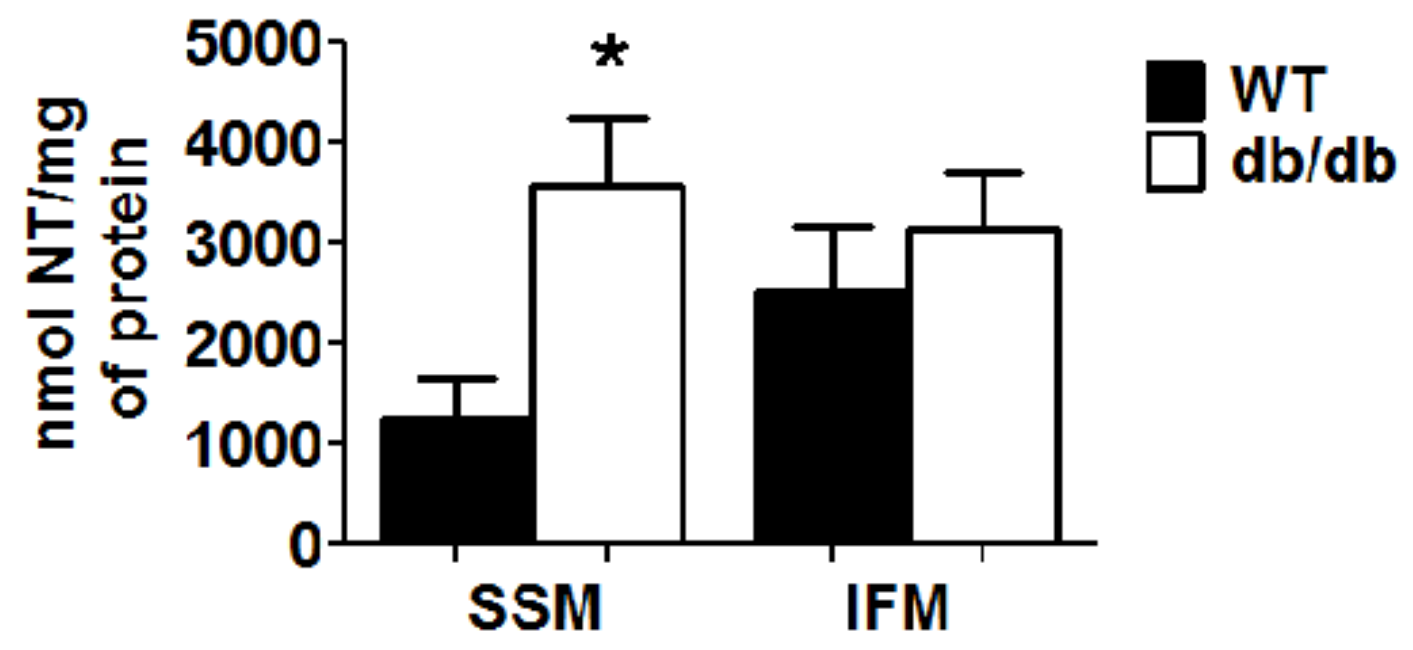


Figure 4.5. Oxidative damage in mitochondrial subpopulations from WT and $\mathbf{d b} / \mathbf{d b}$ hearts. Oxidative damage to lipids was assessed in mitochondrial subpopulations by measuring the lipid peroxidation byproducts malondialdehyde (MDA) and 4-hydroxyalkenals (4-HAE) using a colorimetric assay and compared against a standard curve of known 4-HAE and MDA concentrations. Data are expressed as $\mu \mathrm{mol}$ of MDA + 4-HAE per $\mathrm{mg}$ of mitochondrial protein. Oxidative damage to proteins was assessed by quantifying 3-nitrotyrosine residues through a sandwich ELISA and concentration determined by comparing results against a known protein nitrotyrosine standard curve. Results are expressed as nmol nitrotyrosine/mg of protein. Values for both lipid peroxidation and protein nitrosylation are expressed as mean $\pm \mathrm{SEM} ; \mathrm{N}=5$ for each group. ${ }^{*} P<0.05$ for WT vs. db/db. 
Figure 4.6

A

Cytochrome C oxidase subunit VIla1

\begin{tabular}{|c|c|c|c|c|c|c|c|c|}
\hline Sequence & Modifications & $\Delta$ Mass & Theor $\mathrm{m} / \mathbf{z}$ & Theor $\mathbf{z}$ & Spectrum & $115: 114$ & $116: 114$ & $117: 114$ \\
\hline LFQADNDLPVHLK & $\begin{array}{c}\text { iTAQ4plex@N-term; } \\
\text { iTRAQ4plex(K)@13 }\end{array}$ & -0.0345 & 1798.0101 & 1 & 1.7 .5 .64 .13 & 0.9519 & 1.3105 & 0.7626 \\
\hline
\end{tabular}

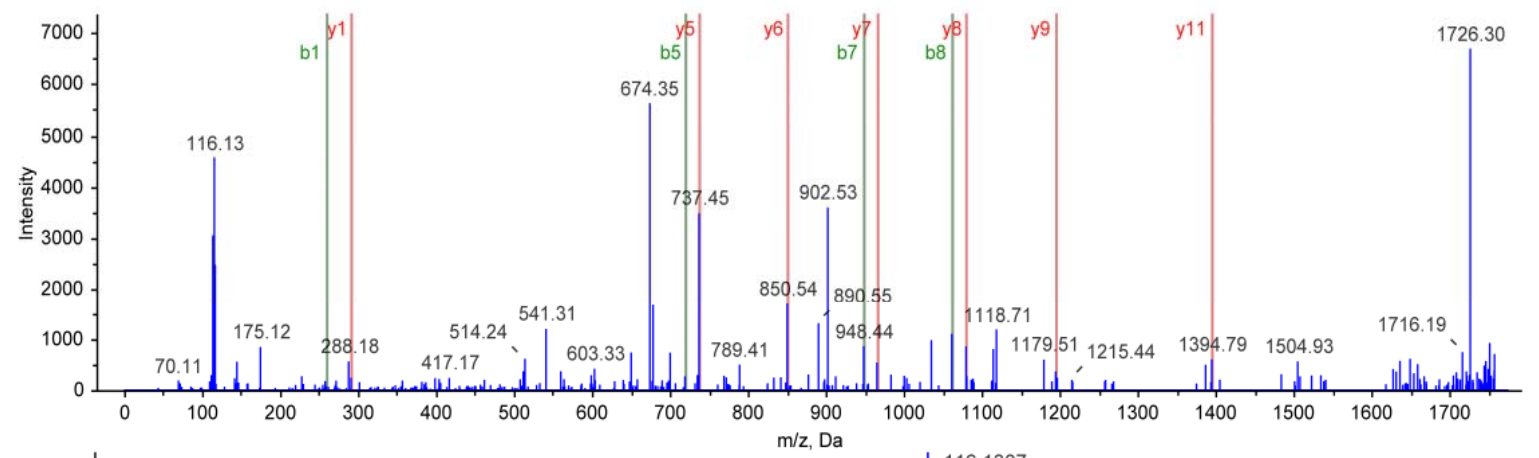

B

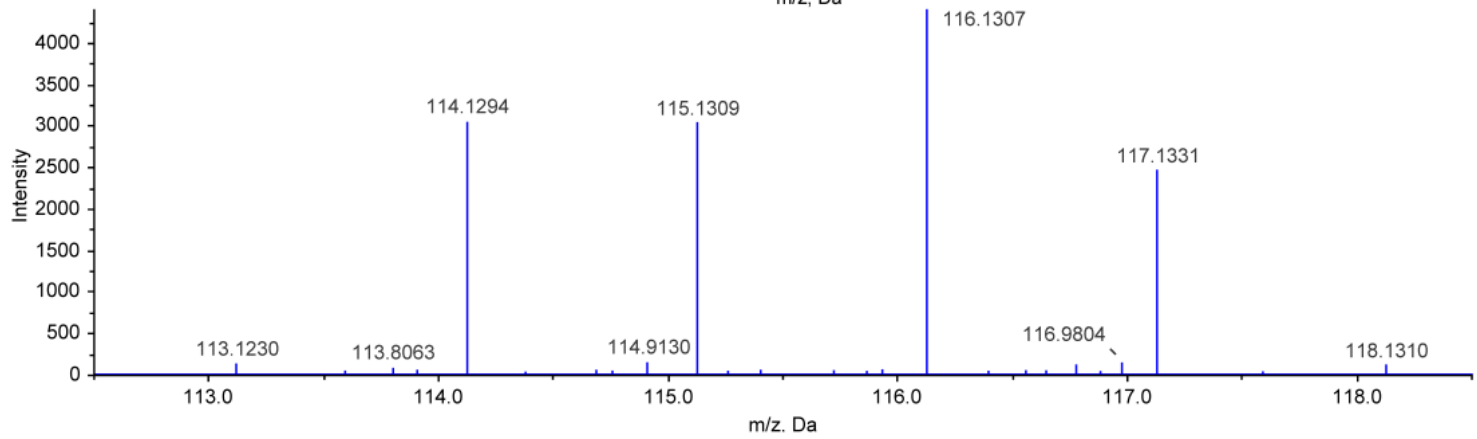


Figure 4.6

Cytochrome $\mathrm{C}$ oxidase subunit Vlla1

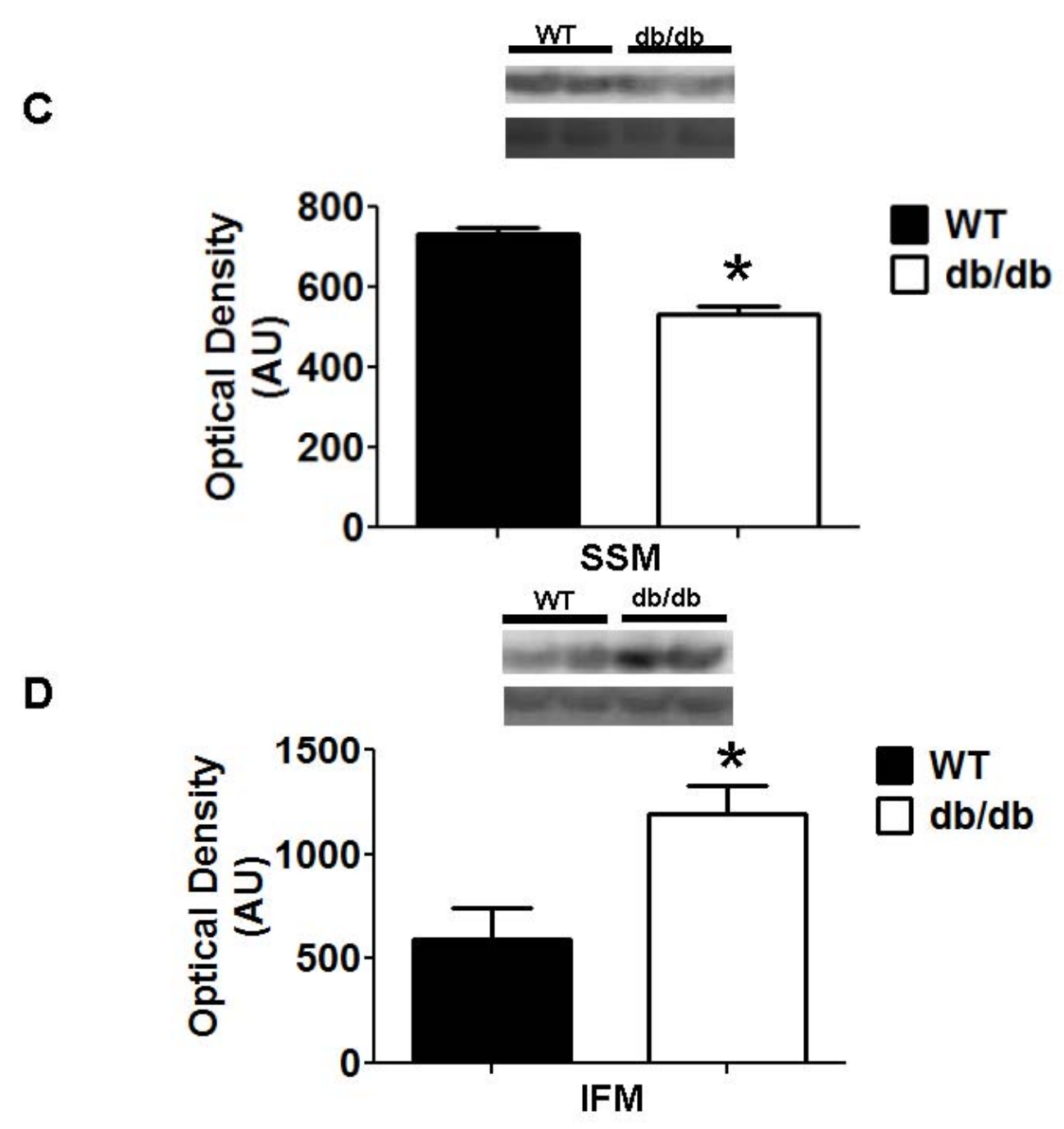


Figure 4.6. Representative ITRAQ $^{\mathrm{TM}}$ spectra and Western blot analysis. Isolated mitochondrial subpopulations from WT and db/db hearts were labeled with iTRAQ ${ }^{\mathrm{TM}}$ reagents 114 (db/db SSM), 115 (db/db IFM), 116 (WT SSM), 117 (WT IFM) and combined for analysis with mass spectrometry. (A) Representative spectra of simultaneous quantitation of cytochrome $\mathrm{c}$ oxidase subunit VIla1 peptide in WT and $\mathrm{db} / \mathrm{db}$ mitochondrial subpopulations. (B) Spectra for the reporter groups of the $\mathrm{TTRAQ}{ }^{\mathrm{TM}}$ reagents $(114,115,116$ and 117) from cytochrome $c$ oxidase subunit Vlla1 peptide MS/MS spectra are shown. These spectra were used along with other peptides to simultaneously quantify cytochrome $c$ oxidase subunit VIla1 in WT and $\mathrm{db} / \mathrm{db}$ mitochondrial subpopulations. Representative Western blot and densitometry analysis of cytochrome $\mathrm{c}$ oxidase subunit VIla1 in SSM (C) and IFM (D) from WT and db/db hearts. Values are represented as mean \pm SEM; $N=5$ for each group. ${ }^{*} P<0.05$ for $W T$ vs. $\mathrm{db} / \mathrm{db}$. 


\begin{tabular}{|c|c|c|c|}
\hline Protein Name & SSMD/SSMC & IFMD/IFMC & SSMC/IFMC \\
\hline \multicolumn{4}{|l|}{ Mitochondrial Fatty Acids Beta-oxidation } \\
\hline Hydroxyacyl - Coenzyme A dehydrogenase & 1.08 & 1.21 & 1.26 \\
\hline Glutamate oxaloacetate transaminase 2 & 1.13 & .74 & .75 \\
\hline Mitochondrial trifunctional protein, Beta subunit & 1.20 & 1.19 & NS \\
\hline Acyl - coenzyme A dehydrogenase, medium chain & 1.16 & NS & .80 \\
\hline 3-ketoacyl-CoA thiolase, mitochondrial & 1.24 & NS & NS \\
\hline Acetyl-Coenzyme A acetyltransferase 1 & NS & .30 & .54 \\
\hline Dihyrolipoamide dehydrogenase & NS & .75 & .85 \\
\hline Dodecenoyl-Coenzyme A delta isomerase & NS & 1.29 & NS \\
\hline \multicolumn{4}{|l|}{ Mitochondrial Respiratory Chain } \\
\hline $\begin{array}{l}\text { ATP synthase, } \mathrm{H}^{+} \text {transporting mitochondrial FO complex, } \\
\text { subunit d }\end{array}$ & .90 & NS & .88 \\
\hline ATP synthase $\mathrm{H}^{+}$transporting FO subunit $\mathrm{F}$ isoform 2 & .90 & 1.25 & 1.35 \\
\hline Ubquinol cytochrome c reductase core protein 2 & .76 & NS & NS \\
\hline Cytochrome b-c1 complex subunit 1 & NS & 1.44 & NS \\
\hline Cytochrome c1, heme protein, mitochondrial & .85 & NS & 1.21 \\
\hline Electron transferring flavoprotein, beta polypeptide & .88 & 1.44 & 1.79 \\
\hline Succinate dehydrogenase Fp subunit & NS & .68 & .66 \\
\hline NADH dehydogenase (ubiquinone) 1 alpha subcomplex 9 & NS & 1.16 & NS \\
\hline NADH dehydrogenase (ubiquinone) 1 beta subcomplex, 10 & .79 & NS & NS \\
\hline NADH dehydrogenase (ubiquinone) Fe-S protein 6 & .70 & NS & .79 \\
\hline NADH dehydrogenase ubiquinone FE-S protein 7 & .76 & NS & NS \\
\hline Cytochrome C oxidase, subunit VIc & .88 & 1.28 & 1.41 \\
\hline Cytochrome C oxidase subunit Va & .75 & 1.20 & 1.75 \\
\hline Cytochrome C oxidase, subunit VIIa1 & .64 & NS & .82 \\
\hline Cytochrome c oxidase subunit IV isoform 1 & NS & 1.16 & NS \\
\hline
\end{tabular}




\begin{tabular}{|c|c|c|c|}
\hline Cytochrome C oxidase, subunit VIb polypeptide 1 & NS & .81 & NS \\
\hline Cytochrome C oxidase subunit II & NS & 1.32 & NS \\
\hline \multicolumn{4}{|l|}{ TCA Cycle } \\
\hline Isocitrate dehydrogenase 2 (NADP+) & 1.12 & 1.23 & NS \\
\hline Isocitrate dehydrogenase $3\left(\mathrm{NAD}^{+}\right)$, alpha & NS & 1.28 & 1.35 \\
\hline malate dehydrogenase 2, NAD & NS & .79 & NS \\
\hline $\begin{array}{l}\text { Succinyl-CoA ligase [ADP-forming] subunit beta, } \\
\text { mitochondrial }\end{array}$ & NS & 1.30 & \\
\hline \multicolumn{4}{|l|}{ Amino Acid Metabolism } \\
\hline Dihydrolipamide s-succinyltransferase & NS & .61 & NS \\
\hline \multicolumn{4}{|l|}{ Oxidative Stress Related } \\
\hline Peroxiredoxin V & .80 & NS & NS \\
\hline \multicolumn{4}{|l|}{ Transport Proteins } \\
\hline $\begin{array}{l}\text { carrier family } 25 \text { (mitochondrial carrier, phosphate carrier), } \\
\text { member } 3\end{array}$ & 1.15 & .87 & 1.17 \\
\hline Grp75, mitochondrial hsp70 & .80 & NS & 1.26 \\
\hline $\begin{array}{l}\text { Solute carrier family } 25 \text { (mitochondrial carnitine/acylcarnitine } \\
\text { translocase), member } 20\end{array}$ & NS & 1.28 & .64 \\
\hline VDAC 2 & 1.24 & NS & .67 \\
\hline Carnitine O-palmitoyltransferase 1, muscle isoform & NS & 1.37 & .647 \\
\hline Voltage-dependent anion-selective channel 1 & NS & .70 & .77 \\
\hline Voltage-dependent anion-selective channel 3 & NS & .50 & NS \\
\hline \multicolumn{4}{|l|}{ Miscellaneous } \\
\hline NipSnap homolog 2 & NS & 1.46 & 1.36 \\
\hline Es 1 protein & 1.15 & .67 & .85 \\
\hline Elongation factor tu, mitochondrial & NS & .46 & .73 \\
\hline Aldehyde dehydrogenase & .69 & NS & NS \\
\hline Mirq-like protein & NS & 1.21 & \\
\hline Desmin & .66 & NS & NS \\
\hline
\end{tabular}


Table 4.1. Proteomic analysis of mitochondrial subpopulations from WT and $\mathrm{db} / \mathrm{db}$ hearts. ITRAQ ${ }^{\mathrm{TM}}$ analysis of proteins identified and significantly changing, categorized into groups consisting of fatty acid oxidation (FAO), citric acid cycle (TCA), mitochondrial respiratory chain, amino acid related, oxidative stress, transport and miscellaneous proteins in isolated SSM and IFM from WT and db/db hearts. Cells highlighted in red represent decreased protein expression in the $\mathrm{db} / \mathrm{db}$ mitochondria compared to WT, while cells highlighted in green are increased in $\mathrm{db} / \mathrm{db}$ compared to WT. All values presented indicate a significant difference of at least ${ }^{*} P<0.05$ for $W T$ vs. $d b / d b$, while NS represents no significant differences between any group. $\mathrm{N}=4$ for each group. 


\section{SUPPLEMENTAL MATERIALS AND METHODS}

\section{Blood Plasma Analyses}

Blood samples, taken from mice immediately after sacrifice, were centrifuged and the supernatant frozen at $-80^{\circ} \mathrm{C}$ for determination of free fatty acid (FFA) concentrations and insulin levels. FFAs were measured using a spectrophotometric based free fatty acid concentration kit (Biovision, Mountain View, CA), and insulin levels were determined using an enzyme-linked immunosorbent assay kit (Alpco Immunoassays, Salem NH).

\section{Citrate Synthase Activity}

Citrate synthase activity, a marker of mitochondrial matrix enzyme activity, was measured using a diode array spectrophotometer at $412 \mathrm{~nm}$ as previously described (3).

\section{Fasting Glucose Levels}

Mice were fasted 4-6 hours and blood glucose levels were determined using an Ascencia ${ }^{\circledR}$ Contour ${ }^{\mathrm{TM}}$ glucometer (Bayer Healthcare, Mishawaka, IN).

\section{Cardiac contractile function}

Hearts from $\mathrm{db} / \mathrm{db}$ and WT animals were isolated and transferred to a Langendorff setup for contractile experiments as previously described $(1,2,4)$. In brief, 
hearts were removed from anesthetized mice and immersed in cold cardioplegic solution. After cannulation of the aorta on a luer stub, hearts were perfused retrograde at $37^{\circ} \mathrm{C}$ with a modified Krebs-Henseleit buffer [containing (in mmol/l) 0.5 pyruvate, 0.4 caprylic acid, $118 \mathrm{NaCl}, 4.7 \mathrm{KCl}, 2.25 \mathrm{CaCl}_{2}, 1.2 \mathrm{MgSO}_{4}, 1.2 \mathrm{KH}_{2} \mathrm{PO}_{4}, 25 \mathrm{NaHCO}_{3}, 0.5$ $\mathrm{Na}_{2}$ EDTA, and 5.5 glucose] that was subjected to oxygenation. A small fluid-filled balloon was inserted into the left ventricular (LV) cavity and coupled to a pressure transducer (Argon Medical Devices, Athens, TX). The balloon was inflated until the enddiastolic pressure reached $10 \mathrm{mmHg}$. Platinum wires were placed on the surface of the right atrium and used to pace the heart at 420 beats/min $(7 \mathrm{~Hz})$ with increasing voltage until the heart rate was consistent (captured). During this time period, hearts remained submerged in a $37^{\circ} \mathrm{C}$ jacketed chamber, and a heat lamp attached to a variable alternating current transformer was used to maintain temperature, which was monitored by a needle temperature probe inserted just before the luer cannula (Physitemp, Clifton, $\mathrm{NJ}$ ). Hearts were perfused for $15 \mathrm{~min}$ to achieve stable cardiac function and then followed for an additional $45 \mathrm{~min}$, yielding a total protocol time of $1 \mathrm{~h}$. During this time, digitized recordings of the LV pressure and its first derivative as well as heart rate were captured on an IBM-compatible PC using ChartPro software (AD Instruments, Colorado Springs, CO). LV peak systolic pressure, developed pressure (DP), maximum speed of contraction $(+d P / d t)$, and maximum speed of relaxation $(-d P / d t)$ were analyzed from the recordings using ChartPro software. 


\section{SUPPLEMENTAL REFERENCES}

1. Hollander JM, Lin KM, Scott BT, and Dillmann WH. Overexpression of PHGPX and HSP60/10 protects against ischemia/reoxygenation injury. Free Radic Biol Med 35: 742-751, 2003.

2. Hollander JM, Martin JL, Belke DD, Scott BT, Swanson E, Krishnamoorthy V, and Dillmann WH. Overexpression of wild-type heat shock protein 27 and a nonphosphorylatable heat shock protein 27 mutant protects against ischemia/reperfusion injury in a transgenic mouse model. Circulation 110: 3544-3552, 2004.

3. Matsuoka Y, and Srere PA. Kinetic studies of citrate synthase from rat kidney and rat brain. The Journal of biological chemistry 248: 8022-8030, 1973.

4. Trost SU, Belke DD, Bluhm WF, Meyer M, Swanson E, and Dillmann WH. Overexpression of the sarcoplasmic reticulum $\mathrm{Ca}(2+)$-ATPase improves myocardial contractility in diabetic cardiomyopathy. Diabetes 51: 1166-1171, 2002. 
Figure 4.7

\section{UCP3}

A

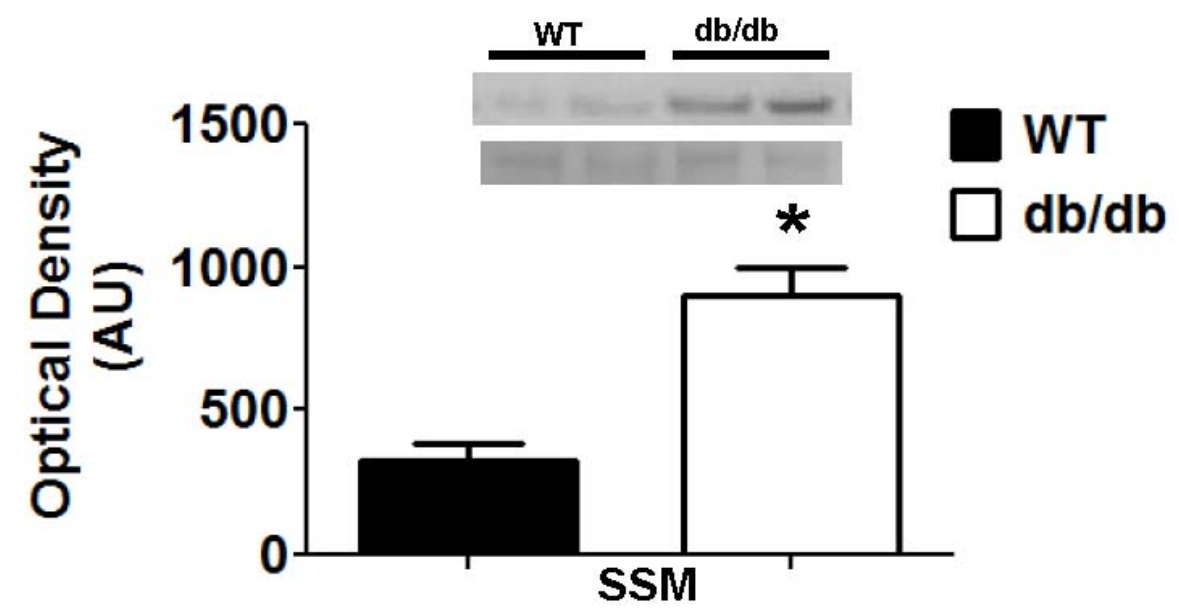

B

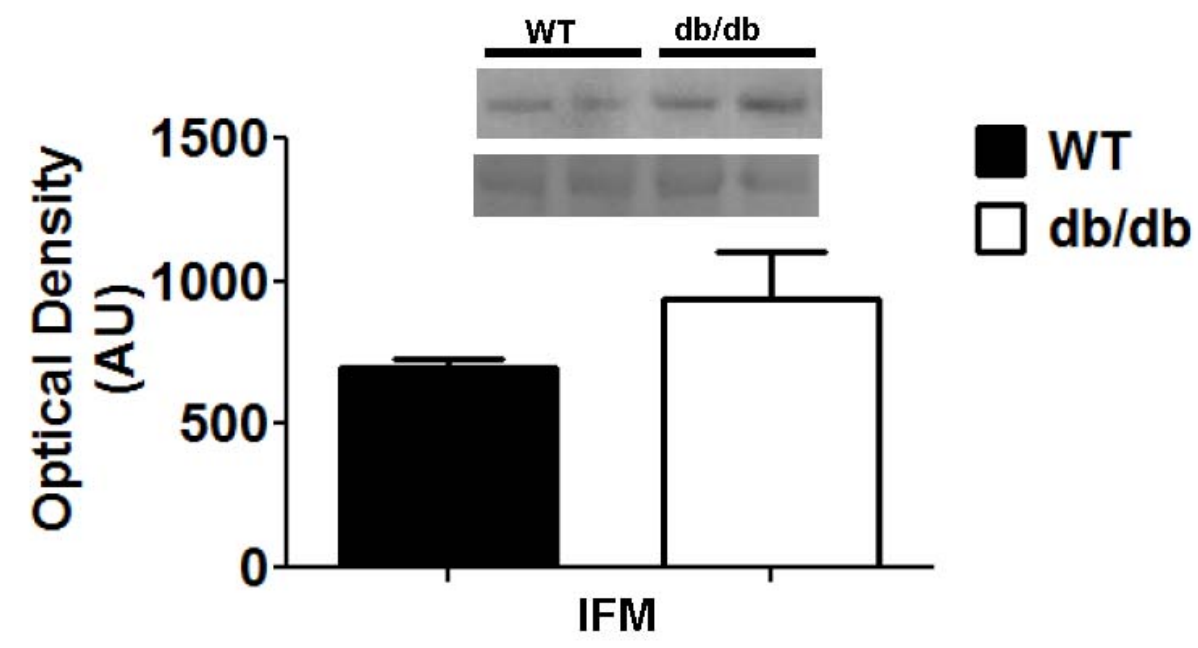


Figure 4.7. UCP3 protein expression in mitochondrial subpopulations from WT and $\mathbf{d b} / \mathbf{d b}$ hearts. Representative Western blots and densitometry analysis for total UCP3 protein in (A) SSM and (B) IFM from $\mathrm{WT}$ and $\mathrm{db} / \mathrm{db}$ hearts. Values are represented as mean $\pm \mathrm{SEM} ; \mathrm{N}=5$ for each group. ${ }^{*} P<0.05$ for WT vs. $\mathrm{db} / \mathrm{db}$. 
Figure 4.8

A Ubquinol cytochrome $\mathrm{c}$ reductase core protein 2
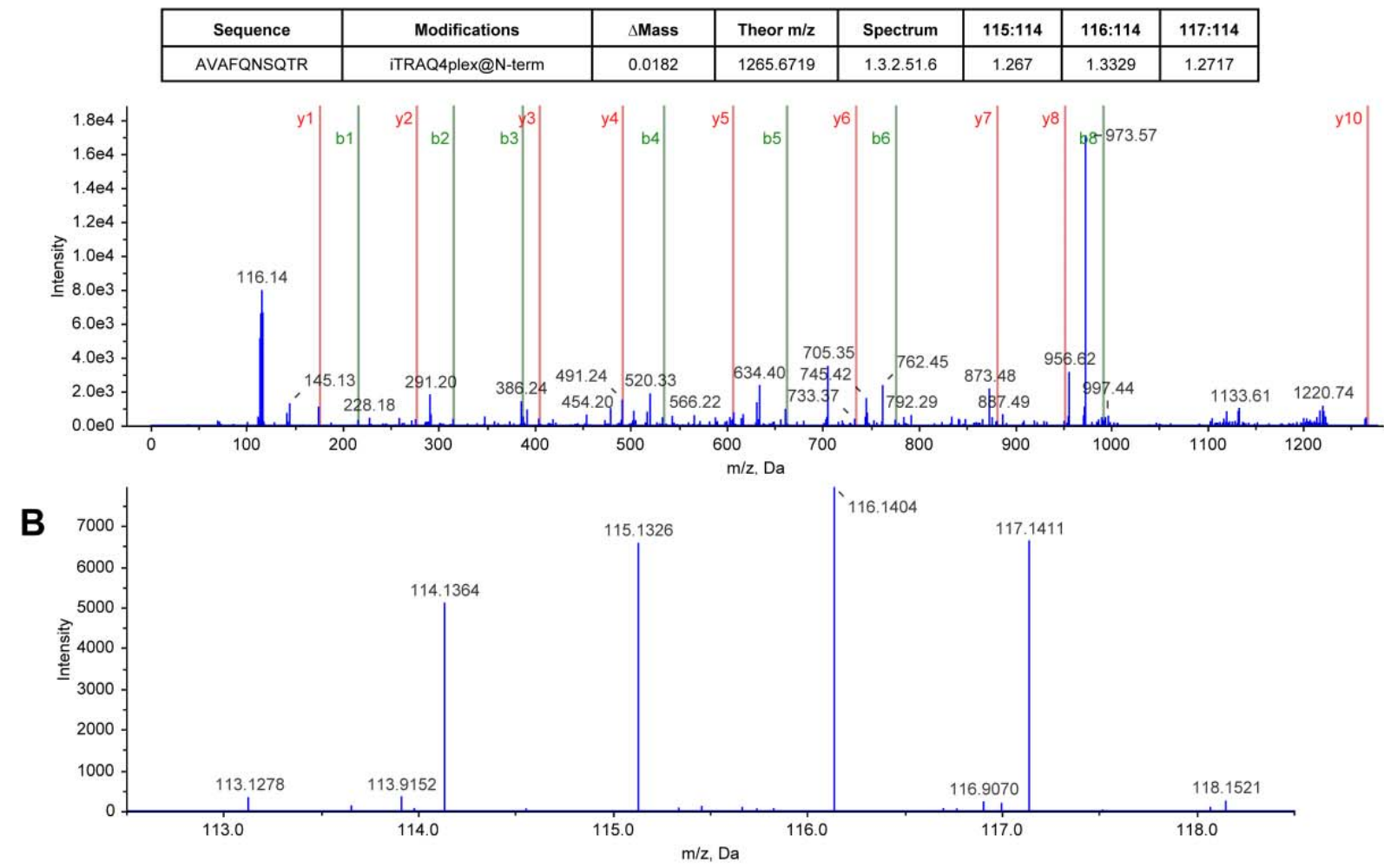
Figure 4.8

C

Ubquinol cytochrome c reductase core protein 2

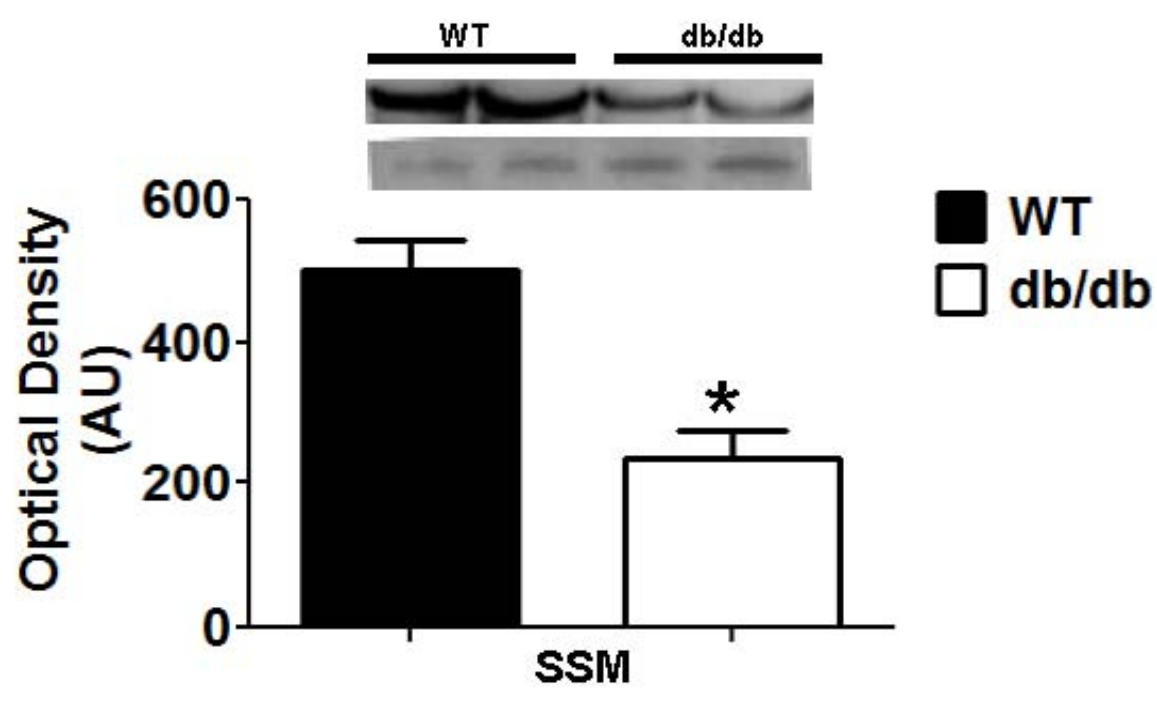

D

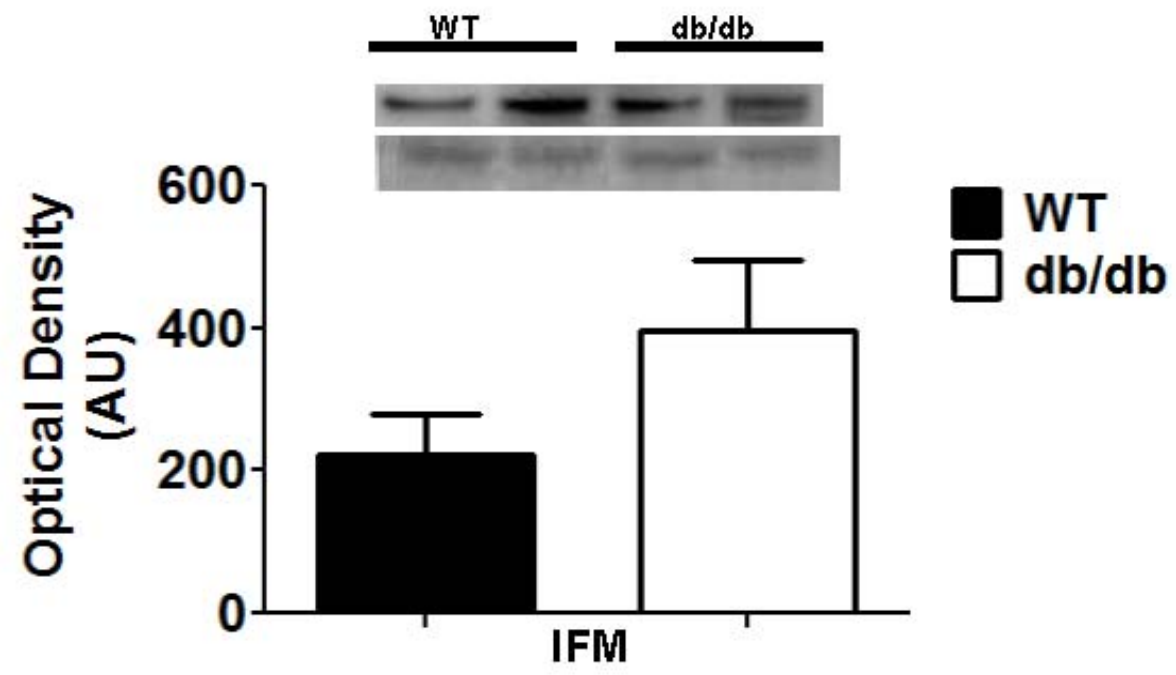


Figure 4.8. Representative ITRAQ $^{\mathrm{TM}}$ spectra and Western blot analysis. Isolated mitochondrial subpopulations from WT and db/db hearts were labeled with iTRAQ ${ }^{\mathrm{TM}}$ reagents 114 (db/db SSM), 115 (db/db IFM), 116 (WT SSM), 117 (WT IFM) and combined for analysis with mass spectrometry. (A) Representative spectra of simultaneous quantitation of a ubiquinol cytochrome $\mathrm{c}$ reductase core protein 2 peptide in WT and $\mathrm{db} / \mathrm{db}$ mitochondrial subpopulations. (B) Spectra for the reporter groups of the iTRAQ $^{\text {TM }}$ reagents $(114,115,116$ and 117) from ubiquinol cytochrome $c$ reductase core protein 2 peptide MS/MS spectra are shown. These spectra were used along with other peptides to simultaneously quantify ubiquinol cytochrome c reductase core protein 2 in WT and $\mathrm{db} / \mathrm{db}$ mitochondrial subpopulations. Representative Western blot and densitometry analysis of ubiquinol cytochrome c reductase core protein 2 in SSM (C) and IFM (D) from $\mathrm{WT}$ and $\mathrm{db} / \mathrm{db}$ hearts. Values are represented as mean $\pm \mathrm{SEM} ; \mathrm{N}=5$ for each group. ${ }^{*} P<0.05$ for WT vs. db/db. 


\section{Table 4.2}

\begin{tabular}{|l|l|l|}
\hline Metabolic Parameters & \multicolumn{1}{|c|}{ WT } & \multicolumn{1}{|c|}{ Db/Db } \\
\hline Body Weight (g) & $28.8 \pm .77$ & $54.4 \pm 1.0^{*}$ \\
\hline Plasma Glucose (mg/dL) & $127.7 \pm 6$ & $543 \pm 27^{*}$ \\
\hline Plasma Insulin (ng/mL) & $.82 \pm .19$ & $2.16 \pm .22^{*}$ \\
\hline Plasma Free Fatty Acids (uM) & $230.8 \pm 36.9$ & $668.3 \pm 97.7^{*}$ \\
\hline
\end{tabular}


Table 4.2. Metabolic Parameters. Assessment of metabolic parameters in $\mathrm{WT}$ and $\mathrm{db} / \mathrm{db}$ hearts, including body weight $(\mathrm{g})$, blood glucose $(\mathrm{mg} / \mathrm{dL})$, plasma insulin $(\mathrm{ng} / \mathrm{mL})$ and plasma free fatty acids (FFA; $\mu \mathrm{mol})$. Values are expressed as means \pm SEM; $N=7$ for each group. ${ }^{*} P<0.05$ for WT vs. $\mathrm{db} / \mathrm{db}$ mice. 


\section{Table 4.3}

\begin{tabular}{|l|l|l|}
\hline Contractile Parameters & \multicolumn{1}{|c|}{ WT } & \multicolumn{1}{|c|}{ Db/Db } \\
\hline DP $(\mathrm{mm} / \mathrm{Hg})$ & $138 \pm 4.8$ & $57.5 \pm 10.1^{*}$ \\
\hline -DP/DT (mm/Hg/s) & $5932 \pm 454.3$ & $2072 \pm 308.9^{*}$ \\
\hline$+\mathrm{DP} / \mathrm{DT}(\mathrm{mm} / \mathrm{Hg} / \mathrm{s})$ & $3665 \pm 144.1$ & $1321 \pm 132.2^{*}$ \\
\hline
\end{tabular}




\section{Table 4.3. Cardiac contractile function from $W T$ and $\mathrm{db} / \mathrm{db}$ hearts.}

Functional measurements were performed in Langendorff-perfused hearts and are expressed as the rate of contraction $(+d P / d t)$, rate of relaxation ($\mathrm{dP} / \mathrm{d} t$ ), and developed pressure (DP). Values are expressed as means \pm SEM; $N=5$ for each group. ${ }^{*} P<0.05$ for $W T$ vs. $\mathrm{db} / \mathrm{db}$ mice. 


\section{Table 4.4}

\begin{tabular}{|l|l|l|}
\hline Mitochondrial Marker Enzyme & \multicolumn{1}{|c|}{ WT } & \multicolumn{1}{|c|}{ Db/Db } \\
\hline Citrate synthase, um/mg protein & & \\
\hline SSM & $4367 \pm 476$ & $4394 \pm 562$ \\
\hline IFM & $4195 \pm 191$ & $4870 \pm 455$ \\
\hline Mitochondrial yield (mg/g wt) & & \\
\hline SSM & $9.7 \pm .308$ & $9.1 \pm .219$ \\
\hline IFM & $6.84 \pm .15$ & $7.7 \pm .17$ \\
\hline
\end{tabular}


Table 4.4. Mitochondrial marker enzyme and protein yield. Citrate synthase activities from isolated SSM and IFM. Mitochondrial protein yields in isolated SSM and IFM. Values are expressed as means \pm SEM; $\mathrm{N}=5$ for $W T$ and $\mathrm{db} / \mathrm{db}$ mice. 
Table 4.5

\begin{tabular}{|c|c|c|}
\hline Protein Diabetic SSM & $\begin{array}{l}\text { Peptide Sequence } \\
\text { Diabetic SSM }\end{array}$ & $\begin{array}{l}\text { Post Translational Modification } \\
\text { Diabetic SSM }\end{array}$ \\
\hline $\begin{array}{l}\text { ATP synthase, } \mathrm{H}+\text { transporting, } \\
\text { mitochondrial } \mathrm{F} 1 \text { complex, alpha subunit }\end{array}$ & QKTGTAEMSSILEER & GIn->pyro-Glu@N-term \\
\hline $\begin{array}{l}\text { ATP synthase, } \mathrm{H}+\text { transporting, } \\
\text { mitochondrial } \mathrm{F} 1 \text { complex, alpha subunit }\end{array}$ & DSRLVLEVAQHLGESTVR & Dehydrated(D)@1; Deamidated(R)@3 \\
\hline aconitase 2, & AVTQEFGPVPDTAR & Decanoyl(T)@3 \\
\hline 3-ketoacyl-CoA thiolase & RTPFGAYGGLLKDFSATDLTEFAAR & Deamidated(R)@1; Dehydrated(T)@2 \\
\hline $\begin{array}{l}\text { electron transferring flavoprotein, beta } \\
\text { polypeptide [Mus musculus]; unnamed } \\
\text { protein product [Mus musculus] }\end{array}$ & YATLPNIMKAK & Trimethyl(K)@9 \\
\hline $\begin{array}{l}\text { cytochrome c oxidase, subunit VIc [Mus } \\
\text { musculus] }\end{array}$ & SSGALLPKPQMR & Protein Terminal Acetyl@N-term \\
\hline $\begin{array}{l}\text { cytochrome c oxidase, subunit VIc [Mus } \\
\text { musculus] }\end{array}$ & PNCGTHYKLVPHQMAH & $\begin{array}{l}\text { Carbamyl@N-term; Deamidated(N)@2; } \\
\text { Carbamidomethyl(C)@3 }\end{array}$ \\
\hline $\begin{array}{l}\text { malate dehydrogenase 2, NAD } \\
\text { (mitochondrial) [Mus musculus]; malate } \\
\text { dehydrogenase [Mus musculus] }\end{array}$ & GYLGPEQLPDCLKGCDVVVIPAGVPR & $\begin{array}{l}\text { Oxidation(P)@5; Oxidation(P)@9; } \\
\text { Carbamidomethyl(C)@11; } \\
\text { Didehydroretinylidene(K)@13; } \\
\text { Carbamidomethyl(C)@15 }\end{array}$ \\
\hline $\begin{array}{l}\text { pyruvate dehydrogenase E1 alpha } 1 \\
\text { [Mus musculus] }\end{array}$ & VLAGSAQKPASR & Deamidated(Q)@7 \\
\hline $\begin{array}{l}\text { Ndufb4 protein [Mus musculus]; NADH } \\
\text { dehydrogenase } 1 \text { beta subcomplex } 4 \\
\text { [Mus musculus] }\end{array}$ & $\begin{array}{l}\text { SGSKYKPAPLATLPSTLDPAEYDVSPE } \\
\text { TR }\end{array}$ & Protein Terminal Acetyl@N-term \\
\hline cytochrome c-1 [Mus musculus] & AANNGALPPDLSYIVR & Deamidated(N)@4 \\
\hline cytochrome c-1 [Mus musculus] & ANNGALPPDLSYIVR & Carbamyl@N-term; Oxidation(N)@3 \\
\hline $\begin{array}{l}\text { ubiquinol-cytochrome c reductase, } \\
\text { complex III subunit VII, isoform CRA_b } \\
\text { [Mus musculus] }\end{array}$ & GREFGNLAR & Protein Terminal Acetyl@N-term \\
\hline
\end{tabular}




\begin{tabular}{|c|c|c|}
\hline $\begin{array}{l}\text { NADH dehydrogenase (ubiquinone) Fe-S } \\
\text { protein } 1 \text { [Mus musculus] }\end{array}$ & EDWKIIR & Oxidation(W)@3 \\
\hline isocitrate dehydrogenase $3(\mathrm{NAD}+)$ alpha & IAEFAFEYARNNHR & Deamidated(N)@11 \\
\hline glutamate oxaloacetate transaminase 2 & MNLGVGAYRDDNGKPYVLPSVR & Deamidated(N)@12 \\
\hline glutamate oxaloacetate transaminase 2 & KMNLGVGAYRDDNGKPYVLPSVR & Deamidated(N)@13 \\
\hline glutamate oxaloacetate transaminase 2 & ILIRPLYSNPPLNGAR & Deamidated(N)@13 \\
\hline $\begin{array}{l}\text { hypothetical protein LOC380773 [Mus } \\
\text { musculus]; RIKEN cDNA } 1810035 \mathrm{~L} 17 \text {, } \\
\text { isoform CRA_c [Mus musculus]; SRA } \\
\text { stem-loop-interacting RNA-binding } \\
\text { protein, }\end{array}$ & AASAIKGLSALR & Protein Terminal Acetyl@N-term \\
\hline $\begin{array}{l}\text { low density lipoprotein receptor-related } \\
\text { protein } 4 \text { [Mus musculus] }\end{array}$ & TCSPGMNSFLIFAR & $\begin{array}{l}\text { Carbamidomethyl(C)@2; } \\
\text { Oxidation(M)@6 }\end{array}$ \\
\hline $\begin{array}{l}\text { ATP synthase, } \mathrm{H}+\text { transporting, } \\
\text { mitochondrial F1F0 complex, subunit e } \\
\text { [Mus musculus] }\end{array}$ & VPPVQVSPLIKFGR & Oxidation(P)@8 \\
\hline $\begin{array}{l}\text { succinate dehydrogenase complex, } \\
\text { subunit B, iron sulfur (Ip }\end{array}$ & AATVGVSLKRGFPAAVLGR & Oxidation(P)@13 \\
\hline Protein IFM Diabetic & Peptide Sequence Diabetic IFM & $\begin{array}{l}\text { Post Translational Modification } \\
\text { Diabetic IFM }\end{array}$ \\
\hline $\begin{array}{l}\text { 3-ketoacyl-Coenzyme A thiolase } \\
\text { (trifunctional protein), alpha subunit }\end{array}$ & AGLEQGSDAGYLAESQK & $\begin{array}{l}\text { Carbamidomethyl@N-term; } \\
\text { Dehydrated(E)@4; Deamidated(Q)@5 }\end{array}$ \\
\hline $\begin{array}{l}\text { 3-ketoacyl-Coenzyme A thiolase } \\
\text { (trifunctional protein), alpha subunit }\end{array}$ & SPKPVVAAISGSCLGGGLELAIACQ & $\begin{array}{l}\text { HexNAc(S)@12; } \\
\text { Carbamidomethyl(C)@13; } \\
\text { Carbamidomethyl(C)@24 }\end{array}$ \\
\hline $\begin{array}{l}\text { cytochrome c oxidase, subunit VIc [Mus } \\
\text { musculus] }\end{array}$ & SSGALLPKPQMR & Protein Terminal Acetyl@N-term \\
\hline mitochondrial malate dehydrogenase 2 & AKVAVLGASGGIGQPLSLLLKNSPLVSR & $\begin{array}{l}\text { Lys->Allysine }(\mathrm{K}) @ 21 \\
\text { Deamidated(N)@22 }\end{array}$ \\
\hline
\end{tabular}




\begin{tabular}{|c|c|c|}
\hline $\begin{array}{l}\text { NADH dehydrogenase (ubiquinone) Fe-S } \\
\text { protein } 5\end{array}$ & EGKYTPPPHHSGREEPRP & Methyl(E)@1; Delta:H(2)C(2)(K)@3 \\
\hline coenzyme Q9 homolog [Mus musculus] & LNQVLEEEQKLVQLGQAEKR & Deamidated(Q)@3 \\
\hline $\begin{array}{l}\text { ubiquinol cytochrome } \mathrm{c} \text { reductase core } \\
\text { protein } 2\end{array}$ & AGSRYEDSNNLGTSHLLR & $\begin{array}{l}\text { Carbamidomethyl@N-term; } \\
\text { Dehydrated(S)@3; Deamidated(R)@4 }\end{array}$ \\
\hline 2,4-dienoyl CoA reductase 1 & SLAAEWGR & Dioxidation(W)@6 \\
\hline enoyl Coenzyme A hydratase & NSSVGLIQLNRPK & Deamidated(N)@1 \\
\hline dihydrolipoamide S-succinyltransferas & NDVITVQTPAFAESVTEGDVRWEK & Deamidated(N)@1 \\
\hline glutamate oxaloacetate transaminase 2 & KMNLGVGAYRDDNGKPYVLPSVR & Deamidated(N)@13 \\
\hline
\end{tabular}


Table 4.5. Post-translational modifications in mitochondrial subpopulations from WT and $\mathrm{db} / \mathrm{db}$ hearts. Multidimensional protein identification technology (MudPIT) was used to identify post-translational modifications of proteins in SSM and IFM from WT and db/db mitochondria. Peptides presented represent only those peptides that have posttranslational modifications in the $\mathrm{db} / \mathrm{db}$ SSM (A) and IFM (B) and not present in the WT group. 


\section{Chapter 5:}

\section{Mitochondria-Specific Transgenic Overexpression of Phospholipid Hydroperoxide Glutathione Peroxidase (GPX4) Attenuates Ischemia/Reperfusion Associated Cardiac Dysfunction}

As published in Free Radical Biol Med. 2008 Sep 15; 45(6):855-65.

Erinne R. Dabkowski, Courtney L. Williamson, and John M. Hollander

West Virginia University School of Medicine, Division of Exercise Physiology, and Center for Interdisciplinary Research in Cardiovascular Sciences, Morgantown, WV 26506

Running Title: GPx4 and ischemia/reperfusion injury 


\begin{abstract}
Ischemia/reperfusion (I/R) injury elicits damage to mitochondria. Antioxidants provide protection from I/R-induced mitochondrial damage. The goal of this study was to determine the impact of mitochondria-specific overexpression of GPx4 (PHGPx) on cardiac function following I/R. Transgenic mice were created in which PHGPx was overexpressed solely in the mitochondrion (mPHGPx). MPHGPx and littermate control hearts were subjected to global no-flow ischemia $(20 \mathrm{~min})$ followed by reflow reperfusion (30, 60, $90 \mathrm{~min}$ ). Following I/R, mPHGPx hearts possessed significantly better rates of contraction, developed pressures, and peak-systolic pressures as compared to controls $(P<0.05)$. No differences were observed in rates of relaxation or end-diastolic pressures. Lipid peroxidation was significantly lower in mitochondria from $\mathrm{mPHGPx}$ hearts as compared to controls, following $\mathrm{I} / \mathrm{R}(P<0.05)$. Electron transport chain (ETC) complex I, III, and IV activities were significantly higher in MPHGPx hearts as compared to controls, following I/R $(P<0.05)$. MPHGPx overexpression enhanced ETC complex I, III, and IV activities in subsarcolemmal mitochondria (SSM; $P<0.05)$, and ETC complex I and III activities in interfibrillar mitochondria (IFM; $P<0.05$ ) following I/R. These results indicate that mitochondria-specific GPx4 overexpression protects cardiac contractile function and preserves ETC complex activities following I/R. These results provide further rationale for the use of $\mathrm{mPHGPx}$ as a therapeutic protectant.
\end{abstract}

Ischemia, Reperfusion, Mitochondria, Free radical scavenger 


\section{INTRODUCTION}

Oxidative processes play a major role in the pathogenesis of myocardial ischemia/reperfusion $(I / R)$ injury. Central to the pathogenesis is the role played by reactive oxygen species (ROS). ROS generated during I/R initiate and potentiate pathological, biochemical and physiological responses that include membrane lipid peroxidation (LPO), disruption of cytoskeletal structure, disturbance of cellular oxidoreductive status, enzyme inactivation, deterioration of mitochondrial function, and impairment of excitation contraction coupling [1-6]. Though many potential sources of ROS exist in myocardium, the mitochondrion is considered to be the primary site [7-9]. A number of studies have observed an increase in ROS production from heart mitochondria after I/R $[10,11]$. Several sites in the electron transport chain (ETC) are particularly prone to ROS formation and include oxidizable electron carriers in the inner mitochondrial membrane [7]. This has implications for ETC proteins because a major constituent of many of these structures is iron-sulphur centers [12], which can react with ROS and produce highly reactive species such as the hydroxyl radical $\left(\mathrm{OH}^{\cdot}\right)$ [13]. Mitochondrial ROS are generated during I/R due to electron leakage from the respiratory chain. These electrons react with residual molecular oxygen leading to superoxide $\left(\mathrm{O}_{2} \cdot\right)$ generation [14]. A large burst of ROS occurring within the first minute of reperfusion contributes to the phenomenon of reperfusion injury $[2,11,15]$. Oxidoreductive changes, decreased high energy phosphate compounds, and increased oxidative stress indirectly indicate that ROS are increased during $I / R[2,16]$.

A primary determinant of the extent of $I / R$ injury is antioxidant defense capacity, which is modest in the myocardium relative to its highly aerobic nature [17, 18]. ROS produced in the mitochondria are regulated by mitochondrial antioxidant enzymes which include manganese superoxide dismutase (MnSOD), glutathione peroxidase (GPx1), and mitochondrial phospholipid hydroperoxide glutathione peroxidase (mPHGPx; GPx4) $[19,20]$. GPx4 is a monomeric enzyme with two distinct forms, a long form (L-form), which contains a mitochondrial targeting sequence and exists in the mitochondrion (mPHGPx), and a short form (S-form), void of a mitochondrial targeting sequence which 
exists outside of the mitochondrion [21]. A third GPx4 form was identified in rat sperm nuclei, which contains a nuclear targeting signal, however the role of this GPx4 isoform is not entirely clear [21]. MPHGPx is localized predominantly at contact sites between the outer and inner mitochondrial membrane [22], and is of particular interest because it is the only antioxidant enzyme capable of reducing peroxidized acyl groups in phospholipids [23], fatty acid hydroperoxides [24, 25], and cholesterol peroxides [26], in biological membranes. Since mPHGPx is a lipophilic enzyme associated with subcellular membranes, it can act directly on lipid hydroperoxides formed in the mitochondria [26], and as a result, is the primary antioxidant defense against oxidation of biomembranes within the mitochondrion.

Highly reactive products derived from peroxidized lipids, such as malondialdehyde (MDA) and 4-hydroxy-2-nonenal (4-HNE), may contribute to the deleterious effects associated with $I / R[27,28]$. These reactive products can disrupt ETC function by damaging complex constituents, which facilitates necrotic and apoptotic cell death [29]. Maintenance of mitochondrial phospholipid bilayer integrity is crucial for preservation of mitochondrial protein components, and appropriate membrane fluidity all of which influence mitochondrial function. MPHGPX overexpression in stable cell lines inhibits the apoptotic program by preventing mitochondrial cytochrome $C$ release, which may be associated with attenuation of oxidative stress and preservation of the phospholipid, cardiolipin [19, 30]. Our previous studies have indicated that adenoviral vector-mediated mPHGPx overexpression can preserve mitochondrial function, decrease necrosis, and inhibit apoptosis in neonatal cardiac myocytes subjected to simulated I/R (hypoxia/reoxygenation), all of which are associated with an attenuation of oxidative stress [31]. Homozygous null GPx4deficient mice die in utero and cell lines derived from GPx4-deficient heterozygous mice are more susceptible to oxidative insult [32]. Transgenic mice overexpressing GPx4 have been shown to be protective against oxidative stress-induced apoptosis, however these animals express both the mitochondrial (L-form) and nonmitochondrial (S-form) forms of the enzyme making it difficult to determine the sole contribution of the mitochondrial form (L-form) during pathological insult [33]. A recent study indicates that 
GPx4 heterozygous knockout mice possess increased life span, and decreased fatal lymphoma incidence, complicating our understanding of the role played by this protein [34]. Interestingly, Mclung et al. reported increased incidence of insulin resistance and obesity in GPx1 transgenic mice. These authors speculate that persistent overexpression of GPx1 in the cytosol may interfere with cytosolic ROS $\left(\mathrm{H}_{2} \mathrm{O}_{2}\right.$ tone) required for essential signal transduction pathways that influence insulin receptor $\beta$ subunit and Akt phosphorylation. As a result, maintenance of normal protein-tyrosine phosphatase regulation in the insulin signaling cascade is disrupted, and phosphorylation of insulin receptor attenuated, leading to insulin resistance and subsequent obesity [35].

In light of these reports, we generated a novel transgenic mouse that expresses only the mitochondrial form (L-form; mPHGPx) of GPx4. This model was designed to eliminate the influence of enhanced cytosolic peroxide scavenging, and enable determination of the specific role played by GPx4 in the mitochondrion. Since the mitochondrion is central to the pathogenesis of myocardial I/R injury, we sought to determine the impact of mPHGPx overexpression on cardiac contraction and ETC function following global myocardial $I / R$ insult. Our results indicate that mitochondriaspecific overexpression of mPHGPx provides protection to both contractile and mitochondrial function following $\mathrm{l} / \mathrm{R}$ insult. 


\section{MATERIALS AND METHODS}

\section{MPHGPX Transgenic Mouse Development}

The animal models used in this study conform to the NIH guidelines for the care and use of laboratory animals and were approved by the West Virginia University, School of Medicine Animal Care and Use Program. MPHGPx transgenic mouse lines were generated using a chimeric transgene consisting of the rat PHGPx (GPx4) gene inserted into the vector PCAGGS as previously described [36-38]. The mPHGPx gene, a kind gift from Dr. Donna Driscoll of the Cleveland Clinic Foundation (Cleveland, $\mathrm{OH}$, USA), consists of a 5' mitochondria targeting sequence (MT), the GPX4 CDNA, and 3'UTR which includes a selenocysteine insertion sequence (SCI) (Figure 1A) [39]. Briefly, the pCAGGS vector places the mPHGPx gene under the control of the human cytomegalovirus (CMV) immediate early enhancer and chicken $\beta$-actin (Ch. $\beta$-actin) promoter with first intron (Figure 1B) [40]. The mPHGPX cDNA was inserted into the Xho I cloning site of pCAGGS via blunt-end ligation, as a Bam $\mathrm{HI} /$ Not I fragment of approximately $870 \mathrm{bp}$ (Figure 1B). The chimeric transgene was cut out of the plasmid by Ssp I and Bam HI digestion, purified, and used to generate the transgenic mice. The construct was given to the West Virginia University Transgenic Animal Core Facility where the pronucleus of fertilized eggs from superovulated FVB female mice crossed with FVB male mice were injected with 1-2 pl of the purified DNA fragment at a concentration of $2 \mu \mathrm{g} / \mathrm{ml}$, and transferred into the oviduct of pseudopregnant CD-1 mice. All control and transgenic mice were generated using an FVB background, and experimental procedures were performed on animals of approximately 4-7 months of age. Animals were maintained in individual microisolator cages within the Transgenic Barrier Facility and given food and water ad libitum. 


\section{MPHGPX Transgenic Mouse Screening}

Mouse litters were delivered after 19-20 days of gestation. To verify if the chimeric transgene was present in the genome, DNA from 4 week old mice was isolated from tail clips using a Qiagen DNeasy tissue kit (Qiagen, Valencia, CA). Transgene screening was performed using a real-time PCR (RT-PCR) approach, in which we probed for the CMV immediate early enhancer (CMVIE) region using a custom designed fluorometric probe (Applied Biosystems, Foster City, CA). Using this approach, only DNA that contains the CMVIE viral sequence is detected. Briefly, sequence for the CMV region (Figure 1B) was submitted to Applied Biosystems Custom TaqMan ${ }^{\circledR}$ Gene Expression Assays Service and a custom FAM $^{\mathrm{TM}}$ dye-labeled TaqMan® Minor Groove Binder (MGB) probe and two unlabeled PCR primers were created, by qualifying the target sequence using publicly available bioinformatics databases. Isolated tail DNA, probe, primers, and universal master mix were brought up to $25 \mu \mathrm{l}$ and RT-PCR performed in a 96 well plate using an Applied Biosystems 7900HT Fast Real-Time PCR system (Applied Biosystems, Foster City, CA). All samples were run in duplicate. Mouse tail DNA from known positive transgenic mice generated using the same pCAGGS vector [37], as well as known littermate negative mice were included as internal positive and negative controls, respectively. A reaction time versus cycle number amplification plot was generated and transgene positive and negative animals determined. An RT-PCR approach for screening transgenic animals was chosen because of its enhanced sensitivity, assay speed, decreased potential for a false positive result, and high throughput potential.

\section{Cardiac Contractile Function}

Hearts from mPHGPx and littermate control animals were isolated and transferred to a Langendorff set-up for contractile studies as previously described [37, 41]. In brief, hearts were removed from anesthetized mice, and immersed in cold cardioplegic solution. After cannulation of the aorta on a luer stub, hearts are perfused 
(80 $\mathrm{mm} \mathrm{Hg}$ constant pressure) retrograde at $37^{\circ} \mathrm{C}$ with a modified Krebs-Henseleit buffer (in mmol/L: $118 \mathrm{NaCl}, 4.7 \mathrm{KCl}, 2.25 \mathrm{CaCl}_{2}, 1.2 \mathrm{MgSO}_{4}, 1.2 \mathrm{KH}_{2} \mathrm{PO}_{4}, 25 \mathrm{NaHCO}_{3}$, $0.5 \mathrm{Na}_{2}$ EDTA, 5.5 glucose) that was subject to oxygenation. A small fluid-filled balloon was inserted into the left ventricular (LV) cavity and coupled to a pressure transducer (Argon Medical Devices, Athens, TX). The balloon was inflated until the end-diastolic pressure (EDP) reached $10 \mathrm{mmHg}$. Platinum wires are placed on the surface of the right atrium and used to pace the heart at 420 beats/min $(7 \mathrm{~Hz})$ with increasing voltage until the heart rate was consistent (captured). During this time period, hearts remain submerged in a $37^{\circ} \mathrm{C}$ jacketed chamber, and a heat lamp attached to a variable AC transformer was utilized to maintain temperature which was monitored by a needle temperature probe inserted just prior to the luer cannula (Physitemp, Clifton, NJ). Hearts were perfused for 20 minutes to achieve a stable cardiac function, and then placed through an ischemic period in which pacing and perfusion were ceased for 20 minutes. During this time period, hearts remained submerged in a $37^{\circ} \mathrm{C}$ jacketed chamber to maintain temperature. Following the no-flow ischemic period, pacing (420 beats $/ \mathrm{min} ; 7 \mathrm{~Hz}$ ) and perfusion were reinitiated and continued for 90 minutes. Digitized recordings of the ventricular pressure and its first derivative, as well as heart rate were captured on an IBM-compatible PC using ChartPro software (AD Instruments, Colorado Springs, CO). Left ventricular developed pressure (DP), maximum speed of contraction $(+d P / d t)$, and maximum speed of relaxation (-dP/dt), peak-systolic pressure (PSP), and EDP were analyzed from the recordings using ChartPro software.

\section{Creatine Kinase Release}

Creatine Kinase $(\mathrm{CK})$ release was used as a determinant of cellular disruption and damage as previously described [37]. CK activities were measured spectrophotometrically at $340 \mathrm{~nm}$ using a kit (Teco Diagnostics, Anaheim, CA) where release was determined as the activity in the perfusates at various times prior to (Pre) 
and during the reperfusion period $(1,2,3,4,5,6,10,30,60,90 \mathrm{~min}$, respectively). CK activities were expressed as the change in absorbance, per min, per gram of tissue.

\section{Isolation of Cellular Fractions}

Isolation of cellular fractions was accomplished using a mitochondria/cytosol fractionation kit and nuclear/cytosol fractionation kit as previously described [31] (BioVision, Mountain View, CA). Isolated mitochondria fractions were suspended in sucrose buffer (in mmol/L: 220 Sucrose, 70 mannitol, 10 Tris HCL, 1 EDTA; pH 7.4) and functional measurements were performed on fresh mitochondria.

\section{Isolation of Individual Mitochondrial Subpopulations}

Hearts were rinsed in phosphate buffered saline (PBS, pH 7.4), then blotted dry and weighed. Subsarcolemmal mitochondria (SSM) and interfibrillar mitochondria (IFM) were isolated on ice following the methods of Palmer et al. [42] with minor modifications. Briefly, the ventricles were minced and homogenized 1:10 (w/v) in cold Chappell-Perry buffer (in mmol/L: $100 \mathrm{KCl}, 40$ Tris-HCl, 10 Tris-Base, $5 \mathrm{MgCl}_{2}, 1$ EDTA; pH 7.4) [42]. The homogenates were than centrifuged at $800 \times g$ for 10 minutes. The supernatant was extracted and centrifuged again at $9,000 \times g$ to isolate the SSM. The SSM pellet was washed and centrifuged two more times at 9,000 $\times \mathrm{g}$. The remaining pellet from the $800 \times g$ spin was resuspended in Buffer 2 (in mmol/L: $100 \mathrm{KCL}, 5 \mathrm{MgSO}_{4}, 5 \mathrm{EGTA}$, $50 \mathrm{Tris}-\mathrm{HCl} ; \mathrm{pH} \mathrm{7.4)}$ and exposed to $5 \mathrm{mg} / \mathrm{kg}$ of trypsin for 10 minutes [43]. After 10 minutes, the IFM pellet was diluted two-fold with buffer and centrifuged at 5,000 $\times g$ for 5 minutes. The supernatant was discarded and the pellet was resuspended in buffer and centrifuged at $800 \times g$ for 10 minutes. The supernatant was saved and the pellet resuspended and centrifuged again at $800 \times g$ for 10 minutes to maximize IFM yield. 
Next, supernatants were combined and centrifuged at 9,000 $\times g$ to yield the IFM. IFM were washed several times and centrifuged at 9,000 $\times g$ for 10 minutes. Pellets were resuspended in sucrose buffer as described previously and protein concentrations were determined using the Bradford method and bovine serum albumin as a standard [44].

To verify the mitochondria subpopulation isolation procedures, we performed flow cytometric analyses using a FACS Calibur equipped with a $15 \mathrm{mw} 488 \mathrm{~nm}$ argon laser and 633 red diode laser (Becton and Dickinson, San Jose, CA, USA). MitoTracker Deep Red 633 (Invitrogen, Carlsbad, CA) was used to selectively stain intact mitochondria (EM 633nm) and exclude debris, which enabled accurate gating (R1) of the mitochondria. Gated events (20,000 per sample) were examined using the forward scatter (FSC) and side scatter (SSC) detectors, and represented in FSC vs. SSC density plots. Geometric mean (arbitrary units, AU) representing FSC (logarithmic scale) was used as an indicator of size while values from SSC (logarithmic scale) were used to indicate complexity in the subpopulations. Though the FSC arbitrary unit is proportional to the individual mitochondria particle size, the absolute value still remains an arbitrary unit. Thus, to confirm the absolute mitochondria size, we utilized a flow cytometry size calibration kit (Invitrogen, Carlsbad, CA) which contains a set of microsphere suspensions $(0.5 \mu \mathrm{M}-6 \mu \mathrm{M})$ to serve as reliable size references for flow cytometric analyses. All flow cytometric measurements were performed under the supervision of the West Virginia University Flow Cytometry Core Facility.

\section{MPHGPX Protein Analysis}

SDS polyacrylamide gel electrophoresis (SDS-PAGE) was run on $4-12 \%$ gradient gels as described [45], with equal amounts of protein loaded. Relative amounts of mPHGPx, Oct-1, cyclophilin D, Grp75, and GAPDH proteins were determined using specific antibodies; anti-GPx4 rabbit antibody (Product \# 10005258; 
Cayman Chemical, Ann Arbor, MI), anti-Oct-1 rabbit antibody (Product\# sc-232; Santa Cruz Biotech, Santa Cruz, CA), anti-cyclophilin D rabbit antibody (Product\# PA1-028; Affinity Bioreagents, Golden, CO), anti-Grp75 mouse antibody (Product\# SPS825; Stressgen, Victoria, BC, Canada), and anti-GAPDH mouse antibody (Product\# sc32233; Santa Cruz Biotech, Santa Cruz, CA). The secondary antibody for all rabbit primary antibodies was a goat anti-rabbit IgG HRP conjugate (Product \# 10004301; Cayman Chemical, Ann Arbor, Ml). The secondary antibody for all mouse primary antibodies was a goat anti-mouse IgG HRP conjugate (Product \# 31430; Pierce, Rockford, IL). Detection of signal was performed according to the manufacturer's instructions, using Pierce ECL Western Blotting Substrate (Pierce, Rockford, IL). Autoradiographic signals were assessed using a G:Box Bioimaging System (Syngene, Frederick, MD), and the data captured and analyzed using GeneSnap/GeneTools software (Syngene, Frederick, MD).

\section{MPHGPx Enzymatic Activity Analysis}

Phosphatidylcholine hydroperoxide $(\mathrm{PCOOH})$ was used as a substrate for PHGPx activity measurements, and the $\mathrm{PCOOH}$ was synthesized using the method of Maiorino et al. [46], with modifications [47]. Once the reaction mixture was generated, it was passed through a Sep-Pak $\mathrm{C}_{18}$ cartridge (Waters, Milford MA) that had been washed previously and equilibrated with water. The final substrate was eluted with $2 \mathrm{ml}$ of methanol and utilized as a substrate for PHGPx activity assessment. PHGPx activity was measured as previously described [46].

\section{Antioxidant Enzyme Protein Analysis}

SDS-PAGE was run on $4-12 \%$ gradient gels as above [45], with equal amounts of protein loaded. Relative amounts of MnSOD, copper-zinc superoxide dismutase (CuZnSOD), GPx1, and catalase proteins were determined using specific antibodies; 
anti-MnSOD goat antibody (Product \# A300-448A; Bethyl Laboratories, Montgomery, TX), anti-CuZnSOD rabbit antibody (Product \#2779; Cell Signaling, Danvers, MA), antiGPx1 rabbit antibody (Product \# ab16798; Abcam, Cambridge, MA), and anti-catalase rabbit antibody (Abcam, Cambridge, MA). The secondary antibodies were a goat antirabbit IgG HRP conjugate (Product \# 10004301; Cayman Chemical, Ann Arbor, MI) and a rabbit anti-goat IgG HRP conjugate (Product \# 31402; Pierce, Rockford, IL), respectively. Detection of autoradiographic signal was performed as above.

\section{Lipid Peroxidation Products}

Peroxidation of lipids was assessed by measurement of malondialdehyde (MDA) and 4-hydroxy-2(E)-nonenal (4-HNE), stable end products formed from the oxidation of polyunsaturated fatty acids and esters. Equal volumes of freshly isolated mitochondria were analyzed for MDA and 4-HNE using a colorimetric assay kit (Oxford Biomedical Research Company, Oxford, MI). This assay is based on the reaction of a chromogenic reagent, N-methyl-2-phenylindole with MDA and 4-HNE at $45{ }^{\circ} \mathrm{C}$. One molecule of either MDA or 4-HNE reacts with two molecules of the reagent to yield a stable chromophore with maximal absorbance at $586 \mathrm{~nm}$. Absorbance was measured on a Biotek Synergy HT plate reader (Biotek, Winooski, VT), and protein content was assessed by the Bradford method, as above, with final values expressed per mg of protein.

\section{Electron Transport Chain Complex Activities}

Respiratory capacity was assessed in total isolated mitochondria fractions and individual mitochondria subpopulations following the method of Hofhaus et al. [48] with slight modifications [31]. Following total mitochondrial or subpopulation isolation, samples were resuspended in respiration buffer (in mmol/L: $145 \mathrm{KCl}, 30 \mathrm{HEPES}, 5$ $\mathrm{KH}_{2} \mathrm{PO}_{4}, 3 \mathrm{MgCl}_{2}, 0.1 \mathrm{EGTA}$; and 1\% Fatty Acid Free BSA) and equal volumes were 
loaded into a Gilson chamber (Gilson, Middleton, WI) attached to a YSI 5300 biological oxygen monitor (YSI Inc. Yellow Springs, OH). Activities of complex I, complex III, and complex IV were determined by measuring the rate of oxygen consumed in the presence of specific substrates. These substrates were glutamate/ malate for complex I, succinate for complex III, ascorbate/N, N, N', N'-tetramethyl-p-phenylenediamine (TMPD) for complex IV and was calculated as the fraction that was sensitive to the specific inhibitors rotenone (complex I), antimycin (complex III), and sodium cyanide (complex IV). Protein content was determined following the Bradford method as above, and values expressed as nanomoles of $\mathrm{O}_{2}$ consumed per minute, per mg protein.

\section{Statistics}

Means and standard errors were calculated for all data sets. Data were analyzed with a two-way analysis of variance (ANOVA) method to evaluate the main treatment effects, mPHGPx transgene presence and I/R (Systat; version 5.03, Evanston, IL). Fisher's Least Significant Difference (LSD) post hoc tests were performed to determine the significant differences among means. When appropriate a Student's T-test was employed. $P<0.05$ was considered significant. 


\section{RESULTS}

\section{MPHGPX Transgenic Mouse Characterization}

MPHGPx transgenic mouse lines were created using a chimeric construct consisting of the mPHGPx cDNA, mitochondrial targeting sequence, and 3-UTR containing the $\mathrm{SCl}$ sequence, all of which was inserted into the multiple cloning site of the vector pCAGGS (Figure 5.1). The pCAGGS vector is under the control of the CMV promoter, and we utilized this design to screen the transgenic mouse lines (Figure 5.1). Two mPHGPx transgenic mouse lines were created, mPHGPx Line 1 and mPHGPx Line 2 (Table 5.1). DNA from mPHGPx Line 1 displayed CT values of approximately 24 , while $\mathrm{mPHGPx}$ Line 2 displayed CT value of approximately 26, indicating that Line 1 possesses slightly greater mPHGPx cDNA content (Table 5.1). Inclusion of DNA from a known positive hsp27 transgenic mouse line generated using the same pCAGGS vector [37] is included for comparison purpose, and displayed a CT value of approximately 25 (Table 5.1). In contrast, DNA from transgenic negative mice of all lines displayed CT values of approximately 34-35 which is similar to the CT value of water (Table 5.1). As a result of our findings, we chose to perform all experimentation using mPHGPx Line 1 transgenic and littermate control mice.

We determined whether transgenic mice possessed increases in mPHGPx protein levels. Total mitochondria fractions were isolated from cardiac tissue, and mPHGPx protein expression determined by Western blot analysis. MPHGPx transgenic positive mice possessed significantly higher levels of mPHGPx protein in the mitochondrial fraction of the heart (approximately 14 fold greater), as compared to littermate controls (Figure 5.2A; $P<7.8 \mathrm{E}^{-08}$ ). No significant differences were observed in Grp75 (mthsp70) which served as a mitochondria loading control (Figure 5.2A). To confirm whether the increased protein expression observed was specific for the mitochondrial fraction, we probed the mitochondria, cytosol, nuclear, and total homogenate for mPHGPx expression. Large increases in mPHGPx protein were observed in the mitochondrial fraction (Figure 5.2B). In contrast, no mPHGPx protein 
was observed in the nuclear fraction, and a modest amount of mPHGPx protein was observed in the total homogenate, which contains proteins from the mitochondria, as well as other compartments within the cell, thus diluting the signal (Figure 5.2B). We observed a trace amount of mPHGPx protein in the cytosolic fraction (Figure 5.2B) which may be a function of mPHGPx preprocessing prior to mitochondrial entry. This observation is similar to our previous findings using adenoviral vector-mediated transgene delivery of mPHGPx protein into neonatal cardiac myocytes [31]. To confirm the purity of the subcellular fractionation procedures, we probed cytosolic, mitochondria, and nuclear fractions with antibodies specific for each fraction. Our data indicate that for the most part, each fraction was relatively pure, with little or no contamination (Figure 5.2C). We further assessed the distribution of mPHGPx protein in individual mitochondrial subpopulations, and observed similar expression patterns in both SSM and IFM fractions (Figure 5.2D). To confirm that transgenic positive mice expressed functional $\mathrm{mPHGPx}$ protein, we examined phosphatidylcholine hydroperoxide $(\mathrm{PCOOH})$ scavenging. $\mathrm{PCOOH}$ is a specific substrate of GPx4. MPHGPx overexpression led to a significant $(P<0.01)$ increase of approximately 14 fold in $\mathrm{PCOOH}$ scavenging (in $\mu \mathrm{mol}$ $\mathrm{PCOOH} / \mathrm{min} / \mathrm{mg}$ protein: Control, $0.29 \pm 0.02 ; \mathrm{mPHGPx}, 4.11 \pm 0.65)$, indicating that the enhanced protein expression observed was enzymatically functional. MPHGPX transgenic overexpression had no significant impact on the protein levels of four of the major antioxidant enzymes (MnSOD, CuZnSOD, GPx1, catalase) (Figure 5.3).

\section{Cardiac Contractile Function Following Global Myocardial I/R}

Heart weight (in mg: Control, 157 \pm 8 ; mPHGPx, 156 \pm 10 ), body weight (in g: Control, 34.5 \pm 3.2 ; $\mathrm{mPHGPx}, 33.4 \pm 3.2$ ), and heart weight/body weight ratios (in $\mathrm{mg} / \mathrm{g}$ : Control, $4.7 \pm 0.3$; mPHGPx, 4.9 \pm 0.3 ) were not significantly altered by $\mathrm{mPHGPx}$ transgene expression. To determine whether mPHGPX overexpression influenced cardiac contractile function, we examined global no-flow I/R using a Langendorff model. No significant differences in coronary flow were observed between mPHGPx transgenic 
hearts and littermate control hearts at basal (in $\mathrm{ml} / \mathrm{min}$ : Control, 4.97 \pm 0.22 ; $\mathrm{mPHGPx}$, 4.58 \pm 0.36 ) and 30 minutes following $\mathrm{I} / \mathrm{R}$ (in $\mathrm{ml} / \mathrm{min}$ : Control, $3.15 \pm 0.22 ; \mathrm{mPHGPx}$, $3.84 \pm 0.50)$. MPHGPx transgenic hearts tended $(P<0.075)$ to have higher coronary flow rates 60 minutes following I/R (in ml/min: Control, 2.80 $\pm 0.20 ; \mathrm{mPHGPx}, 3.79 \pm 0.53$ ), and showed significantly $(P<0.05)$ higher flow rates 90 minutes following $\mathrm{l} / \mathrm{R}$ (in $\mathrm{ml} / \mathrm{min}$ : Control, 2.38 $\pm 0.15 ; \mathrm{mPHGPx}, 3.78 \pm 0.46)$. Following $\mathrm{I} / \mathrm{R}$, rates of contraction $(+\mathrm{dP} / \mathrm{dt})$ were significantly better in $\mathrm{mPHGPx}$ transgenic mice as compared to control mice, at 30 minutes into reperfusion (Figure 5.4A; $P<0.01$ ), 60 minutes into perfusion (Figure 5.4A; $P<0.05$ ), and 90 minutes into reperfusion (Figure 5.4A; $P<0.05$ ). In contrast, no significant differences were observed in rates of relaxation (-dP/dt; Figure 5.4B). Developed pressures (DP) were significantly better in mPHGPx transgenic mice as compared to controls, 30 and 60 minutes into reperfusion (Figure $5.4 \mathrm{C} ; P<0.05$ for both), and tended to be better 90 minutes into reperfusion (Figure 5.4C; $P<0.075$ ). Peak-systolic pressures (PSP) were significantly greater in mPHGPx transgenic mice as compared to control mice at all measured time points into reperfusion (Figure 5.4D; $P<0.05$ ), but no significant differences were observed in end-diastolic pressure (EDP; data not shown), indicating that the enhanced DP observed with mPHGPx overexpression was due solely to an increase in PSP. Examination of the rise in EDP resulting from ischemia (ischemic contracture) revealed a great deal of variance, but ultimately, no significant differences between control and mPHGPx transgenic (in $\mathrm{mm}$ $\mathrm{Hg}$ : Control, 12.04 \pm 4.17 ; mPHGPx, 15.99 \pm 3.36 ). These data indicate that $\mathrm{mPHGPx}$ transgenic overexpression enhances cardiac contractile function relative to control following global no-flow I/R insult, and that this preservation of function occurs primarily in the reperfusion period.

\section{Cardiac Necrosis Following Global Myocardial I/R}

Cellular damage was examined at various time points into the reperfusion period, via assessment of CK release. Preischemic (Pre) basal CK release did not differ 
between the two groups (Figure 5.5). CK release was significantly great in littermate control hearts after ischemia at 2 minutes into the reperfusion period (Figure 5.5; $P<0.01$ ), with no other time point showing significant differences. However, overall there was a trend for increased CK in the effluents of littermate control hearts at 1 and 3 minutes into the reperfusion period, as compared to mPHGPx transgenic hearts (Figure 5.5). These results indicate that mPHGPx overexpression was capable of protecting against I/R-initiated cellular disruption and damage in the myocardium.

\section{Oxidative Damage to Mitochondrial Lipids Following Global Myocardial I/R}

Maintenance of the mitochondrial phospholipid bilayer is critical for preservation of mitochondrial protein components, and appropriate membrane fluidity during I/R. Because it is involved in lipid peroxide scavenging, we examined whether mPHGPx overexpression could lessen I/R-associated lipid peroxidation in the mitochondrion, thus preserving membrane integrity. To index mitochondria-specific lipid peroxidation, we determined the content of two lipid peroxidation breakdown products, malondialdehyde (MDA) and 4-hydroxyalkenals (4-HNE), in total mitochondrial fractions following global $\mathrm{I} / \mathrm{R}$ insult. Figure 5.6 indicates that following global I/R, mPHGPx transgenic mice possessed approximately $40 \%$ less MDA and 4-HNE in the mitochondrial fraction as compared to littermate controls $(P<0.05)$. These data are in partial agreement with our previous studies examining the impact of adenoviral vector-mediated transgene delivery to neonatal cardiac myocytes subjected to simulated I/R (hypoxia/reoxygenation) [31] and indicate that mPHGPx transgenic overexpression provides protection to lipids in the mitochondrion. 


\section{Total Mitochondria ETC Complex Function Following Global Myocardial I/R}

Mitochondrial ETC complex function is altered with $\mathrm{l} / \mathrm{R}$ insult, which may occur as a result of increased ROS generation leading to ETC complex dysfunction. We used polarographic assessment to determine changes in total mitochondrial function following global myocardial I/R insult. Complex I, III, and IV activities were all significantly greater in total mitochondria from mPHGPx transgenic hearts following global myocardial I/R insult (Figure $5.7 ; \quad P<0.05$ for all three). These results indicate that $\mathrm{mPHGPx}$ overexpression protects mitochondrial function following global myocardial I/R insult, and that this protection is associated with preservation of ETC complexes I, III, and IV. We have previously reported that overexpression of mPHGPx via adenoviral vectors, preserves complex IV activity in neonatal cardiac myocytes following simulated I/R [31]. The results from our current study are in partial agreement with our previous studies and collectively indicate that mPHGPx transgenic overexpression provides protection to mitochondria function in a number of different models of cardiac I/R insult.

\section{Mitochondrial Subpopulation ETC Complex Function Following Global Myocardial I/R}

MPHGPx transgenic overexpression provides protection to ETC complex function in total mitochondria fractions following global myocardial $l / R$, but it is unclear whether this protection is uniform between spatially distinct mitochondrial subpopulations. Individual mitochondrial subpopulations were isolated, and flow cytometric analyses performed to verify the effectiveness of the isolation procedure. Assessment of forward-scattered light (FSC) was used to estimate size, while assessment of side-scattered light (SSC), was used to estimate mitochondrial complexity, both of which were based on a logarithmic scale. Individual mitochondria subpopulations were stained with MitoTracker Deep Red 633, which incorporates into intact mitochondria. In Figure A.8A, a typical dot plot, showing MitoTracker Deep Red 633 stained mitochondria is shown, with intact mitochondria indicated in blue and 
unstained debris indicated in green. Using this information, we gated the mitochondria (R1) to exclude unstained debris, and applied the R1 gate to analyses on the individual subpopulations. These analyses lend insight into relative morphological differences between the two subpopulations, and help to confirm success of the isolation procedure. To confirm differences in absolute mitochondria size, we included size calibration beads composed of microsphere suspensions ranging in size from $0.5 \mu \mathrm{M}$ to $6 \mu \mathrm{M}$ to serve as reliable size references. Using this approach, SSM were larger in size (FSC) and possessed greater internal complexity (SSC) as compared to IFM, which were smaller and more compact (Figures 5.8B and 5.8C). These results are consistent with previously published reports $[42,49,50]$.

As above, we used polarography to assess changes in ETC complex function following myocardial I/R insult, within spatially distinct mitochondrial subpopulations. MPHGPx overexpression enhanced ETC complexes I, III, and IV activities in SSM, following myocardial I/R insult, as compared to controls (Figure $5.8 \mathrm{D} ; P<0.05$, for all three). In contrast, mPHGPx overexpression enhanced only ETC complexes I and III activities in IFM, following myocardial I/R insult, as compared to controls (Figure 5.8E; $P<0.05$ for both). These results indicate that the protection provided by mPHGPx transgenic overexpression may be subject to spatial influence within the mitochondrion. Further, mPHGPx overexpression may provide SSM with greater protection than IFM, during myocardial $I / R$ insult. 


\section{DISCUSSION}

Myocardial I/R injury disrupts biochemical and physiological processes in the heart, and central to this disruption are ROS [10]. The main source of ROS during I/R is the ETC, which is highly susceptible to oxidative damage because many of its components possess iron-sulphur centers which act as targets for ROS [12]. As ETC components become damaged they promote injury to other mitochondrial components enhancing cellular dysfunction. Studies have indicated that antioxidant defense is crucial in protecting mitochondrial constituents from I/R-induced damage [16-18]. Because mitochondrial damage is a hallmark of $I / R$ injury, an emphasis has been placed on generating therapeutics designed to maintain/enhance key antioxidants specific to the mitochondrion. As a result, the current study was designed to test the hypothesis that mitochondria-specific overexpression of GPx4 could lessen oxidative damage resulting from myocardial $I / R$ insult, by preserving ETC complex function translating into enhanced cardiac contractile function. In an effort to understand the prophylactic contribution of mitochondria-specific GPx4 overexpression, we developed a novel transgenic mouse line that expresses GPx4 at a high level, solely in the mitochondrion (mPHGPx). Analysis of GPx4 expression in the mitochondria revealed dramatic increases in functionally active protein expression (Figure 5.2A). Further, utilization of an RT-PCR approach for transgene screening, indicated that the increase in GPx4 mRNA was similar in amount to other transgenic animals (hsp27) generated using a similar strategy [37] (Table 5.1). To confirm whether GPx4 overexpression was confined to the mitochondria, we isolated extracts from various cellular compartments and determined GPx4 protein expression. GPx4 protein analysis of mitochondria, cytosol, nuclear, and total homogenate from cardiac tissue confirmed a large increase in GPx4 protein specifically in the mitochondria (Figure 5.2B). A small signal was detected in the cytosolic fraction of mPHGPx transgenics, which was similar to our previous findings using adenoviral vector-mediated gene delivery of mPHGPx to rat neonatal cardiac myocytes [31]. As we reported previously, this small signal may be the result of mPHGPx proteins destined for the mitochondria that have not been fully processed and as a result are awaiting entry into the mitochondria. We verified our isolation 
procedures by examining the expression of compartment-specific proteins. Our results indicate that for the most part, the isolated fractions were pure, though slight contamination could be observed (Figure 5.2C). Thus one cannot rule out the potential for cross contamination during isolation. It is important to point out that $\mathrm{mPHGPx}$ expression exists primarily at contact sites between the inner and outer mitochondrial membranes, though it is possible in our transgenic mouse model, other submitochondrial locations may possess enhanced mPHGPx protein content. The $\mathrm{N}$ terminal presequence of the mPHGPx protein contains the features of a mitochondrial bipartite targeting signal which directs the protein to the intermembrane space [39]. Nevertheless, in certain cell types during development, mPHGPx expression patterns have been shown to be dynamic, moving from one submitochondrial site to another (Haraguchi). Future studies designed to determine the specific location(s) within the mitochondria, would be of interest. Because mitochondria exist as spatially distinct subpopulations, we examined whether mPHGPx overexpression occurred in a subpopulation-specific manner. SSM and IFM, possessed similar MPHGPx protein levels (Figure 5.2D), indicating that expression patterns did not favor either subpopulation.

It is well established that myocardial I/R promotes damage to cardiac proteins which results in contractile dysfunction [6]. In the current study, mPHGPx overexpression provided protection to cardiac contractile function during $\mathrm{I} / \mathrm{R}$, and this protection was subject to temporal influence (Figure 5.4). Specifically, we observed enhanced developed pressures (DP) in mPHGPx hearts at 30 and 60 minutes into reperfusion, with a trend of increase 90 minutes into reperfusion (Figure 5.4C). Rates of contraction $(+d P / d t)$ were significantly greater in $\mathrm{mPHGPx}$ transgenic hearts at all time points, but the degree of significance was greater at 30 minutes into reperfusion as compared to 60 and 90 minutes into reperfusion (Figure 5.4A). Taken together, these results indicate that $\mathrm{mPHGPx}$ presence enhanced cardiac contractile function proximally into the reperfusion period, to a greater extent than at distal time points. In contrast, no differences in rates of relaxation $(-\mathrm{dP} / \mathrm{dt})$ were observed in $\mathrm{mPHGPx}$ transgenic hearts at any time points (Figure 5.4B). Interestingly, peak systolic 
pressures (PSP) were greater in $\mathrm{mPHGPx}$ transgenic hearts at all time points into reperfusion as compared to controls (Figure 5.4D). It is not entirely clear why mPHGPx provided these particular patterns of cardiac protection, but overall these patterns seemed to influence protection in the contraction phase, rather than the relaxation phase, with a greater impact earlier in the reperfusion period. These results may be due to an overall decrease in oxidative stress during $I / R$, leading to preservation of myocyte viability and/or number. Because our examination of cardiac contractile performance included analyses solely at 30,60 , and 90 minutes, it is unclear whether mPHGPx overexpression provided contractile protection earlier in the reperfusion period. We previously observed decreased necrosis and apoptosis in neonatal cardiac myocytes infected with an adenoviral vector expressing mPHGPx that had been submitted to simulated I/R (hypoxia/reoxygenation) [31]. Examination of CK release following $I / R$ indicated a cardioprotective profile in $\mathrm{MPHGPx}$ hearts (Figure 5.5), confirming our previous findings. It is important to point out that the mechanistic function of $\mathrm{mPHGPx}$ is dependent on the use of the reducing equivalent glutathione (GSH). Myocardial I/R is associated with a decrease in the ratio of reduced:oxidized glutathione (GSH:GSSG) [2], thus it is possible that loss of GSH reducing capacity as opposed to enzymatic peroxide scavenging may be the limiting factor in the sequestering of injurious phospholipid hydroperoxides. Examination of combinatorial therapeutic approaches that couple mPHGPx overexpression with enhancement or preservation of GSH would be of interest for future studies.

MPHGPx is associated with subcellular membranes and is the primary enzymatic defense against oxidation of phospholipids in biomembranes [26]. Because reactive products derived from the oxidation of lipids promote deleterious effects on membrane constituents, we examined MDA and 4-HNE content in isolated mitochondria following myocardial I/R. Our results indicate that MDA and 4-HNE were collectively lower in mPHGPx mitochondria following myocardial I/R, as compared to controls (Figure 5.6). These results are in agreement with our previous findings, and confirm that, as expected, one locus of protection provided by mPHGPx is mitochondrial membranes [31]. Our results are in partial agreement with those of Ran et al. who observed 
decreases in liver total isoprostane levels in GPx4 transgenic mice that express the protein in numerous cellular compartments [33]. Enhanced scavenging of lipid hydroperoxides by mPHGPx may allow for the preservation of phospholipids within the mitochondrial bilayer which ultimately protects mitochondrial function by preserving ETC components and influencing mitochondria permeability transition pore (mPTP) dynamics. Cardiolipin, a phospholipid specific to the mitochondria, is easily oxidized and plays a role in maintaining cytochrome $C$ within the mitochondria [21]. Following cardiolipin oxidation, cytochrome $C$ can be released and facilitate apoptosome formation prior to cellular apoptosis [51]. It has been suggested that cardiolipin hydroperoxide is a specific target of mPHGPx action and the ability to preserve cardiolipin in the mitochondria may play a role in its mechanism of protection [21].

Due to the role played by mPHGPx in scavenging phospholipid hydroperoxides and preserving inner mitochondrial membrane integrity, we explored whether mitochondria specific GPx4 overexpression would be protective to ETC complexes. ETC complexes are surrounded by phospholipids in the inner mitochondrial membrane that contribute to their stability, integrity, and function. Many of these phospholipids are more easily prone to oxidation which in turn can damage the ETC. Overexpression of mPHGPx increased all ETC complex (complex I, III, IV) activities following myocardial $\mathrm{I} / \mathrm{R}$ as compared to control (Figure 5.7). These results are in partial agreement with our previous findings indicating that $\mathrm{mPHGPx}$ overexpression preserves complex IV activity following simulated I/R [31]. The disparity between our previous results and those from the current study may be due to several differences in the study designs. First, our previous study utilized rat neonatal cardiac myocytes, a relatively common model used in simulated I/R studies. Though the neonatal cardiac myocyte responds to the deleterious effects of simulated I/R, it is more resistant to hypoxic conditions, based in part on its greater use of glycolytic pathways for metabolism. As a result, the oxidative stress imposed may have been better tolerated in the neonatal cardiac myocyte, as compared to the adult cardiac myocyte, which is more reliant upon oxidative processes for metabolism. Further, one has to consider the difference in $\mathrm{I} / \mathrm{R}$ model systems employed, simulated I/R vs. whole heart I/R, which may provide different stresses and 
damage profiles. Our studies are somewhat in disagreement with those of Liang et al., who observed no differences with diquat exposure in transgenic mice that overexpress GPx4 in multiple cellular compartments [52]. A number of differences in study design may have contributed to the discrepant results. First, the GPx4 transgenic mouse utilized by Liang et al. displays different GPx4 expression patterns (lower) than the animals utilized in the current study, and they express the protein in multiple cellular compartments. It is possible that the GPx4 protein levels were not large enough to elicit a robust protective effect to the ETC, or that the enhanced GPx4 expression in other cellular compartments, such as the cytosol, may have lessened the oxidative stress presented to the mitochondria. It is has been previously reported that transgenic overexpression of GPx1 in multiple cellular compartments, such as the cytosol, may decrease cytosolic oxidant tone, which may impact oxidant levels within the cell [35]. Second, these authors utilized intraperitoneal injection of diquat to elicit an oxidative challenge to the liver, which is somewhat different than the current study, in which a global I/R model was used to elicit an oxidative challenge to the heart. Finally, these authors made their mitochondrial measurements following isolation of individual ETC complexes via blue native gel electrophoresis. This is in contrast to the current study, in which classic polarography using a Clark type electrode was employed to make ETC complex activity measurements in intact mitochondria. Nevertheless, the differences in results are interesting, and warrant further investigation.

Two mitochondrial subpopulations are present in the cardiac myocyte, interfibrillar mitochondria (IFM), which situate between the contractile apparatus, and subsarcolemmal mitochondria (SSM), which exist beneath the plasma membrane [53]. These two spatially distinct mitochondrial subpopulations are distinguished by specific cellular arrangements, separate functional properties, and differential responses to pathological conditions [42, 49, 53-57]. It has been suggested that the differential response observed in the two mitochondrial subpopulations during I/R may be due to variation in local oxygen tension within spatially distinct parts of the cell [58]. Because I/R impacts mitochondria subpopulations differently, we wanted to determine whether subpopulation-specific patterns of protection were provided by mPHGPx 
overexpression. Flow cytometric analyses revealed that in general, SSM appear to be larger and more complex than IFM, ranging in size from approximately $0.5 \mu \mathrm{m}$ to $3.0 \mu \mathrm{m}$ (Figure 5.8B). Overall, the IFM were smaller, less complex and less variable in size, ranging from $<0.5$ to $2.0 \mu \mathrm{m}$ (Figure $5.8 \mathrm{C}$ ). These findings were comparable to what others have found regarding morphological differences between the two populations $[42,49,50]$. Examination of ETC complex activities indicated that all components of the ETC (complex I, III, IV) were protected with mPHGPx overexpression in the SSM (Figure 5.8D). In contrast, only proximal ETC complexes (complex I, complex III) were protected with mPHGPx overexpression in the IFM (Figure 5.8E). Our results suggest that the protective profiles provided by mPHGPx overexpression are different in spatially distinct mitochondria subpopulations. This may be due in part to a difference in the susceptibility of spatially distinct mitochondria subpopulations to $I / R$, and in particular ETC complex IV, as reported previously [59].

In conclusion, we report for the first time, that mitochondria-specific transgenic overexpression of GPx4 in a mouse model provides protection to cardiac contractile function following myocardial $\mathrm{I} / \mathrm{R}$ insult. Further, the cardiac contractile protection provided by $\mathrm{mPHGPx}$ overexpression is associated with enhanced mitochondrial ETC complex activities. These findings provide further rationale for the use of mPHGPx as a therapeutic protectant against I/R-induced cardiac injury. 


\section{ACKNOWLEDGEMENTS}

This work was supported by American Heart Association Beginning Grant-In-Aid \#0665237B (J. M. H.). We would like to thank Dr. Christopher Cuff and the contributions from the West Virginia University Flow Cytometry Core Facility. Flow cytometry studies were supported in part by grants \#RR020866 and \#RR16440. We would also like to thank Dr. Peter Mathers and the contributions from the West Virginia University Transgenic Animal Core Facility. The West Virginia University Transgenic Animal Core Facility was supported in part by grants \#RR015574 and \#RR016440. 


\section{REFERENCES}

[1] Bluhm, W. F.; Martin, J. L.; Mestril, R.; Dillmann, W. H. Specific heat shock proteins protect microtubules during simulated ischemia in cardiac myocytes. Am. J. Physiol. 275:H2243-H2249; 1998.

[2] Ji, L. L.; Fu, R. G.; Waldrop, T. G.; Liu, K. J.; Swartz, H. M. Myocardial response to regional ischemia and reperfusion in vivo in rat heart. Can. J. Physiol. Pharmacol. 71:811-817; 1993.

[3] Park, Y.; Kanekal, S.; Kehrer, J. P. Oxidative changes in hypoxic rat heart tissue. Am. J. Physiol. 260:H1395-H1405; 1992.

[4] Sevanian, A.; McLeod, L. L. Lipid peroxidation and modification of lipid composition in an endothelial cell model of ischemia and reperfusion. Free Radic. Biol. Med. 23:680-694; 1997.

[5] Simpson, P. J.; Lucchesi, B. R. Free radicals and myocardial ischemia and reperfusion injury. J. Lab. Clin. Med. 110:95-100; 1987.

[6] Suzuki, S.; Kaneko, M.; Chapman, D. C.; Dhalla, N. S. Alterations in cardiac contractile proteins due to oxygen free radicals. Biochim. Biophys. Acta 1074:95-100; 1991.

[7] Ferrari, R.; Ceconi, C.; Curello, S.; Cargnoni, A.; De Giuli, F.; Visioli, O. Occurrence of oxidative stress during myocardial reperfusion. Mol. Cell. Biochem. 111:61-69; 1992.

[8] Guarnieri, C.; Ceconi, C.; Muscari, C.; Flamigni, F. Influence of oxygen radicals on heart metabolism. In: Caldarera, C. M.; Harris, P., eds. Advances in studies on heart metabolism. Bologna: Cooperative Libraria Universitaria Editrice; 1982: 423-431.

[9] Nohl, $\mathrm{H}$. The biochemical mechanism of the formation of reactive oxygen species in heart mitochondria. In: Caldarera, C. M.; Harris, P., eds. Advances in Studies on Heart Metabolism. Bologna: Cooperative Libraria Universitaria Editrice; 1982: 413-421.

[10] Downey, J. M. Free radicals and their involvement during long-term myocardial ischemia and reperfusion. Annual review of physiology 52:487-504; 1990.

[11] Zweier, J. L.; Flaherty, J. T.; Weisfeldt, M. L. Direct measurement of free radical generation following reperfusion of ischemic myocardium. Proceedings of the National Academy of Sciences of the United States of America 84:1404-1407; 1987. 
[12] Newsholme, E. A.; Leech, A. R. Biochemistry for the medical sciences. Chicester: John Wiley and Sons; 1983.

[13] Fridovich, I. Superoxide radical and superoxide dismutases. Ann. Rev. Biochem. 64:97-112; 1995.

[14] McCord, J. M. Free radicals and myocardial ischemia: overview and outlook. Free Rad. Biol. Med. 4:9-14; 1988.

[15] Zweier, J. L. Measurement of superoxide-derived free radicals in reperfused heart. J. Biol. Chem. 263:1353-1357; 1988.

[16] Haramaki, N.; Stewart, D. B.; Aggarwal, S.; Ikeda, H.; Reznick, A. Z.; Packer, L. Networking antioxidants in the isolated rat heart are selectively depleted by ischemiareperfusion. Free Rad. Biol. Med. 25:329-339; 1998.

[17] Dhaliwal, H.; Kirshenbaum, L. A.; Randhawa, A. K.; Singal, P. K. Correlation between antioxidant changes during hypoxia and recovery on reoxygenation. Am. J. Physiol. 261:H632-H638; 1991.

[18] Ferrari, R.; Ceconi, C.; Curello, S.; Guarnieri, G.; Caldarera, C. M.; Albertini, A.; Visioli, O. Oxygen-mediated myocardial damage during ischemia and reperfusion: role of the cellular defense against oxygen toxicity. J. Mol. Cell. Cardiol. 17:937-945; 1985.

[19] Arai, M.; Imai, H.; Koumura, T.; Yoshida, M.; Emoto, K.; Umeda, M.; Chiba, N.; Nakagawa, Y. Mitochondrial phospholipid hydroperoxide glutathione peroxidase plays a major role in preventing oxidative injury to cells. J. Biol. Chem. 274:4924-4933; 1999.

[20] Ji, L. L.; Leeuwenburgh, C.; Leichtweis, S.; Gore, M.; Fiebig, R.; Hollander, J.; Bejma, J. Oxidative stress and aging. Role of exercise and its influences on antioxidant systems. Annals of the New York Academy of Sciences 854:102-117; 1998.

[21] Imai, H.; Nakagawa, Y. Biological significance of phospholipid hydroperoxide glutathione peroxidase (PHGPx, GPx4) in mammalian cells. Free radical biology \& medicine 34:145-169; 2003.

[22] Godeas, C.; Sandri, G.; Panfili, E. Distribution of phospholipid hydroperoxide glutathione peroxidase (PHGPx) in rat testis mitochondria. Biochim. Biophys. Acta 1191:147-150; 1994.

[23] Ursini, F.; Maiorino, M.; Gregolin, C. The selenoenzyme phospholipid hydroperoxide glutathione peroxidase. Biochimica et Biophysica Acta 839:62-70; 1985.

[24] Schnurr, K.; Belkner, J.; Ursini, F.; Schewe, T.; Kuhn, H. The selenoenzyme phospholipid hydroperoxide glutathione peroxidase controls the activity of the 15- 
lipoxygenase with complex substrates and preserves the specificity of the oxygenation products. J. Biol. Chem. 271:4653-4658; 1996.

[25] Ursini, F.; Maiorino, M.; Brigelius-Flohe, R.; Aumann, K. D.; Rovery, A.; Schomburg, D.; Flohe, L. Diversity of glutathione peroxidases. Methods Enzymol. 252:38-83; 1995.

[26] Thomas, J. P.; Maiorino, M.; Ursini, F.; Girotti, A. W. Protective action of phospholipid hydroperoxide glutathione peroxidase against membrane-damaging lipid peroxidation. J. Biol. Chem. 265:454-461; 1990.

[27] Esterbauer, H.; Schaur, R. J.; Zollner, H. Chemistry and biochemistry of 4hydroxynonenal, malondialdehyde, and related aldehydes. Free Rad. Biol. Med. 11:81128; 1991.

[28] Poot, M.; Verkerk, A.; Koster, J. F.; Esterbauer, H.; Jongkind, J. F. Reversible inhibition of DNA and protein synthesis by cumene hydroperoxide and 4hydroxynonenal. Mech. Age. Dev. 43:1-9; 1988.

[29] Chen, J.; Henderson, G. I.; Freeman, G. L. Role of 4-hydroxynonenal in modification of cytochrome $\mathrm{c}$ oxidase in ischemia/reperfused rat heart. J. Moll. Cell. Cardiol. 33:1919-1927; 2001.

[30] Nomura, K.; Imai, H.; Koumura, T. A., M.; Nakagawa, Y. Mitochondrial phospholipid hydroperoxide glutathione peroxidase suppresses apoptosis mediated by a mitochondrial death pathway. J. Biol. Chem. 274:29294-29302; 1999.

[31] Hollander, J. M.; Lin, K. M.; Scott, B. S.; Dillmann, W. H. Overexpression of PHGPx and HSP60/10 protects against ischemia-reoxygenation injury. Free Rad. Biol. Med. 35:742-751; 2003.

[32] Yant, L. J.; Ran, Q.; Rao, L.; Van Remmen, H.; Shibatani, T.; Belter, J. G.; Motta, L.; Richardson, A.; Prolla, T. A. The selenoprotein GPX4 is essential for mouse development and protects from radiation and oxidative damage insults. Free radical biology \& medicine 34:496-502; 2003.

[33] Ran, Q.; Liang, H.; Gu, M.; Qi, W.; Walter, C. A.; Roberts, L. J., 2nd; Herman, B.; Richardson, A.; Van Remmen, $H$. Transgenic mice overexpressing glutathione peroxidase 4 are protected against oxidative stress-induced apoptosis. The Journal of biological chemistry 279:55137-55146; 2004.

[34] Ran, Q.; Liang, H.; Ikeno, Y.; Qi, W.; Prolla, T. A.; Roberts, L. J., 2nd; Wolf, N.; VanRemmen, $\mathrm{H}_{\text {.; }}$ Richardson, A. Reduction in glutathione peroxidase 4 increases life 
span through increased sensitivity to apoptosis. The journals of gerontology 62:932942; 2007.

[35] McClung, J. P.; Roneker, C. A.; Mu, W.; Lisk, D. J.; Langlais, P.; Liu, F.; Lei, X. G. Development of insulin resistance and obesity in mice overexpressing cellular glutathione peroxidase. Proceedings of the National Academy of Sciences of the United States of America 101:8852-8857; 2004.

[36] He, H.; Giordano, F. J.; Hilal-Dandan, R.; Choi, D.; Rockman, H. A.; McDonough, P. M.; Bluhm, W. F.; Meyer, M.; Sayen, M. R.; Swanson, E.; Dillmann, W. H. Overexpression of the rat sarcoplasmic reticulum $\mathrm{Ca}^{2+}$ ATPase gene in the heart of transgenic mice accelerates calcium transients and cardiac relaxation. J. Clin. Invest. 100:380-389; 1997.

[37] Hollander, J. M.; Martin, J. L.; Belke, D. D.; Scott, B. T.; Swanson, E.; Krishnamoorthy, V.; Dillmann, W. H. Overexpression of wild-type heat shock protein 27 and a nonphosphorylatable heat shock protein 27 mutant protects against ischemia/reperfusion injury in a transgenic mouse model. Circulation 110:3544-3552; 2004.

[38] Marber, M. S.; Mestrill, R.; Chi, S.; Sayen, R. S.; Yellon, D. M.; Dillmann, W. H. Overexpression of the rat inducible $70-\mathrm{kD}$ heat stress protein in a transgenic mouse increases the resistance of the heart to ischemic injury. J. Clin. Invest. 95:1446-1456; 1995.

[39] Pushpa-Rekha, T. R.; Burdsall, A. L.; Oleksa, L. M.; Chisolm, G. M.; Driscoll, D. M. Rat phospholipid-hydroperoxide glutathione peroxidase. CDNA cloning and identification of multiple transcription and translation start sites. The Journal of biological chemistry 270:26993-26999; 1995.

[40] Niwa, H.; Yamamura, K. I.; Miyazaki, J. I. Efficient selection for high-expression transfectants with a novel eukaryotic vector. Gene (Amst.) 108:193-200; 1991.

[41] Trost, S. U.; Omens, J. H.; Karlon, W. J.; Meyer, M.; Mestrill, R.; Covell, J. W.; Dillmann, W. H. Protection against myocardial dysfunction after a brief ischemic period in transgenic mice expressing inducible heat shock protein 70. J. Clin. Invest. 101:855862; 1998.

[42] Palmer, J. W.; Tandler, B.; Hoppel, C. L. Biochemical properties of subsarcolemmal and interfibrillar mitochondria isolated from rat cardiac muscle. The Journal of biological chemistry 252:8731-8739; 1977. 
[43] Moghaddas, S.; Stoll, M. S.; Minkler, P. E.; Salomon, R. G.; Hoppel, C. L.; Lesnefsky, E. J. Preservation of cardiolipin content during aging in rat heart interfibrillar mitochondria. The journals of gerontology 57:B22-28; 2002.

[44] Bradford, M. A rapid and sensitive method for quantitation of microgram quantities of protein utilizing the principle of protein dye binding. Anal. Biochem. 72:248256; 1976.

[45] Laemmli, U. K. Cleavage of structural proteins during the assembly of the head of bacteriophage T4. Nature 227:680-685; 1970.

[46] Maiorino, M.; Gregolin, C.; Ursini, F. Phospholipid hydroperoxide glutathione peroxidase. Methods Enzymol 186:448-457; 1990.

[47] Lei, X. G.; Evenson, J. K.; Thompson, K. M.; Sunde, R. A. Glutathione peroxidase and phospholipid hydroperoxide glutathione peroxidase are differentially regulated in rats by dietary selenium. J. Nutri. 125:1438-1446; 1995.

[48] Hofhaus, G.; Shakeley, R. M.; Attardi, G. Use of polarography to detect respiration defects in cell cultures. Methods Enzymol. 264:476-483; 1996.

[49] Koves, T. R.; Noland, R. C.; Bates, A. L.; Henes, S. T.; Muoio, D. M.; Cortright, R. N. Subsarcolemmal and intermyofibrillar mitochondria play distinct roles in regulating skeletal muscle fatty acid metabolism. Am J Physiol Cell Physiol 288:C1074-1082; 2005.

[50] Shimada, T.; Horita, K.; Murakami, M.; Ogura, R. Morphological studies of different mitochondrial populations in monkey myocardial cells. Cell and tissue research 238:577-582; 1984.

[51] Ott, M.; Robertson, J. D.; Gogvadze, V.; Zhivotovsky, B.; Orrenius, S. Cytochrome $\mathrm{c}$ release from mitochondria proceeds by a two-step process. Proceedings of the National Academy of Sciences of the United States of America 99:1259-1263; 2002.

[52] Liang, H.; Van Remmen, H.; Frohlich, V.; Lechleiter, J.; Richardson, A.; Ran, Q. Gpx4 protects mitochondrial ATP generation against oxidative damage. Biochemical and biophysical research communications 356:893-898; 2007.

[53] Riva, A.; Tandler, B.; Loffredo, F.; Vazquez, E.; Hoppel, C. Structural differences in two biochemically defined populations of cardiac mitochondria. American journal of physiology 289:H868-872; 2005. 
[54] Lesnefsky, E. J.; Slabe, T. J.; Stoll, M. S.; Minkler, P. E.; Hoppel, C. L. Myocardial ischemia selectively depletes cardiolipin in rabbit heart subsarcolemmal mitochondria. American journal of physiology 280:H2770-2778; 2001.

[55] Ritov, V. B.; Menshikova, E. V.; He, J.; Ferrell, R. E.; Goodpaster, B. H.; Kelley, D. E. Deficiency of subsarcolemmal mitochondria in obesity and type 2 diabetes. Diabetes 54:8-14; 2005.

[56] Suh, J. H.; Heath, S. H.; Hagen, T. M. Two subpopulations of mitochondria in the aging rat heart display heterogeneous levels of oxidative stress. Free radical biology \& medicine 35:1064-1072; 2003.

[57] Takahashi, M.; Hood, D. A. Protein import into subsarcolemmal and intermyofibrillar skeletal muscle mitochondria. Differential import regulation in distinct subcellular regions. The Journal of biological chemistry 271:27285-27291; 1996.

[58] Skulachev, V. P. Mitochondrial filaments and clusters as intracellular powertransmitting cables. Trends in biochemical sciences 26:23-29; 2001.

[59] Lesnefsky, E. J.; Chen, Q.; Slabe, T. J.; Stoll, M. S.; Minkler, P. E.; Hassan, M. O.; Tandler, B.; Hoppel, C. L. Ischemia, rather than reperfusion, inhibits respiration through cytochrome oxidase in the isolated, perfused rabbit heart: role of cardiolipin. American journal of physiology 287:H258-267; 2004. 


\section{Figure 5.1}

A
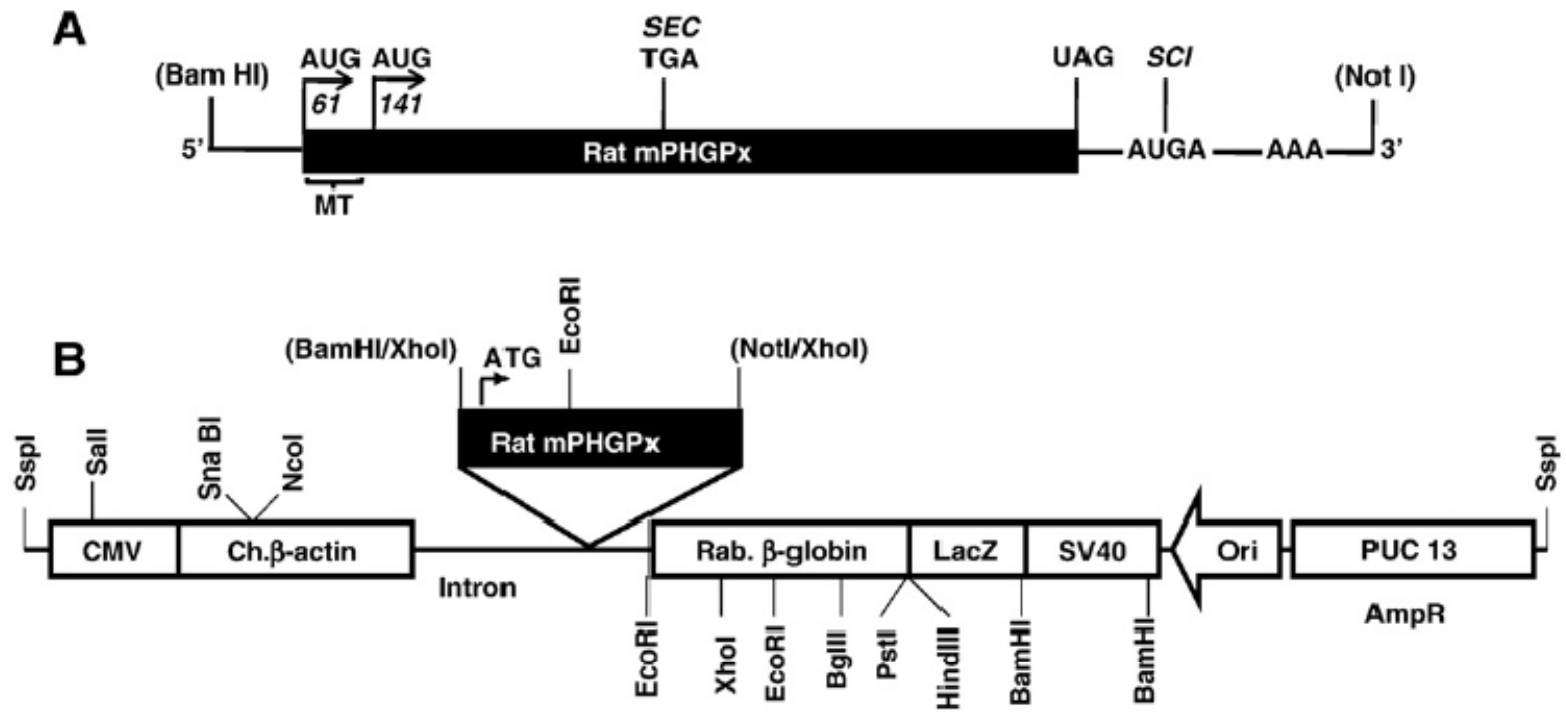
Figure 5.1. MPHGPX transgenic mouse construction. Generation of $\mathrm{mPHGPx}$ transgenic mice was accomplished by inserting the rat mPHGPx cDNA (approximately $870 \mathrm{bp}$ ) into the plasmid pCAGGS via blunt end ligation. (A) Schematic of the rat mPHGPx cDNA fragment that was inserted into the plasmid pCAGGS. An AUG start codon at $61 \mathrm{bp}$ precedes the mitochondria targeting sequence (MT). The AUG start codon at $141 \mathrm{bp}$ indicates the mPHGPx translational start. The selenocysteine (SEC) codon is indicated as a TGA at position (277-279 bp). The selenocysteine ( $S C l)$ insertion sequence which is required for SEC incorporation is indicated as AUGA and is located in the 3'-UTR. The polyadenylation signal in the 3'UTR is indicated as AAA. (B) The pCAGGS vector places the mPHGPX gene under the control of the human cytomegalovirus (CMV) immediate early enhancer and chicken $\beta$-actin (Ch. $\beta$-actin) promoter with first intron. The approximately 870-bp rat mPHGPx cDNA was inserted into the Xhol cloning site of $\mathrm{pCAGGS}$ as a BamHI/Notl fragment. The chimeric transgene was cut out of the plasmid by Sspl and BamHI digestion, purified, and used to generate transgenic mice. 
Figure 5.2

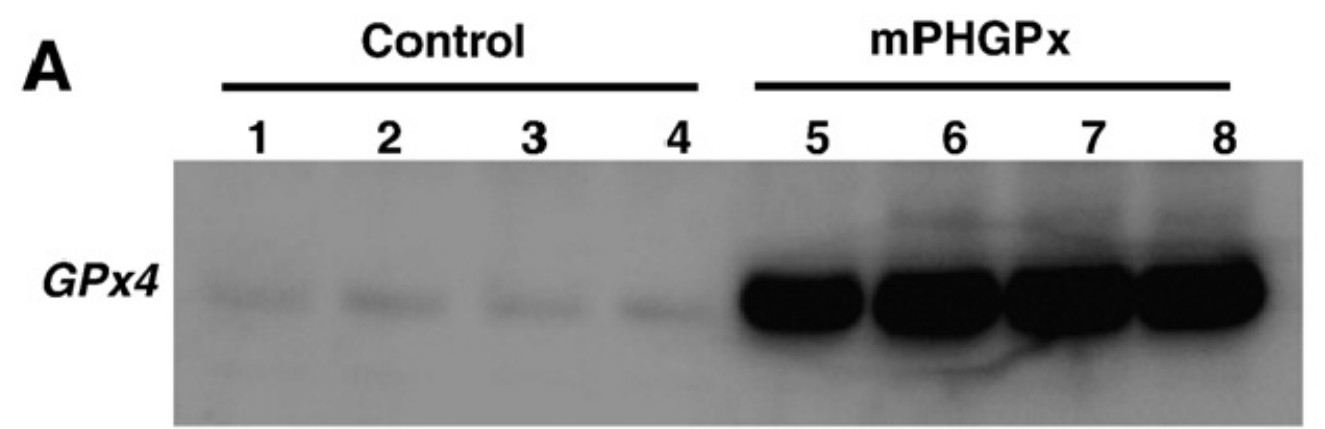

Grp75

B
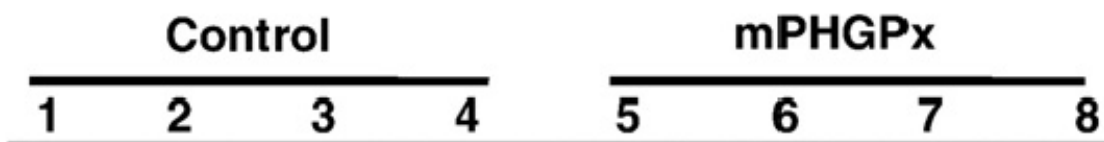

GPX4

Mi Cy $\mathrm{Nu}$ Hom Mi Cy $\mathrm{Nu}$ Hom

C

Oct-1

GAPDH

CycD

D

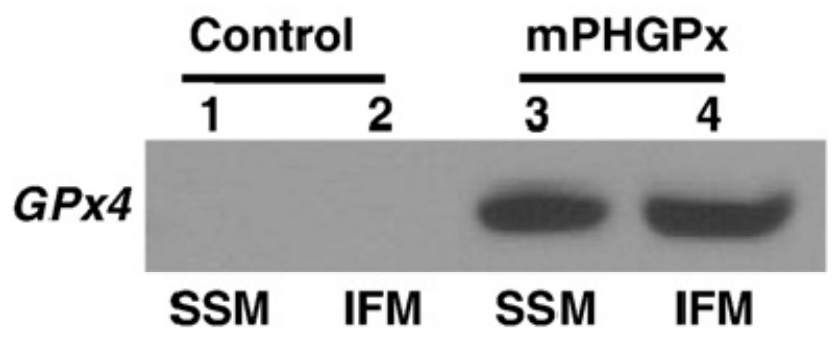


Figure 5.2. MPHGPX protein analyses. (A) Representative Western blot analysis of GPx4 (PHGPx) protein expression in isolated total heart mitochondria from mPHGPx Line 1 transgenic positive mice and negative littermate controls. Lanes 1-4 are negative littermate controls, and Lanes 5-8 are mPHGPx transgenic positives. A representative Western blot analysis of Grp75 (mthsp75) is included as a loading control. (B) Representative Western blot analysis of GPx4 (PHGPx) protein expression in various cellular compartments of mPHGPx transgenic mouse heart and littermate control mouse heart. $\mathrm{Mi}$, mitochondria; $\mathrm{Cy}$, cytosol; $\mathrm{Nu}$, nucleus; Hom, total homogenate. Lanes 1-4 are from a negative littermate control, and Lanes 5-8 are from a mPHGPx transgenic positive. (C) Representative Western blot analysis of compartment-specific markers for verification of subcellular fractionation. Mi, mitochondria (CycD); Cy, cytosol (GAPDH); $\mathrm{Nu}$, nucleus (Oct-1). (D) Representative Western blot analysis of GPx4 (PHGPx) protein expression in individual mitochondrial subpopulations of mPHGPx transgenic mouse heart and littermate control mouse heart. SSM, subsarcolemmal mitochondria; IFM, interfibrillar mitochondria. Lanes 1-2 are from a negative littermate control, and Lanes 3-4 are from a mPHGPx transgenic positive. 
Figure 5.3

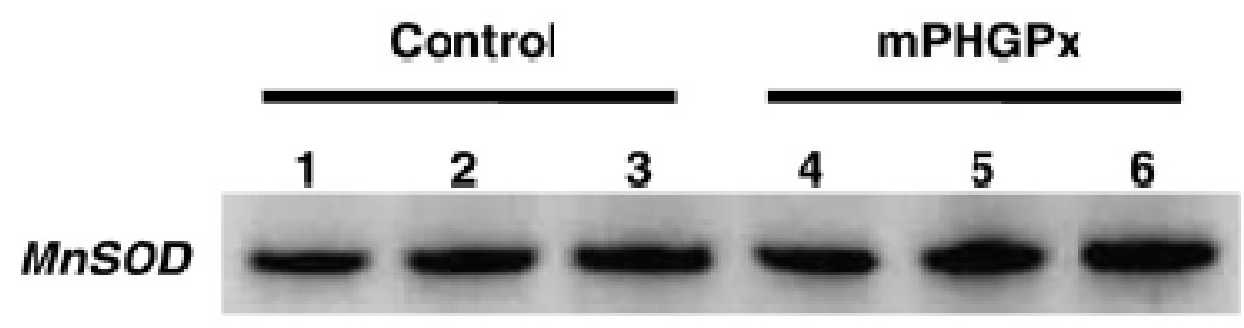

CUZnSOD

$$
\text { GPX1 }
$$

Catalase

GAPDH
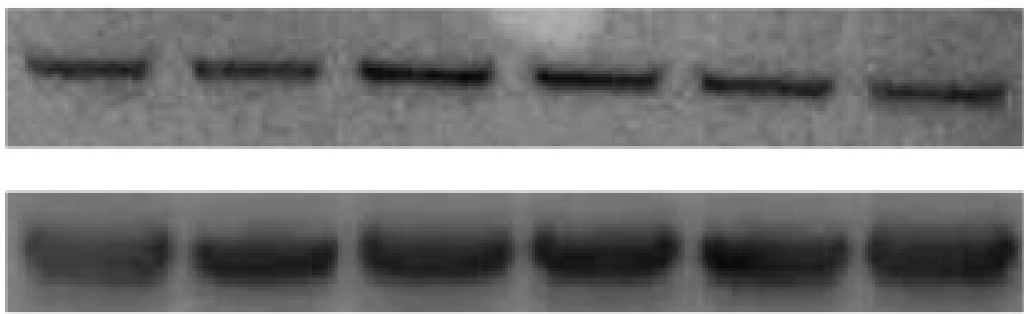
Figure 5.3. Antioxidant enzyme protein analyses. Representative Western blot analyses of mitochondrial superoxide dismutase (MnSOD), cytosolic SOD (CuZnSOD), glutathione peroxidase (GPx1), and catalase protein expression in total heart homogenate from mPHGPx transgenic positive mice and negative littermate controls. Lanes 1-3 are negative littermate controls, and Lanes 4-6 are mPHGPx transgenic positives. A representative Western blot analysis of GAPDH is included below as a loading control. 
Figure 5.4
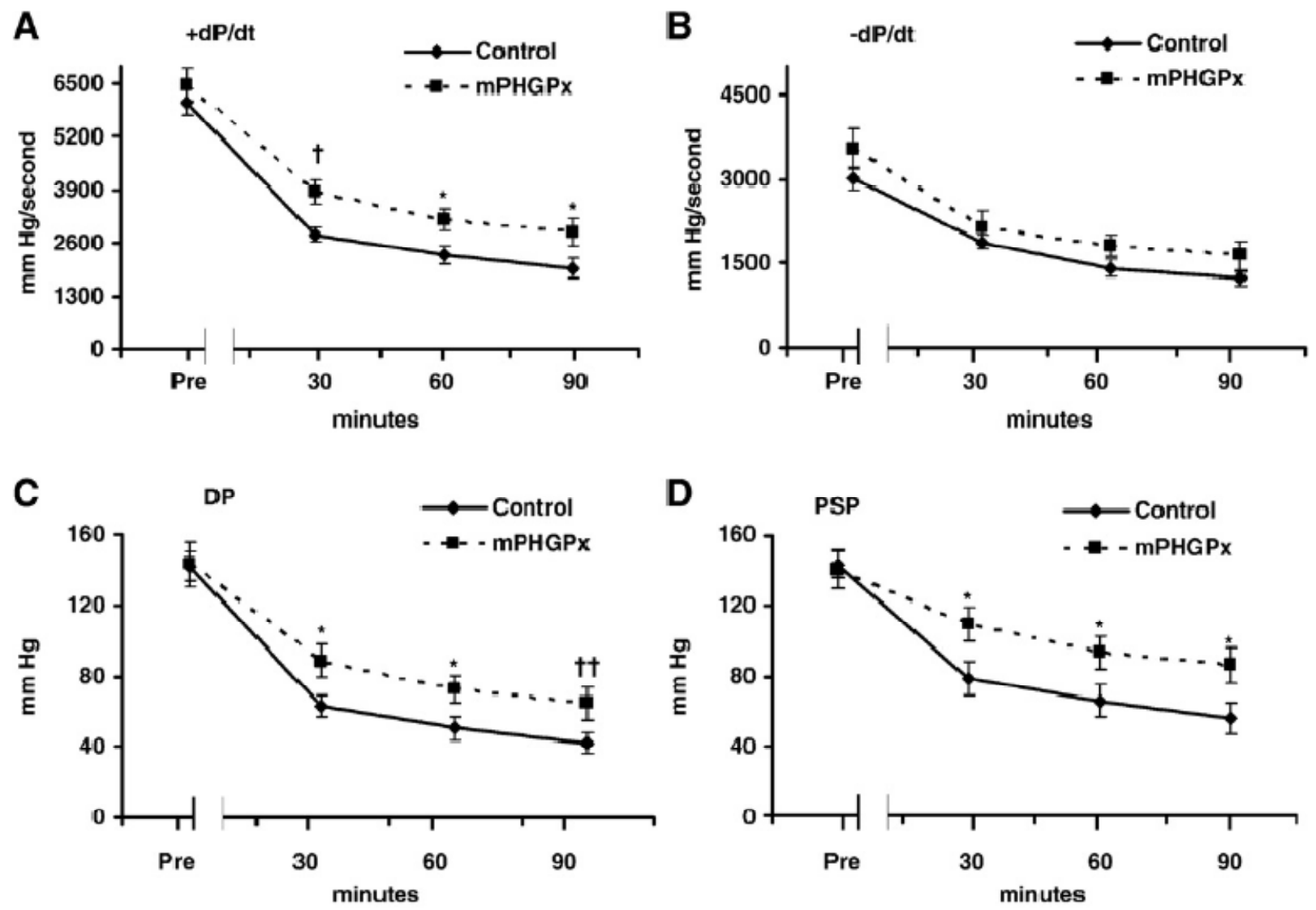
Figure 5.4. Cardiac contractile function following I/R. (A) Rate of contraction $(+d P / d t)$ in $\mathrm{mPHGPx}$ transgenic and littermate negative controls 30,60 , and 90 min following global I/R insult. (B) Rate of relaxation (-dP/dt) in mPHGPx transgenic and littermate negative controls 30, 60, and 90 min following global I/R insult. (C) Developed pressure (DP) in mPHGPx transgenic and littermate negative controls 30,60 , and 90 min following global I/R insult. (D) Peak systolic pressure (PSP) in mPHGPx transgenic and littermate negative controls 30,60 , and $90 \mathrm{~min}$ following global I/R insult. All functional measurements were performed in Langendorffperfused hearts. Rates of contraction $(+d P / d t)$ and rates of relaxation ($d P / d t)$ are expressed as $\mathrm{mm} \mathrm{Hg} /$ second. DP and PSP are expressed as $\mathrm{mm} \mathrm{Hg}$. Values are mean $\pm \mathrm{SE} ; N=8$ for each group. $* P<0.05$ for mPHGPx transgenic vs littermate control. $\dagger P<0.01$ for $\mathrm{mPHGPx}$ transgenic vs littermate control. $\dagger \dagger P<0.075$ for $\mathrm{mPHGPx}$ transgenic vs littermate control. 
Figure 5.5

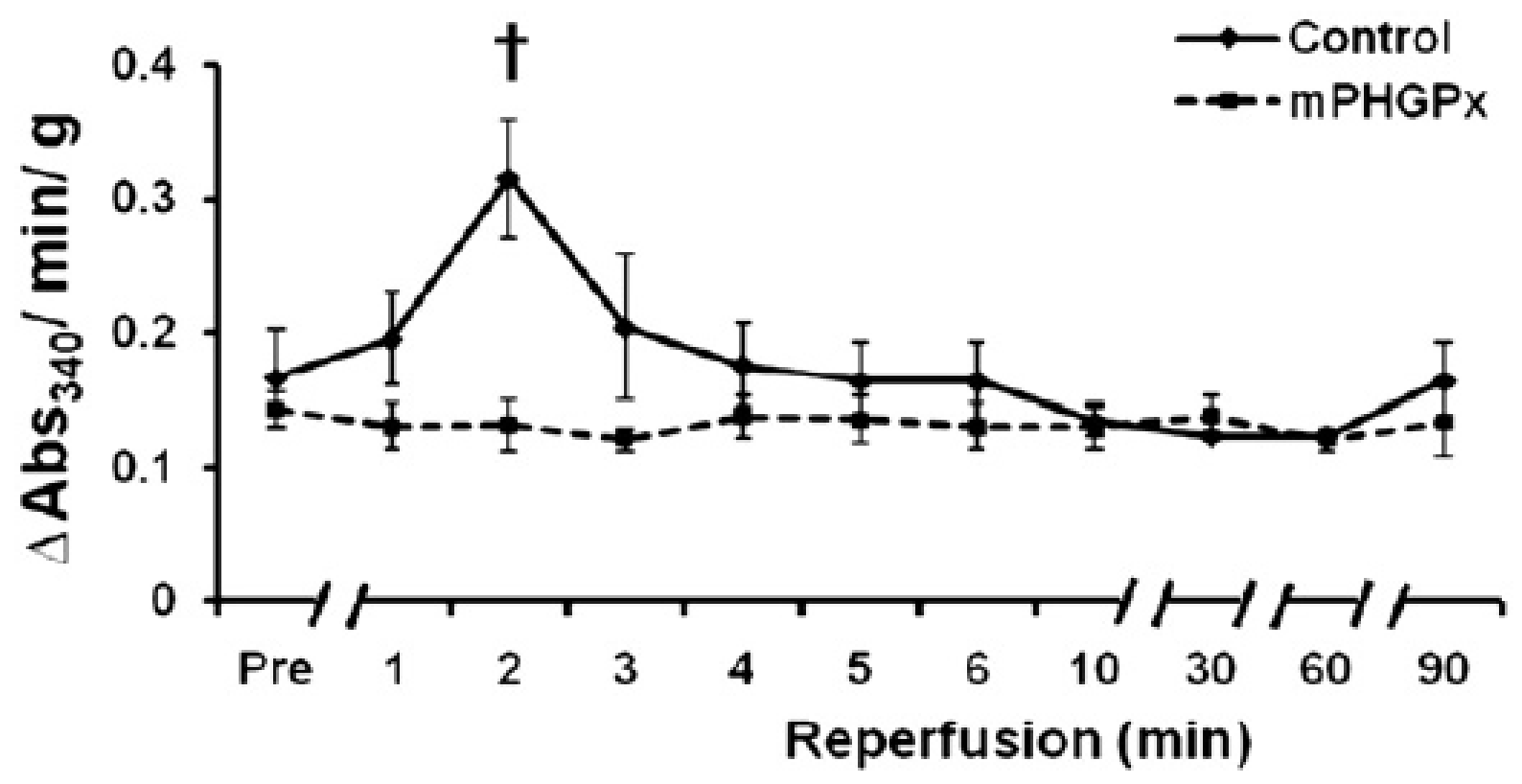


Figure 5.5. Creatine kinase release following I/R. Creatine kinase (CK) release was measured in effluents from reperfused hearts at various time points after ischemia. Measurements were taken prior to ischemia (Pre), 1, $2,3,4,5,6,10,30,60$, and 90 min into the reperfusion period. Values are mean $\pm \mathrm{SE} ; N=4$ for each group. $\dagger P<0.01$ for mPHGPx transgenic vs littermate control. 
Figure 5.6

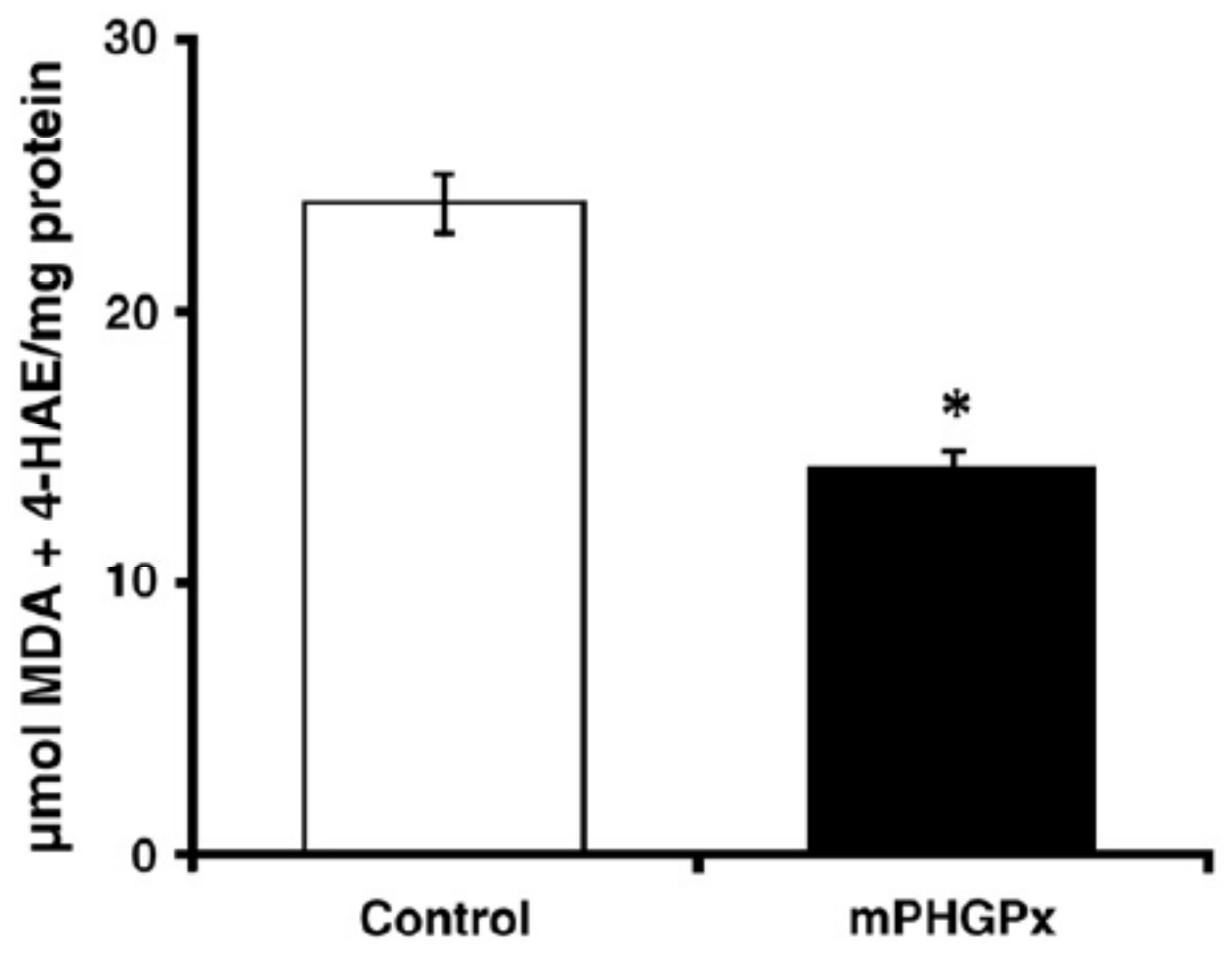


Figure 5.6. Total mitochondria lipid peroxidation content following I/R. Total mitochondria fractions were isolated from mPHGPX transgenic and littermate control hearts following global $\mathrm{I} / \mathrm{R}$ insult, and lipid peroxidation measurements performed. Assessment of the lipid peroxidation byproducts malondialdehyde (MDA) and 4-hydroxyalkenals (4-HAE) was performed using a colorimetric assay and spectrophotometric measurement at $586 \mathrm{~nm}$. Sample values were compared against a standard curve of known 4-HAE and MDA, and data are expressed as umole MDA + 4-HAE per $\mathrm{mg}$ of protein. Values are expressed as mean $\pm \mathrm{SE} ; N=5$ for each group. $* P<0.05$ for mPHGPx vs control. 
Figure 5.7

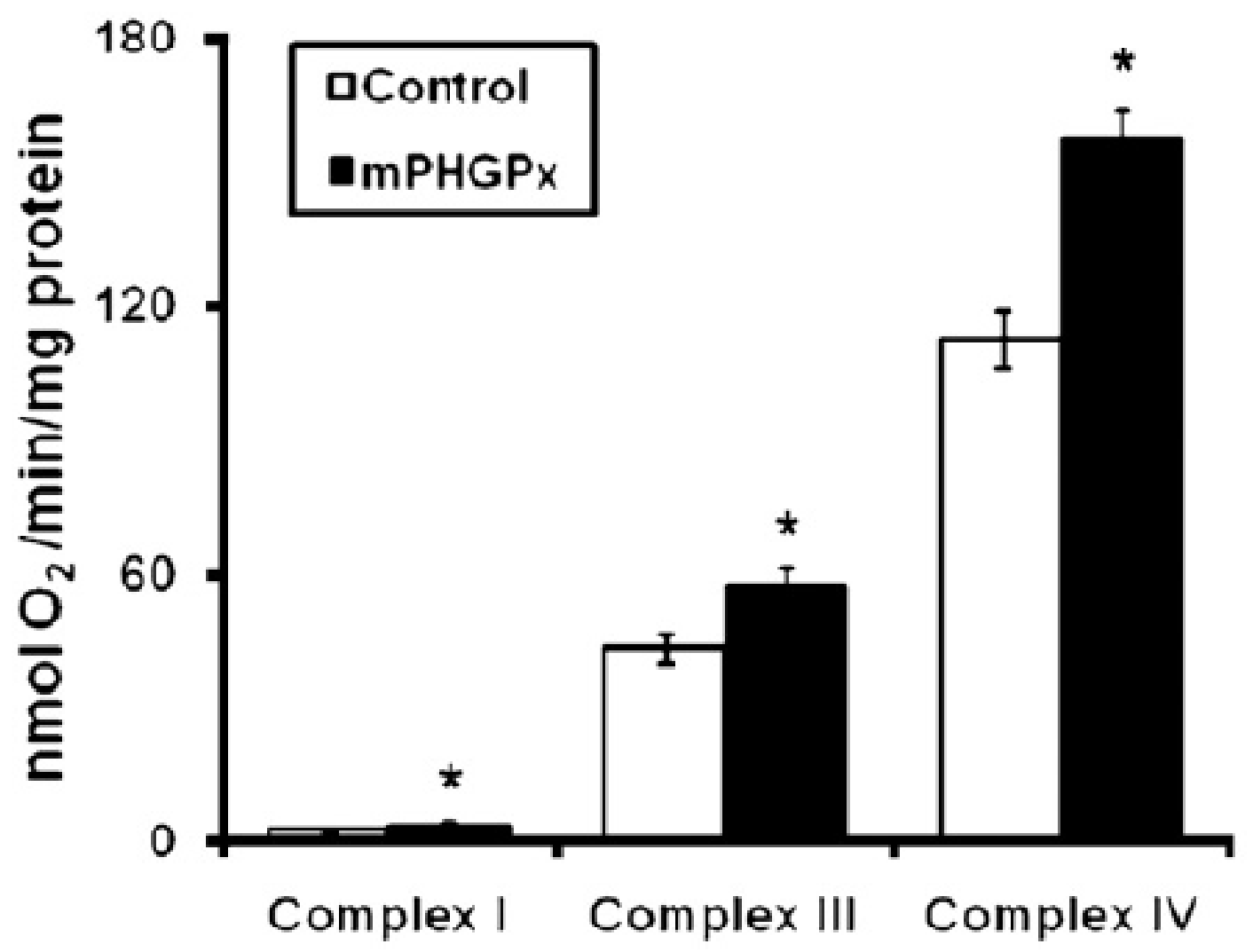


Figure. 5.7. Mitochondria electron transport chain (ETC) function following I/R. Total mitochondria fractions were isolated from $\mathrm{mPHGPx}$ transgenic and littermate control hearts following global $I / R$ insult, and polarographic measurements performed to index oxygen consumption. Activities of individual ETC complexes were defined as the rate of oxygen consumed in the presence of specific substrates, glutamate/malate (complex I), succinate (complex III), and ascorbate $/ N, N, N^{\prime}, N^{\prime}$-tetramethyl-pphenylenediamine (complex IV), and were calculated as the fraction that was sensitive to the specific inhibitors rotenone (complex I), antimycin (complex III), and sodium cyanide (complex IV). Values are mean $\pm \mathrm{SE}$; $N=8$ for each group. $* P<0.05$ for mPHGPx vs control. 
Figure 5.8

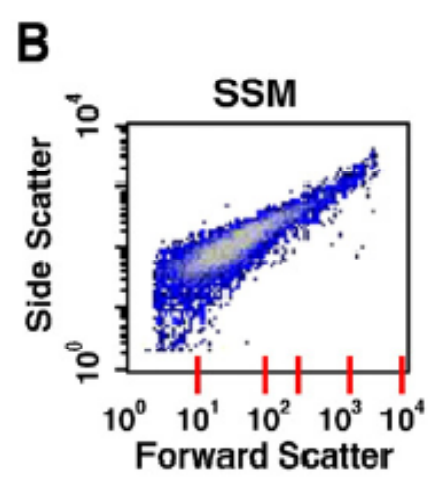

D

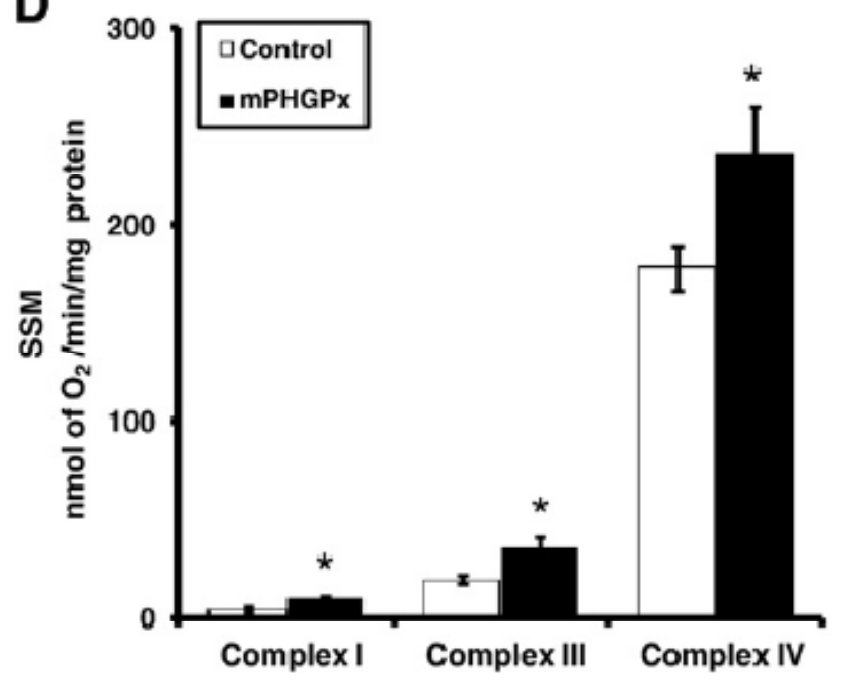

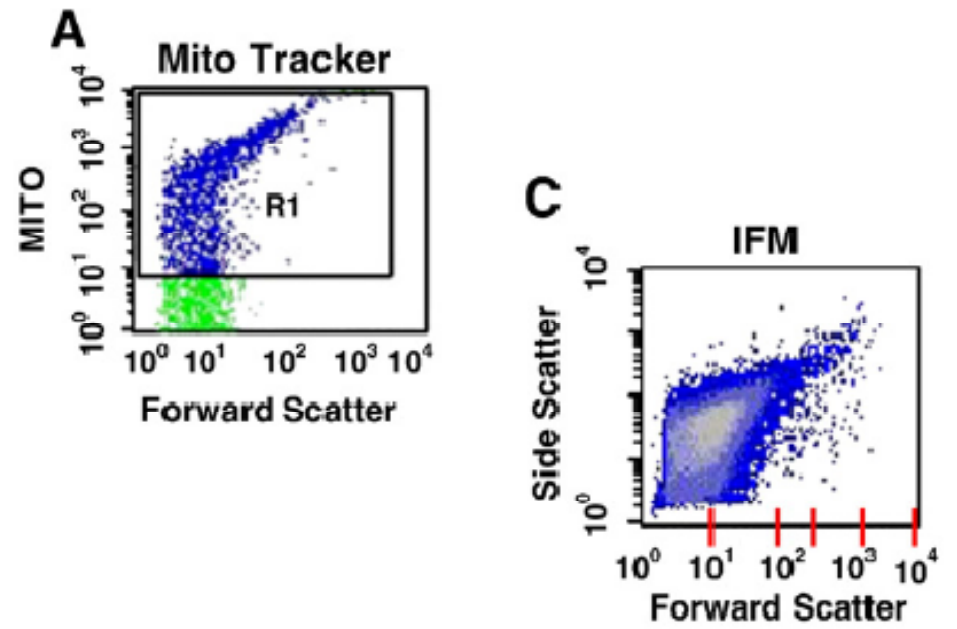

E

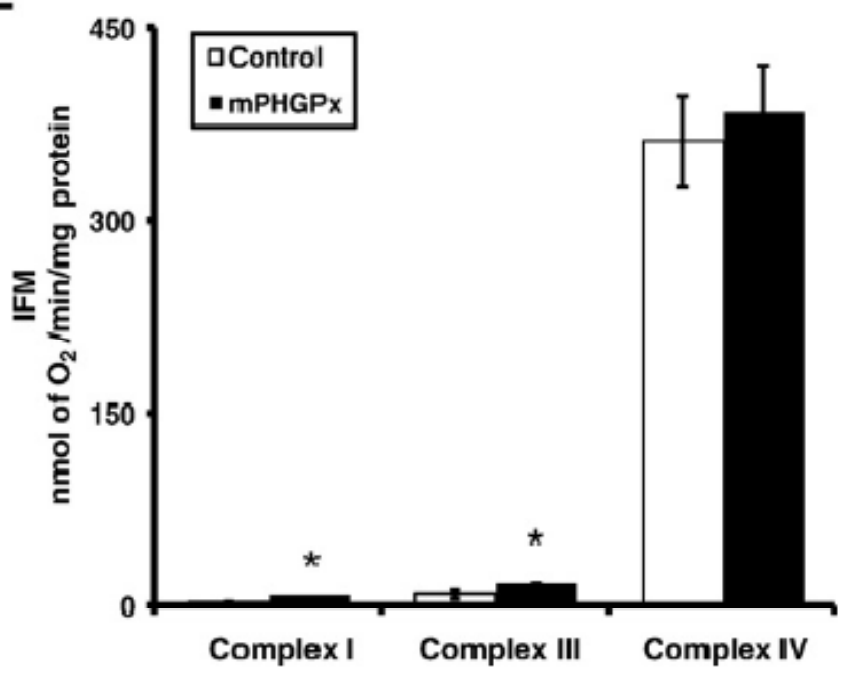


Figure. 5.8. Mitochondria subpopulation electron transport chain (ETC) function following I/R. Individual mitochondrial subpopulations were isolated from $\mathrm{mPHGPx}$ transgenic and littermate control hearts and polarographic measurements performed to index oxygen consumption. Activities of individual ETC complexes were defined as the rate of oxygen consumed in the presence of specific substrates, glutamate/malate

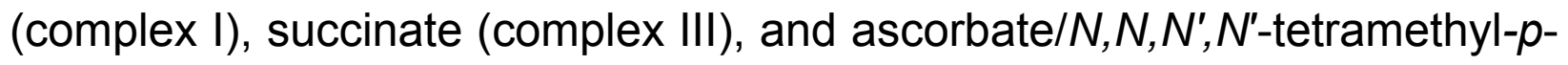
phenylenediamine (complex IV), and were calculated as the fraction that was sensitive to the specific inhibitors rotenone (complex I), antimycin (complex III), and sodium cyanide (complex IV). (A) Representative density plot of isolated mitochondria stained with MitoTracker Deep Red 633, which incorporates into intact mitochondria. Intact mitochondria that take up the MitoTracker Deep Red 633 dye are gated (R1) and represented in blue. Noise and debris, which do not take up the MitoTracker Deep Red 633, are excluded from measurements (outside the R1 gate) and indicated in green. (B) Representative gated density plot indicating size (FSC; forward scatter) vs internal complexity (SSC; side scatter) of isolated SSM. (C) Representative gated density plot indicating size (FSC; forward scatter) vs internal complexity (SSC; side scatter) of isolated IFM. Analyses of forward scatter and side scatter were calculated per 20,000 gated events for all mitochondrial subpopulations. Red hash lines indicate microsphere size references from left to right: $0.5,1,2,4$, and $6 \mu \mathrm{M}$, respectively. (D) SSM ETC complex function following myocardial I/R. (E) IFM ETC complex function following myocardial I/R. Values are expressed as mean $\pm \mathrm{SE}$; $N=6$ for each group. ${ }_{*} P<0.05$ for $\mathrm{mPHGPx}$ vs control. SSM, subsarcolemmal mitochondria; IFM, interfibrillar mitochondria. 
Table 5.1

\section{Cycle Number}

\section{Transgenic Positives}

mPHGPx Line 1

24.3

mPHGPx Line 2

26.0

Hsp27tg

25.4

Transgenic Negatives

mPHGPx Line 1

34.8

mPHGPx Line 2

34.7

Hsp27tg

34.5

Water

34.0 
Table 5.1. MPHGPX Transgenic Mouse Screening. Verification of chimeric transgene presence was accomplished using a real-time PCR approach. Isolated genomic tail DNA was examined by probing for the cytomegalovirus (CMV) immediate early enhancer region indicated in Figure $1 B$, using a custom designed probe. A lower cycle number indicates increasing transgene presence. Two mPHGPx transgenic mouse lines were generated, mPHGPx Line 1 and mPHGPx Line 2 with cycle numbers between 24 and 26. Inclusion of DNA from a known transgenic line (hsp27 tg) developed using the same pCAGGS plasmid is included as a positive internal control, and has a cycle number of approximately 25. Littermate controls for MPHGPx Line 1, mPHGPx Line 2, hsp27 tg, and a water control (no DNA) are included as internal negative controls and display cycle numbers of approximately $34-35$. 


\title{
Chapter 6:
}

\section{Mitochondrial Overexpression of Phospholipid Hydroperoxide Glutathione Peroxidase 4 (mPHGPx) Provides Cardioprotection From Type 1 Diabetes Mellitus Insult}

\author{
Erinne R. Dabkowski ${ }^{1,2}$, Walter A. Baseler ${ }^{1,2}$, Tara L. Croston, ${ }^{2}$, Courtney L. \\ Williamson $^{1,2}$, Matthew J. Powell ${ }^{3}$, Trust T. Razunguzwa ${ }^{3}$, and John M. Hollander ${ }^{1,2}$ \\ ${ }^{1}$ West Virginia University School of Medicine, Division of Exercise Physiology; ${ }^{2}$ Center \\ for Cardiovascular and Respiratory Sciences; Morgantown, WV 26506; ${ }^{3}$ Protea \\ Biosciences Inc.; Morgantown, WV 26507
}

Running Title: MPHGPx and the type 1 diabetic heart 


\section{ABSTRACT}

Mitochondrial dysfunction plays a critical role in the pathogenesis of type 1 diabetes mellitus (DM). Evidence suggests that the inner mitochondria membrane (IMM) and its constituents are specific targets for the deleterious effects associated with type $1 \mathrm{DM}$. As a result, maintenance of IMM integrity represents a specific locus for testing directed prophylactic intervention. We previously reported that type 1 DM imparts differential effects on spatially distinct mitochondrial subpopulations, subsarcolemmal (SSM) and interfibrillar (IFM). The goal of this study was to determine whether overexpression of a mitochondrially-targeted antioxidant enzyme, mitochondria phospholipid hydroperoxide glutathione peroxidase 4 (mPHGPx), which scavenges phospholipids in the IMM, could elicit protection to mitochondria during type 1 diabetic insult, and if the protection was subpopulation-specific. MPHGPx transgenic mice and littermate controls were made diabetic through multiple low dose injections of streptozotocin (STZ). Five weeks following hyperglycemia onset, in vivo analysis of cardiac contractile function revealed significantly decreased ejection fraction and fractional shortening in the diabetic heart $\left({ }^{*} P<0.05\right)$, which was reversed with $\mathrm{mPHGPx}$ overexpression $\left({ }^{*} P<0.05\right)$. MPHGPx overexpression in the diabetic heart, was associated with increased mitochondrial state 3 and state 4 respiration and electron transport chain complex I, III, and IV activities in IFM ( $\left.{ }^{*} P<0.05\right)$, with no differences on SSM. Hydrogen peroxide production and lipid peroxidation were significantly attenuated in the diabetic IFM with overexpression of $\operatorname{mPHGPx}\left({ }^{*} P<0.05\right.$ for both), with no changes in the SSM. An essential IMM component, cardiolipin was preserved in the IFM with ovexpression of $\mathrm{mPHGPx}$ in the diabetic heart. Enhanced post-translational modifications to IMM were also attenuated in the diabetic IFM with overexpressioin of mPHGPx. These results indicate that mPHGPx overexpression provides cardioprotection to the diabetic heart through enhanced mitochondrial function and specifically preservation of IMM. 


\section{INTRODUCTION}

Mitochondrial dysfunction is a major contributor to diabetic cardiomyopathyassociated dysfunction in the type 1 diabetic heart $(7,13,43,46)$. The resultant enhanced reactive oxygen species (ROS) generation and subsequent oxidative damage have been suggested to play a major role in the dysfunction. Elevated glucose and free fatty acid levels present in the diabetic heart have been suggested to drive the formation of ROS $(10,30,51)$. It is believed that initially, increased flux of metabolic substrates generates increased electron leak which leads to enhanced ROS production. The mitochondrion itself is particularly susceptible to oxidative damage because the inner mitochondrial membrane (IMM) is a main site for ROS generation (9). This has implications for IMM integrity in that the IMM is enriched with polyunsaturated fatty acids and oxidizable electron carriers $(12,23)$ that are targets of ROS. Further, many of electron transport chain (ETC) proteins are prone to oxidation because they contain iron-sulphur centers (29), which readily react with ROS and precipitate cellular damage. Enhanced mitochondrial ROS generation has been suggested to directly inhibit ETC complex activities decreasing overall mitochondrial respiration (46). We previously reported that those mitochondria located between the myofibrils, interfibrillar mitochondria (IFM), have enhanced ROS production and subsequent oxidative damage which is associated with diminished overall mitochondrial function in the type 1 diabetic heart (7).

Many proteins and lipids within the IMM are critical targets of elevated ROS production, and their oxidative modification further contributes to mitochondrial dysfunction by limiting sufficient production of ATP. One potential target within the IMM is the mitochondrial-specific phospholipid, cardiolipin. Cardiolipin is a diphosphatidylglycerol enriched in the IMM, containing oxidatively-sensitive acyl groups that may act as selective targets for ROS (19). Cardiolipin plays an essential role in stabilizing protein complexes and translocases within the IMM, facilitating proper mitochondrial function. Cardiolipin directly interacts with a number of mitochondrial proteins within the IMM including $\mathrm{F}_{0} \mathrm{~F}_{1}$-ATPase, ANT, cytochrome $c$, protein import 
machinery, and ETC complexes I, III, and IV $(19,45)$. Cardiolipin is more susceptible to oxidation due to the double bonds located within its fatty acid side chains and it has been suggested that following cardiolipin oxidation, cytochrome $c$ is released which initiates apoptosis (20). Several authors have suggested remodeling and depleted content of cardiolipin in the diabetic heart (15). Previously, we have shown that the most predominant species of cardiolipin is decreased in the IFM suggesting that it is a potential target for damage associated with type 1 diabetes mellitus (7).

Antioxidants play a vital role in defense against enhanced oxidant production in the diabetic heart. Phospholipid hydroperoxide glutathione peroxidase (PHGPx; GPx4), is a monomeric antioxidant enzyme with two distinct forms, a long form (L-form), which contains a mitochondrial targeting sequence and exists in the mitochondrion (mPHGPx), and a short form (S-form), without a mitochondrial targeting sequence which exists in the cytosol $(25,50)$. MPHGPx is unique in that it is the only antioxidant enzyme capable of reducing peroxidized acyl groups in phospholipids, fatty acid hydroperoxides and cholesterol peroxides $(25,48,50)$ in mitochondrial membranes. Since mPHGPx is a lipophilic enzyme, it can interact directly with lipid hydroperoxides within the IMM, and it has been suggested to be the primary antioxidant defense against biomembrane oxidation within the mitochondrion $(20,41)$. Homozygous null GPx4deficient mice die in utero and cell lines derived from GPx4-deficient heterozygous mice are more susceptible to oxidative insult (14). Previous work from our laboratory indicates that transgenic overexpression of $\mathrm{mPHGPx}$ preserves cardiac contractile performance and mitochondrial function while attenuating the accumulation of lipid peroxidation by-products during ischemia/reperfusion insult (8). Nevertheless, to date, no examination has been undertaken to determine whether overexpression of PHGPX provides protection to the diabetic heart. Thus, the goals of the current study were the following; 1) determine whether mPHGPx overexperession preserves cardiac contractile function in the type 1 diabetic heart; 2) determine whether mPHGPx overexperession preserves mitochondrial function in the type 1 diabetic heart; and 3) determine whether mPHGPx overexpression impacts cardiolipin modeling in the type 1 diabetic heart. 


\section{MATERIALS AND METHODS}

\section{Experimental Animals and Diabetes Induction}

The animal experiments in this study conformed to National Institutes of Health (NIH) Guidelines for the Care and Use of Laboratory Animals and were approved by the West Virginia University Animal Care and Use Committee. Mixed sex FVB mice (Charles River Laboratories, Wilmington, MA) were housed in the West Virginia University Health Sciences Center animal facility. Mice were given unlimited access to a rodent diet and water. MPHGPx transgenic mouse lines were generated as previously described $(16,18,26)$. Briefly, the pCAGGS vector places the mPHGPx gene under the control of the human cytomegalovirus (CMV). All littermate control and transgenic mice were generated using an FVB background, and experimental procedures were performed on animals of approximately 8 weeks of age. Type 1 diabetes mellitus was induced in 8-wk-old control and transgenic (mPHGPx) mice following the protocol of the Animal Models of Diabetic Complications Consortium using multiple low-dose streptozotocin (STZ; Sigma, St. Louis, MO) injections. Injections of $50 \mathrm{mg} / \mathrm{kg}$ body wt STZ dissolved in sodium citrate buffer $(\mathrm{pH} 4.5)$ were performed daily for 5 consecutive days after 6 hours of fasting. Mice that served as vehicle controls were given the same volume per body weight of sodium citrate buffer. One week postinjection, hyperglycemia was confirmed by measuring urinary glucose (Chemstrip 2GP Urine test strips, Roche Diagnostics, Indianapolis, IN), where $>2,000 \mathrm{mg} / \mathrm{dl}$ was considered diabetic. Five weeks post hyperglycemia onset, animals were killed for further experimentation.

\section{Echocardiographic Assesment}

Transthoracic echocardiography was performed in conscious mice following the protocols provided by the Animal Models of Diabetic Complications Consortium (AMDCC; www.amdcc.org). Briefly, Left ventricular (LV) fractional shortening (\%FS) and ejection fraction (\%) were assessed in all mice with echocardiography which was 
equipped with a 13-MHz linear probe (Vivid i; GE Medical Systems, Milwaukee, Wisconsin, USA). With mice in the left lateral decubitus position, a parasternal short-axis view was obtained for LV M-mode imaging at the papillary muscle level and LV chamber dimensions were determined. LV fractional shortening and LV ejection fraction are calculated from the M-mode measurements.

\section{Preparation of Individual Mitochondrial Subpopulations}

At 5 weeks post hyperglycemia onset, control, diabetic, mPHGPx and mPHGPx diabetic were sacrificed, and the hearts were excised. Hearts were rinsed in phosphate buffered saline (PBS, pH 7.4), then blotted dry and weighed. SSM and IFM were isolated as previously described following the methods of Palmer et al. (33) with minor modifications $(6-8,52)$. Protein concentrations were determined using the Bradford method with bovine serum albumin (BSA) as a standard (1).

\section{Mitochondria Size and Internal Complexity}

To asses mitochondrial subpopulation size and complexity, we performed flow cytometry analyses using a FACS Calibur equipped with a 15 MW 488-nm argon laser and 633 red diode laser (Becton Dickinson, San Jose, CA) as previously described (6-8, 52). All flow cytometric measurements were performed under the supervision of the West Virginia University Flow Cytometry Core Facility.

\section{Electron Transport Chain (ETC) Respiration}

State 3 and state 4 respiration was assessed in isolated mitochondrial subpopulations as previously described $(2,3,17)$ with slight modifications (8). Isolated mitochondrial subpopulations were resuspended in respiration buffer containing (in $\mathrm{mmol} / \mathrm{L}: 80 \mathrm{KCl}, 50 \mathrm{MOPS}, 1 \mathrm{EGTA}, 5 \mathrm{KH} 2 \mathrm{PO} 4$, and $1 \mathrm{mg} / \mathrm{ml} \mathrm{BSA}$ ) and equal volumes 
were loaded into a Gilson chamber (Gilson, Middleton, WI) attached to a Yellow Springs Instruments 5300 biological oxygen monitor (YSI, Yellow Springs, OH). The substrates glutamate $(5 \mathrm{mM})+$ malate $(5 \mathrm{mM})$ were used to initiate respiration and measurement of state $3(1 \mathrm{mM}$ ADP) and state 4 (ADP-limited) respiration were made as previously described (6-8).

\section{Electron Transport Chain (ETC) Complex Activities}

ETC activities of complexes I, III and IV were measured spectrophotometrically as previously described $(6-8,49)$. Briefly, complex I activity was determined by measuring the oxidation of NADH at $340 \mathrm{~nm}$, complex III activity was determined by following the reduction of cytochrome $c$ at $550 \mathrm{~nm}$ in the presence of reduced decylubiquinone $(50 \mu \mathrm{M})$, and complex IV activity was determined by measuring the oxidation of cytochrome $c$ at $550 \mathrm{~nm}$. Protein content was determined as described above (1), and values expressed as activities in nanomoles substrate consumed per minute per milligram of protein.

\section{ATP Synthase Activity}

ATP synthase activity was measured in mitochondrial subpopulations as oligomycin-sensitive ATPase activity using an assay coupled with pyruvate kinase which converts the ADP to ATP and produces pyruvate from phosphoenolpyruvate as previously described. $(6,11,39,44)$. Protein content was assessed as above (1) with final values expressed as nanomoles consumed per minute per milligram of protein, which was equal to the nanomoles of $\mathrm{NADH}$ oxidized per minute per milligram of protein. 


\section{Lipid Peroxidation Products}

Lipid peroxidation by-products malondialdehyde (MDA) and 4-hydroxyalkenal (4$\mathrm{HAE}$ ), stable end products formed from the oxidation of polyunsaturated fatty acids and esters, were assessed as previously described (6-8). Absorbance was measured on a Molecular Devices Flex Station 3 spectrophotometer (Molecular Devices, Sunnyvale, CA) and protein content was assessed as described above (1) with final values expressed per milligram of protein.

\section{Mitochondrial Membrane Potential ( $\left.\Delta \Psi_{m}\right)$}

Mitochondrial membrane potential $\left(\Delta \Psi_{\mathrm{m}}\right)$ was measured on freshly isolated mitochondria by flow cytometry using the ratiometric dye 5,5',6,6'-tetrachloro-1,1',3,3'tetraethylbenzimidazol carbocyanine iodide (JC-1) (Molecular Probes, Carlsbad, CA) as previously described $(6,52)$. Addition of $200 \mu \mathrm{M}$ of dinitrophenol (DNP), which dissipates $\Delta \Psi \mathrm{m}$, was used as an assay control.

\section{Cardiolipin}

Mitochondrial subpopulations were isolated from control, mPHGPx, diabetic and mPHGPx diabetic hearts. An $n=4$ for each of the four groups was pooled and sent off to Avanti Polar Lipids (Alabaster, $\mathrm{AL}$ ) for cardiolipin analysis. Extraction and analysis of total cardiolipin species was based on the protocols of Sparagna et al. (47) and Minkler et al. (27).

\section{Hydrogen Peroxide $\left(\mathrm{H}_{2} \mathrm{O}_{2}\right)$ Production}

$\mathrm{H}_{2} \mathrm{O}_{2}$ production in isolated mitochondrial subpopulations was determined using the oxidation of the fluorogenic indicator amplex red in the presence of horseradish 
peroxidase (54). The concentrations of horseradish peroxidase and amplex red in the incubation were $0.1 \mathrm{unit} / \mathrm{ml}$ and $50 \mu \mathrm{M}$ and detection of fluorescence was assessed on a Molecular Devices Flex Station 3 fluorescence plate reader (Molecular Devices, Sunnyvale, CA) with $530 \mathrm{~nm}$ excitation and $590 \mathrm{~nm}$ emission wavelengths. Standard curves were obtained by adding known amounts of $\mathrm{H}_{2} \mathrm{O}_{2}$ to the assay medium in the presence of the substrates amplex red and horseradish peroxidase. Final values were calculated and presented as pmol/mg of protein. $\mathrm{H}_{2} \mathrm{O}_{2}$ production was initiated in mitochondria using glutamate $(5.0 \mathrm{mM}) /$ malate $(5.0 \mathrm{mM})$ as substrates. The addition of catalase diminished fluorescence confirming dissipation of $\mathrm{H}_{2} \mathrm{O}_{2}$ presence in the sample.

\section{Multi Dimensional Protein Identificiation Technology (MudPIT)}

Pooled IFM subpopulations $(n=4)$ from control, diabetic, and mPHGPx diabetic hearts were analyzed for post-translational modifications as previously described (6). Briefly, samples were lysed and precipitated overnight in acetone at $-20^{\circ} \mathrm{C}$ and pellets were resuspended in $20 \mu \mathrm{L}$ of $0.5 \mathrm{M}$ triethylammonium bicarbonate (TEAB; $\mathrm{pH}$ 8.5). Protein contents were determined using a 2-D Quant Kit (Amersham, Piscataway, NJ) and pooled samples were than denatured with $0.1 \%$ SDS and reduced with $5 \mathrm{mM}$ tris-(2carboxyethyl) phosphine (TCEP). After incubation for 1 hour at $60^{\circ} \mathrm{C}$, cysteines were blocked with $10 \mathrm{mM}$ methyl methane thiosulfonate (MMTS) in isopropanol, and the samples were incubated at room temperature for 10 minutes. $10 \mu \mathrm{L}$ of sequencing grade trypsin (Applied Biosystems, Foster City, CA) was added in a trypsin/protein ratio of $1: 20$, and the samples incubated at $37^{\circ} \mathrm{C}$ overnight. After digestion, the ultracomplex protein digests were subject to Multi Dimensional Protein Identificiation Technology (MudPIT) analysis (24). After lyophilization, the digest mixture was reconstituted in strong cation exchange (SCX) loading buffer ( $5 \mathrm{mM}$ ammonium formate in $20 \%$ acetonitrile; pH 3.0 ) to be fractionated with SCX SpinTips (Protea Biosciences, Morgantown, WV) per the manufacturer's protocol. Briefly, the sample solution was loaded centrifugally onto the SCX SpinTip. The non-adsorbing solution that passed 
through the SCX SpinTip was collected. Eight different elution solutions were used to fractionate the peptides $(20,60,100,150,200,250,400$, and $500 \mathrm{mM}$ ammonium formate in $10 \%$ acetonitrile) in a step-wise manner, for a total of nine sample fractions. The collected fractions were cleaned by repeated lyophilization and reconstituted in a $0.1 \mathrm{M}$ acetic acid solution, and then lyophilized to dryness. The fractions were then submitted for LC-MALDI TOF/TOF mass spectral analysis for identification of proteins and post-translational modifications that are present.

\section{Mass Spectrometry (MS) Analyses}

The LC-MALDI mass spectrometry system utilized was an ABI Tempo LC MALDI spotter with Tempo LC MALDI v.2.00.09 data acquisition and processing software. Lyophilized SCX sample fractions were reconstituted in LC aqueous run buffer $(0.1 \%$ trifluoroacetic acid, $2 \%$ acetonitrile) and was injected onto a Zorbax $\mathrm{C}_{18}$ chromatographic column, $150 \times 0.3 \mathrm{~mm}$ (Agilent Technologies, Wilmington, DE). The peptides were eluted from the column using an acetonitrile/trifluoroacetic acid gradient (2-72\% acetonitrile in 35 minutes) and spotted directly onto a MALDI plate in 6 second spot fractions. The MALDI spots were analyzed using an ABI 4800 MALDI TOF/TOF analyzer operated with 4000 Series Explorer software. The MS acquisition was in positive ion reflector mode with 400 laser shots per spectrum performed. The 15 strongest precursors per spot were chosen for MS/MS and the MALDI spot was interrogated until at least 4 peaks in the MS/MS, spectra achieved a $S / N \geq 70$.

The resulting MS/MS spectra were analyzed using $A B I$ Protein ProteinPilot software 2.0 (Applied Biosystems, Foster City, CA). The spectral data was searched against the mouse protein database (NCBI nr.fasta database customized to select for all mouse proteins) for identification of the peptides and corresponding proteins. After database correlation analysis, the proteins were grouped and scored. The Pro Group algorithm of ProteinPilot generated a ProtScore that is a cumulative score from each of the peptides used by the algorithm in the protein identification. Protein scores (ProtScore) above 2.0, 1.0, and 0.47 expressed the percent confidence levels of $>99$, 
$>90$, and $>66 \%$, respectively. Each peptide match showed post-translational modifications present as mass spectral shifts identified during the database correlation analysis.

\section{Statistics}

Means and standard errors (SEM) were calculated for all data sets. Data were analyzed with a two-way analysis of variance (ANOVA) method to evaluate the main treatment effects, diabetes induction and mPHGPx transgene presence (GraphPad Software Inc., La Jolla, CA). Fisher's Least Significant Difference (LSD) post hoc tests were performed to determine the significant differences among means. When appropriate a Student's T-test was employed. ${ }^{*} P<0.05$ was considered significant. 


\section{RESULTS}

\section{Cardiac Contractile Function}

Cardiac contractile function was significantly altered in the diabetic mice as compared to control. Ejection fraction and fractional shortening were significantly decreased in the diabetic heart compared to control. With overexpression of mPHGPx in the diabetic heart, ejection fraction and fractional shortening were restored back to control values (Table 6.1, ${ }^{*} P<0.05$ ).

\section{Mitochondrial Subpopulation Morphology}

Forward scatter (FSC), a measurement of size, was significantly decreased in the diabetic IFM as compared to control and restored back to control values with overexpression of mPHGPx (Figures $6.1 \mathrm{~F},{ }^{*} P<0.05$ ), yet no significant changes were observed in SSM (Figures 6.1D). Mitochondrial internal complexity (SSC) was significantly decreased in only the diabetic IFM as compared to control IFM (Figure $6.1 \mathrm{G},{ }^{*} P<0.05$ ) with no significant changes in the SSM (Figure 5.1E). Though it was not statistically significant, there was an increase in complexity with over expression of mPHGPx in the diabetic IFM (Figure 6.1G).

\section{Mitochondrial Subpopulation Functional Assessment}

Assessment of state 3 and state 4 respiration with the substrates glutamate/malate revealed a significant decrease in state 3 and state 4 respiration in diabetic IFM as compared to Control IFM (Figure 6.2B; ${ }^{*} P<0.05$ ), with no significant changes between control and diabetic SSM (Figure 6.2A). Further, overexpression of mPHGPx in the diabetic heart restored state 3 and state 4 respiration in the IFM with no significant effects observed in the SSM (Figure 6.2B). ETC complexes I, III and IV activities were also significantly decreased in diabetic IFM as compared to control IFM 
(Table 6.2; ${ }^{*} P<0.05$ ), with no significant differences between control SSM and diabetic SSM (Table 6.2). Overexpression of MPHGPx in the diabetic heart enhanced ETC complex I,III and IV activities specifically in the IFM back to control IFM values (Table 6.2; $\left.{ }^{*} P<0.05\right)$.

\section{ATP Synthase Activity}

Alterations to ETC function can contribute to altered oxidative phosphorylation. ATP synthase activity was significantly decreased in the diabetic IFM as compared to control IFM (Figure 6.3B; ${ }^{*} P<0.05$ ), with no significant differences observed between the diabetic SSM and control SSM (Figure 6.3A). Overexpression of mPHGPx enhanced ATP synthase activity in the diabetic IFM back to control IFM values (Figure 6.3B, ${ }^{*} P<0.05$ ), with no significant effects on any of the SSM groups (Figure 6.3A).

\section{Hydrogen Peroxide Production}

Hydrogen peroxide can directly be scavenged by mPHGPx. Glutamate/malate driven hydrogen peroxide production was significantly increased in the diabetic IFM as compared to control IFM (Figure 6.4B, ${ }^{*} P<0.05$ ), with no significant changes in diabetic SSM compared to control IFM (Figure 6.4A). Overexpression of mPHGPx in the diabetic heart attenuated $\mathrm{H}_{2} \mathrm{O}_{2}$ production in the IFM $\left(6.4 \mathrm{~B}\right.$, $\left.{ }^{*} \mathrm{P}<0.05\right)$, with no significant changes in the SSM.

\section{Post-translational modifications}

Table 5.3 represents protein post-translational modifications no longer present in the diabetic IFM with overexpression of mPHGPx. Overexpression of mPHGPx decreased PTMs identified on 68 peptide sequences of 44 different proteins in the diabetic IFM (Table 6.3). Post-translational modifications that were attenuated include 
oxidations, deamidations and acetylations. Interestingly, 5 IMM proteins and 4 matrix proteins identified no longer contained oxidative modifications with overexpression of mPHGPx in the diabetic IFM. Of the 5 IMM proteins, 3 of these proteins were ETC proteins that play important roles in oxidative phosphorylation. Matrix proteins that were no longer oxidized with overexpression of $\mathrm{MPHGPx}$ included 2 TCA cycle and 2 FAO proteins, also proteins that ultimately contribute to proper ATP production. Reduction of deamidations to proteins was even more prevalent than reduction of oxidations with overexpression of mPHGPx. $16 \mathrm{IMM}$ proteins and 21 matrix associated proteins identified no longer contained deamidations with overexpression of $\mathrm{mPHPGx}$ in the diabetic IFM.

\section{Lipid Peroxidation}

Lipid peroxidation by-products 4-HAE and MDA were significantly increased in the diabetic IFM as compared to control IFM, (Figure 6.5B, ${ }^{*} P<0.05$ ), with no significant changes in diabetic SSM compared to control SSM (Figure 6.5A, ${ }^{*} P<0.05$ ). Further, MPHGPx overexpression specifically decreased accumulation of lipid peroxidation byproducts in the diabetic IFM back to values similar to that of control IFM (Figure 6.5B, $\left.{ }^{*} P<0.05\right)$, suggesting the ability of $\mathrm{mPHGPx}$ to attenuate ROS induced damage to lipids within the IFM.

\section{Cardiolipin}

Cardiolipin is a phospholipid within the IMM that plays a critical role in proper mitochondrial function. The most predominant species of cardiolipin ( $/ \mathrm{z} / \mathrm{1448})$ and total cardiolipin content were decreased in the diabetic IFM compared to control IFM with no changes between diabetic SSM and control SSM. The oxidized species of Cardiolipin, m/z 1496, was increased in the diabetic IFM as compared to control IFM with no differences observed between control and diabetic SSM. Further, overexpression of $\mathrm{mPHGPx}$ in the diabetic heart increased the most predominant 
species of cardiolipin and total cardiolipin content in the IFM with no changes in the SSM (Table 6.4). The oxidized form of cardiolipin was attenuated with overexpression of $\mathrm{mPHGPx}$ in the diabetic IFM with no effect on the SSM. The overall ratio of nonoxidized to oxidized was higher with overexpression of MPHPGx in the diabetic IFM with no changes in the SSM (Table 6.4).

\section{Inner Mitochondrial Membrane Potential $\left(\Delta \Psi_{m}\right)$}

Inner mitochondrial membrane potential, the ratio of orange to green fluorescence, was significantly decreased in the diabetic IFM as compared to control IFM (Figure 6.6B, ${ }^{*} P<0.05$ ), with no changes in diabetic SSM as compared to control SSM (Figure 6.6A). Overexpression of $\mathrm{mPHGPx}$ enhanced $\Delta \Psi_{\mathrm{m}}$ in the diabetic IFM back to control IFM values (Figure $6.6 \mathrm{~B},{ }^{*} \mathrm{P}<0.05$ ), with no significant differences observed in SSM (Figure 6.6A). 


\section{DISCUSSION}

Mitochondrial dysfunction has been suggested to play a critical role in the pathogenesis of diabetes mellitus. Enhanced oxidative stress and oxidative damage potentiates this dysfunction. Mitochondria are particularly susceptible to oxidative damage because the IMM is the main site of ROS generation and contains components that are highly susceptible to oxidation such as polyunsaturated fatty acids (PUFAS) and oxidizable electron carriers $(12,23)$. Enhanced mitochondrial ROS generation has been suggested to disrupt integral components of the IMM such as ETC complexes decreasing overall mitochondrial function (46). Previously, we have published suggesting that the IFM have enhanced ROS production and increased oxidative damage to proteins and lipids within the IMM which was associated with diminished mitochondrial function (7). One potential target within the IMM with type 1 diabetes mellitus is the mitochondrial-specific phospholipid, cardiolipin. Cardiolipin plays an essential role of stabilizing protein complexes and translocases within the IMM, facilitating proper mitochondrial function $(4,19)$. Many proteins and lipids within the IMM are targets of elevated ROS production, and their oxidative modification may contribute to mitochondrial deficiencies. One antioxidant enzyme within the mitochondria that plays a critical role in attenuating ROS induced damage to the IMM is mPHGPx. MPHGPx specifically associates with the IMM and scavenges phospholipid hydroperoxides including cardiolipin hydroperoxide in an attempt to preserve the IMM integrity and function $(20,50)$. As a result, the current study was designed to test the hypothesis that mitochondria-specific overexpression of PHGPx could attenuate ROSinduced damage to the mitochondria by preservation of the IMM structure and function which in turn, improves cardiac contractile function in the diabetic heart. In attempts to delineate the therapeutic potential of overexpression of mPHGPx, we utilized our previously generated transgenic mouse line that overexpresses mPHGPx (8).

Previously, we have published suggesting that transgenic overexpression of mPHGPx preserved overall cardiac contractile function with $\mathrm{I} / \mathrm{R}$ (8). MPHGPX overexpression also improved mitochondrial function and attenuated oxidative damage 
suggesting its potential as a therapeutic in alleviating ROS mediated dysfunction. To determine whether overexpression of $\mathrm{MPHGPx}$ influences diabetic-associated cardiac dysfunction, we examined contractile parameters in the STZ-induced type 1 diabetic heart. Cardiac abnormalities, such as decreased ejection fraction and fractional shortening, are often present in STZ-induced type 1 diabetic heart. Cardiac dysfunction in the diabetic heart can be attributed to mitochondrial dysfunction through altered calcium dynamics (31), enhanced oxidative stress (46) and defects in oxidative phosphorylation (13). In the present study, the decreased ejection fraction and fractional shortening observed in the diabetic heart was reversed with overexpression of mPHGPx (Table 6.1). Though the direct link is not entirely clear, it is plausible that improved mitochondrial function through attenuation of ROS-induced damage may contribute to enhanced ATP production and improved calcium regulation by the mitochondria. To better understand the contribution of mPHGPx to enhanced contractile function in the diabetic heart, we then examined the impact of mPHPGx on morphological, functional and biochemical characteristics of the subpopulations of mitochondria in the diabetic heart with overexpression of mPHGPx.

Mitochondrial morphological manifestations have been observed in the diabetic state $(7,22)$. Consistent with our previous findings $(7)$, the diabetic IFM were smaller and less complex with no changes in the SSM (Figure 6.1F and 6.G). Overexpression of $\mathrm{mPHGPx}$ restored mitochondrial size and internal complexity in the diabetic IFM. It is possible that because mPHGPx can directly interact with phospholipids within the IMM, that preservation of these phospholipids may contribute to maintenance of mitochondrial morphology. Due to the role played by mPHGPx in scavenging phospholipid hydroperoxides and preserving IMM integrity, we explored whether mPHGPx overexpression would be protective to ETC complexes. ETC complexes are surrounded by phospholipids in the IMM that contribute to their stability, integrity, and function. Many of these phospholipids are more easily prone to oxidation which in turn can damage the ETC (32). The diabetic IFM had decreased overall state 3 and state 4 respiration (Figure 6.2B) as well as diminished ETC activity at complexes I,III and IV. These results are in agreement with our previous study indicating diminished ETC function specific to the IFM. Overexpression of mPHGPx improved mitochondrial 
function through enhanced overall state 3/state 4 respiration and increased ETC activities in the diabetic IFM (Figure 6.2B). Alterations in mitochondrial ETC function may result in the inability of the mitochondria to adequately provide ATP. Therefore, we examined ATP synthase activity in the diabetic heart. Overall, there was a significant decrease in ATP synthase activity in the diabetic IFM (Figure 6.3B) which was reversed with overexpression of mPHGPx. These data suggest that mPHGPx provides protection to the mitochondria by improving ETC function and subsequent ATP synthesis. It is plausible that mPHGPx can attenuate ROS-induced damage to the IMM thus preserving IMM constituents, such as oxidative phosporylation machinery, allowing for proper mitochondrial function.

The IMM is the main site of ROS formation therefore making the mitochondrion more susceptible to ROS-induced damage $(9,40)$. Previously, we have observed enhanced superoxide production with glutamate and malate as substrates in the diabetic IFM (7). Superoxide is dismutated into hydrogen peroxide which can react readily with iron producing the highly reactive hydroxyl radical. MPHGPx has the ability to directly scavenge mitochondrial hydrogen peroxide thus presumably decreasing hydroxyl radical formation. In the present study, enhanced hydrogen peroxide formation was found in the diabetic IFM (Figure 6.4B) which corresponds to our previous findings suggesting enhanced ROS specifically in the diabetic IFM (7). Interestingly, excess hydrogen peroxide formation was attenuated in the diabetic heart with overexpression of mPHGPx (Figure 6.4B). Unlike other antioxidants, mPHGPx is a unique enzyme that it is associated with subcellular membranes and has been suggested to be the primary enzymatic defense against oxidation of phospholipids in biomembranes (50). Because reactive products derived from the oxidation of lipids promote deleterious effects on membrane constituents, we examined MDA and 4-HAE content in isolated mitochondria in the diabetic heart. Similar to our previous findings, MDA and 4-HAE were increased in the diabetic IFM (Figure 6.5B) with no changes in the SSM (Figure 6.5A). This effect was attenuated with overexpression of mPHGPx in the diabetic IFM, as compared to controls (Figure 6.5B), suggesting the ability of mPHPGx to directly reduce oxidative damage to lipids within the diabetic IFM. 
One phospholipid predominantly within the IMM that is absolutely essential for proper mitochondrial function through a variety of mechanisms is cardiolipin. Cardiolipin in necessary for the formation of contact sites between inner and outer membranes, stabilization of respiratory chain complexes and is involved in mitochondrial apoptotic signaling $(19,53)$. Cardiolipin modulation has been observed in a number of pathologies including ischemia/reperfusion, aging and diabetes mellitus (4, 15, 35-38). In the present study, multiple cardiolipin species were examined and were changing in the diabetic heart (Table 6.4). In agreement with our previous study (7), the most predominant species of cardiolipin (T18:2) at a m/z of 1448 was decreased in the diabetic IFM with no changes in the SSM (Table 6.4). Overexpression of mPHGPx in the diabetic heart increased T18:2 cardiolipin specifically in the IFM beyond control values suggesting the ability of mPHPGx to preserve cardiolipin. This notion has been previously suggested (28) due to the ability of PHGPx to directly interact with biological membranes. Further, cardiolipin depletion has been suggested to decrease membrane potential disrupting the mitochondrial proton gradient and the ability to generate ATP. Analysis of membrane potential in the present study revealed decreases in delta psi in the diabetic IFM (Figure 6.6B) which was reversed with overexpression of $\mathrm{mPHGPx}$ (Figure 6.6B). The abundance of unsaturated double bonds within cardiolipin's structure makes it more susceptible oxidative modification. Oxidative damage to cardiolipin would be detrimental and impact the biochemical function of the IMM such as altered membrane fluidity, ion permeability, surface charge, passive electric properties, membranous enzyme activity and could even initiate apoptosis (34). Interestingly, the oxidized form of cardiolipin, present at a $\mathrm{m} / \mathrm{z}$ ratio of 1496 , was increased in the diabetic IFM (Table 6.4). Overexpression of mPHGPx decreased the content of oxidized cardiolipin which inturn, increased the ratio of non-oxidized to oxidized cardiolipin in the diabetic IFM. These data suggest that MPHGPx is capable of sustaining cardiolipin content and attenuated oxidative damage to cardiolipin in the diabetic IFM. Because cardiolipin is necessary for IMM function and integrity, preservation of cardiolipin may be a potential mechanism in which $\mathrm{mPHGPx}$ provides protection in the diabetic heart.

An oxidative environment can often lead to enhanced post-translational modification (PTM) to proteins including oxidations and deamidations (5). Though the 
contribution of deamidation and oxidation to protein dysfunction is somewhat unclear, these PTMs may alter protein structure/function making the protein more susceptible for degradation by the proteasome system $(21,42)$. In the present study, overexpression of mPHGPx decreased PTMs identified on 68 peptide sequences of 44 different proteins in the diabetic IFM (Table 6.3). Interestingly, majority of the proteins that no longer contained oxidative modifications with overexpression of mPHGPx were IMM proteins. Of the $5 \mathrm{IMM}$ proteins, 3 of these proteins were ETC proteins that play important roles in oxidative phosphorylation. Oxidative conditions have also been considered as a way through which proteins become more susceptible to deamidation. Though the underlying mechanism is not clear, the evidence suggests that oxidative conditions may induce an increased flexibility of the polypeptide backbone or a transient unfolding of the proteins, allowing for deamidation to occur (5). Moreover, UV irradiation, which causes increased ROS formation, leads to an increased protein deamdation in cultured cells (5). In the present study, reduction of deamidations to proteins was even more prevalent than reduction of oxidations with overexpression of mPHGPx (Table 6.3). 16 IMM proteins and 21 matrix associated proteins identified no longer contained deamidations with overexpession of $\mathrm{MPHPGx}$ in the diabetic IFM. Many of these proteins are also involved in oxidative phosphorylation. Attenuation of PTMs of IMM proteins with overexpression of mPHGPx in the diabetic heart may contribute to improved functionality of proteins critical for proper oxidative phosphorylation and adequate ATP production.

In conclusion, we report for the first time, that mitochondria-specific transgenic overexpression of PHGPx in a mouse model provides cardioprotection through enhanced contractile function in the type 1 diabetic heart. Further, cardioprotection is associated with enhanced IMM function and preservation of a key IMM constituent, cardiolipin. These findings provide further rationale for the use of mPHGPx as a potential mitochondrially-targeted therapeutic that is capable of providing protection in the diabetic heart. 


\section{ACKNOWLEDGEMENTS}

We would like to thank Dr. Christopher Cuff and the contributions from the West Virginia University Flow Cytometry Core Facility. We would also like to thank Dr. Peter Mathers and the contributions from the West Virginia University Transgenic Animal Core Facility. 


\section{References}

1. Bradford MM. A rapid and sensitive method for the quantitation of microgram quantities of protein utilizing the principle of protein-dye binding. Analytical biochemistry 72: 248-254, 1976.

2. Chance B and Williams GR. Respiratory enzymes in oxidative phosphorylation. I. Kinetics of oxygen utilization. J Biol Chem 217: 383-393, 1955.

3. Chance B and Williams GR. Respiratory enzymes in oxidative phosphorylation. VI. The effects of adenosine diphosphate on azide-treated mitochondria. J Biol Chem 221: 477-489, 1956.

4. Chicco AJ and Sparagna GC. Role of cardiolipin alterations in mitochondrial dysfunction and disease. Am J Physiol Cell Physiol 292: C33-44, 2007.

5. Cimmino A, Capasso R, Muller F, Sambri I, Masella L, Raimo M, De Bonis ML, D'Angelo S, Zappia V, Galletti P, and Ingrosso D. Protein isoaspartate methyltransferase prevents apoptosis induced by oxidative stress in endothelial cells: role of $\mathrm{Bcl}-\mathrm{XI}$ deamidation and methylation. PLoS One 3: e3258, 2008.

6. Dabkowski ER, Baseler WA, Williamson CL, Powell M, Razunguzwa TT, Frisbee JC, and Hollander JM. Mitochondrial Dysfunction in the Type 2 Diabetic Heart is Associated with Alterations in Spatially-Distinct Mitochondrial Proteomes. Am J Physiol Heart Circ Physiol, 2010.

7. Dabkowski ER, Williamson CL, Bukowski VC, Chapman RS, Leonard SS, Peer CJ, Callery PS, and Hollander JM. Diabetic cardiomyopathy-associated dysfunction in spatially distinct mitochondrial subpopulations. Am J Physiol Heart Circ Physiol 296: H359-369, 2009.

8. Dabkowski ER, Williamson CL, and Hollander JM. Mitochondria-specific transgenic overexpression of phospholipid hydroperoxide glutathione peroxidase 
(GPx4) attenuates ischemia/reperfusion-associated cardiac dysfunction. Free Radic Biol Med 45: 855-865, 2008.

9. Droge W. Free radicals in the physiological control of cell function. Physiol Rev 82: 47-95, 2002.

10. Du Y, Miller CM, and Kern TS. Hyperglycemia increases mitochondrial superoxide in retina and retinal cells. Free Radic Biol Med 35: 1491-1499, 2003.

11. Feniouk BA, Suzuki T, and Yoshida M. Regulatory interplay between proton motive force, ADP, phosphate, and subunit epsilon in bacterial ATP synthase. J Biol Chem 282: 764-772, 2007.

12. Ferrari R, Ceconi C, Curello S, Cargnoni A, De Giuli F, and Visioli 0 . Occurrence of oxidative stress during myocardial reperfusion. Molecular and cellular biochemistry 111: 61-69, 1992.

13. Flarsheim CE, Grupp IL, and Matlib MA. Mitochondrial dysfunction accompanies diastolic dysfunction in diabetic rat heart. Am J Physiol 271: H192-202, 1996.

14. Garry MR, Kavanagh TJ, Faustman EM, Sidhu JS, Liao R, Ware C, Vliet PA, and Deeb SS. Sensitivity of mouse lung fibroblasts heterozygous for GPx4 to oxidative stress. Free Radic Biol Med 44: 1075-1087, 2008.

15. Han X, Yang J, Yang K, Zhao Z, Abendschein DR, and Gross RW. Alterations in myocardial cardiolipin content and composition occur at the very earliest stages of diabetes: a shotgun lipidomics study. Biochemistry 46: 6417-6428, 2007.

16. He H, Giordano FJ, Hilal-Dandan R, Choi D, Rockman HA, McDonough PM, Bluhm WF, Meyer M, Sayen MR, Swanson E, and Dillmann WH. Overexpression of the rat sarcoplasmic reticulum $\mathrm{Ca}^{2+}$ ATPase gene in the heart of transgenic mice accelerates calcium transients and cardiac relaxation. J Clin Invest 100: 380-389, 1997.

17. Hofhaus G, Shakeley RM, and Attardi G. Use of polarography to detect respiration defects in cell cultures. Methods in enzymology 264: 476-483, 1996.

18. Hollander JM, Martin JL, Belke DD, Scott BT, Swanson E, Krishnamoorthy V, and Dillmann WH. Overexpression of wild-type heat shock protein 27 and a nonphosphorylatable heat shock protein 27 mutant protects against 
ischemia/reperfusion injury in a transgenic mouse model. Circulation 110: 3544-3552, 2004.

19. Houtkooper RH and Vaz FM. Cardiolipin, the heart of mitochondrial metabolism. Cell Mol Life Sci 65: 2493-2506, 2008.

20. Imai $\mathbf{H}$ and Nakagawa $\mathbf{Y}$. Biological significance of phospholipid hydroperoxide glutathione peroxidase (PHGPx, GPx4) in mammalian cells. Free radical biology \& medicine 34: 145-169, 2003.

21. Iwai K, Drake SK, Wehr NB, Weissman AM, LaVaute T, Minato N, Klausner RD, Levine RL, and Rouault TA. Iron-dependent oxidation, ubiquitination, and degradation of iron regulatory protein 2: implications for degradation of oxidized proteins. Proc Natl Acad Sci U S A 95: 4924-4928, 1998.

22. Kelley DE, He J, Menshikova EV, and Ritov VB. Dysfunction of mitochondria in human skeletal muscle in type 2 diabetes. Diabetes 51: 2944-2950, 2002.

23. Lashin $\mathbf{O}$ and Romani A. Mitochondria respiration and susceptibility to ischemia-reperfusion injury in diabetic hearts. Archives of biochemistry and biophysics 420: 298-304, 2003.

24. Lin D. Multidimensional Protein Identification Technology as an Effective Tool for Proteomics. American Genomic/Proteomic Technology 1: 38-46, 2001.

25. Lu J and Holmgren A. Selenoproteins. J Biol Chem 284: 723-727, 2009.

26. Marber MS, Mestrill R, Chi S, Sayen RS, Yellon DM, and Dillmann WH. Overexpression of the rat inducible $70-\mathrm{kD}$ heat stress protein in a transgenic mouse increases the resistance of the heart to ischemic injury. J Clin Invest 95: 1446-1456, 1995.

27. Minkler PE and Hoppel CL. Separation and characterization of cardiolipin molecular species by reverse-phase ion pair high-performance liquid chromatographymass spectrometry. J Lipid Res 51: 856-865, 2010.

28. Nakagawa Y. Role of mitochondrial phospholipid hydroperoxide glutathione peroxidase (PHGPx) as an antiapoptotic factor. Biol Pharm Bull 27: 956-960, 2004.

29. Newsholme E and AR L. Biochemistry for the medical sciences. Chichester: John Wiley \& Sons, 1983. 
30. Nishikawa T, Edelstein D, Du XL, Yamagishi S, Matsumura T, Kaneda Y, Yorek MA, Beebe D, Oates PJ, Hammes HP, Giardino I, and Brownlee M. Normalizing mitochondrial superoxide production blocks three pathways of hyperglycaemic damage. Nature 404: 787-790, 2000.

31. Oliveira PJ, Seica R, Coxito PM, Rolo AP, Palmeira CM, Santos MS, and Moreno AJ. Enhanced permeability transition explains the reduced calcium uptake in cardiac mitochondria from streptozotocin-induced diabetic rats. FEBS Lett 554: 511514, 2003.

32. Ostrander DB, Zhang M, Mileykovskaya E, Rho M, and Dowhan W. Lack of mitochondrial anionic phospholipids causes an inhibition of translation of protein components of the electron transport chain. A yeast genetic model system for the study of anionic phospholipid function in mitochondria. J Biol Chem 276: 25262-25272, 2001.

33. Palmer JW, Tandler B, and Hoppel CL. Biochemical properties of subsarcolemmal and interfibrillar mitochondria isolated from rat cardiac muscle. J Biol Chem 252: 8731-8739, 1977.

34. Paradies G, Petrosillo G, Paradies V, and Ruggiero FM. Role of cardiolipin peroxidation and $\mathrm{Ca} 2+$ in mitochondrial dysfunction and disease. Cell Calcium 45: 643650, 2009.

35. Paradies G, Petrosillo G, Pistolese M, Di Venosa N, Serena D, and Ruggiero FM. Lipid peroxidation and alterations to oxidative metabolism in mitochondria isolated from rat heart subjected to ischemia and reperfusion. Free Radic Biol Med 27: 42-50, 1999.

36. Paradies G, Ruggiero FM, Dinoi P, Petrosillo G, and Quagliariello E. Decreased cytochrome oxidase activity and changes in phospholipids in heart mitochondria from hypothyroid rats. Arch Biochem Biophys 307: 91-95, 1993.

37. Paradies G, Ruggiero FM, Petrosillo G, and Quagliariello E. Age-dependent decrease in the cytochrome $\mathrm{c}$ oxidase activity and changes in phospholipids in rat-heart mitochondria. Arch Gerontol Geriatr 16: 263-272, 1993.

38. Paradies G, Ruggiero FM, Petrosillo G, and Quagliariello E. Enhanced cytochrome oxidase activity and modification of lipids in heart mitochondria from hyperthyroid rats. Biochim Biophys Acta 1225: 165-170, 1994. 
39. Pullman ME, Penefsky HS, Datta A, and Racker E. Partial resolution of the enzymes catalyzing oxidative phosphorylation. I. Purification and properties of soluble dinitrophenol-stimulated adenosine triphosphatase. J Biol Chem 235: 3322-3329, 1960.

40. Raha S and Robinson BH. Mitochondria, oxygen free radicals, disease and ageing. Trends Biochem Sci 25: 502-508, 2000.

41. Ran Q, Liang H, Gu M, Qi W, Walter CA, Roberts LJ, 2nd, Herman B, Richardson A, and Van Remmen H. Transgenic mice overexpressing glutathione peroxidase 4 are protected against oxidative stress-induced apoptosis. J Biol Chem 279: 55137-55146, 2004.

42. Robinson $\mathbf{A B}$ and Rudd CJ. Deamidation of glutaminyl and asparaginyl residues in peptides and proteins. Curr Top Cell Regul 8: 247-295, 1974.

43. Rolo AP and Palmeira CM. Diabetes and mitochondrial function: role of hyperglycemia and oxidative stress. Toxicol Appl Pharmacol 212: 167-178, 2006.

44. Rosca MG, Okere IA, Sharma N, Stanley WC, Recchia FA, and Hoppel CL. Altered expression of the adenine nucleotide translocase isoforms and decreased ATP synthase activity in skeletal muscle mitochondria in heart failure. J Mol Cell Cardiol 46: 927-935, 2009.

45. Schlame M, Rua D, and Greenberg ML. The biosynthesis and functional role of cardiolipin. Prog Lipid Res 39: 257-288, 2000.

46. Shen X, Zheng S, Thongboonkerd V, Xu M, Pierce WM, Jr., Klein JB, and Epstein PN. Cardiac mitochondrial damage and biogenesis in a chronic model of type 1 diabetes. Am J Physiol Endocrinol Metab 287: E896-905, 2004.

47. Sparagna GC, Johnson CA, McCune SA, Moore RL, and Murphy RC. Quantitation of cardiolipin molecular species in spontaneously hypertensive heart failure rats using electrospray ionization mass spectrometry. J Lipid Res 46: 1196-1204, 2005.

48. Thomas JP, Maiorino M, Ursini F, and Girotti AW. Protective action of phospholipid hydroperoxide glutathione peroxidase against membrane-damaging lipid peroxidation. J Biol Chem 265: 454-461, 1990.

49. Trounce IA, Kim YL, Jun AS, and Wallace DC. Assessment of mitochondrial oxidative phosphorylation in patient muscle biopsies, lymphoblasts, and transmitochondrial cell lines. Methods in enzymology 264: 484-509, 1996. 
50. Ursini F, Maiorino M, Brigelius-Flohe R, Aumann KD, Roveri A, Schomburg D, and Flohe L. Diversity of glutathione peroxidases. Methods Enzymol 252: 38-53, 1995.

51. Wende AR and Abel ED. Lipotoxicity in the heart. Biochim Biophys Acta 1801: 311-319, 2010.

52. Williamson CL, Dabkowski ER, Baseler WA, Croston TL, Alway SE, and Hollander JM. Enhanced apoptotic propensity in diabetic cardiac mitochondria: influence of subcellular spatial location. Am J Physiol Heart Circ Physiol 298: H633-642, 2010.

53. Zhang M, Mileykovskaya E, and Dowhan W. Gluing the respiratory chain together. Cardiolipin is required for supercomplex formation in the inner mitochondrial membrane. J Biol Chem 277: 43553-43556, 2002.

54. Zhou M, Diwu Z, Panchuk-Voloshina N, and Haugland RP. A stable nonfluorescent derivative of resorufin for the fluorometric determination of trace hydrogen peroxide: applications in detecting the activity of phagocyte NADPH oxidase and other oxidases. Anal Biochem 253: 162-168, 1997. 
Figure 6.1

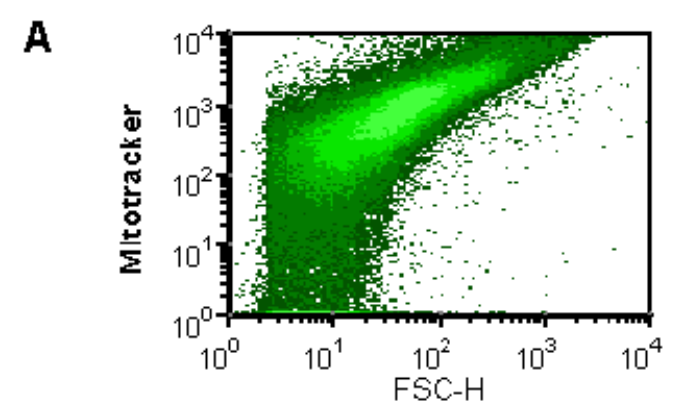

B

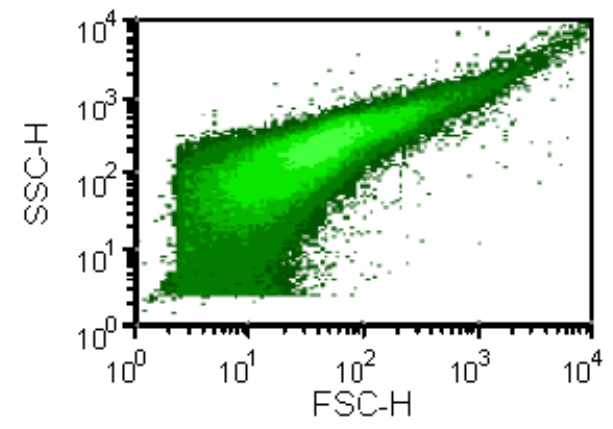

C

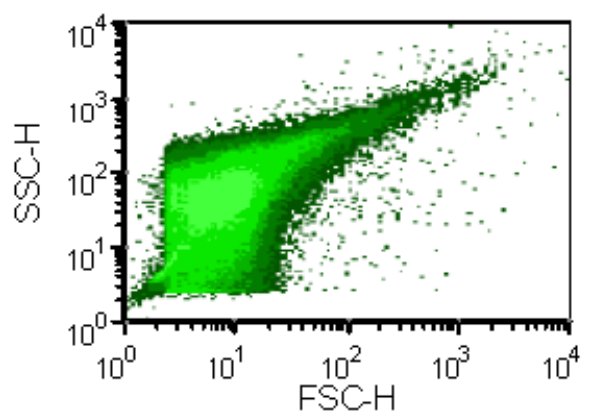


Figure 6.1

Size

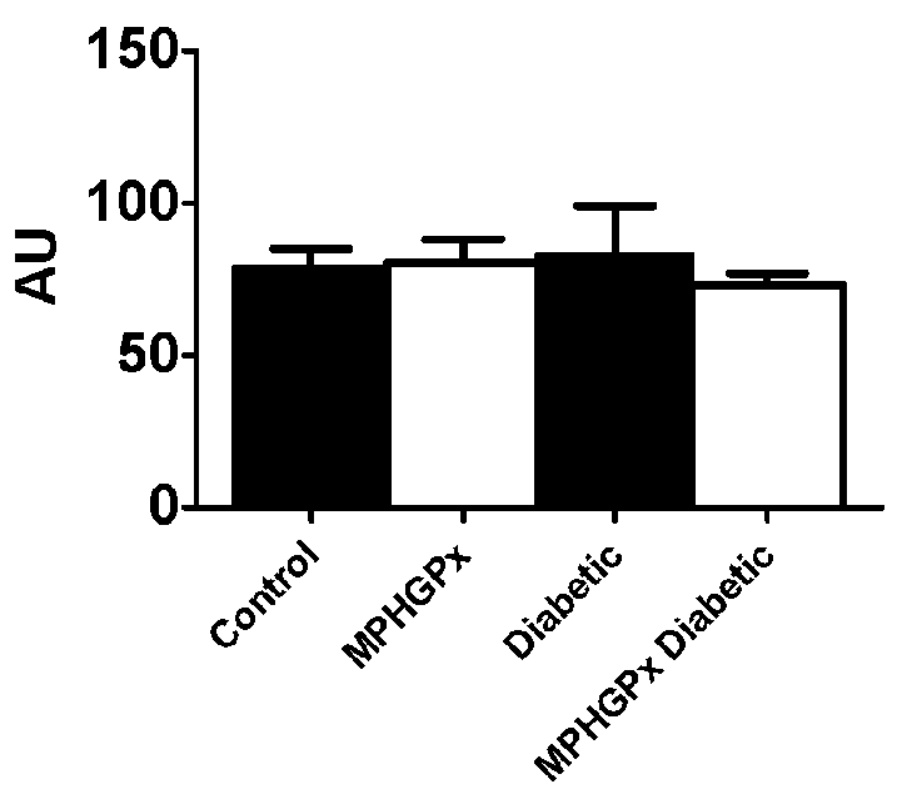

E

Complexity

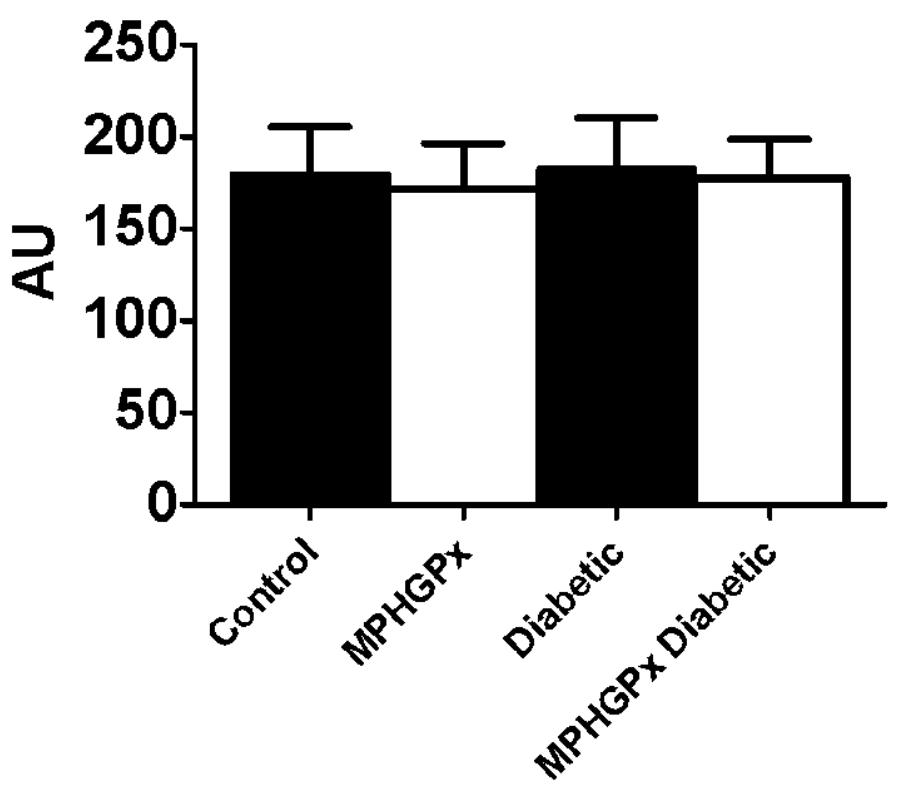


Figure 6.1

F

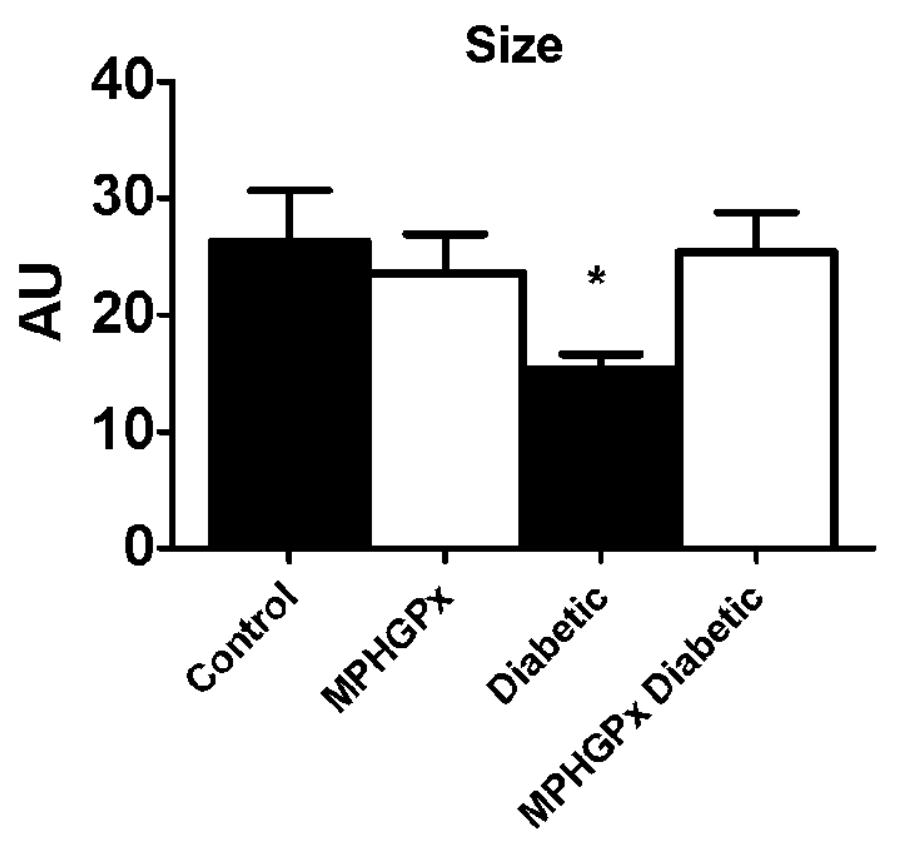

G

Complexity

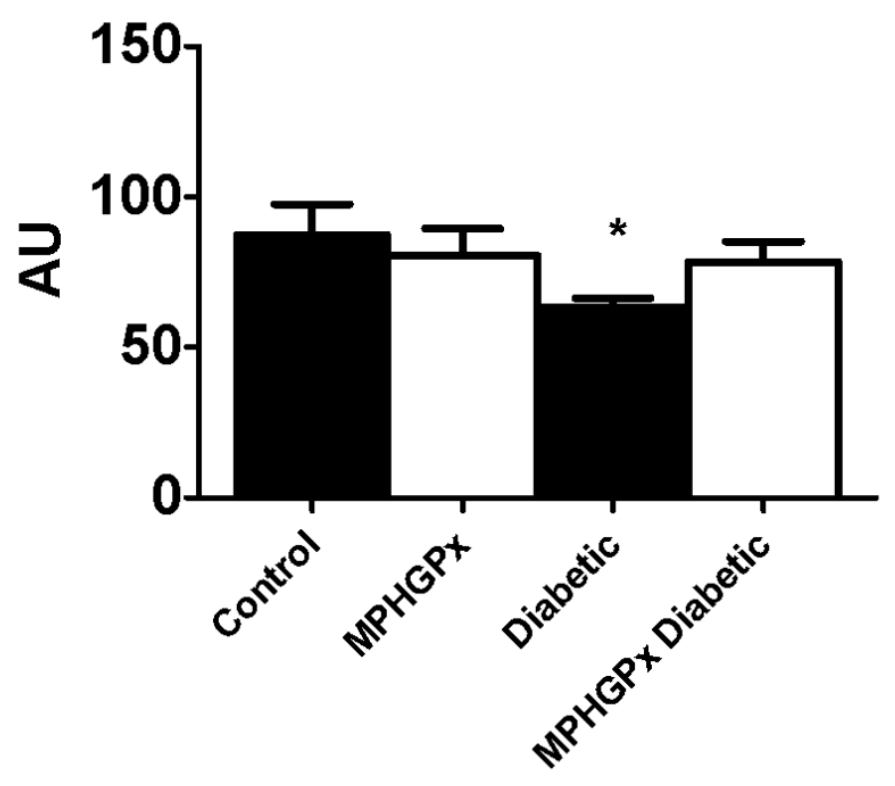


Figure 6.1. Mitochondrial size (FSC) and internal complexity (SSC). Determination of relative size and internal complexity of mitochondrial subpopulations was performed using flow cytometric analyses. Mitochondrial subpopulations from control, diabetic, mPHGPx and mPHGPx diabetic hearts were stained with mitotracker deep Red 633 and gated based on incorporation of the dye. Analysis of FSC and SSC were calculated per 20,000 gated events for samples. Representative dot plots of FSC vs. Mitotracker (A), FSC vs. SSC for SSM (B) and IFM (C). FSC values for SSM (D) and IFM (F) and SSC values for SSM (E) and IFM (G) are expressed as arbitrary units (AU) $\pm \mathrm{SEM} ; \mathrm{N}=5$ for each group. ${ }^{*} P<0.05$ for diabetic vs. all groups. 
Figure 6.2

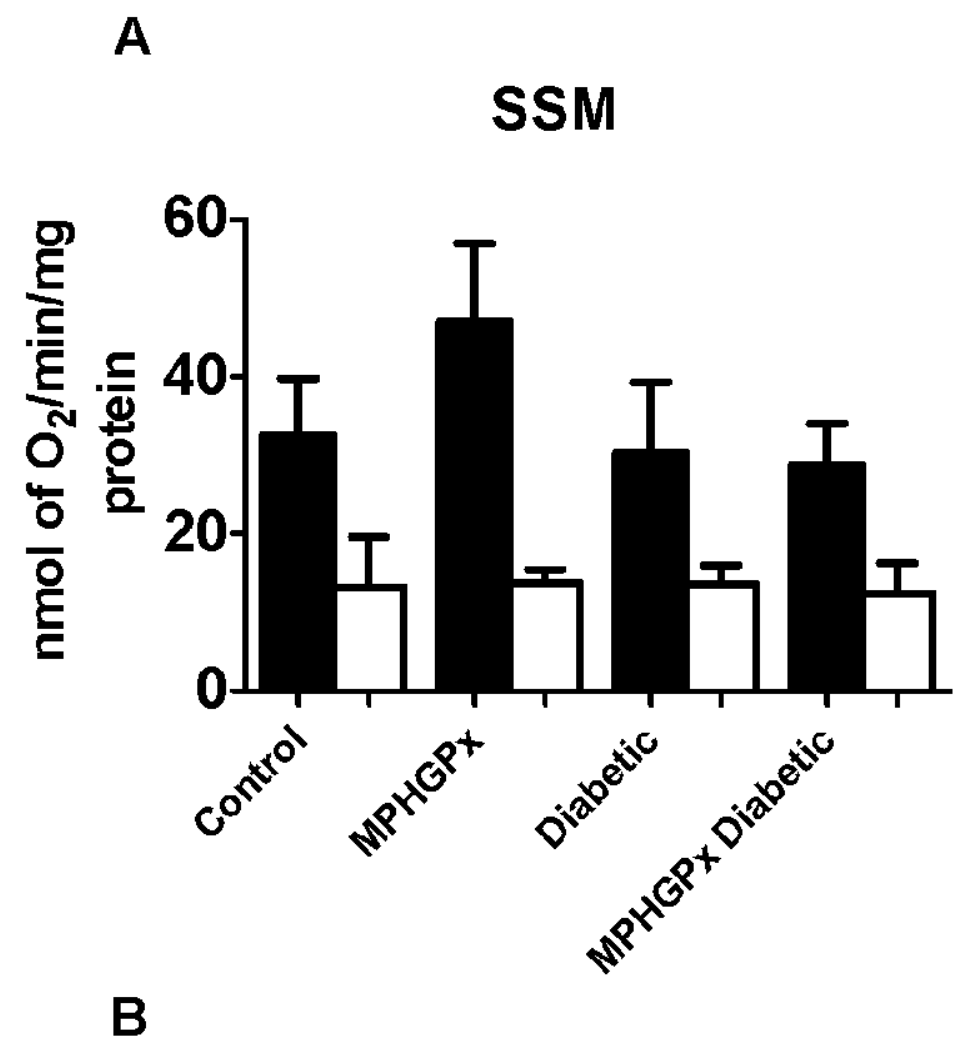

IFM

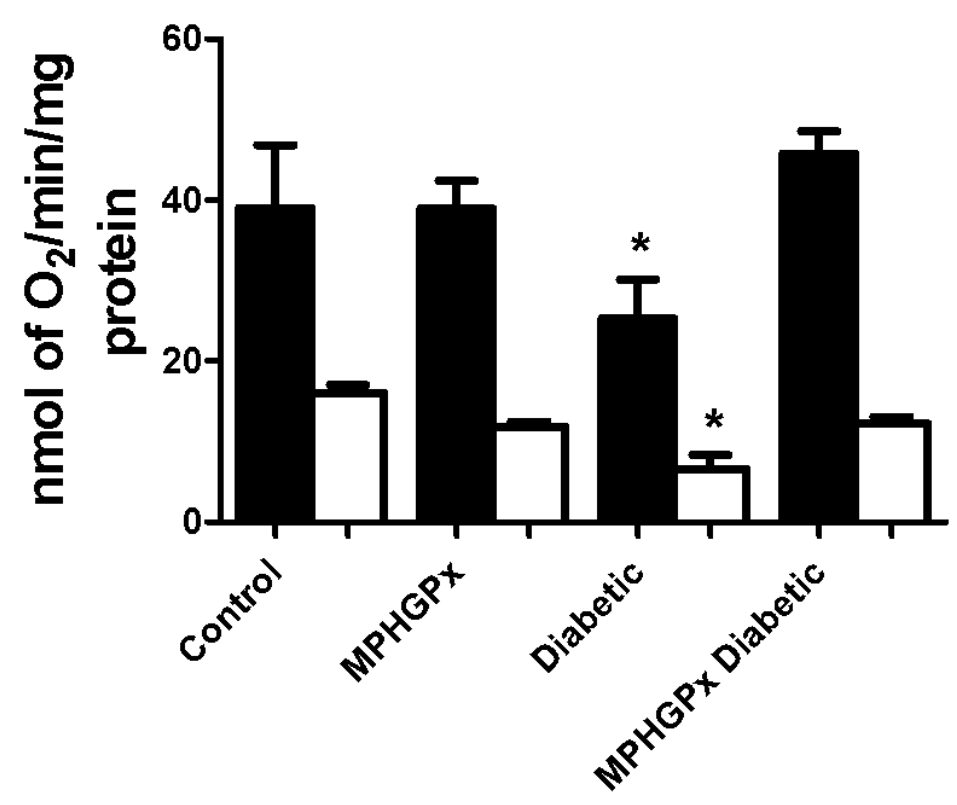


Figure 6.2. Mitochondrial respiration. State 3 (black bars) and state 4 (white bars) respiration rates with glutamate/malate as substrates in SSM (A) and IFM (B) from hearts of control, diabetic, mPHGPx and mPHGPx diabetic animals. State 3 and state 4 respiration rates were determined in the presence of the substrates glutamate/malate and state 3 respiration examined upon addition of ADP. Values are expressed as nanomoles/oxygen $/ \mathrm{min} / \mathrm{mg}$ of protein $\pm \mathrm{SEM}$; $\mathrm{N}=5$ for each group. ${ }^{*} P<0.05$ for diabetic vs. all groups. 
Figure 6.3

A

SSM

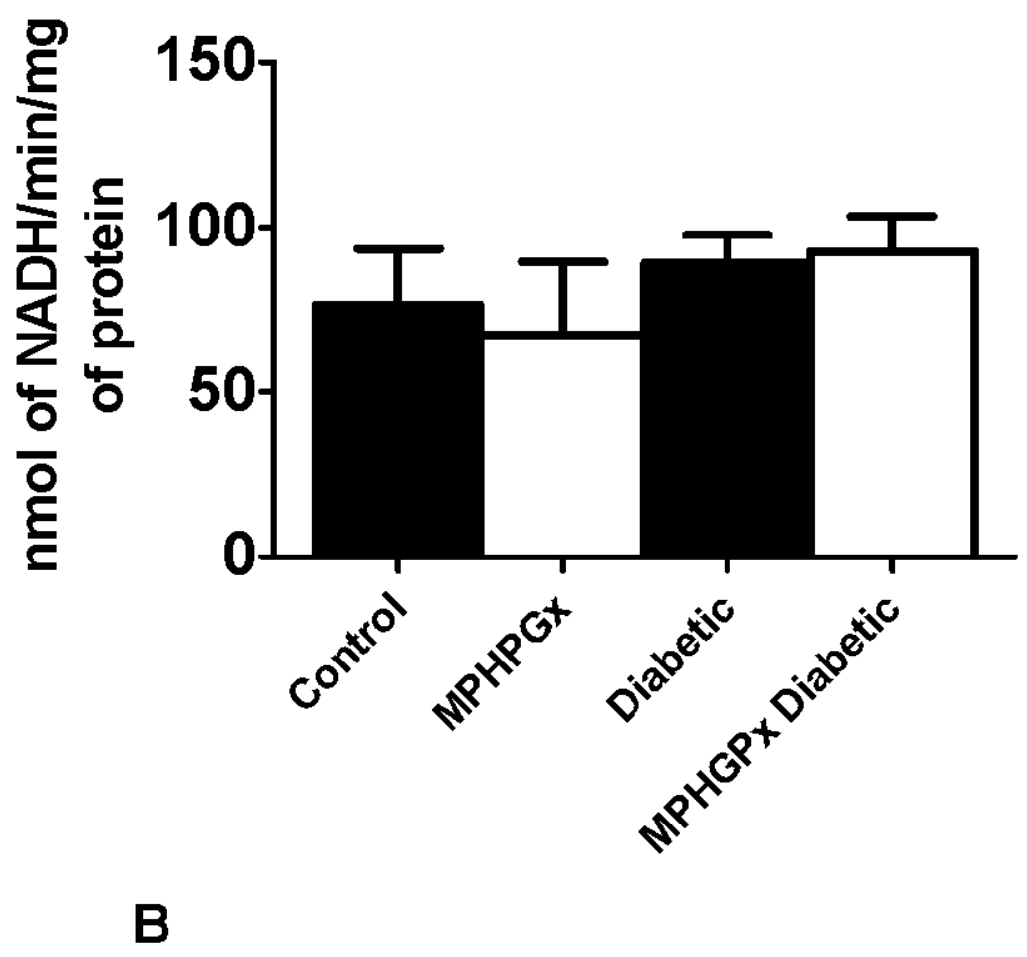

IFM

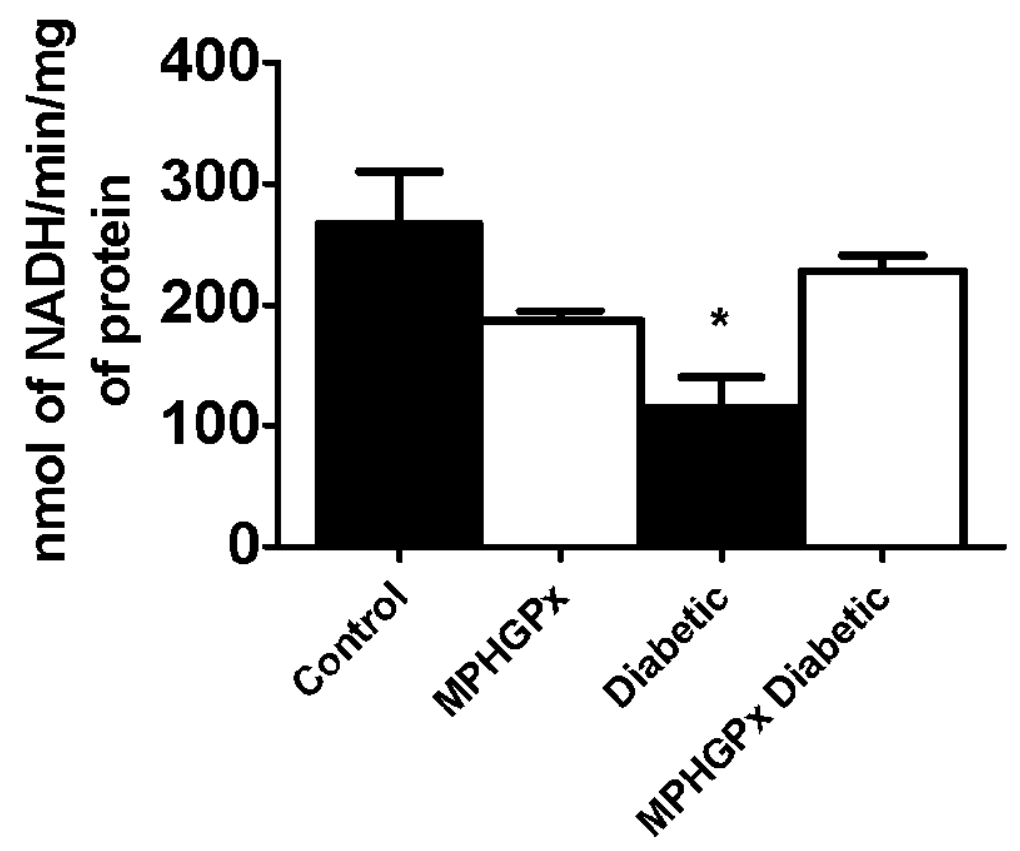


Figure 6.3. ATP synthase activity. ATP synthase activity was assessed in SSM (A) and IFM (B) from hearts of control, mPHGPx, diabetic and mPHGPx diabetic animals using an assay coupled with pyruvate kinase which converts ADP to ATP and produces pyruvate from phosphoenolpyruvate. Final values are expressed as nanomoles $/ \mathrm{NADH} / \mathrm{min} / \mathrm{mg}$ of protein $\pm \mathrm{SEM} ; \mathrm{N}=4$ for each group. ${ }^{*} P<0.05$ for diabetic vs. all groups. 
Figure 6.4

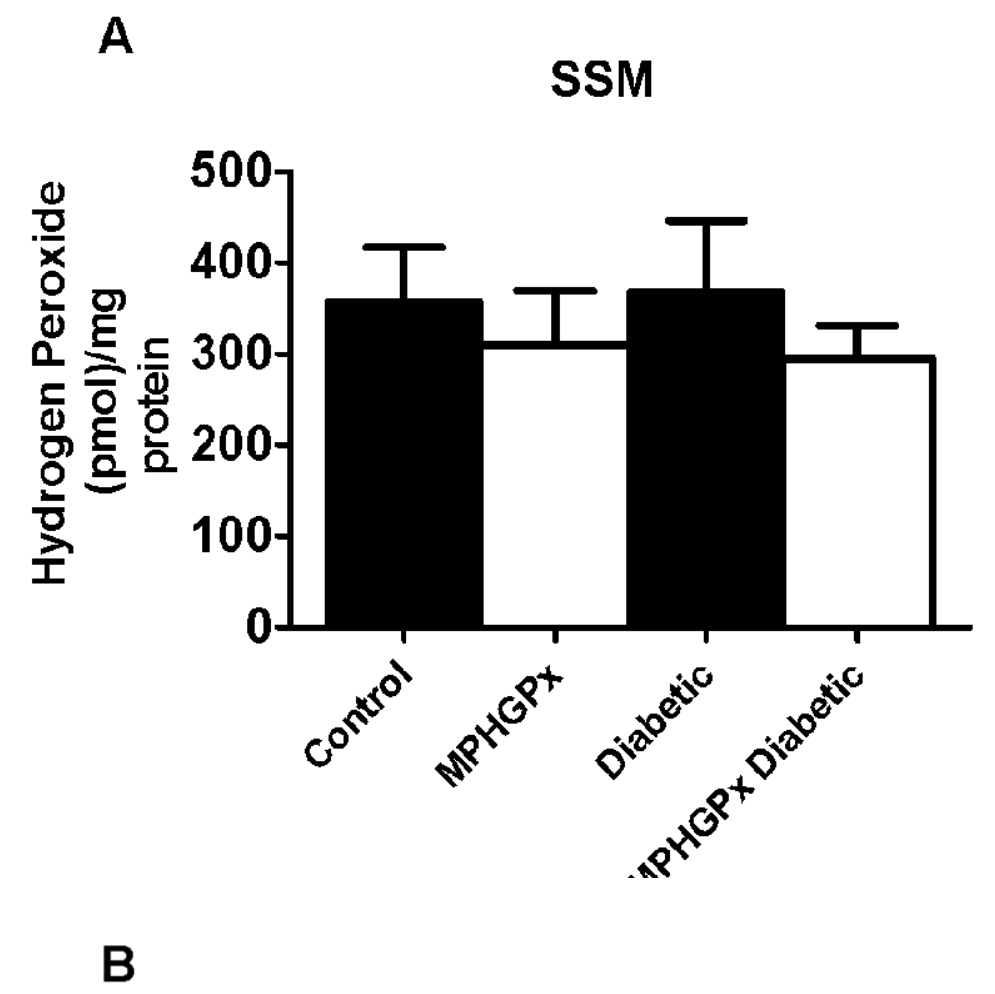

IFM

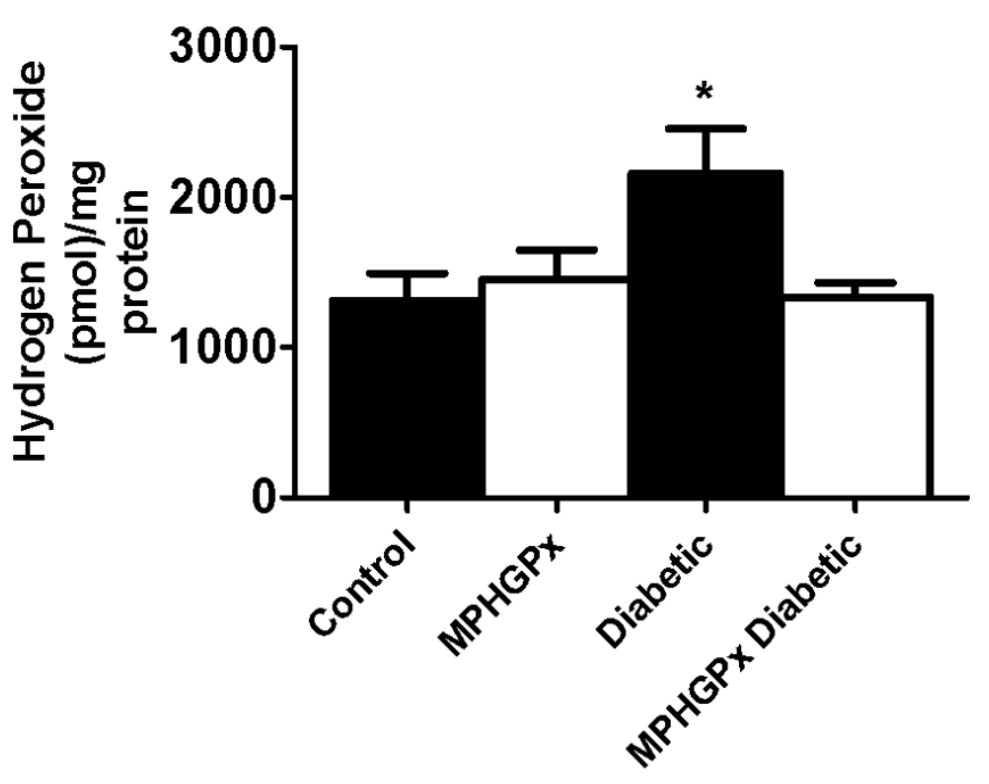


Figure 6.4. Hydrogen peroxide production. $\mathrm{H}_{2} \mathrm{O}_{2}$ production in SSM (A) and IFM (B) was determined using the oxidation of the fluorogenic indicator amplex red in the presence of horseradish peroxidase. $\mathrm{H}_{2} \mathrm{O}_{2}$ production was initiated in mitochondria using glutamate/malate as substrates. Standard curves were obtained by adding known amounts of $\mathrm{H}_{2} \mathrm{O}_{2}$ to the assay medium in the presence of the substrates amplex red and horseradish peroxidase. Final values were calculated as $\mathrm{pmol} / \mathrm{mg}$ of protein. Values are expressed as protein $\pm \mathrm{SEM} ; \mathrm{N}=6$ for each group. ${ }^{*} P<0.05$ for diabetic vs. all groups. 
Figure 6.5
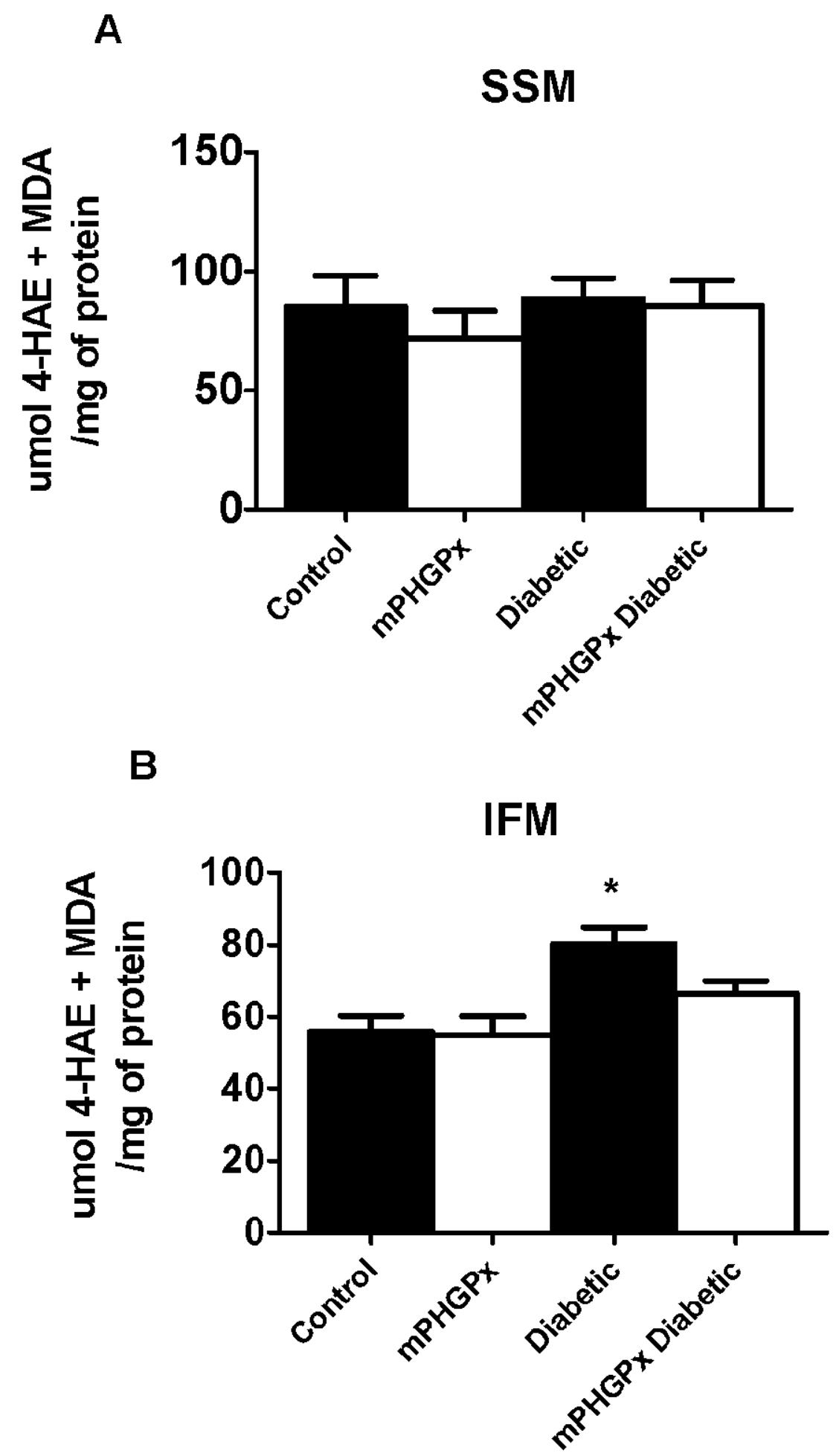
Figure 6.5. Lipid peroxidation by-products. Oxidative damage to lipids was assessed in mitochondrial subpopulations from control, mPHGPx, diabetic, mPHGPx diabetic hearts by measuring lipid peroxidation byproducts malondialdehyde (MDA) and 4-hydroxyalkenals (4-HAE) using a colorimetric assay and compared against a standard curve of known 4-HAE and MDA concentrations. Data are expressed as $\mu \mathrm{mol}$ of MDA + 4-HAE per $\mathrm{mg}$ of mitochondrial protein. Values for lipid peroxidation are expressed as mean $\pm \mathrm{SEM}$; $\mathrm{N}=6$ for each group. ${ }^{*} P<0.05$ for diabetic vs. all groups. 
Figure 6.6

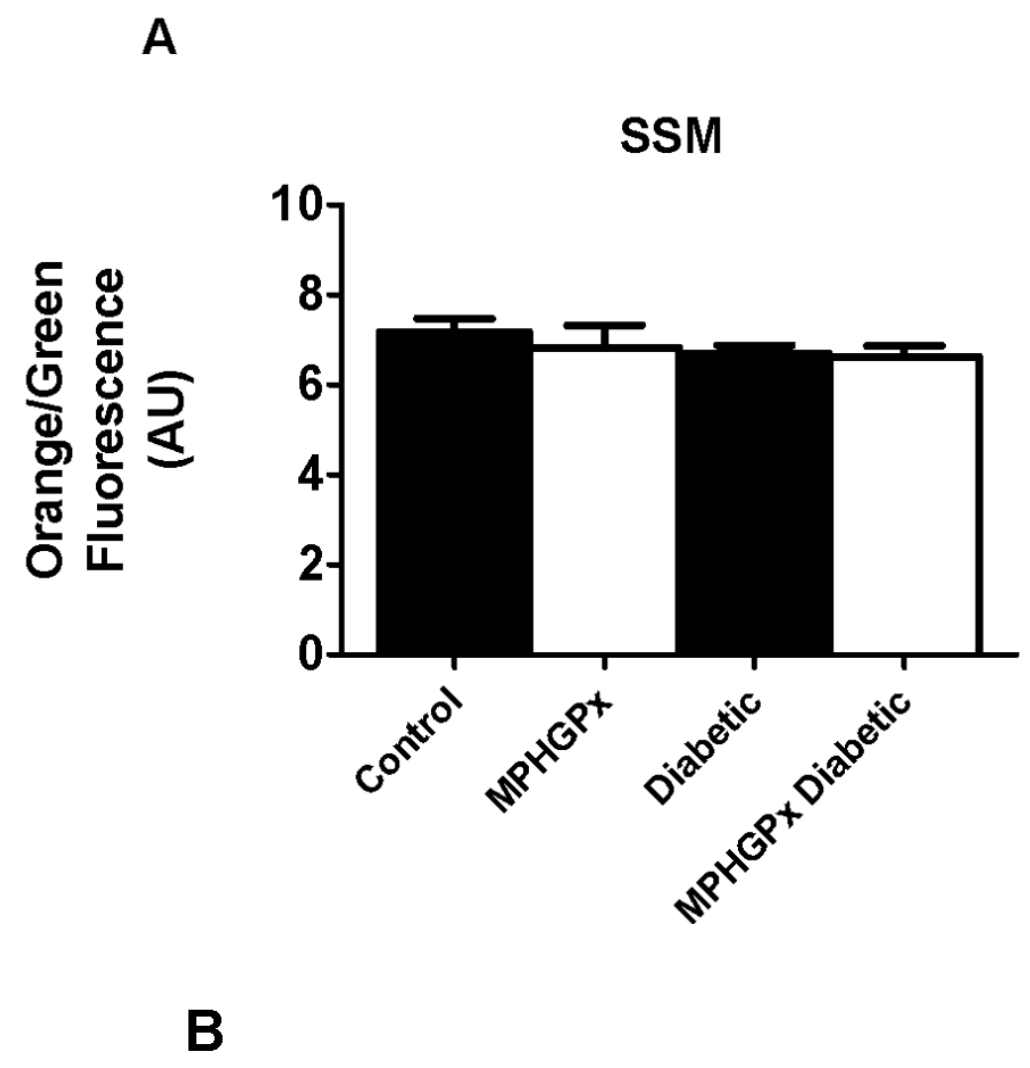

IFM

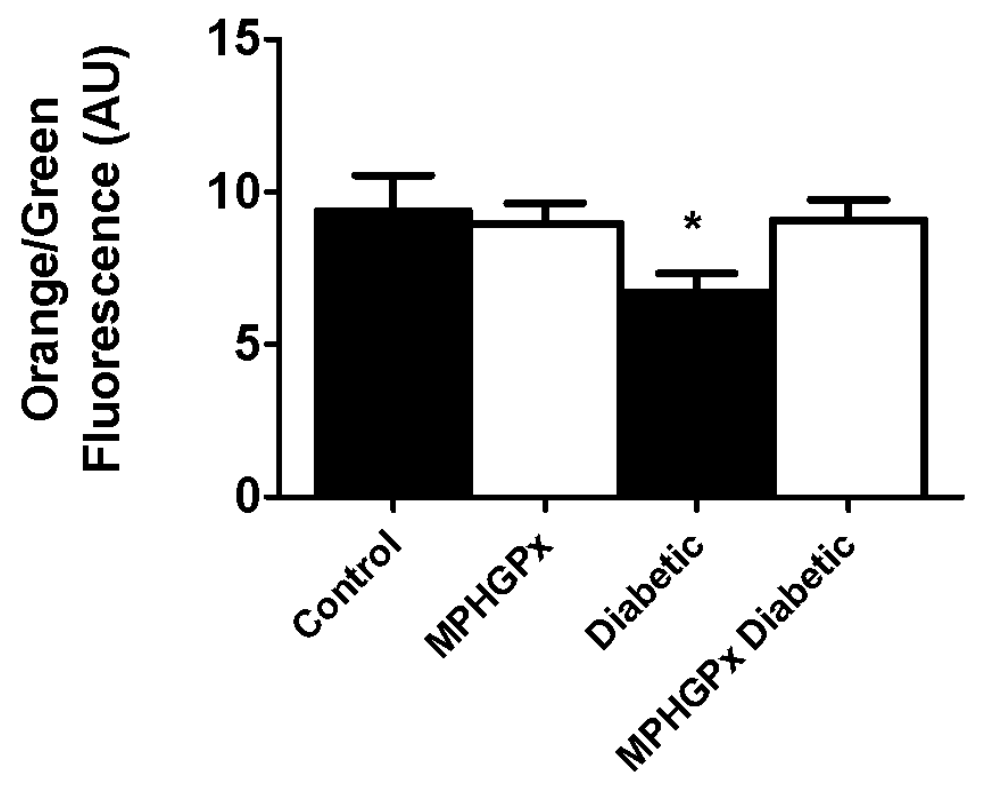


Figure 6.6. Flow cytometric analysis of membrane potential $\left(\Delta \Psi_{\mathrm{m}}\right)$. Membrane potential $\left(\Delta \Psi_{\mathrm{m}}\right)$ was assessed by staining mitochondrial subpopulations with $\mathrm{JC}-1$ dye and assessing the shift from green to orange fluorescence with flow cytometry. $\Delta \Psi_{\mathrm{m}}$ was calculated based on orange/green fluorescence ratios in control, mPHGPx, diabetic, mPHGPx diabetic SSM (A) and IFM (B). Orange/green fluorescence values are expressed as arbitrary units (AU) \pm SEM; $N=6$ for each group. ${ }^{*} P<0.05$ for diabetic vs. all groups. 


\section{Table 6.1}

\begin{tabular}{|l|l|l|l|l|}
\hline Contractile Parameter & Control & Diabetic & MPHGPx & $\begin{array}{l}\text { MPHGPx } \\
\text { Diabetic }\end{array}$ \\
\hline Ejection Fraction (\%) & $89 \pm 1.0$ & $68 \pm 3.9^{*}$ & $83 \pm 3.3$ & $84 \pm .75$ \\
\hline Fractional Shortening (\%) & $54 \pm 1.5$ & $33 \pm 2.8^{*}$ & $46 \pm 3.2$ & $47 \pm .88$ \\
\hline
\end{tabular}


Table 6.1. Cardiac contractile function. Cardiac functional measurements were assessed using echocardiography and are expressed as ejection fraction (\%) and fractional shortening (\%). Values are expressed as means \pm SEM; $N=5$ for each group. ${ }^{*} P<0.05$ for diabetic vs. all groups. 


\section{Table 6.2}

\begin{tabular}{|l|l|l|l|}
\hline Sample & Complex I & Complex III & Complex IV \\
\hline SSM Control & $66 \pm 5$ & $335 \pm 51$ & $1215 \pm 39$ \\
\hline SSM MPHGPx & $58 \pm 7$ & $351 \pm 48$ & $1053 \pm 129$ \\
\hline SSM Diabetic & $68 \pm 6$ & $302 \pm 29$ & $1126 \pm 94$ \\
\hline SSM MPHGPx Diabetic & $56 \pm 5$ & $290 \pm 28$ & $1158 \pm 55$ \\
\hline IFM Control & $90 \pm 7$ & $392 \pm 29$ & $2179 \pm 264$ \\
\hline IFM MPHGPx & $96 \pm 16$ & $434 \pm 59$ & $2560 \pm 334$ \\
\hline IFM Diabetic & $70 \pm 12$ & $301 \pm 22$ & $1386 \pm 45$ \\
\hline IFM MPHGPx Diabetic & $102 \pm 10^{*}$ & $496 \pm 30^{*}$ & $1937 \pm 283^{*}$ \\
\hline
\end{tabular}


Table 6.2. Electron transport chain activity. ETC function was examined in mitochondrial subpopulations from control, mPHGPx, diabetic, mPHGPx diabetic hearts. ETC complex I, III, and IV activities were assessed spectrophotometrically by measuring the oxidation of NADH (complex I), reduction of cytochrome $\mathrm{c}$ (complex III) and oxidation of cytochrome $\mathrm{c}$ (complex IV). Enzymatic activities are expressed as activity $/ \mathrm{min} / \mathrm{mg}$ of protein \pm SEM; $N=6$ for each group. ${ }^{*} P<0.05$ for diabetic vs. all groups. 


\section{Table 6.3}

\begin{tabular}{|c|c|c|}
\hline Protein & Peptide sequence & Modification \\
\hline \multicolumn{3}{|l|}{ Outer Mitochondrial Membrane } \\
\hline $\begin{array}{l}\text { Voltage-dependent anion-selective } \\
\text { channel protein } 2\end{array}$ & VNNSSLIGVGYTQTLRPGVK & Deamidated(N)@3 \\
\hline $\begin{array}{l}\text { Voltage-dependent anion-selective } \\
\text { channel protein } 2\end{array}$ & LTFDTTFSPNTGK & Deamidated(N)@10 \\
\hline Mitochondrial fission 1 protein & MEAVLNELVSVEDLK & $\begin{array}{l}\text { Protein Terminal Acetyl@N- } \\
\text { term }\end{array}$ \\
\hline \multicolumn{3}{|l|}{ Inner Mitochondrial Membrane } \\
\hline ATP synthase subunit alpha & ELLGRVVDALGNAIDGK & $\begin{array}{l}\text { Oxidation(R)@5; } \\
\text { Deamidated(N)@12 }\end{array}$ \\
\hline $\begin{array}{l}\text { ATP synthase, } \mathrm{H}+\text { transporting,F1 } \\
\text { complex, alpha subunit }\end{array}$ & VVDALGNAIDGKGPVGSK & $\begin{array}{l}\text { Deamidated(N)@7; } \\
\text { Oxidation }(\mathrm{P}) @ 14 \\
\end{array}$ \\
\hline ATP synthase subunit beta & IMDPNIVGNEHYDVAR & Deamidated(N)@9 \\
\hline ATP synthase subunit alpha & VVDALGNAIDGK & Deamidated(N)@7 \\
\hline ATP synthase subunit gamma & QMKNEVAALTAAGK & $\begin{array}{l}\text { Gln->pyro-Glu@N-term; } \\
\text { Deamidated(N)@4 }\end{array}$ \\
\hline Cytochrome b-c1 complex subunit 2 & RGNNTTSLLSQSVAK & Deamidated $(\mathrm{N}) @ 3$ \\
\hline Cytochrome b-c1 complex subunit 1 & $\begin{array}{l}\text { TATFAQALQSVPETQVSILD } \\
\text { NGLR }\end{array}$ & Deamidated(N)@21 \\
\hline Cytochrome b-c1 complex subunit 1 & $\begin{array}{l}\text { EMQENDASMQNVVFDYLHA } \\
\text { TAFQGTPLAQAVEGPSENV } \\
\text { R }\end{array}$ & Deamidated(Q)@29 \\
\hline $\begin{array}{l}\text { Cytochrome b-c1 complex subunit } \\
\text { Rieske }\end{array}$ & MLSVAAR & $\begin{array}{l}\text { Protein Terminal Acetyl@N- } \\
\text { term }\end{array}$ \\
\hline Cytochrome c1, heme protein & $\begin{array}{l}\text { ALAEEVEVQDGPNDDGEMF } \\
\text { MRPGK }\end{array}$ & Deamidated(N)@13 \\
\hline Cytochrome $c$ oxidase subunit $5 \mathrm{~A}$ & $\begin{array}{l}\text { AWELRKGMNTLVGYDLVPE } \\
\text { PK }\end{array}$ & $\begin{array}{l}\text { Dioxidation(M)@8; } \\
\text { Deamidated(N)@9 }\end{array}$ \\
\hline Cytochrome $c$ oxidase subunit $5 \mathrm{~A}$ & LNDFASAVR & $\begin{array}{l}\text { Deamidated(N)@2; } \\
\text { Dehydrated(D)@3 }\end{array}$ \\
\hline Cytochrome $c$ oxidase subunit $5 \mathrm{~A}$ & RLNDFASAVR & Deamidated(N)@3 \\
\hline Cytochrome $c$ oxidase subunit $5 \mathrm{~A}$ & YEDSNNLGTSHLLR & Deamidated(N)@6 \\
\hline Cytochrome $c$ oxidase subunit $6 \mathrm{~A} 2$ & GDHGGAGANTWR & Deamidated(N)@9 \\
\hline $\begin{array}{l}\text { NADH-ubiquinone oxidoreductase } 75 \\
\text { kDa subunit }\end{array}$ & GWNILTNSEK & Deamidated(N)@3 \\
\hline $\begin{array}{l}\text { NADH-ubiquinone oxidoreductase } 75 \\
\text { kDa subunit }\end{array}$ & SATYVNTEGR & Deamidated(N)@6 \\
\hline
\end{tabular}




\begin{tabular}{|c|c|c|}
\hline $\begin{array}{l}\text { NADH-ubiquinone oxidoreductase } 75 \\
\text { kDa subunit }\end{array}$ & LTEPMVRNEK & Deamidated(N)@8 \\
\hline $\begin{array}{l}\text { NADH dehydrogenase [ubiquinone] } 1 \\
\text { alpha subcomplex subunit } 7\end{array}$ & NWASGQDLQAK & Deamidated(N)@1 \\
\hline $\begin{array}{l}\text { NADH dehydrogenase [ubiquinone] } \\
\text { flavoprotein } 2\end{array}$ & QNGWLPISAMNK & Deamidated(N)@2 \\
\hline $\begin{array}{l}\text { NADH dehydrogenase [ubiquinone] } \\
\text { iron-sulfur protein } 2\end{array}$ & IDEVEEMLTNNR & Deamidated(N)@10 \\
\hline $\begin{array}{l}\text { NADH dehydrogenase [ubiquinone] } \\
\text { iron-sulfur protein } 2\end{array}$ & $\begin{array}{l}\text { LYTEGYQVPPGATYTAIEAP } \\
\mathrm{K}\end{array}$ & Deamidated(Q)@7 \\
\hline $\begin{array}{l}\text { NADH dehydrogenase [ubiquinone] } 1 \\
\text { alpha subcomplex subunit } 9\end{array}$ & FIHVSHLNASMK & Deamidated(N)@8 \\
\hline $\begin{array}{l}\text { NADH dehydrogenase [ubiquinone] } \\
\text { iron-sulfur protein } 4\end{array}$ & PKPKSKSYGANFSWNKR & Deamidated(N)@15 \\
\hline $\begin{array}{l}\text { NADH dehydrogenase [ubiquinone] } \\
\text { iron-sulfur protein } 4\end{array}$ & NGWSYDVEEK & Deamidated(N)@1 \\
\hline Carnitine O-palmitoyltransferase 2 & $\begin{array}{l}\text { LEDTMKRYLSAQKPLLNDSQ } \\
\text { FR }\end{array}$ & Oxidation(M)@5 \\
\hline Creatine kinase S-type, mitochondrial & $\begin{array}{l}\text { ENLRLQKRGTGGVDTAAVA } \\
\text { DVYDISNIDR }\end{array}$ & $\begin{array}{l}\text { Deamidated(R)@8; } \\
\text { Oxidation(D)@14 }\end{array}$ \\
\hline Chaperone activity of bc1 complex-like & AAMLGDAIMVAK & $\begin{array}{l}\text { Protein Terminal Acetyl@N- } \\
\text { term }\end{array}$ \\
\hline \multicolumn{3}{|l|}{ Matrix-Associated Proteins } \\
\hline Aconitase Hydratase & KGEKNTIVTSYNR & Deamidated(N)@5 \\
\hline Aconitase Hydratase & LEAPDADELPR & Deamidated(N)@1 \\
\hline Aconitase Hydratase & LNRPLTLSEK & Deamidated(N)@1 \\
\hline $\begin{array}{l}\text { 2-oxoglutarate dehydrogenase E1 } \\
\text { component }\end{array}$ & HHVLHDQNVDKR & Deamidated(N)@8 \\
\hline $\begin{array}{l}\text { 2-oxoglutarate dehydrogenase E1 } \\
\text { component }\end{array}$ & NGHNEMDEPMFTQPLMYK & $\begin{array}{l}\text { Deamidated(N)@4; } \\
\text { Deamidated(Q)@13 }\end{array}$ \\
\hline $\begin{array}{l}\text { 2-oxoglutarate dehydrogenase E1 } \\
\text { component }\end{array}$ & $\begin{array}{l}\text { SWDIFFRNTNAGAPPGTAYQ } \\
\text { SPLSLSR }\end{array}$ & $\begin{array}{l}\text { Trioxidation(W)@2; } \\
\text { Deamidated(N)@8 }\end{array}$ \\
\hline $\begin{array}{l}\text { 2-oxoglutarate dehydrogenase E1 } \\
\text { component }\end{array}$ & VIPENGPAAQDPHK & Deamidated(N)@5 \\
\hline $\begin{array}{l}\text { 2-oxoglutarate dehydrogenase E1 } \\
\text { component }\end{array}$ & VIPENGPAAQDPHKVK & Deamidated(N)@5 \\
\hline $\begin{array}{l}\text { 2-oxoglutarate dehydrogenase E1 } \\
\text { component }\end{array}$ & NMEEEVAITR & Deamidated(N)@1 \\
\hline Malate dehydrogenase & $\begin{array}{l}\text { TEVVKAKAGAGSATLSMAYA } \\
\text { GAR }\end{array}$ & Dioxidation(K)@5 \\
\hline
\end{tabular}




\begin{tabular}{|c|c|c|}
\hline Malate dehydrogenase & NLGIGK & Deamidated(N)@1 \\
\hline $60 \mathrm{kDa}$ heat shock protein & $\begin{array}{l}\text { DKYKNIGAKLVQDVANNTNE } \\
\text { EAGDGTTTATVLAR }\end{array}$ & $\begin{array}{l}\text { Didehydroretinylidene(K)@9; } \\
\text { Deamidated(N)@19 }\end{array}$ \\
\hline $60 \mathrm{kDa}$ heat shock protein & TNEEAGDGTTTATVLAR & Deamidated(N)@2 \\
\hline Trifunctional enzyme subunit alpha & INSPNSKVNTLNK & Deamidated(N)@5 \\
\hline Trifunctional enzyme subunit alpha & $\begin{array}{l}\text { MGLVDQLVEPLGPGIKSPEE } \\
\mathrm{R}\end{array}$ & Deamidated(Q)@6 \\
\hline Trifunctional enzyme subunit alpha & GQQQVFKGLNDKVK & Deamidated(Q)@4 \\
\hline $78 \mathrm{kDa}$ glucose-regulated protein & LGGKLSSEDKETMEK & Acetyl@N-term \\
\hline $78 \mathrm{kDa}$ glucose-regulated protein & NQLTSNPENTVFDAK & Deamidated(N)@1 \\
\hline Acetyl-CoA acetyltransferase & KMNISRQEQDTYALSSYTR & Deamidated(Q)@7 \\
\hline Acetyl-CoA acetyltransferase & $\begin{array}{l}\text { AGIPKEEVKEVYMGNVIQGG } \\
\text { EGQAPTR }\end{array}$ & Deamidated(N)@15 \\
\hline $\begin{array}{l}\text { Electron transfer } \\
\text { flavoprotein subunit } \\
\text { beta }\end{array}$ & $\begin{array}{l}\text { VKPDKSGVVTDGVKHSMNP } \\
\mathrm{F}\end{array}$ & Deamidated(N)@18 \\
\hline $\begin{array}{l}\text { Very long-chain specific acyl-CoA } \\
\text { dehydrogenase }\end{array}$ & EATQAVLDKPETLSSDASTR & Deamidated(Q)@4 \\
\hline $\begin{array}{l}\text { Very long-chain specific acyl-CoA } \\
\text { dehydrogenase }\end{array}$ & LCDSWCIEAATR & $\begin{array}{l}\text { Carbamidomethyl(C)@2; } \\
\text { Oxidation(W)@5; } \\
\text { Carbamidomethyl(C)@6 }\end{array}$ \\
\hline $\begin{array}{l}\text { Long-chain specific acyl-CoA } \\
\text { dehydrogenase }\end{array}$ & KFFQEEVIPHHTEWEK & Deamidated(Q)@4 \\
\hline $\begin{array}{l}\text { Long-chain specific acyl-CoA } \\
\text { dehydrogenase }\end{array}$ & $\begin{array}{l}\text { FIPQMTAGKCIGAIAMTEPGA } \\
\text { GSDLQGVR }\end{array}$ & $\begin{array}{l}\text { Oxidation(P)@3; } \\
\text { Deamidated(Q)@4; } \\
\text { Dioxidation(M)@5; } \\
\text { Carbamidomethyl(C)@10 } \\
\end{array}$ \\
\hline $\begin{array}{l}\text { Medium-chain specific acyl-CoA } \\
\text { dehydrogenase }\end{array}$ & MWITNGGK & Deamidated(N)@5 \\
\hline Grp75 & QAVTNPNNTFYATKR & Deamidated(N)@8 \\
\hline 3-ketoacyl-CoA thiolase & $\begin{array}{l}\text { DGTVTAGNASGVSDGAGAVI } \\
\text { IASEDAVKK }\end{array}$ & Deamidated(N)@8 \\
\hline Enoyl-CoA hydratase & GKNSSVGLIQLNRPK & Deamidated(N)@3 \\
\hline $\begin{array}{l}\text { Electron transfer flavoprotein subunit } \\
\text { alpha }\end{array}$ & $\begin{array}{l}\text { GQLRRAASLLRFQSTLVIAE } \\
\text { HANDSLAPITLNTITAAR }\end{array}$ & Deamidated(N)@23 \\
\hline 3,2-trans-enoyl-CoA isomerase & EADIQNFTSFISK & Deamidated(N)@6 \\
\hline
\end{tabular}




\begin{tabular}{|l|l|l|}
\hline $\begin{array}{l}\text { Methylmalonate-semialdehyde } \\
\text { dehydrogenase [acylating] }\end{array}$ & $\begin{array}{l}\text { IVNDNPYGNGTAIFTTNGATA } \\
\text { R }\end{array}$ & $\begin{array}{l}\text { Deamidated(N)@9; } \\
\text { Deamidated(N)@17 }\end{array}$ \\
\hline Peroxiredoxin-5 & FSMVIDNGIVK & $\begin{array}{l}\text { Dethiomethyl(M)@3; } \\
\text { Deamidated(N)@7 }\end{array}$ \\
\hline Fatty acid-binding protein, heart & ADAFVGTWK & $\begin{array}{l}\text { Protein Terminal Acetyl@N- } \\
\text { term }\end{array}$ \\
\hline $\begin{array}{l}\text { Coiled-coil-helix-coiled-coil-helix } \\
\text { domain-containing protein 3 }\end{array}$ & RVTFEADENENITVVK & Deamidated(N)@9 \\
\hline $\begin{array}{l}\text { Delta-1-pyrroline-5-carboxylate } \\
\text { dehydrogenase }\end{array}$ & VANEPILAFSQGSPER & Deamidated(N)@3 \\
\hline $28 S$ ribosomal protein S22 & LMTQAQLEEATR & Deamidated(Q)@4 \\
\hline
\end{tabular}


Table 6.3. Post-translational modifications. Multidimensional protein identification technology (MudPIT) was used to identify post-translational modifications of proteins in IFM control, diabetic and mPHGPx diabetic hearts. Peptides presented represent peptides that contain PTMs in the diabetic IFM but are non-existent with overexpression of mPHGPx in the diabetic heart. 
Table 6.4

\begin{tabular}{|c|c|c|c|c|c|c|c|c|c|}
\hline Cardiolipin & $\begin{array}{l}\text { Analyte Mass } \\
\text { Ranges (amu) }\end{array}$ & $\begin{array}{c}\text { SSM } \\
\text { MPHGPX }\end{array}$ & $\begin{array}{c}\text { SSM } \\
\text { MPHGPX } \\
\text { STZ }\end{array}$ & \begin{tabular}{|c|} 
SSM \\
CONTROL \\
STZ
\end{tabular} & $\begin{array}{c}\text { SSM } \\
\text { CONTROL }\end{array}$ & $\begin{array}{c}\text { IFM } \\
\text { MPHGPX }\end{array}$ & $\begin{array}{c}\text { IFM } \\
\text { MPHGPX } \\
\text { STZ }\end{array}$ & $\begin{array}{c}\text { IFM } \\
\text { CONTROL }\end{array}$ & $\begin{array}{c}\text { IFM } \\
\text { CONTROL } \\
\text { STZ }\end{array}$ \\
\hline C72:9 CL & $1446.0 / 279.2 \mathrm{amu}$ & 1.3 & 1 & 1 & 1.1 & 1.8 & 2.0 & 1.9 & 1.2 \\
\hline C61:1ISTD & $1309.0 / 241.2 \mathrm{amu}$ & 0 & 0 & 0 & 0 & 0.0 & 0.0 & 0.0 & 0.0 \\
\hline C70:7 CL & $1422.0 / 279.2 \mathrm{amu}$ & 0.2 & 0.1 & 0.1 & 0.5 & 1.3 & 0.3 & 2.4 & 0.2 \\
\hline C72:7 CL & $1450.0 / 279.2 \mathrm{amu}$ & 12 & 10.5 & 10.3 & 12.5 & 20.8 & 19.7 & 31.1 & 12.4 \\
\hline C72:8 CL & $1448.0 / 279.2 \mathrm{amu}$ & 46.5 & 44 & 45.2 & 45.4 & 71 & 92.2 & 75 & 57 \\
\hline $\mathrm{C} 80: 12 \mathrm{CL}$ & $1544.0 / 279.2 \mathrm{amu}$ & 0.6 & 0.1 & 0 & 0.2 & 0.5 & 0.2 & 0.8 & 0.1 \\
\hline C74:9 CL & $1474.0 / 279.2 \mathrm{amu}$ & 3 & 0.4 & 0.8 & 3.4 & 6.5 & 7.8 & 7.7 & 3.8 \\
\hline C70:6 CL & $1424.0 / 279.2 \mathrm{amu}$ & 0.3 & 0.2 & 0.3 & 0.4 & 0.8 & 0.5 & 1.4 & 0.4 \\
\hline C76:11 CL & $1498.0 / 279.2 \mathrm{amu}$ & 4.1 & 1.5 & 1 & 1.7 & 3.4 & 2.7 & 6.0 & 1.6 \\
\hline C74:10 CL & $1472.0 / 279.2 \mathrm{amu}$ & 4 & 3.1 & 2.6 & 4.1 & 5.0 & 7.7 & 6.7 & 4.6 \\
\hline C74:8 CL & $1476.0 / 279.2 \mathrm{amu}$ & 6 & 3.1 & 4.3 & 4.3 & 6.0 & 8.8 & 7.2 & 4.9 \\
\hline $\mathrm{C} 72: 6 \mathrm{CL}$ & $1452.0 / 279.2 \mathrm{amu}$ & 4.6 & 3.5 & 3 & 3.3 & 5.4 & 5.0 & 7.7 & 3.3 \\
\hline C78:14 CL & $1520.0 / 279.2 \mathrm{amu}$ & 0.9 & 0.2 & 0.1 & 0.4 & 0.9 & 0.7 & 1.3 & 0.3 \\
\hline C78:13 CL & $1522.0 / 303.2 \mathrm{amu}$ & 0 & 0 & 0 & 0 & 0.0 & 0.0 & 0.0 & 0.0 \\
\hline C80:14 CL & $1548.0 / 303.2 \mathrm{amu}$ & 0 & 0 & 0 & 0 & 0.0 & 0.0 & 0.0 & 0.0 \\
\hline C80:4ISTD & $1569.2 / 337.4 \mathrm{amu}$ & 0 & 0 & 0 & 0 & 0.0 & 0.0 & 0.0 & 0.0 \\
\hline C76:10 & $1500.0 / 281.2 \mathrm{amu}$ & 0.3 & 0.1 & 0 & 0.2 & 0.3 & 0.2 & 0.5 & 0.1 \\
\hline C72:5 & $1454.0 / 281.2 \mathrm{amu}$ & 0.6 & 0.2 & 0.3 & 0.4 & 0.7 & 0.6 & 1.1 & 0.4 \\
\hline C76:12 CL & $1496.0 / 279.2 \mathrm{amu}$ & 4.5 & 3.1 & 1.1 & 5.4 & 10.3 & 7.5 & 10.8 & 14.3 \\
\hline TOTAL & & 88.9 & 71.1 & 70.1 & 83.3 & 134.7 & 155.9 & 161.6 & 104.6 \\
\hline
\end{tabular}


Table 6.4. Cardiolipin content. Multiple cardiolipin species were assessed in mitochondrial subpopulations from control, mPHGPx, diabetic, mPHGPx diabetic hearts. The most predominant cardiolipin species is represented at an analyte mass range of 1448, while the oxidized cardiolipin species is at an analyte mass range of 1496 . Samples represented were a pooled $n=5$ for each group. 


\section{Chapter 7:}

\section{General Discussion}




\section{GENERAL DISCUSSION}

The overall objective of this dissertation was to determine the influence of spatial location on mitochondrial dysfunction in the diabetic heart. We further wanted to gain insight into whether a mitochondrially-targeted antioxidant, mPHGPx, could provide protection in the diabetic heart. Our long term goal was to mechanistically determine how mitochondrial dysfunction is associated with the pathogenesis of diabetes mellitus and to examine whether specifically targeted therapeutics are protective. The Central Hypothesis of this dissertation was that the IFM would be more susceptible to diabetesassociated dysfunction and ultimately contribute to overall mitochondrial and contractile dysfunction present with both type 1 and type 2 diabetes mellitus. Further, we hypothesized that overexpression of a mitochondrial specific antioxidant capable of preserving the IMM may serve as a therapeutic. Our rationale for the proposed research was to elucidate differential mechanisms and key components of mitochondrial dysfunction with diabetes mellitus that ultimately will aid in developing more spatially targeted therapeutics.

Mitochondrial dysfunction has been suggested to play a major role in the pathogenesis of type 1 and type 2 DM [1-4]. However, this dysfunction is complicated by the fact that two distinct subpopulations of mitochondria exist in the heart. Several authors have observed and suggested that these two spatially-distinct subpopulations respond differently with various physiological and pathological stimuli including aging, $\mathrm{I} / \mathrm{R}$, exercise, caloric restriction, high fat feeding and heart failure [2, 3, 5-13]. King et al. [11] first reported significantly diminished state 3 and state 4 respiration in both SSM and IFM from STZ-induced rat heart which is somewhat in contrast to our initial study (Chapter 2), suggesting the IFM had greater respiratory dysfunction than the SSM. Differences between the two studies may be due to differences in the animal model used, rat vs. mouse, and substrates used for respiration measurements. Further, in our analyses, we examined a multitude of biochemical characteristics that were not present in the King et al. [11] study such as ROS generation, oxidative damage, morphology and cardiolipin content. To date, no other examination of mitochondrial subpopulations has been made in either a type 1 or type 2 diabetic model. One of the most intriguing 
aspects of this dissertation was the fact that mitochondria located in different areas of the cell were impacted differentially with type 1 and type 2 DM. Chapters 2 and 3 were focused on examining mitochondrial subpopulation specific dysfunction in STZ-induced type 1 diabetic mice. We hypothesized that there would be an increase in oxidative stress and damage resulting in diminished mitochondrial membrane integrity, accompanied by overall mitochondrial dysfunction in both diabetic mitochondrial subpopulations. More specifically, we believed that IFM would be more susceptible with STZ-induced DM primarily due to their higher respiratory rates and subsequent oxidative environment. Due to the IFM's primary role in ATP production for contractile function, we believed IFM would contribute to diabetic cardiomyopathy-associated dysfunction to a greater extent than the SSM. In agreement with our hypothesis, mitochondrial dysfunction was greatest in the IFM from the type 1 diabetic heart. We observed this through decreases in mitochondrial size and internal complexity (Chapter 2) and diminished overall ETC function. We also observed an increase in ROS generation and enhanced oxidative damage to proteins and lipids within the IFM. Cardiolipin, a phospholipid within the IMM essential for proper mitochondrial function, was also decreased in abundance specifically in the IFM. It is important to note that there were some differences observed in the SSM, however, changes were much greater in the IFM. Taken together, our results suggested that with type $1 \mathrm{DM}$, both subpopulations seemed to be dysfunctional with the effect being greatest in the IFM. Because the IFM population provides ATP for contractile processes [14], the compromise in ETC function and enhanced oxidative stress may be particularly detrimental to cardiac contractile function, contributing to the contractile deficits associated with diabetic cardiomyopathy. Our initial findings in the type 1 diabetic model were complicated by the fact that we saw the opposite phenomenon with type 2 DM (Chapter 4), in that the SSM were more dysfunctional. In contrast to our findings in the type 1 diabetic heart, the SSM had decreases in size/complexity, decreased overall respiratory capacity and enhanced oxidative damage to lipids and proteins with no changes in the IFM. Reasons for the mitochondrial subpopulation specific differences between type 1 and type 2 DM are not clearly understood. These findings are probably 
due to differences in the phenotypes elicited by the two conditions, resulting from the unique pathological etiologies specific for each model.

Though both type 1 and type 2 DM manifest through decreased cardiac glucose utilization and a switch to excessive FA utilization, key differences exist between type 1 and type 2 diabetic phenotypes. In regards to cardiac contractile function, both diastolic and systolic dysfunction are observed in STZ-induced type 1 diabetic animals between 4-6 weeks post hyperglycemia onset $[4,15,16]$. It is believed this is mainly due to prolonged hypoinsulinemia and chronic hyperglycemia. On the other hand, with various models of type $2 \mathrm{DM}$, there are conflicting reports, with some studies documenting both impaired systolic and diastolic function, whereas other studies argue against a change in systolic function $[17,18]$. It is possible with Type 2 diabetic models, this discrepancy could be due to the severity or duration of hyperinsulinemia, and transient hyperglycemia.

It is important to point out that in multiple models of both type $1 \mathrm{DM}[3,19,20]$ and type 2 DM [1] increased ROS formation appears to be an underlying contributor to mitochondrial dysfunction. However, the mechanism in which enhanced oxidative stress manifests may be different between type 1 and type 2 DM. Altered substrate utilization (mainly increased FAO) and enhanced oxidative stress precedes onset of hyperglycemia in the $\mathrm{db} / \mathrm{db}$ mouse model $[1,21]$. It is suggested that pooling of neutral fatty acids within the mitochondria contribute to enhanced ROS formation [22]. On the other hand, hyperglycemia is present and believed to contribute to enhanced ROS production in the STZ mouse model. Persistently high levels of glucose in the STZ model can enhance ROS formation which inturn, increases polyol pathway flux, advanced-glycation end product formation, protein kinase $C$ and hexosamine pathway flux, all of which contribute to cardiac dysfunction [23]. Further, in the $\mathrm{db} / \mathrm{db}$ model of type $2 \mathrm{DM}$, lipid profiles are much more dramatically increased than in the STZ-induced type 1 diabetic mice. Db/db mice have higher circulating fatty acids (Chapter 4 ) and triglycerides than the STZ mice. Nielson et al. observed lipid accumulation specifically surrounding the SSM in type 2 diabetic skeletal muscle suggesting that the SSM may be subject to a more enhanced lipid environment in the type 2 diabetic state based upon its proximity [24]. Higher free fatty acids and lipid deposition associated with the 
subsarcolemmal region during type $2 \mathrm{DM}$ may put the SSM at greater risk due to their location at the cell periphery as compared to IFM [25]. It is plausible that an increased lipid environment in and surrounding the SSM may enhance the oxidative environment within the SSM contributing to their dysfunction. Such a phenomenon would manifest during type $2 \mathrm{DM}$ to a greater extent with an enhanced lipid environment as compared to type $1 \mathrm{DM}$. Further, it is important to point out that diminished function in either subpopulation may have a negative impact on cardiac contraction regardless of subpopulation. Though it has been speculated that each subpopulation provides ATP for specific cellular functions, inability to provide ATP for any of these functions may translate into contractile abnormalities.

Proteomic analyses (Chapter 3, Chapter 4) were then utilized to gain further insight into other potential contributors to the differences observed between the two subpopulations of mitochondria with DM. Proteomic analyses have played an integral role in furthering our understanding of mitochondrial dysfunction in the diabetic heart. Several proteomic studies, in a variety of diabetic models, have been employed to assess proteomic changes associated with the diabetic heart [26-31]. However, we are the first to examine subpopulation-specific proteomic differences in type 1 diabetic and type 2 diabetic models.

In the initial proteomic analyses of the mitochondrial subpopulations from the type 1 diabetic hearts, we utilized both 2D-DIGE and iTRAQ approaches. Utilizing ITRAQ and 2D-DIGE methodologies allowed a number of advantages over the use of a single proteomic methodology including the ability to confirm experimental results and accuracy by essentially comparing results from each method. By using MudPIT analysis combined with ITRAQ labeling, the limitations associated with 2D-DIGE, such as solubility and separation, were bypassed. Further, the ITRAQ approach enabled comparison of total protein levels, which can be more difficult using 2D-DIGE separation, where PTMs such as phosphorylation can alter pls and hence gel migration [28]. Utilizing 2D-DIGE allowed us to examine proteins that may be in less abundance and as a result, less likely to show up with ITRAQ analyses. Because each method has its own unique set of advantages, the combined use of both techniques enhanced our level of confidence in the reported findings. In later studies, iTRAQ was used in 
assessing mitochondrial subpopulations from the type 2 diabetic hearts mainly due to lack of 2D-DIGE resources. Nevertheless, results from both studies were very interesting. The majority of proteins identified as changing with both 2D-DIGE and ITRAQ approaches in the type 1 diabetic IFM were FAO and ETC proteins. In total, we identified 9 ETC proteins from ITRAQ analyses and 16 ETC proteins from 2D-DIGE analyses as being decreased in the diabetic IFM. Jullig et al. reported decreases in ETC constituents that are involved in oxidative phosphorylation in total mitochondria [28], which is somewhat different from our findings examining mitochondrial subpopulations, emphasizing the importance of taking into account subcellular location when examining mitochondria. Further, translocases located within the IMM that are essential for energy production through synthesis (mitochondrial phosphate carrier) and transport of ATP (ANT1) were also significantly decreased only in the diabetic IFM. Our proteomic data were consistent with previous reports of decreased respiratory capacity in the type 1 diabetic heart as well as functional data indicating down-regulation of ETC activities, specifically in the IFM $[3,11,29]$. Down-regulation of multiple components of the oxidative phosphorylation machinery and FAO proteins suggests that mitochondria in the type 1 diabetic heart, specifically IFM, may be less efficient in producing ATP required for contraction and calcium homeostasis. In the type 2 hearts, we see a similar phenomenon but in the SSM. Using the iTRAQ ${ }^{\mathrm{TM}}$ approach, ETC complex proteins were primarily decreased in $\mathrm{db} / \mathrm{db}$ SSM, while ETC and TCA cycle proteins were increased in the IFM. The results indicated that protein loss in the SSM may be contributing to the decrease in oxidative capacity observed in the SSM. Further, the majority of the proteins lost in the db/db SSM reside in the IMM, including ETC proteins, which may have contributed to the decrease in oxidative capacity observed in the SSM. Taken together, these results suggest that the formation and/or organization of ETC constituents contribute to the respiratory dysfunction observed in both IFM (type 1 DM) and SSM (type $2 \mathrm{DM}$ ). In the type 2 diabetic hearts, we further observed an increase in FAO proteins in both SSM and IFM of db/db hearts (Chapter 4), which is probably due, in part, to the enhanced lipid environment present with type 2 DM [32]. However, though the effect occurred in both subpopulations, the result was more pronounced in the SSM, which saw a greater number of FAO proteins increased in abundance, 
suggesting a more enhanced fatty acid environment presented to this subpopulation. As stated earlier, due to the fact that the SSM are located at the periphery of the cell, they may be impacted to a greater extent by an enhanced lipid environment, as compared to IFM, which reside in the interior of the cell. However, it is important to point out that though we observed upregulation of FAO proteins in the SSM, the ability to utilize fatty acid substrates (palmitoylcarnitine) was diminished in the SSM suggesting that the mitochondrial dysfunction displayed by $\mathrm{db} / \mathrm{db}$ SSM may be due to limitations to the ETC as opposed to FAO. In the type 1 diabetic IFM, FAO proteins were decreased suggesting another potential difference between the two etiologies. In general, a majority of proteins were decreased in abundance in the IFM from the type 1 diabetic heart. This suggests that proteomic dysregulation with type 1 and type 2 DM may be through very different mechanisms. However, of interest is the fact the Grp75, a key contributor of nuclear-encoded protein import, was decreased in both the type 1 diabetic IFM and the type 2 diabetic SSM suggesting a mechanism of proteomic alterations with both pathologies. This finding is intriguing and warrants further investigation.

Potential mechanisms of proteomic dysregulation may include alterations in gene expression, enhanced PTMs, or upregulation of regulators such as miRNAs [33, 34]. With both type 1 and type 2 DM, PTMs identified included oxidations, deamidations, deacetylations and methylations, with oxidations and deamidations being the most prevalent. Interestingly, the diabetic IFM displayed the greatest number of oxidativelymodified proteins in the type 1 diabetic hearts while the SSM contained the most oxidations in the type 2 diabetic hearts. However, the type 1 diabetic IFM had a greater number of oxidations identified than the type 2 diabetic SSM. This suggests that the IFM may have a more enhanced oxidative environment in the type 1 diabetic heart than the SSM in the type 2 diabetic heart. Another PTM that is associated with oxidative stress that was prevalent in both the type 1 diabetic IFM and type 2 diabetic SSM was deamidation. It has been suggested that deamidation of asparaginyl and glutaminyl residues within proteins causes structural and biological alterations to peptide and protein structure [35]. Though the contribution of deamidation and oxidation to protein dysfunction is somewhat unclear, these PTMs may alter protein structure/function making the protein more susceptible for degradation by the proteasome system $[35,36]$. 
It is plausible that these modifications may have contributed to altered functionality and subsequent proteasomal degradation, all of which may have contributed to the morphological and oxidative phosphorylation deficiencies present in both the type 1 and type 2 diabetic hearts.

Of greatest interest from both proteomic analyses (protein abundance and PTMs) is the observation that proteins of the IMM, an important locus containing the ETC, ATP synthesis machinery, and protein trafficking constituents, were specifically impacted in the SSM as a result of type $2 \mathrm{DM}$ and in the IFM as a result of type $1 \mathrm{DM}$. Interestingly, only $21 \%$ of total mitochondrial proteins are located within the IMM, while $69 \%$ are located in the matrix as shown below [37]

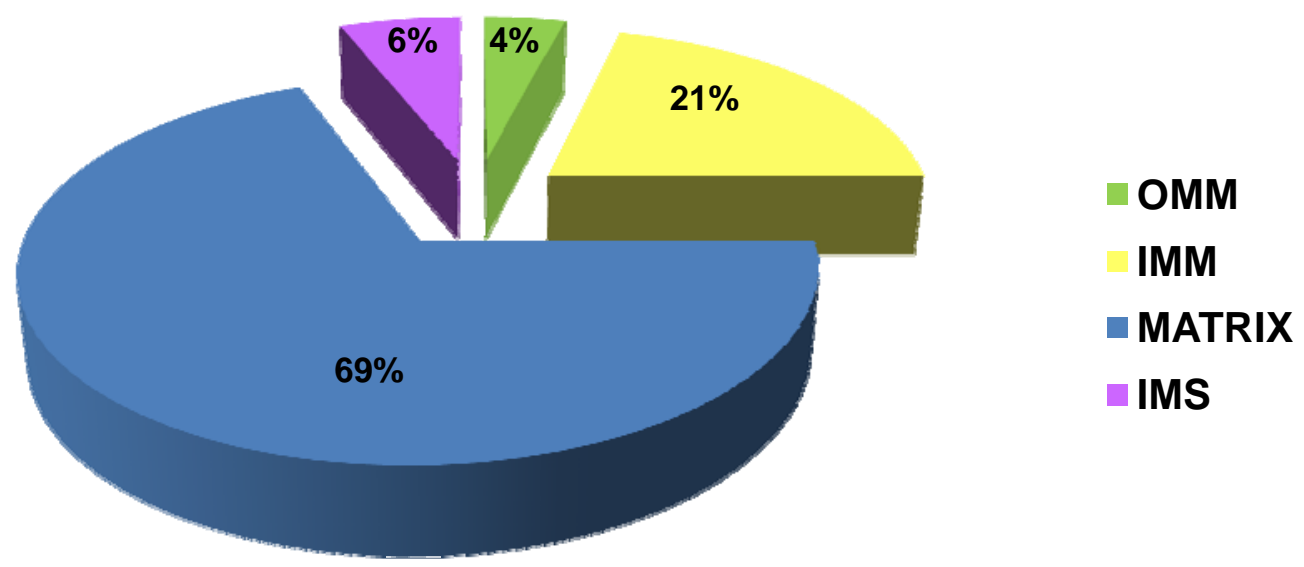

Figure 7.1. The percentage of proteins abundant in each mitochondrial subcompartment including OMM (4\%), IMS (6\%), MATRIX (69\%) and IMM (21\%).

However, in our proteomic analysis of mitochondrial protein abundance, we observed that approximately $50 \%$ of proteins changing in both the type 1 diabetic IFM (Figure 7.2A) and type 2 diabetic SSM (Figure 7.2B) were IMM proteins, with only approximately $45 \%$ of matrix proteins being affected. 


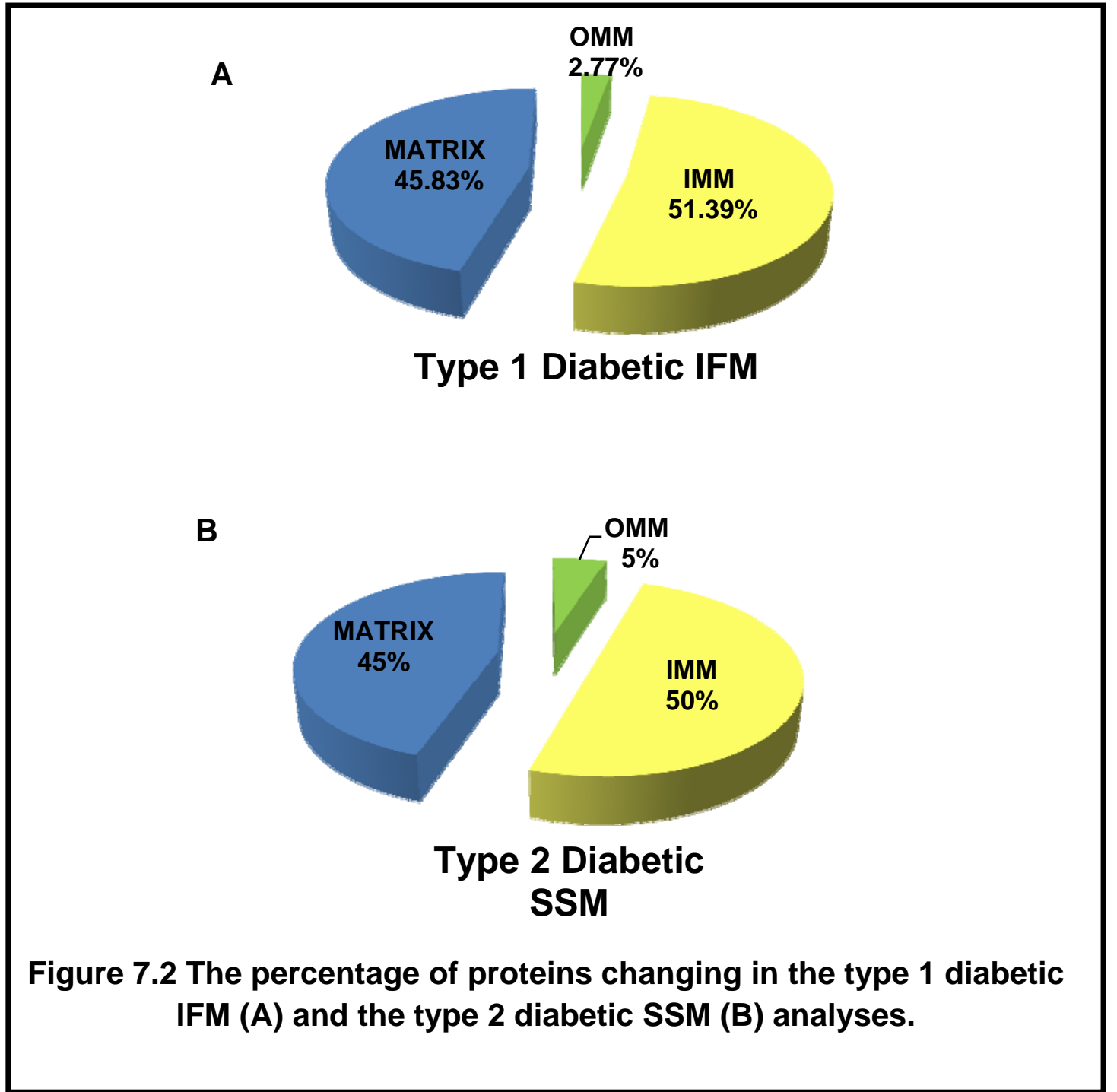

This emphasizes that fact the IMM is specifically being affected in both type 1 and type 2 DM regardless of the fact the less proteins reside in the IMM as compared to the matrix.

Further, functional aspects of the IMM, such as $\Delta \Psi \mathrm{m}$, respiration, ETC activities, and ATP synthesis were concomitantly diminished in the IFM with type 1 DM and in the SSM with type $2 \mathrm{DM}$ strengthening the argument that the IMM is at risk in the diabetic heart. Taken together, the combination of findings provided by our studies offer evidence that not all cardiac mitochondria are impacted equally by diabetic insult (either type 1 or type 2), but rather mitochondrial dysfunction is specific to a particular subcellular locale. Our analyses of mitochondrial subpopulation proteomic 
compositional changes resulting from type 1 and type 2 diabetic insult reveals submitochondrial loci at risk, and identifies specific proteomic targets that may be of interest for therapeutic and diagnostic interventions.

Because our initial findings indicated that the IMM seems to be at risk with both type 1 and type 2 DM associated dysfunction, we then explored whether overexpression of a key mitochondrially-targeted antioxidant enzyme, mPHGPx, could provide protection in the type 1 diabetic heart. MPHGPx is unique in that it is the only antioxidant enzyme capable of reducing peroxidized acyl groups in phospholipids, fatty acid hydroperoxides and cholesterol peroxides in mitochondrial membranes [38]. Since $\mathrm{mPHGPx}$ is a lipophilic enzyme, it can interact directly with lipid hydroperoxides within the IMM, and it has been suggested to be the primary antioxidant defense against biomembrane oxidation within the mitochondrion [39]. Our data suggests that there is an enhanced oxidative environment within the mitochondria that is associated with increased oxidative damage to proteins and lipids within the type 1 diabetic heart. The data further suggests that the IMM and its protein constituents, including ETC proteins and cardiolipin, seem to be particularly at risk. Cardiolipin is a diphosphatidylglycerol enriched in the inner membrane, containing oxidatively-sensitive acyl groups that may act as selective targets for ROS [40]. Cardiolipin plays an essential role of stabilizing protein complexes and translocases within the IMM, facilitating proper mitochondrial function. Han et al. demonstrated dramatic loss of abundant cardiolipin molecular species in STZ-treated hearts at the very earliest stages of DM accompanied by a profound remodeling of the remaining $C L$ molecular species [41]. Remodeling of cardiolipin can certainly have a positive or negative impact on function and the susceptibility of being oxidatively modified. Future experiments examining remodeling of cardiolipin species at earlier time points may provide insight into cardiolipin aberrations in the type 1 diabetic heart.

Damage to these proteins and lipids within the IMM contributes to mitochondrial dysfunction by limiting sufficient production of ATP. As a result, we wanted to examine whether mitochondria-specific overexpression of PHGPx could attenuate ROS-induced damage to the mitochondria by preservation of the IMM structure and function which 
inturn, improves cardiac contractile function in the diabetic heart. We hypothesized that overexpression of $\mathrm{mPHGPx}$ will decrease oxidative stress and damage with type $1 \mathrm{DM}$. More specifically, overall mitochondrial structure and function will be protected through preservation of the phospholipid bi-layer including cardiolipin a phospholipid critical to membrane integrity and the stabilization of the ETC. Our findings were in agreement with our hypothesis that overexpression of MPHGPx would preserve overall cardiac and mitochondrial function in the type 1 diabetic heart (Chapter 6). Specifically, cardiac contractile function, mitochondrial morphology and mitochondrial respiratory capacity were all preserved in the IFM with overexpression of $\mathrm{MPHGPx}$ in the diabetic heart. Further, overexpression of mPHGPx in the diabetic heart also increased the most predominant species of cardiolipin specifically in the IFM suggesting the ability of mPHPGx to preserve cardiolipin content. Interestingly, the oxidized form of cardiolipin was increased in the diabetic IFM. Overexpression of MPHGPx decreased the content of oxidized cardiolipin which inturn, increased the ratio of non-oxidized to oxidized cardiolipin in the diabetic IFM. Oxidative damage to cardiolipin would be detrimental and alter IMM dynamics such as membrane fluidity, ion permeability, surface charge, membrane enzyme activity and could even initiate apoptosis [42]. Because cardiolipin is necessary for IMM function and integrity, preservation of cardiolipin may be a potential mechanism in which $\mathrm{mPHGPx}$ provides protection in the diabetic heart.

Taken together, these data suggest that mPHGPx provides protection in the type 1 diabetic heart via preserved mitochondrial dynamics. Figure 7.3 represents the proposed mechanism in which we believe overexpression of mPHGPx preserves 


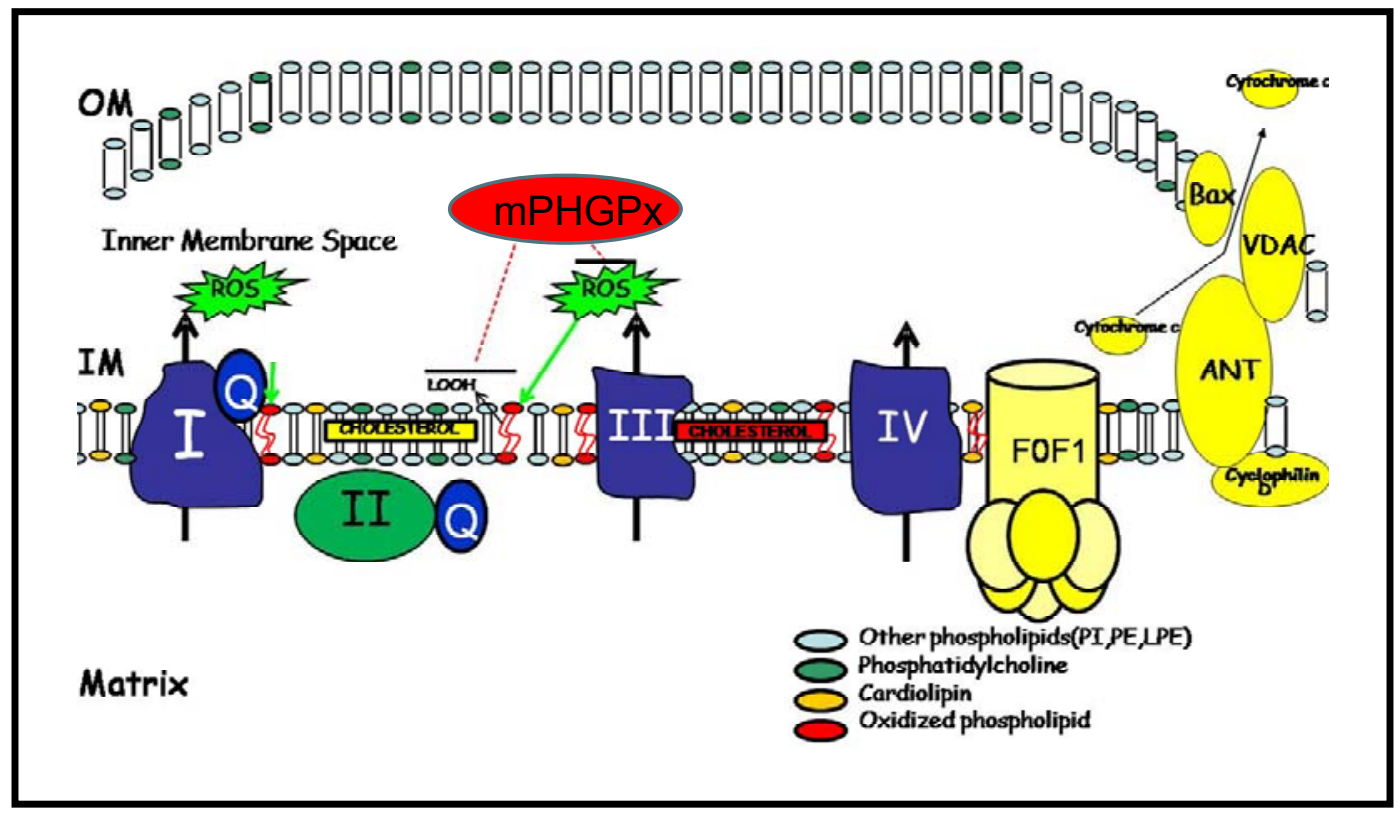

Figure 7.3. MPHGPx Mechanism of Protection

mitochondrial function, which inturn, translates into adequate energy production and improved contractility in the diabetic heart. Overexpression of mPHGPx is capable of inhibiting ROS induced damage to key protein and lipid constituents within the IMM. Further, we believe that preservation of the phospholipid bi-layer, including cardiolipin, stabilizes the ETC proteins, retains adequate ATP production and prevents mitochondrial dysfunction with type $1 \mathrm{DM}$.

In terms of clinical application, MPHGPx mimetics may offer therapeutic advantages over other mitochondrial targeted antioxidants. Several small-scale clinical trials have suggested the efficacy of the natural antioxidants a-tocopherol (vitamin E), ascorbate (vitamin C), Coenzyme $Q$, and $\alpha$-lipoic acid $[43,44]$ with various degenerative diseases. However, the uptake and distribution to tissues of hydrophobic natural antioxidants is often poor [45]. Development of artificial antioxidants, such as mimetics of MnSOD or mPHGPx, would be more potent than natural antioxidants and also have improved bioavailability and stability [46]. An advantage of using mPHGPx over 
MnSOD is the fact that $\mathrm{mPHGPx}$ can directly interact with the main source of ROS generation, the IMM, and prevent accumulation of very reactive lipid hydroperoxides.

In conclusion, our data suggests that subcellular location must be taken into account when examining mitochondrial dysfunction in the diabetic heart. In particular, mitochondria located between the myofibrils are more dysfunctional in a type 1 diabetic setting, while those mitochondria located at the cell periphery have greater dysfunction in a type 2 diabetic setting. Further, our data suggests that the IMM is particularly at risk with DM induced dysfunction and overexpression of a mitochondrially-targeted antioxidant enzyme capable of preserving the IMM provides cardioprotection. Thus, development of antioxidants targeted not only to subcellular but also suborganelle locations may provide protection in the diabetic heart. 


\section{References}

1. Boudina, S., et al., Mitochondrial energetics in the heart in obesity-related diabetes: direct evidence for increased uncoupled respiration and activation of uncoupling proteins. Diabetes, 2007. 56(10): p. 2457-66.

2. Dabkowski, E.R., et al., Mitochondrial Dysfunction in the Type 2 Diabetic Heart is Associated with Alterations in Spatially-Distinct Mitochondrial Proteomes. Am J Physiol Heart Circ Physiol, 2010.

3. Dabkowski, E.R., et al., Diabetic cardiomyopathy-associated dysfunction in spatially distinct mitochondrial subpopulations. Am J Physiol Heart Circ Physiol, 2009. 296(2): p. H359-69.

4. Flarsheim, C.E., I.L. Grupp, and M.A. Matlib, Mitochondrial dysfunction accompanies diastolic dysfunction in diabetic rat heart. Am J Physiol, 1996. 271(1 Pt 2): p. H192-202.

5. Leeuwenburgh, C., et al., Age-related differences in apoptosis with disuse atrophy in soleus muscle. Am J Physiol Regul Integr Comp Physiol, 2005. 288(5): p. R1288-96.

6. Adhihetty, P.J., et al., Differential susceptibility of subsarcolemmal and intermyofibrillar mitochondria to apoptotic stimuli. Am J Physiol Cell Physiol, 2005. 289(4): p. C994-C1001.

7. Chen, Q., et al., Ischemic defects in the electron transport chain increase the production of reactive oxygen species from isolated rat heart mitochondria. Am J Physiol Cell Physiol, 2008. 294(2): p. C460-6.

8. Fannin, S.W., et al., Aging selectively decreases oxidative capacity in rat heart interfibrillar mitochondria. Arch Biochem Biophys, 1999. 372(2): p. 399-407.

9. Judge, S., et al., Age-associated increases in oxidative stress and antioxidant enzyme activities in cardiac interfibrillar mitochondria: implications for the mitochondrial theory of aging. Faseb J, 2005. 19(3): p. 419-21.

10. Kelley, D.E., et al., Dysfunction of mitochondria in human skeletal muscle in type 2 diabetes. Diabetes, 2002. 51(10): p. 2944-50. 
11. King, K.L., et al., Diabetes or peroxisome proliferator-activated receptor alpha agonist increases mitochondrial thioesterase I activity in heart. J Lipid Res, 2007. 48(7): p. 1511-7.

12. Lesnefsky, E.J., et al., Ischemia, rather than reperfusion, inhibits respiration through cytochrome oxidase in the isolated, perfused rabbit heart: role of cardiolipin. Am J Physiol Heart Circ Physiol, 2004. 287(1): p. H258-67.

13. Ritov, V.B., et al., Deficiency of subsarcolemmal mitochondria in obesity and type 2 diabetes. Diabetes, 2005. 54(1): p. 8-14.

14. Palmer, J.W., B. Tandler, and C.L. Hoppel, Biochemical properties of subsarcolemmal and interfibrillar mitochondria isolated from rat cardiac muscle. J Biol Chem, 1977. 252(23): p. 8731-9.

15. Bugger, H. and E.D. Abel, Rodent models of diabetic cardiomyopathy. Dis Model Mech, 2009. 2(9-10): p. 454-66.

16. An, D. and B. Rodrigues, Role of changes in cardiac metabolism in development of diabetic cardiomyopathy. Am J Physiol Heart Circ Physiol, 2006. 291(4): p. H1489-506.

17. Dong, F., et al., Impaired cardiac contractile function in ventricular myocytes from leptin-deficient ob/ob obese mice. J Endocrinol, 2006. 188(1): p. 25-36.

18. Semeniuk, L.M., A.J. Kryski, and D.L. Severson, Echocardiographic assessment of cardiac function in diabetic $d b / d b$ and transgenic $d b / d b-h G L U T 4$ mice. Am J Physiol Heart Circ Physiol, 2002. 283(3): p. H976-82.

19. Kaul, N., et al., Probucol improves antioxidant activity and modulates development of diabetic cardiomyopathy. Nutrition, 1995. 11(5 Suppl): p. 551-4.

20. Shen, X., et al., Cardiomyocyte defects in diabetic models and protection with cardiac-targeted transgenes. Methods Mol Med, 2005. 112: p. 379-88.

21. Boudina, S. and E.D. Abel, Diabetic cardiomyopathy, causes and effects. Rev Endocr Metab Disord, 2010. 11(1): p. 31-9.

22. Wende, A.R. and E.D. Abel, Lipotoxicity in the heart. Biochim Biophys Acta, 2010. 1801(3): p. 311-9. 
23. Rolo, A.P. and C.M. Palmeira, Diabetes and mitochondrial function: role of hyperglycemia and oxidative stress. Toxicol Appl Pharmacol, 2006. 212(2): p. 167-78.

24. Nielsen, J., et al., Increased subsarcolemmal lipids in type 2 diabetes: effect of training on localization of lipids, mitochondria, and glycogen in sedentary human skeletal muscle. Am J Physiol Endocrinol Metab. 298(3): p. E706-13.

25. Nielsen, J., et al., Increased subsarcolemmal lipids in type 2 diabetes: effect of training on localization of lipids, mitochondria, and glycogen in sedentary human skeletal muscle. Am J Physiol Endocrinol Metab, 2010. 298(3): p. E706-13.

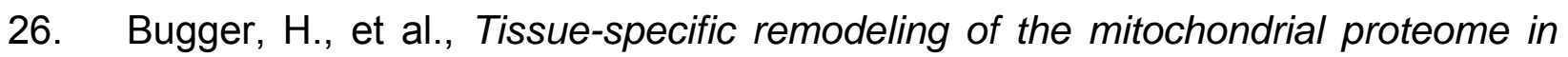
type 1 diabetic akita mice. Diabetes, 2009. 58(9): p. 1986-97.

27. Hamblin, M., et al., Alterations in the diabetic myocardial proteome coupled with increased myocardial oxidative stress underlies diabetic cardiomyopathy. J Mol Cell Cardiol, 2007. 42(4): p. 884-95.

28. Jüllig, M., et al., Characterization of proteomic changes in cardiac mitochondria in streptozotocin-diabetic rats using iTRAQ $\angle$ SUP $><F O N T$ SIZE='$1^{\prime}>T M</ F O N T></ S U P>$ isobaric tags. PROTEOMICS - Clinical Applications, 2007. 1(6): p. 565-576.

29. Shen, X., et al., Cardiac mitochondrial damage and biogenesis in a chronic model of type 1 diabetes. Am J Physiol Endocrinol Metab, 2004. 287(5): p. E896905.

30. Turko, I.V. and F. Murad, Quantitative protein profiling in heart mitochondria from diabetic rats. J Biol Chem, 2003. 278(37): p. 35844-9.

31. Dabkowski, E.R., et al., Mitochondrial Dysfunction in the Type 2 Diabetic Heart is Associated with Alterations in Spatially-Distinct Mitochondrial Proteomes. Am J Physiol Heart Circ Physiol, 2010: p. ajpheart.00267.2010.

32. Abdul-Ghani, M.A., et al., Deleterious action of FA metabolites on ATP synthesis: possible link between lipotoxicity, mitochondrial dysfunction, and insulin resistance. Am J Physiol Endocrinol Metab, 2008. 295(3): p. E678-85. 
33. Nishi, H., et al., MicroRNA-15b modulates cellular ATP levels and degenerates mitochondria via Arl2 in neonatal rat cardiac myocytes. J Biol Chem, 2010. 285(7): p. 4920-30.

34. Kane, L.A. and J.E. Van Eyk, Post-translational modifications of ATP synthase in the heart: biology and function. J Bioenerg Biomembr, 2009. 41(2): p. 145-50.

35. Robinson, A.B. and C.J. Rudd, Deamidation of glutaminyl and asparaginyl residues in peptides and proteins. Curr Top Cell Regul, 1974. 8(0): p. 247-95.

36. Iwai, K., et al., Iron-dependent oxidation, ubiquitination, and degradation of iron regulatory protein 2: implications for degradation of oxidized proteins. Proc Natl Acad Sci U S A, 1998. 95(9): p. 4924-8.

37. Distler, A.M., J. Kerner, and C.L. Hoppel, Proteomics of mitochondrial inner and outer membranes. Proteomics, 2008. 8(19): p. 4066-82.

38. Lu, J. and A. Holmgren, Selenoproteins. J Biol Chem, 2009. 284(2): p. 723-7.

39. Thomas, J.P., et al., Protective action of phospholipid hydroperoxide glutathione peroxidase against membrane-damaging lipid peroxidation. In situ reduction of phospholipid and cholesterol hydroperoxides. J Biol Chem, 1990. 265(1): p. 45461.

40. Houtkooper, R.H. and F.M. Vaz, Cardiolipin, the heart of mitochondrial metabolism. Cell Mol Life Sci, 2008. 65(16): p. 2493-506.

41. Han, X., et al., Alterations in myocardial cardiolipin content and composition occur at the very earliest stages of diabetes: a shotgun lipidomics study. Biochemistry, 2007. 46(21): p. 6417-28.

42. Paradies, G., et al., Role of cardiolipin peroxidation and Ca2+ in mitochondrial dysfunction and disease. Cell Calcium, 2009. 45(6): p. 643-50.

43. Rosen, P., et al., The role of oxidative stress in the onset and progression of diabetes and its complications: a summary of a Congress Series sponsored by UNESCO-MCBN, the American Diabetes Association and the German Diabetes Society. Diabetes Metab Res Rev, 2001. 17(3): p. 189-212.

44. West, I.C., Radicals and oxidative stress in diabetes. Diabet Med, 2000. 17(3): p. $171-80$. 
45. Bentinger, M., et al., Distribution and breakdown of labeled coenzyme Q10 in rat. Free Radic Biol Med, 2003. 34(5): p. 563-75.

46. Green, K., M.D. Brand, and M.P. Murphy, Prevention of mitochondrial oxidative damage as a therapeutic strategy in diabetes. Diabetes, 2004. 53 Suppl 1: p. S110-8. 


\title{
Curriculum Vitae
}

\author{
Erinne R. Dabkowski
}

\section{OFFICE ADDRESS}

Division of Exercise Physiology and

Center for Interdisciplinary Research in

Cardiovascular Sciences

West Virginia University School of Medicine

P.O. Box 9227

Morgantown, WV 26506

Tel: (304) 293-3683

Fax: (304) 293-7105

Email: edabkowski@hsc.wvu.edu

\section{EDUCATION}

2005-Present Doctor of Philosophy, Exercise Physiology, West Virginia University School of Medicine

2001-2005

Bachelors of Science, Biological Sciences, Clemson University, Clemson South Carolina

\section{POSITIONS}

2010

Graduate student representative on biomedical Sciences admissions committee 


\section{FELLOWSHIPS AND AWARDS}

2002-2003 Clemson University current exchange program, Aberdeen Scotland

$2008 \quad$ West Virginia University School of Medicine Biomedical Sciences travel award

2008

Accepted to be on $\mathrm{NIH}$ predoctoral training grant Cardiovascular and Pulmonary diseases (T32)

2008-2010 American Heart Association predoctoral fellowship

April $2008 \quad$ Van Liere convocation day poster award winner

April $2009 \quad$ Van Liere convocation day poster award winner

April $2009 \quad$ Van Liere convocation day oral presentation award winner

$2010 \quad$ Cardiovascular section award winner (APS)

May $2010 \quad$ Outstanding doctoral student award

\section{PROFESSIONAL SOCIETIES}

2006-present American College of Sports Medicine

2008-present American Physiological Society

2008-present AAAS

\section{PRESENTATIONS}

Poster:

Dabkowski E.R, Williamson C.L, Hollander J.M. Increased susceptibility of mitochondrial subpopulations to diabetic cardiomyopathy associated dysfunction. FASEB J. 2007 21:732.25. 
Dabkowski R. Erinne, Williamson, L. Courtney, Hollander, M. John. Increased Susceptibility of mitochondrial subpopulations to diabetic Cardiomyopathy associated dysfunction. Van Liere Research Convocation Day. West Virginia University, 2007.

Dabkowski E.R, Williamson C.L, Hollander J.M. Quantitative proteomic analysis of distinct mitochondrial subpopulations in diabetic myocardium. FASEB J. 2008 22:1226.36.

Dabkowski E.R, Williamson C.L, Hollander J.M. Mitochondria-Specific Transgenic Overexpression of Phospholipid Hydroperoxide Glutathione Peroxidase (GPx4) Attenuates Ischemia/Reperfusion Associated Cardiac Dysfunction. Van Liere Research Convocation Day 2008.

Dabkowski E.R, Williamson C.L, Hollander J.M. Dysfunction in Spatially Distinct Cardiac Mitochondrial Subpopulations during Diabetic Insult. Mitochondria 2008, Bethesda M.D.

Dabkowski E.R, Williamson C.L, Frisbee J.C, Brock R.W, Hollander J.H. Distinct Mitochondrial Subpopulation Response in a Type 2 Diabetic Model. FASEB J. 2009 23:990.22.

Dabkowski E.R, Williamson C.L, Baseler W.A, Hollander J.M. . Distinct Mitochondrial Subpopulation Response in a Type 2 Diabetic Model. Van Liere Research Convocation Day 2009.

Dabkowski E.R, Williamson C.L, Baseler W.A, Hollander J.M. Dysfunction In Spatially Distinct Cardiac Mitochondrial Subpopulations During Type 1 diabetes and Protection with Overexpression of Mitochondrial specific Phospholipid Hydroperoxide Glutathione Peroxidase (GPX4). Van Liere Research Convocation Day 2010. 
Dabkowski E.R, Croston T.L, Baseler W.A, Williamson C.W, Hollander J.M. Mitochondrial Overexpression of Phospholipid Hydroperoxide Glutathione Peroxidase 4 (mPHGPx) Provides Cardioprotection From Type 1 Diabetes Mellitus Insult. FASEB J. $2010201024: 789.2$.

\section{Oral Presentations:}

Dabkowski E.R, Williamson C.L, Baseler W.A, Hollander J.M. Dysfunction In Spatially Distinct Cardiac Mitochondrial Subpopulations During Type 1 diabetes and Protection with Overexpression of Mitochondrial specific Phospholipid Hydroperoxide Glutathione Peroxidase (GPX4). Van Liere Research Convocation Day 2009.

Dabkowski E.R, Croston T.L, Baseler W.A, Williamson C.W, Hollander J.M. Mitochondrial Overexpression of Phospholipid Hydroperoxide Glutathione Peroxidase 4 (mPHGPx) Provides Cardioprotection From Type 1 Diabetes Mellitus Insult. FASEB J. 2010. Translational Research in Metabolic Syndrome and Cardiovascular Disease: Swine versus Mouse Models.

\section{PUBLICATIONS}

\section{Articles:}

Williamson CL, Dabkowski E.R, Dillman WH, Hollander JM. Mitochondria protection from hypoxia/reoxygenation injury with mitochondrial heat shock protein 70 (mthsp70) overexpression. Amer. J. Physiol.294:H249-H256, 2008.

Dabkowski E.R, Williamson C.L, Hollander J.M. Mitochondria-Specific Transgenic Overexpression of Phospholipid Hydroperoxide Glutathione Peroxidase (GPx4) Attenuates Ischemia/Reperfusion Associated Cardiac Dysfunction. Free Radical Biol Med. 2008 Sep 15; 45(6):855-65.

Dabkowski E.R, Williamson CL, Bukowski VC, Chapman RS, Leonard SS, Peer CJ, Callery PS, Hollander JM. Diabetic cardiomyopathy-associated dysfunction in spatially 
distinct mitochondrial subpopulations. Am J Physiol Heart Circ Physiol. 2009 Feb; 296(2):H359-69.

Dabkowski E.R, Baseler W.A, Williamson C.L , Powell M., Hollander J.H. Mitochondrial Dysfunction in the Type 2 Diabetic Heart is Associated with Alterations in SpatiallyDistinct Mitochondrial Proteomes. Am J Physiol Heart Circ Physiol. 2010 Jun 11.

Dabkowski E.R, Baseler W.A, Williamson C.L, Hollander J.M. Proteomic Alterations of Distinct Mitochondrial Subpopulations in the Type 1 Diabetic Heart: Contribution of Protein Import Dysfunction. (Submitted AJP)

Dabkowski E.R, Baseler W.A, Williamson C.L, Hollander J.M. Mitochondrial Overexpression of Phospholipid Hydroperoxide Glutathione Peroxidase 4 (mPHGPx) Provides Cardioprotection From Type 1 Diabetes Mellitus Insult. (In Preparation).

Williamson C.W, Dabkowski E.R, Baseler W.A, Croston T.L, Always S.W, Hollander J.M. Enhanced Apoptotic Propensity in Diabetic Cardiac Mitochondria: Influence of Subcellular Spatial Location. Am J Physiol Heart Circ Physiol. 2009 Dec 4.

Ryan M.J, Jackson J.R, Hao Y, Williamson C.L, Dabkowski E.R, Hollander J.M, Always S.E. Suppression of Oxidative Stress by Resveratrol After Isometric Contractions in Gastrocnemius Muscles of Aged Mice. J Gerontol A Biol Sci Med Sci.2010 May 29. (Epub ahead of print).

\section{$\underline{\text { Abstracts }}$}

Horn, Renate, Institut für Molekulare Physiologie und Biotechnologie; Georgi, Laura, Clemson University; King, James, Clemson University; Dabkowski,Erinne, Clemson University; Abbott, Albert G, Clemson University. RNAi approach for characterization of the anthocyanin pathway in peach. 
Dabkowski E.R, Williamson C.L, Hollander J.M. Increased Susceptibility of mitochondrial subpopulations to diabetic Cardiomyopathy associated dysfunction. FASEB J. 21(6):A819, 2007

Williamson C.L, Dabkowski E.R, Hollander J.M. Contractile Dysfunction in the diabetic heart is associated with enhanced apoptosis and decreased Hsp25 phosphorylation. FASEB J. 21 (6):A1342, 2007.

Dabkowski E.R, Williamson C.L, Hollander J.M. Increased Susceptibility of mitochondrial subpopulations to diabetic Cardiomyopathy associated dysfunction. Van Liere Research Convocation Day, West Virginia University, 2006.

Dabkowski E.R, Hollander JM, Powell M, Razunguzwa T, Perrotta PL. Biomarker discovery strategy for mitochondrial dysfunction using iTRAQ and a high throughput maldi TOF/TOF mass spectrometer. Building Biomedical Research in West Virginia. CoBRE/INBRE, Charleston, WV, November 2007.

Dabkowski E.R, Williamson C.L, Hollander J.M. Quantitative proteomic analysis of distinct mitochondrial subpopulations in diabetic myocardium. FASEB J. 2008 22:1226.36.

Williamson C.L, Dabkowski E.R, Hollander J.M. Enhanced apoptotic propensity in diabetic cardiac interfibrillar mitochondria. FASEB J. 2008 22:1238.19.

Dabkowski E.R, Williamson C.L, Hollander J.M. Mitochondria-Specific Transgenic Overexpression of Phospholipid Hydroperoxide Glutathione Peroxidase (GPx4) Attenuates Ischemia/Reperfusion Associated Cardiac Dysfunction. Van Liere Research Convocation Day 2008. 
Dabkowski E.R, Williamson C.L, Hollander J.M. Dysfunction In Spatially Distinct Cardiac Mitochondrial Subpopulations During Diabetic Insult. MITOCHONDRA 2008, Bethesda M.D.

Dabkowski E.R, Williamson C.L, Frisbee J.C, Brock R.W, Hollander J.H. Distinct Mitochondrial Subpopulation Response in a Type 2 Diabetic Model. FASEB J. 2009 23:990.22.

Williamson C.L, Dabkowski E.R, Brock R.W, Frisbee J.C, and Hollander J.M. Protection from Diabetes-Associated Apoptosis with Heat Shock Protein 27 Overexpression. FASEB J. 2009 23:953.18.

Baseler W.A., Dabkowski E.R, Williamson C.L, and Hollander, J.M. Skeletal Muscle Mitochondrial Subpopulation Response in a Type 2 Diabetic Mouse Model. FASEB J. 2009 23:LB93.

Vejandla H.L, Kothur A., Dabkowski E.R, Hollander J.M, Frisbee J.C, and Brock R.W. C-peptide confers protection in renal cortical endothelial cells during Type I diabetes by preventing the phosphorylation of glucose-6-phosphate dehydrogenase. FASEB J. 2009 23:971.12

Vejandla H.L, Frisbee J.C, Dabkowski E.R, Hollander J.M, Kothur A., and Brock R.W. Hyperglycemia-induced mitochondrial dysfunction and oxidant generation in mouse renal microvascular endothelial cells is reversed by C-peptide. FASEB J. 2009 23:594.15.

James M.L, Dabkowski E.R, Hollander J.M, Ripke I., Baumann A., Kothur A., Wunder C., and Brock R.W. Carbon monoxide provides antioxidant protection in hepatic sinusoids during a remote inflammatory stress by reducing carbonylated MnSOD . FASEB J. 2009 23:982.3 
Dabkowski E.R, Williamson C.L, Baseler W.A, Hollander J.M. Dysfunction In Spatially Distinct Cardiac Mitochondrial Subpopulations During Type 1 diabetes and Protection with Overexpression of Mitochondrial specific Phospholipid Hydroperoxide Glutathione Peroxidase (GPx4). Van Liere Research Convocation Day 2010.

Dabkowski E.R, Croston T.L, Baseler W.A, Williamson C.W, Hollander J.M. Mitochondrial Overexpression of Phospholipid Hydroperoxide Glutathione Peroxidase 4 (mPHGPx) Provides Cardioprotection From Type 1 Diabetes Mellitus Insult. FASEB J. $2010201024: 789.2$.

Baseler W.A, Williamson C.L, Dabkowski E.R, Croston T.L, Hollander J.M. Mitochondria-specific overexpression of phospholipid hydroperoxide glutathione peroxidase (GPX4) attenuates ischemia/reperfusion (I/R) associated apoptosis. FASEB J. $201024: \operatorname{lb560.}$

Croston T.L, Dabkowski E.R, Williamson C.L, Baseler W.A, Hollander J.M. Mitochondrial subpopulation-specific proteomic alterations in the type 2 diabetic heart. FASEB J. 2010 24:Ib573

Vejandla H.L, Dabkowski E.R, Hollander J.M, Brock R.W. C-peptide restores mitochondrial complex 1 activity and lowers superoxide levels in high glucose-treated murine renal microvascular endothelial cells. FASEB J. 2010 24:571.10.

Stricker J.C, Nichols C.E, Katz A, Fancher I, Cuppett T.C, Miletti K, Vasisko C, Dabkowski E.R, Baseler W.A, Hollander J.M, Morissette M.R. Characterization of regression of exercise-induced cardiac hypertrophy. FASEB J. 2010 24:Ib593. 


\section{RESEARCH GRANTS}

\section{$\underline{\text { Received: }}$}

American Heart Association Predoctoral Fellowship

American Heart Association

"Influence of a Diabetic Phenotype on Specific Mitochondrial Subpopulations in the Heart"

Role: PI

Score: 1.4

Status: July 1, 2008-June 30, 2010

The goal of these studies is to determine how different mitochondrial subpopulations are affected with diabetic cardiomyopathy. 